CIHM

Microfiche

Series

(Monographs)
ICMH

Collection de microfiches (monographies)

Canadian Institute for Historical Microreproductions / Institut canadien de microreproductions historiques
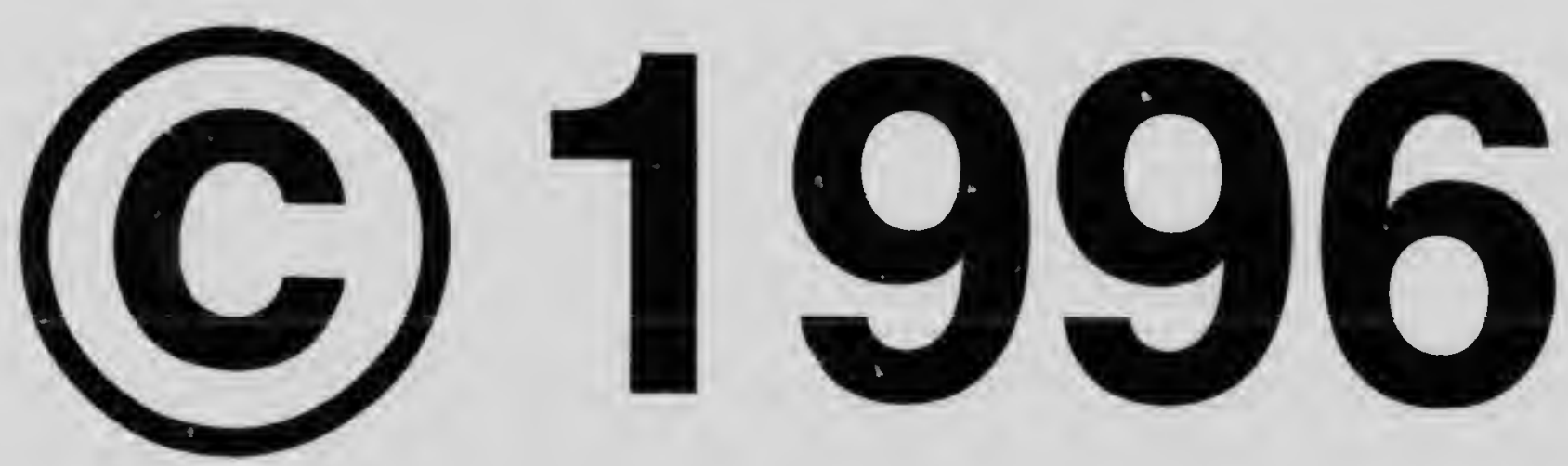


\section{Technical and Bibliographic Notes / Notes techniques et bibliographiques}

The Instltute has attempted to obtain the best orlginal copy available for filming. Features of thls copy which may be blbliographlcally unique, which may alter any of the Images in the reproduction, or whlch may signiflcantly change the usual method of filming are checked below.

\section{Coloured covers /}

Couverture de couleur

\section{Covers damaged/}

Couverture endommagée

\section{Covers restored and/or laminated /}

Couverture restaurée etlou pelliculée

Cover title nissing / Le titre de couverture manque

Coloured maps / Cartes géographiques en couleur

Coloured ink (i.e. other than blue or black) /

Encre de couleur (i.e. autre que bleue ou noire)

Coloured plates and/or illustrations /

Planches etou illustrations en couleur

Bound with other material /

Relié avec d'autres documents

Only edition available /

Seule édition disponible

Tight binding may cause shadows or distortion along interior margin / La reliure serrée peut causer de l'ombre ou de la distorsion le long de la marge intérieure.

Blank leaves added during re storations may appear within the text. Whenever possible, these have been omitted from filming / II se peut que certaines pages blanches ajoutées lors d'une restauration apparaissent dans le texte, mais, lorsque cela était possible, ces pages n'ont pas été filmées.

Additional comments /

Commentaires supplémentaires:
L'Instltut a microfilmé le mellleur exemplaire qu'il lul a été possible de se procurer. Les détails de cet exemplalre qul sont peut-etre unlques du point de vue bibllographlque, qul peuvent modifier une Image reproduite, ou qul peuvent exlger une modification dans la méthode normale de filmage sont indiqués ci-dessous.

Coloured pages / Pages de couleur

Pages damaged / Pages endommagées

Pages restored and/or laminated /

Pages restaurées etlou pelliculées

Pages discoloured, stained or foxed /

Pages décolorées, tachetées ou plquées

Pages detached / Pages détachées

\section{Showthrough i Transparence}

Quality of print varies /

Qualité inégale de l'impression

Includes supplementary material /

Comprend du matériel supplémentaire

Pages wholly or partially obscured by errata slips, tissues, etc., have been refilmed to ensure the best possible image / Les pages totalement ou partiellement obscurcies par un feuillet d'errata, une pelure, etc., ont été filmées à nouveau de façon à obtenir la meilleure image possible.

Opposing pages with varying colouration or discolourations are filmed twice to ensure the best possible image / Les pages s'opposant ayant des colorations variables ou des décolorations sont filmées deux fois afin d’obtenir la meilleure image possible.

This hem is fllmed at the reduction ratio checked below I

Ce document eat films au taux de ríduction indiqus ci-deseous.

\begin{tabular}{|c|c|c|c|c|c|}
\hline $10 x$ & $14 x$ & $18 x$ & $22 x$ & $26 x$ & $30 x$ \\
\hline & & & $\checkmark$ & & \\
\hline
\end{tabular}


The copy fllmed here has been reproduced thenks to the generesity of:

National Library of Canada

The images appearing here are the best quality possible considering the conditlon and iegibility of the orlginal copy and In keeplng with the filming contract speciflcations.

Original coples in printed paper covere are filmod beglnning with the front cover and onding on the last page with a printed or Illustrated impres. sion. of the back cover when appropriate. All other original copies are filmed beginning on the flrat page with a printed or lllustrz ted Impres. sion. and ending on the last page with a printed or illustrated impression.

The last recorded frame on each microflche shall contain the symbol $\rightarrow$ Imeaning "CON. TINUED"I, or the symbol $\nabla$ (meaning "END"). whichever applies.

Maps, plates, charts, otc., may be filmed at different reduction ratios. Those 800 large 80 be entirely included in one exposure are filmed beginning in the upper lett hand corner, loft to right and top to bottom, as many frames as required. The following diagrams lllustrate the mothod:
L'exemplaire fiimb fut raproduit gráce la odndrosite de:

\section{Bibliothèque nationale du Canada}

Les imeges suiventas ont det reproduites avec le plus grand soin. compta tanu da la condition ot de la nottet de l'exemplaire filmb. at an conformlto avec les conditions du contrat de fllmage.

Les exemplaires originaux dont la couvertura an papier est Imprlmbe sont fllmbs en commencant par le premier plat ot en terminant soit par la dernibre page qul comporte une emprainta d'Impression ou d'illustration. soit par la second plat, selon le cas. Tous los autres examplaires originaux sont filmbs en commonçant par la premidre page qui comporte une ampreinte d'impression ou d'lllustration et en serminant par ia dernibre peoe qui comporte una talle emprainte.

Un des symboles suivents apparaitra sup la dernidre image de chaque microfiche. selon le cas: le symbole $\longrightarrow$ signifie "A SUIVRE". le symbole $\nabla$ signifie "FIN".

Les cartes. planches. tobleaux. etc.. peuvent être filmbs des taux de reduction differents. Lorsque le document est trop grand pour être reproduit en un soul clichb. il ast filmo partir de l'angle supbriour gauche, de gauche droite. ot de haut en bas. on prenant le nombre d'Images nocessaire. Les diagrammes suivants lllustront la mothode.
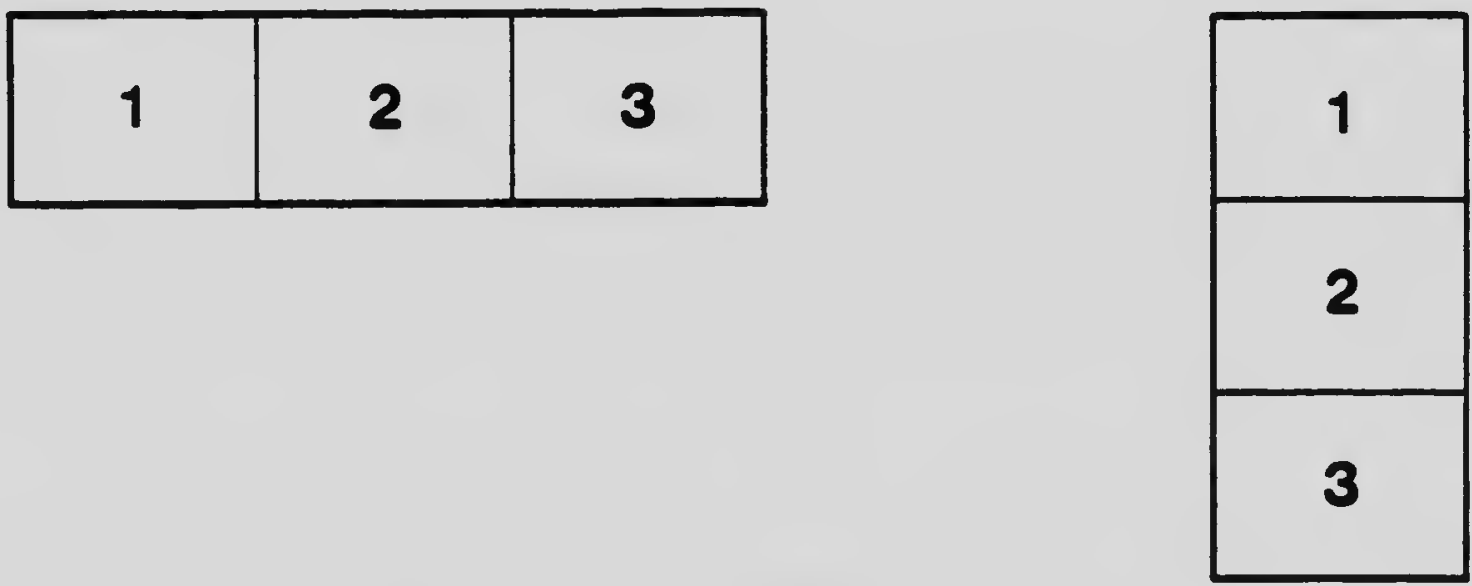

\begin{tabular}{|l|l|l|}
\hline 1 & 2 & 3 \\
\hline 4 & 5 & 6 \\
\hline
\end{tabular}




\section{MICROCOPY RESOLUTION TEST CHART}

(ANSI and ISO TEST CHART No. 2)
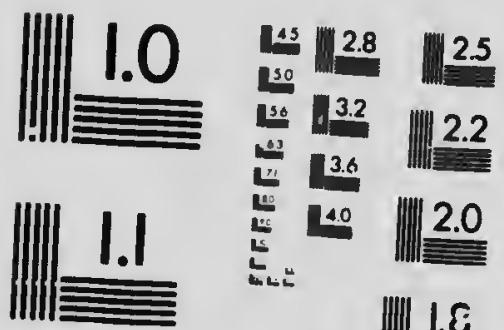

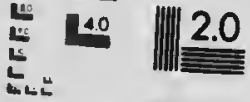
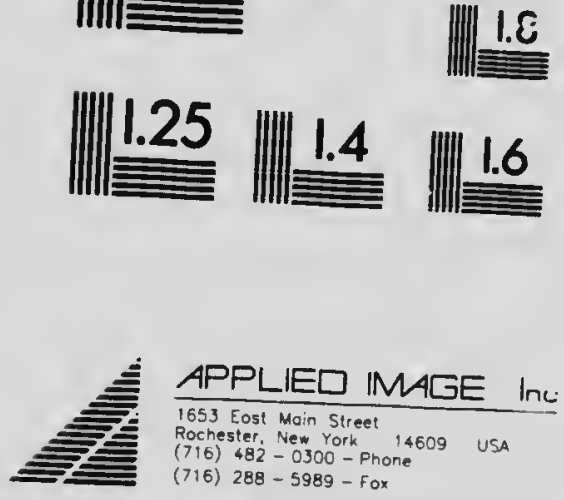
$\because$

,

. 


\section{LA \\ RUCHE CANADIENNE \\ CULTURE DES ABEILLES \\ - PAR -}

\section{ALEC SANTERRE}

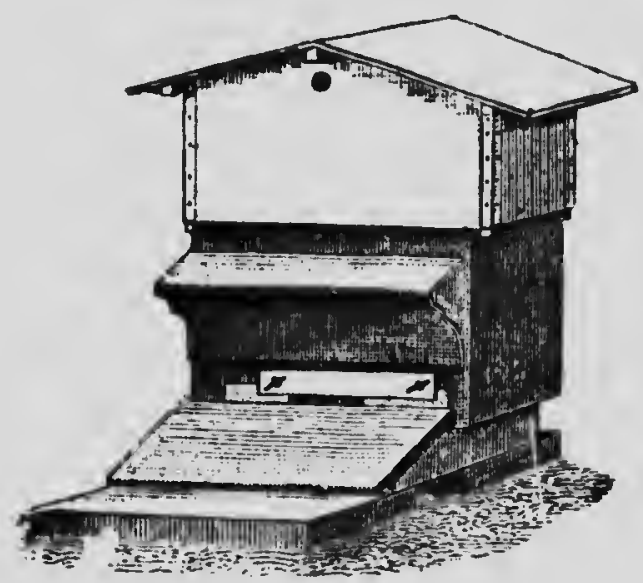

QUEBEC

Iup. Darveau, Jos. Beauchamp Prop.

82, Cote de la Montagne 


\section{* LA *}

\section{RUCHE CANADIENNE'}

\section{CULTURE DES ABEILLES \\ - PAR -}

\section{ALEC SANTERRE}

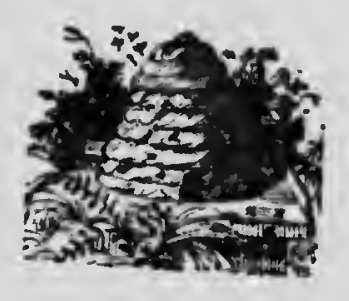

QUÉBEC

Imp. Darveau, Jos. Beauchamp Phop.

82, Cóte de la Mon tagne

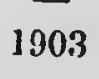

|4I 


\section{$5+527$}

S.R.i

Juroginthi conforménent it l'dete du l'arleasent du Canada, on

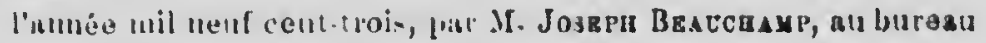
lu Ministre dir l’. Igrienllute, Ötawa. 


\section{INTRODUCTION.}

Linpiculture est me des lirninlies de l'ugrieulture que l'ou néglige trop duns notre puy's.

On n pm. pendant longtemps, redonter les rigueurs du climat pour s'udonner is la culture des abeilles, mnis l'objection ne snurnit tenir najourd hui pue des expériences ort été fnites non senlement daus les parties les plus temperies du Canndn, monis duns les regions uin les rignenrs de l'lliver se font le plus lc.ugtemps sentir. Pour suvous mointennut, que dans In partie de ln province de Quélece qui est expluitée, il n'est

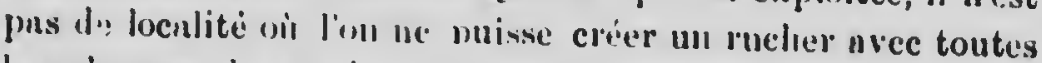

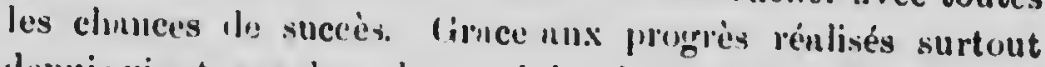

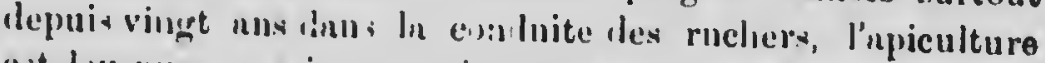
est devenne moe scienee, mnis cette sejence ne sumbit eftruyer personne, car elle noffle yne des difticultés relatives pour les personnes intelligentex qui vinlent finire preure de quelque bonne volunté.

Le micl, sirop maturel, ilemi fluide et de snveur sucré, qu'élaborent les nhuilles et qu'elles dépusent duns les alvéoles de leurs gnatenux le cire, est un prorluit à In fois très nutritif, très snin ei qui jouit d'une grande fuveur dans toutes les funilles 'jui penvent s'en procurer. Il est aussi très économique, car il fuit bunucoup plus de protit que n'importe quelles confitures. Il y a donc lì une source de richesse qui reste ignorée.

Les habitunts des campugnes peuvent puiser cetto source, sans se détourner de leurs autres occupations agricoles, car la culture les abeilles ne demande que peu do trarail, et líxigr an début quiun frible capital. 
Si l'apieultmo premait tunte l'extonsion qu'elle pent avoir, les cultivateins, et, far suite, les habitants des villes, les ourriers des fabriques, auraient in len portée une subs. tance alinentaire saine, naturelle lout ils pouracut toujours vériticer l'urignims.

Ancune secupation lumles next mieux is la portéc de tous que ln culture los abeilles et ne demunde un copital plus inoliqque. II n'est pas bzsoin de benucoup de terrain, l'espnce nécessaire pour plucer quelyuos ruches suftit, et si les ubeilles du pauvre vout duus les clamps du voisin riche seupnrer du nectar de ses Heurs, elles lui donuent ume large compensation en fécondunt colles yn'elles visiteut.

Dans les puys oì l'npiculture est plus déréloppée, le micl s'écoule tr'es fincilement et il n'est guère de produits de la ferıne, s'il en est même, qui ı’npportent le protits plus nets; car l'expérience nous démontre, qu'en proportion du remlement, les Jéloursés et ln muin-d'mure sont nhsolument insiguifinnts.

Fring Le but le cet ourrnge est de mienx fnire comnnitre l'apiculture, ses ressources, ses avintuges tout aussi bien que la conduite du rucher. Il uous a fullu nous tenir duns un calle restreint pour ne pas fatiguer le lecteur et l'apprentiapiculteur, par ume séric le létuils très intéressants sur les abeilles, mnis qui nunient pu purnitre mu pou fontidieux.

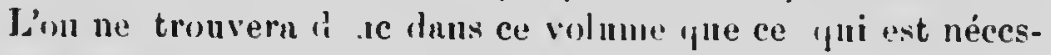
saire à ceux qui voulent s'ulommer à la culture des aheilles

Comme il est hon de profitor les resultatide l'experience, nuus devons ici recommuitre que mons nvous largemcont puisé aux sources les plus nutorisfes ru la mutièe. Linuvruge de Daclant "l'Aheille et In Ruclic", cului de Eıl. Bertrand "Contuite du Rucher" ourruges très complets et qui font autorité, nous ont été l'un sccours précieux tout nussi bien que lcs conversations yuc nous avons enes avec M.M. I. N. Péloquin, re St. Hyrcintle, Verret et le 1)r. (⿳rusverul de 
Charlesbuarg, Luc Dopuis, du village des Aulmaies, yni dejnis de longues années s'alonnent avee succès à l'apienlture at qui sujourdhui aiment l'nutunt plus 'eur travail qu'il leur. rapporte de bons bénétices

l. est bon de mentionner ici pou: prouver les re-ultuts que l'on pent atteinulre dans notre provinee par la enlture

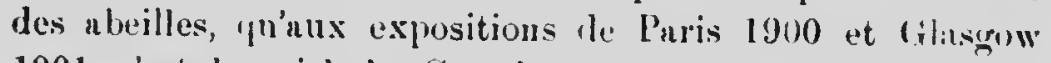
1901, e'est le niel ilu Camala, provenant le ehe\% .11 Jase Dupuis, qui a obtenu les plus hautes récompenses.

Nous pourons lone, mème en dépit de notre eiimit rigoureux, obtenir l'excellents résultats non senlement quant à la quantité, mais anssi quant à la qualité, comme on lo verra quand nous parlerons de la récolte lu miel.

C'est le motif yni nous a finit comlenser dhns mn aussi petit volume qur: possible, ee qu'il est nécessaire de connaitre et si nous pouvons, en le pulliant, reudre quelqnes services à nos compatriotes, nous serons sutisfuits in lint que nons aurons ntteint. 


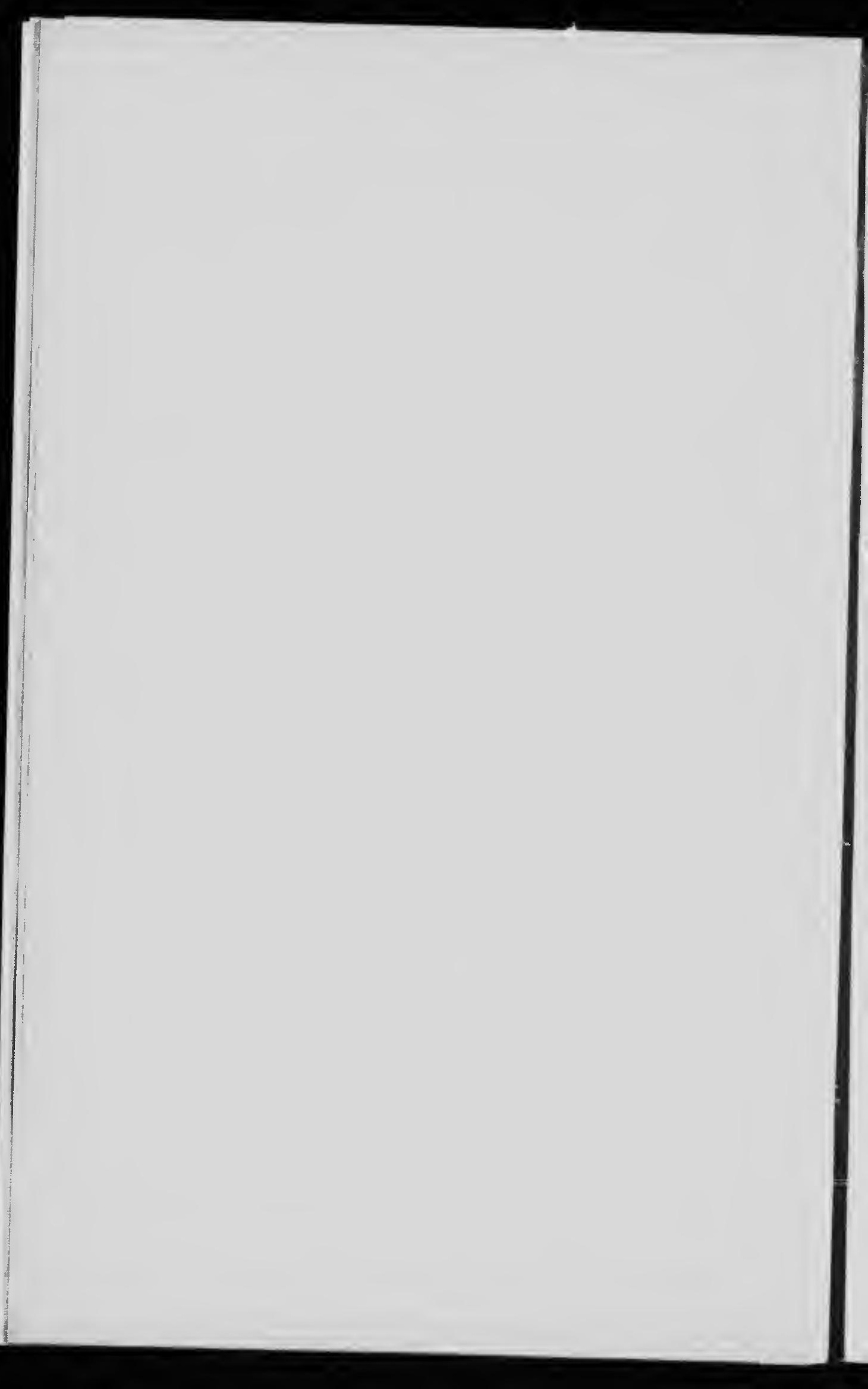




\section{LA RUCHE CANADIENNE}

CULTURE DES ABEILLES

\section{CHAPI'RE PREMIER}

DES ABEILL.E.S.

L'tilité des abeilles pour l'agriculture.

L'upiculture n'intéresie pus seulement l'ngriculteur par sas importants pro.luits, mais elle lui rend nussi bien sonvent service d'une manière indircete.

'Toutes les fois quion cultive les pluntes pour en obtenir soit des graines, soit des fruits et que les plantes sont mellifères, les uleilles en butinunt de Heur en Heur contribuent, pour une part importante, a nuguenter le prodnit de la récolte par la diffusion ilu polien

C"est uinsi que le coltivateur qui u des ruches dans son verger verra s'uccroitre la quantité moyenne de fruits qu'il obtient chaque année, paree (que, grance aux abeilles, ces fruits auront noué en plus grand nombre. C'ent ainsi que le prysan qui cultive les luricots, les lentilles, les pois, les féves ou encore les graines fonrra ères, verra s'accioitre le produit de ses champs, s'ils sont voisins de ruchers.

Qnant aux prétemlues lévastations que feraient les abeilles en attaqunnt les pêcless, prunes ou d'autres fruits sucrés, elles ne correspond.nt qu'à des légats appureuts qui reposent sur des fa.ts mul observés. Onl ne suurait trop lutter contre ce préjugé, mulhcureusement t:ès répandu, que les alseilles sont nuisibles en cette circonstance ; car il est prouvé d'une manière absolue qu'elles sont illcapnbles de 
déchirer l'unveloppe de ces fruits ; les nbeilles n'en récoltent le sucre que lorspue les fruits ont été nttayués par les oiseanx, les gonples on les frelons, c'est-in-dire loryute les fruits sont dejjì entrunes.

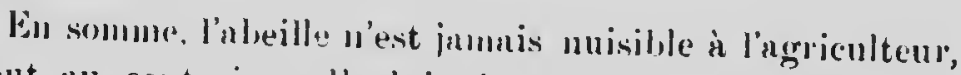
et tout nu contrnire, elle lui vient souvent en nide l'une manière efficaco en nugnucntant la récolte de benucoup de cultures.

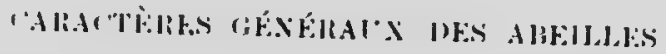

Les abilles sont des insectes nplurtemant in l'ordre des hyméuoptères, ainsi disignés purce qu'ils ont quatre ailes nembrnneuses. Jes nbeilles ne peuvent virre qu'en grnn les agglomérations, une seule nheille étant nussi faible qu'un enfunt qui riunt de naitre, puisqu'elle est puralysée par la fruichenr d'une simple muit al'été.

Ine fumille on colonie d'alcilles se conplose : diue reine on mier femelle fécomle dout ln somle fouction est de pondre des axufs dams les cellules; de milliers d'ulseilles ouvrières on femelles incompletes, qu' s'uccupent in faire tout le truvil

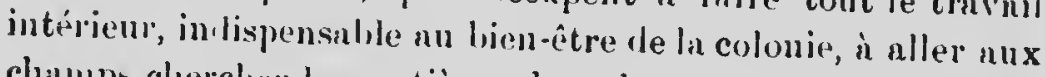
champon clsercher les mntières alout ln pupulation se nourrit, l'enu dont elle n bessin et ln résine on propolis dont elle encluit les fentes on crevasses de l'huhitation; entin, ì certaines éporues die liannée, ln population s'augurente al'ume qunutité de miles on fuux houmlons, dont le nombre pent s'elever de quelques donyines à quelques milliers et qui n'ont

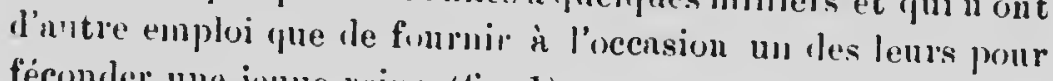
féconder une jeune reine. (fig, 1).

Reine, ouvrières, mâles, couvain - Ce sont les uu. vières ou femelles impropres à la reproduction qui constituent la population l'une ruche ì l'étnt nol'mal, car les mâtes ou faux-bourlons n'apparaissent qu'en nombre restreint nux approches le l'essimugget pen laut les granıles Homisons, rot 


\section{I}
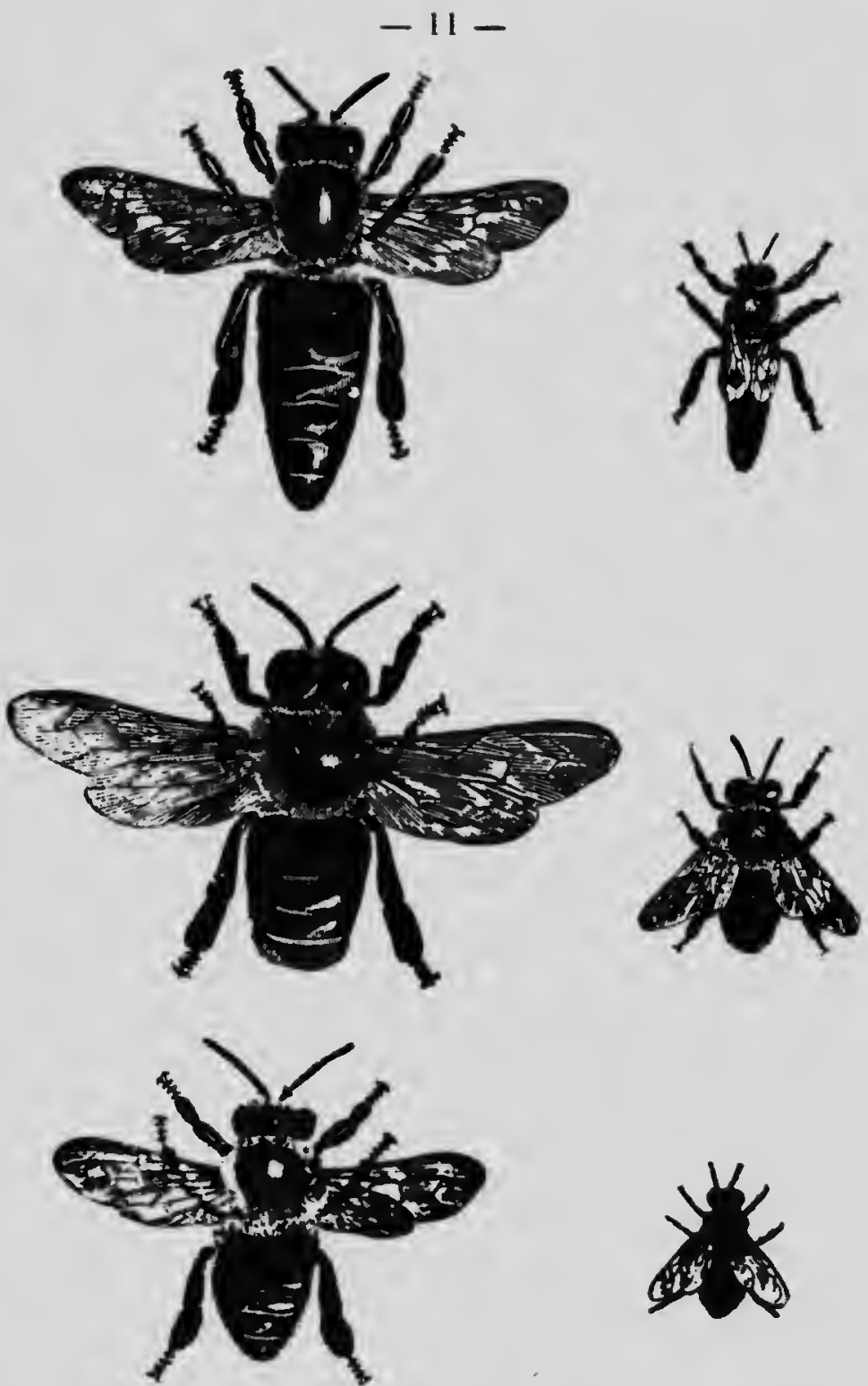

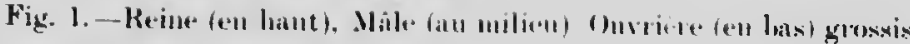
et graulem maturelle. 
il n'existo duss chmpur fumille ou rnchée qu'une senle fomelle

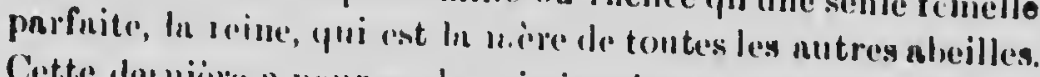

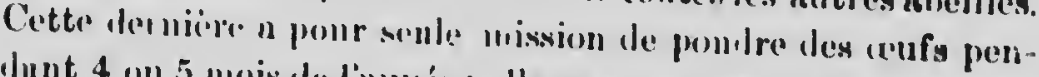

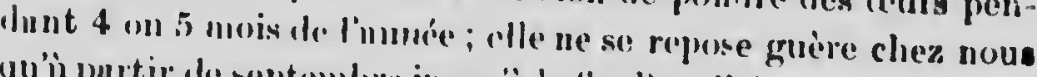

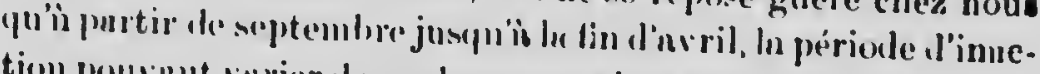

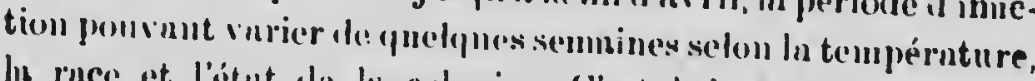
lis rnes et létut de lis colonice. C"est in ln tin du printemps que lu pont. prend sou plus gromel developpenent; alle ne

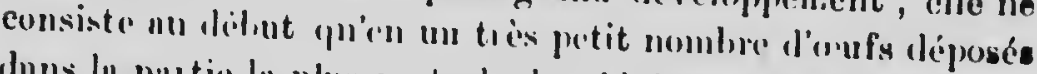

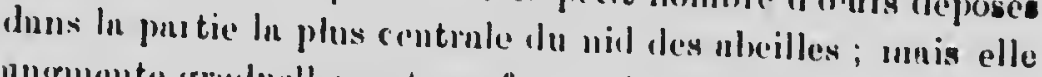

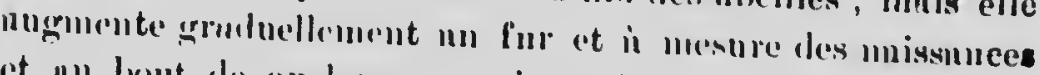

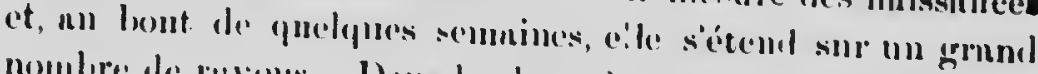

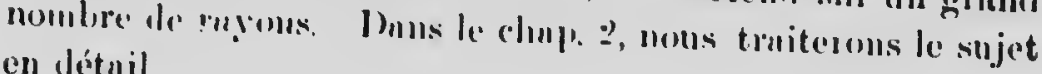
en létuil.

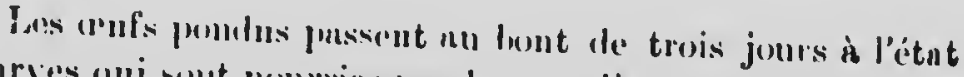

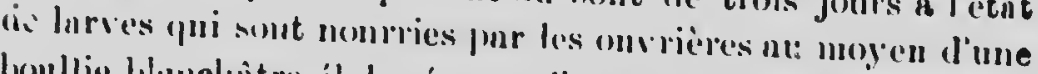

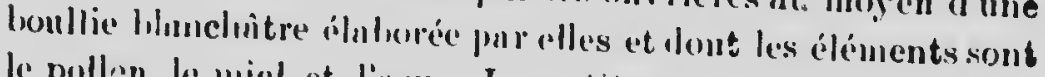
le pollen, le miel et l'eun. Ise petit res linigne dons eette boullic an foml ile la cellule.

Ln luse lenviere regent de la menriture pendunt 5 jonrs envirun, juis elle est enfermée dinns sin collnke nu moyen diun cunverele on operenla, sin transformation en nymphe s'opire et elle solt i li.tat. parfinit 12 jonrs nposes nuvir été emprisommé, soit géncimlement le 2 lme jour npris yue l'uruf a été pondu.

La larve du mile est noumeje penulunt 6. jours et l'éclosion de l'insecte purfait 11 licu environ 24 jours upres la ponte de l'runf. (tig. ?).

La larve de la mére est nourrie pendant is jours. l'emprisonuement duıs ln cellule dure environ $7 !$ jours et l'éclosion a lien le l biue jour environ après que l'reuf n été pondu.

Nous mations ci.contre une table comparative et approximative du temps que les trois genres d'abeilles qui sont 


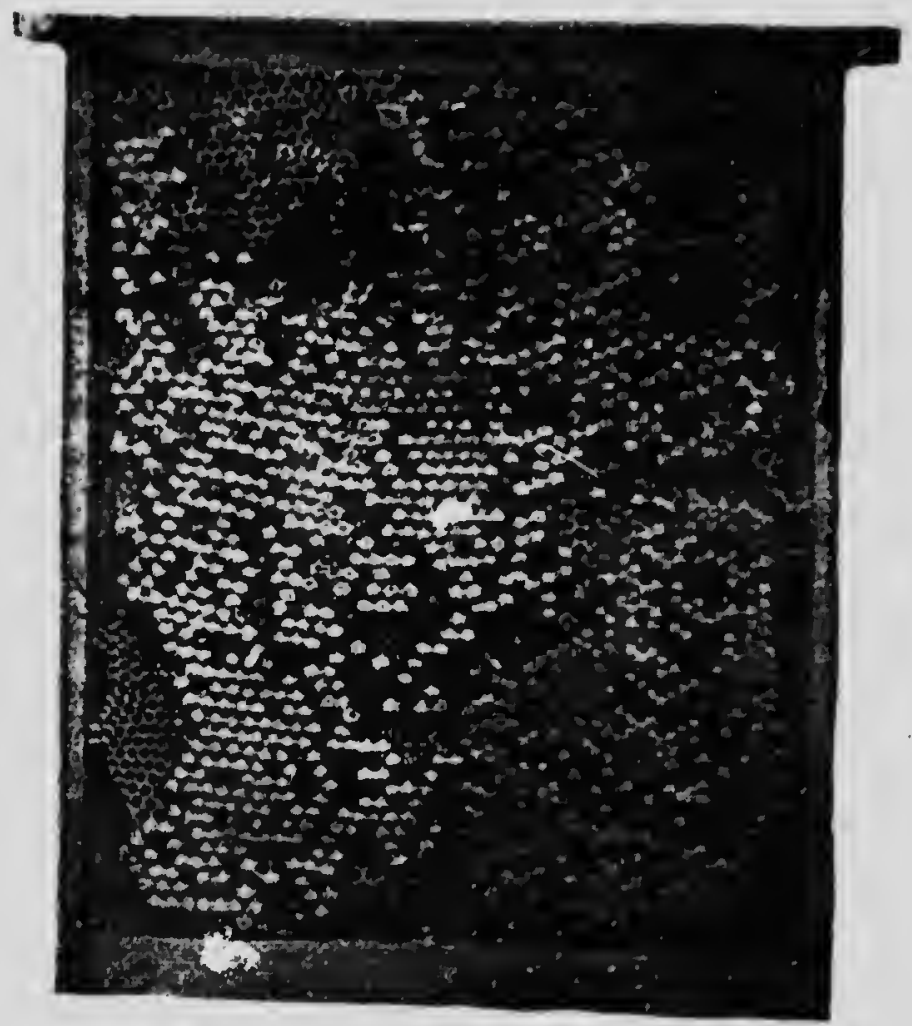

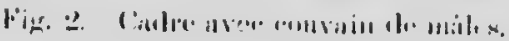

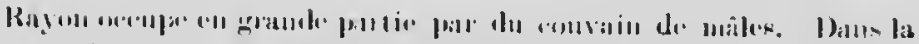

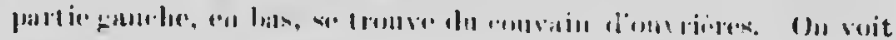

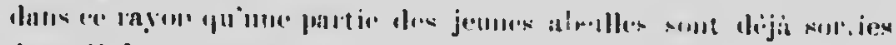

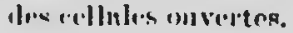


élcrés lans les ruclies mettent ì se développer, table qune nous cimpruntons au live du doetenr A. Dubini, l'A pre.

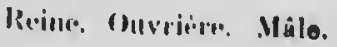

(kuf

\begin{tabular}{cccc} 
jours & 3 & 3 & 3 \\
" & 5 & 6 & $6 \frac{1}{2}$ \\
$“$ & 1 & 2 & $1 \frac{1}{2}$ \\
$"$. & 2 & $\vdots$ & 3 \\
" & 1 & 1 & 1 \\
1 & 3 & 7 & 9 \\
\hline
\end{tabular}

la sortic de la eellule.........

" $1.5 \quad 21$

Il n'y a que deux espèces d'untfs: les arufs mâles, qui no sont pus f'écoulé; et les coufs femelles, qui le sont et produisent soit les ourrières, suit des mères. ("est en dommant pendunt les derniers jours une nourriture plus élaborée is des larves femeiles et en lenr eunstruismat des eellules plus gromles ot ilirigies de lant en las que les onvrières élèvent de nonvelles mères quandle besoin s'en fait sentir, e'e:t-à-lire

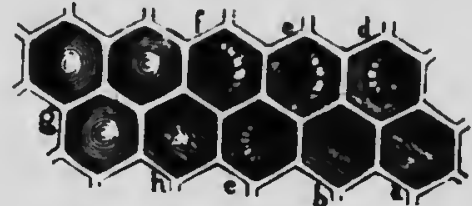

F"ig. 3. Couvain a difféments. itits.

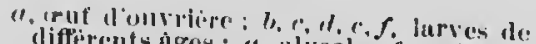
differents nges; $u_{0}$ alieoles fermes pir III couvercle bonibe. contenant du cour. tran a ctat de ( rminsformation: $h$, larre trankformee en abeille prete a sortlr de
l'alveole. sissent générulement des in certain nombre d'henres pour le sorte qu'en cas de suppression d'unc reine dans une ruche l'éclosion des nouvelles peut commencer dès le $10 \mathrm{me}$ ou le 11 me jours après in suppression de l'ancienne, chose importante à noter. (fig. 3 ).

quand la population est trop is l'étroit dans sn demeure (essuimnge) bu quamil la mère est llefee. tueuse on morte. Jorsque les ourrières se fout lo nouvelles mères ponj rem. placer l'uncieune et non pour essaimer, elles choi- 
Lénsemble des coufs, des larves et ilos nymphes sinppello couvain Les opercules des nyomphes, finits d'un mélunge de cire et de pollen, sont poreux; ceux des ourrieres sont plats, ceux des mâles bombós; lca cellules iles meres ont l'appurence de glan's dont l'extrénité est dirigée en lins

\section{I.ES OLVMTHER}

Le's ouvrieres, lit Inlunt, sont les plus petits hubitants

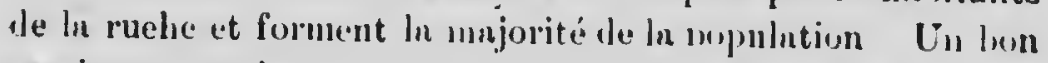
essain ell contient an moins vingt mille et dnus les grondes

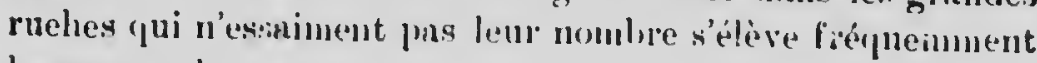
benucoup plus

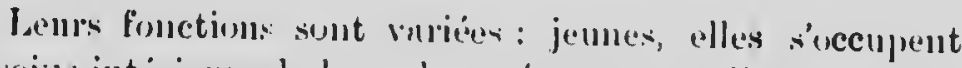

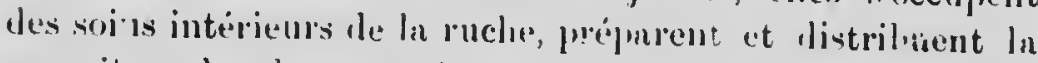
nomriture des lareses, soignent la mere en la larossant et la noumisisnt ; entretiement ln chalene le la ruche, In nettoient do toutes ses immondices ret les callares des abeilles mortes,

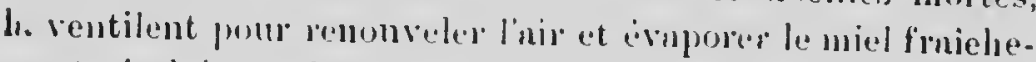
mont resolté, gardent l'entrée contre leurs ennemis on contre d'antres nbeilles qui ponrraient s'y introuluire comme pillareles, fulriphent ln cire ot font les élifices on les réparent au hesoin, ete. Plus vicilles, elles s'ocenpent encore à l'vecasion les mênes solns, mais lent principale leesogne est d'aller í la récolte du miel et lu pollen, qui servent de

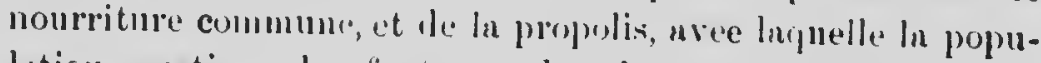
lation inastipue les fentes et boneho les erevinses de son habitntion. Elles vont ausisi chereher de l'mu promr lélayer la nourriture des larves, ete.

Tous les trminux de In rnelee, sauf ln poilte, sont lonc exécutés par les nuvrières. Lus 'pninze premiers jours de leur vie sont eonsilcrés nux soins à domuer un eouvain, à la construction ou in la répmration dexinyons, en un mot mux travaux qui se font dans l'intérieur de ln ruclie. Ce n'est donc que eing semnines environ aprè la ponte d'un aruf d'ouvrière que l'abeille issue de cot muf devient lutineuse : close égralement 
importnute à noter. surtont pour les eontrées courtes

Les nlevilles a une moime moche se comprennent tres bien pour (execoter tont ce qui ist necessuire pour le hien-ritre de

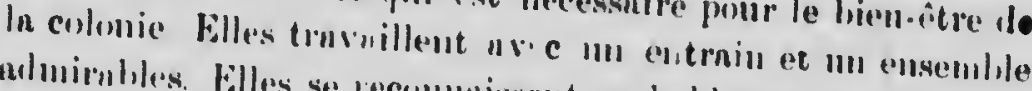
mlmimbles. Filles se recommiscont probullement par l'inlonr.

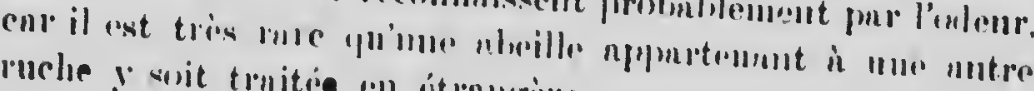

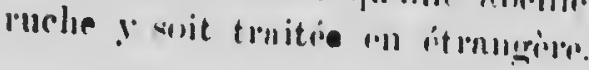

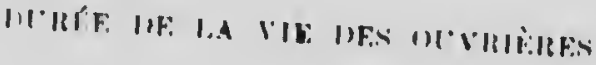

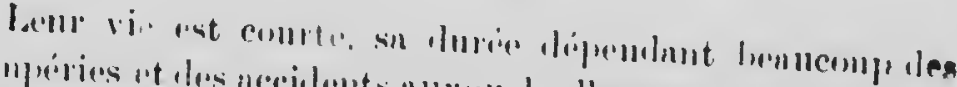

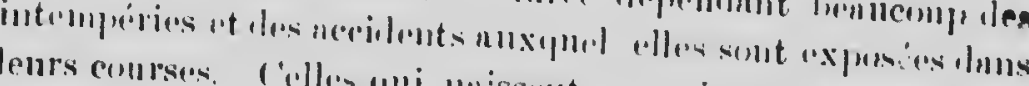

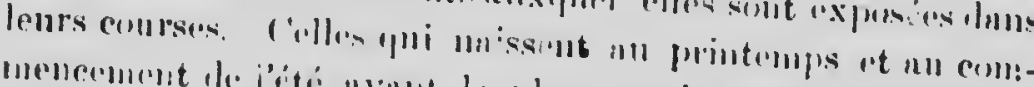

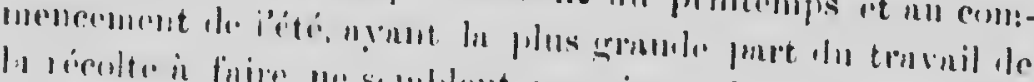

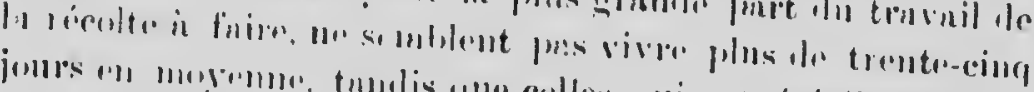

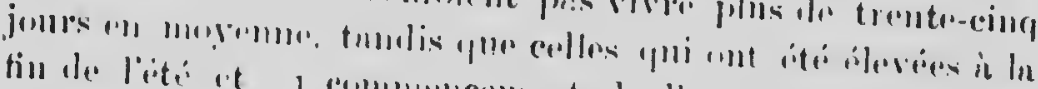

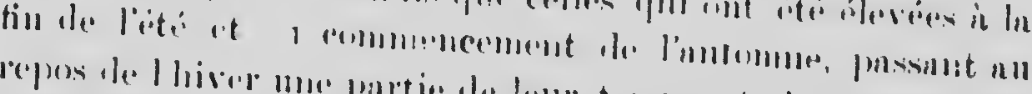

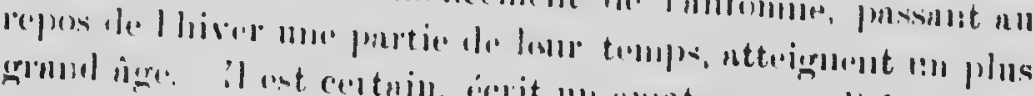

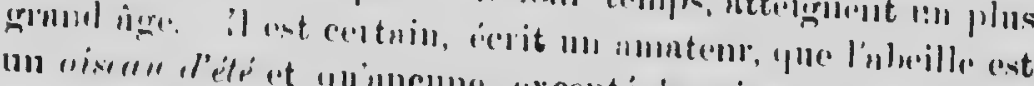

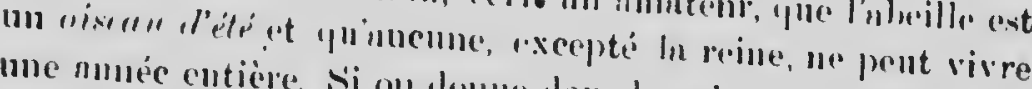

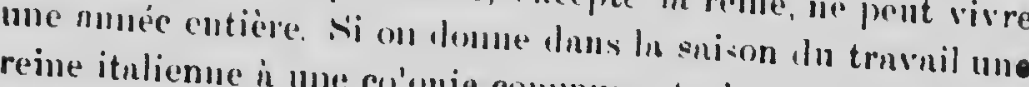
reine itulienue in me co'onic communne, trois mois après il ne restern plux muc seule nheille commume lans la inelie. Or,

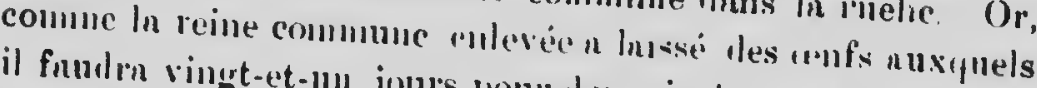

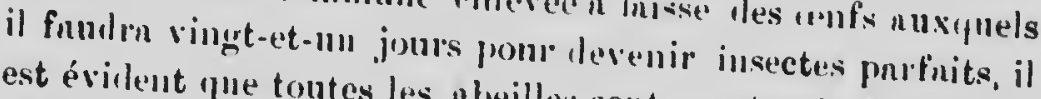
est évident que toutes les aheilles sont mortes lle futigne on d'accilcut dans les 70 jours restunts, ce yui liulue une moyenne de 35 jours duraut lin saison de travil.

Ia perte journaliere d'alieilles l'un essaim logé en ruche vide s'élève, d'uprès notre calcul, is environ trois et quatre pour cent du nombre des alieilles. Si nous supposons que l'essaim possèrluit 35,000 ubeilles quind il a été récolté, comme toutes seront mortes au bont l'environ 70 jours, la moyenne les décès s'élèvera à 500 alıeilles par jour ; mnis, suivant nos 


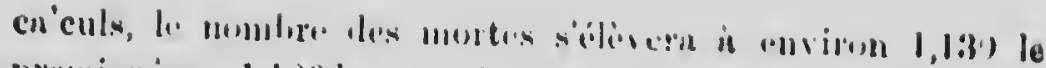

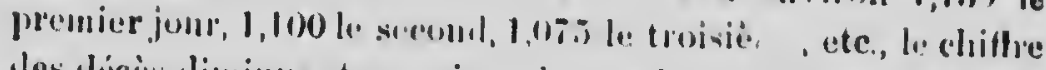

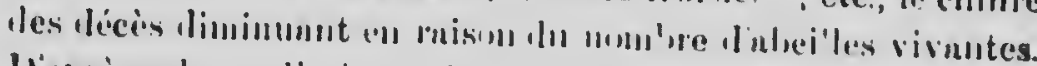

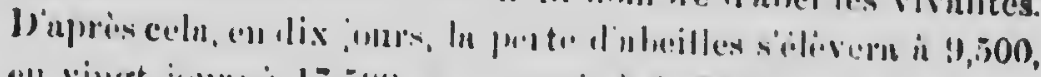

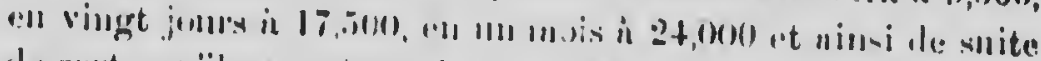

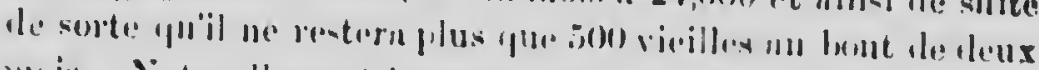

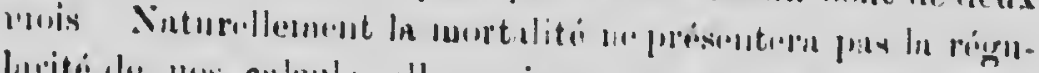

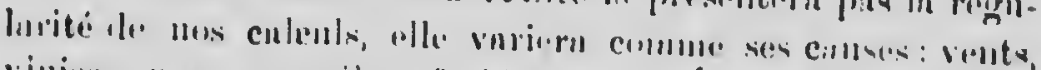

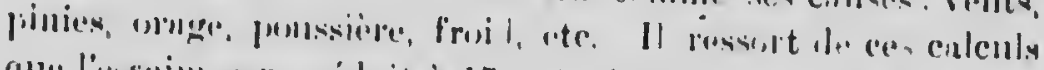

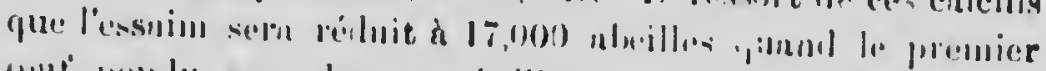

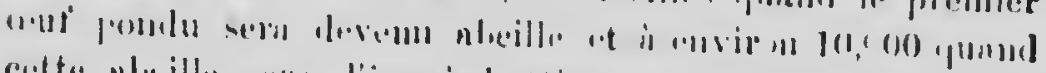

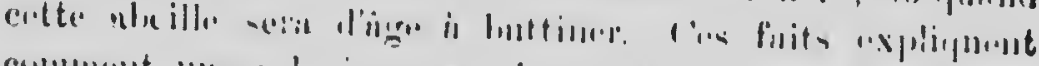

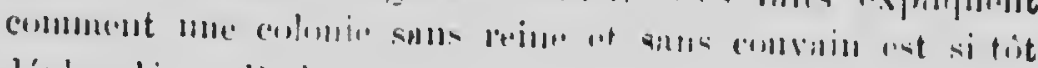
déplenplion. (l'ullut.)

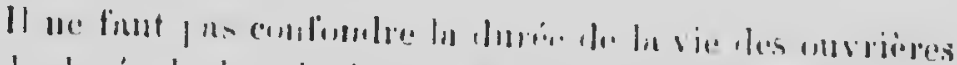

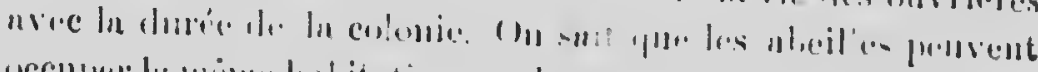

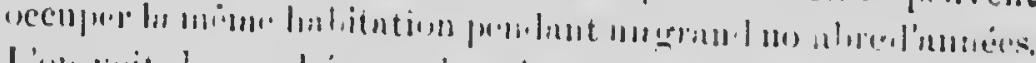

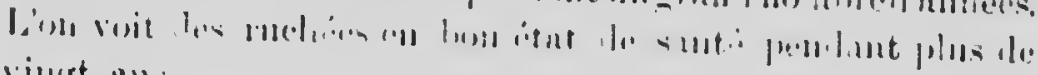
vingt an! :

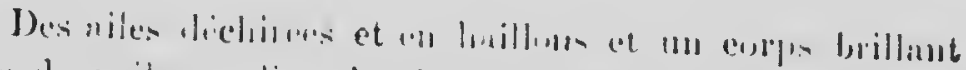

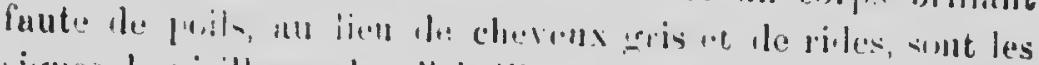

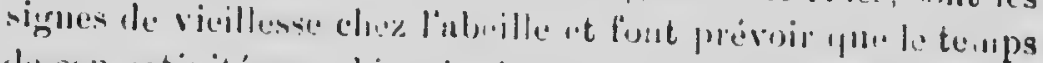

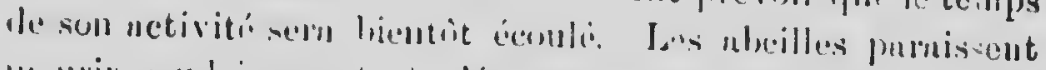

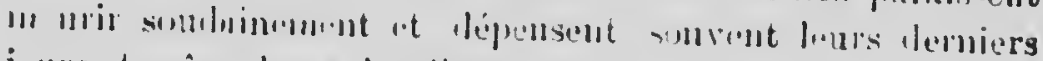

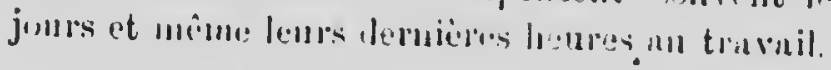

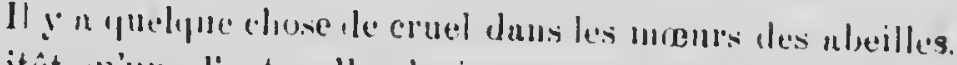

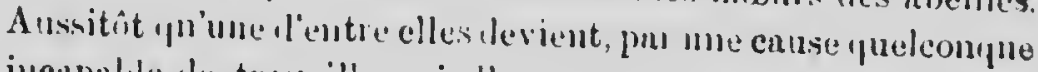
incripnlule de traviller, si clle no meurt pas dans ses efforts pour uller anx chmmpe, ses compagnes In trainent sais pitió hors de lu ruclec, leur auour embrussant toute la fanille et we se divisant pas sur un seal indivilu. Cependur ..' l'ume d'elle appelle a l'aide, des milliess l'antres son $\cdots{ }^{2}$; ì la vengel:

Las $11 \hat{n} ! \cdots$.xistent lans unis rnchio normale_qu'a 


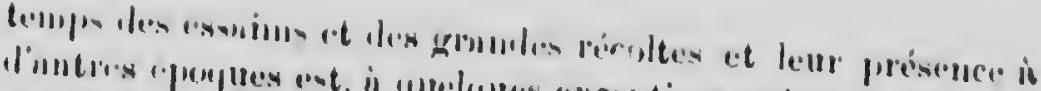

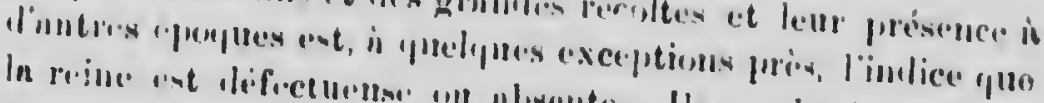

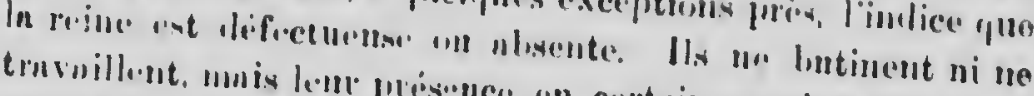

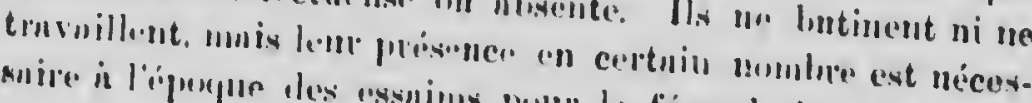

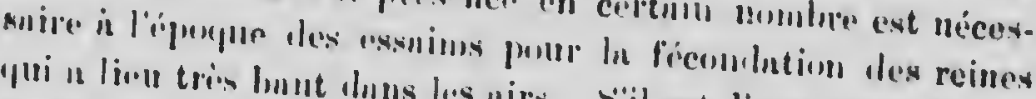

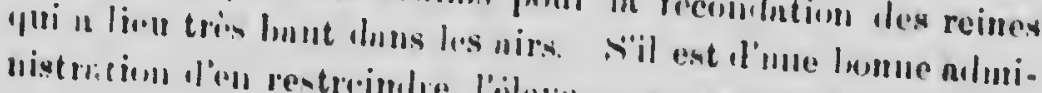

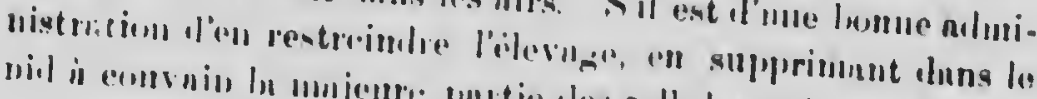

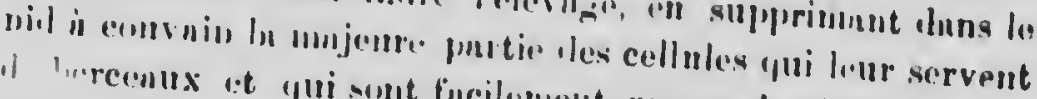

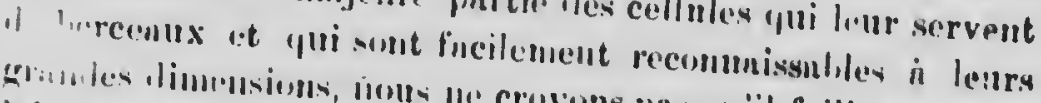

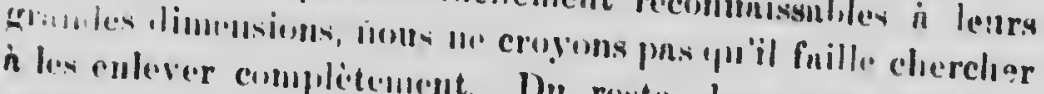

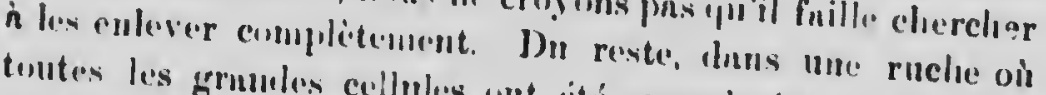

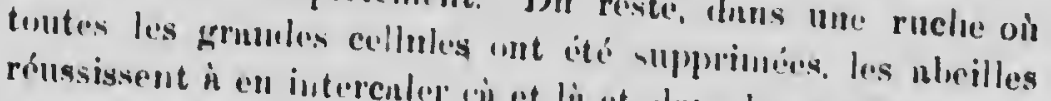

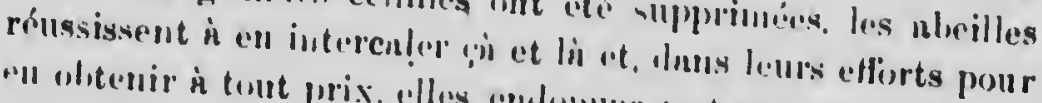

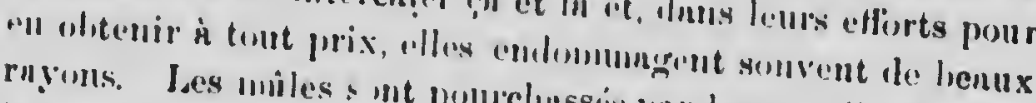

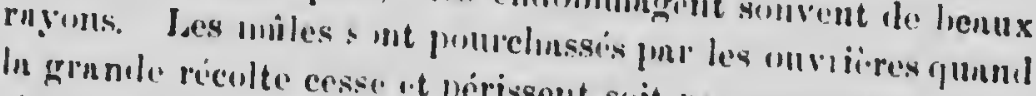

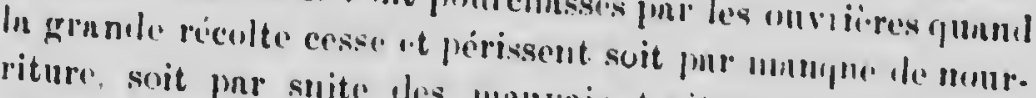
riture, soit par snito eles mnareis tmitoments quils ont

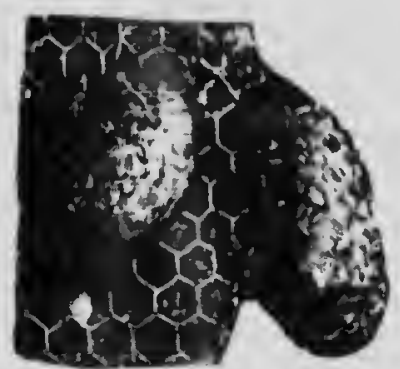

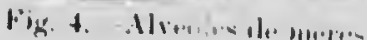

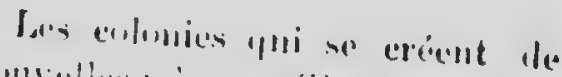

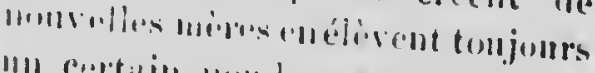
III certain momlare, sollrent 10 is

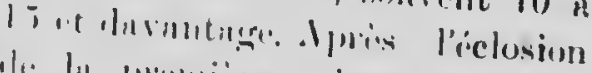

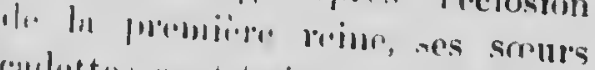

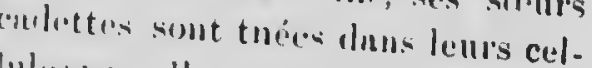

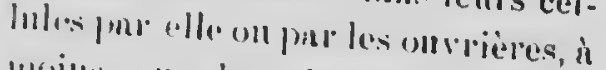
moilli fille ln colonire ue soit en proje. an liessiu d'essaimer; nlous

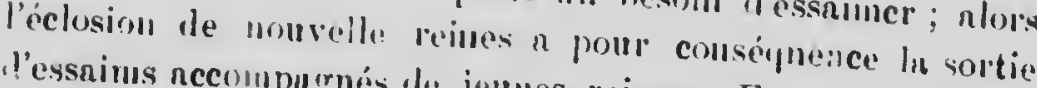

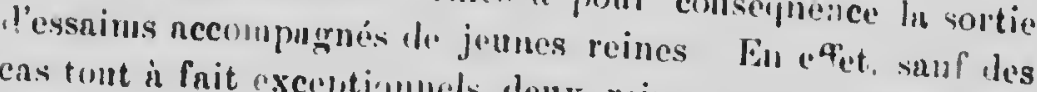
cas tout is fait axceptionucls, deux reines ne peurent exister siumltanément dius mue ruche, l'une des deux a-t ture par

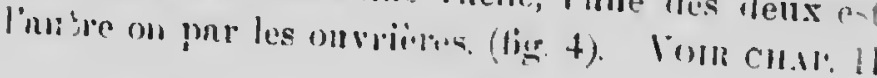

Jes ouvrieves, constituent In populution te lu colunie, et sont les plus petitos. Les mâles sont sensilulement 


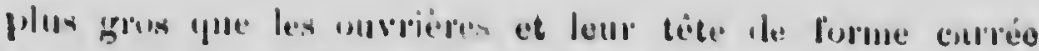

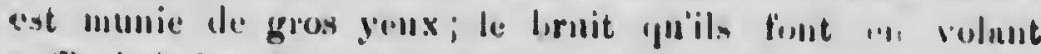

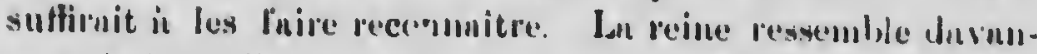

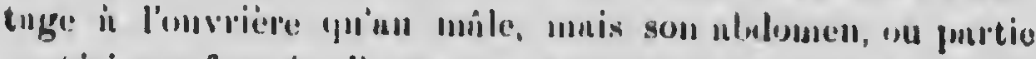

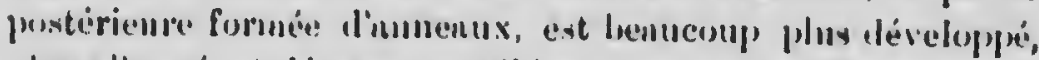
plus rllongri et dépussic sensiblement les niles. Sun corselet est nussi plus gros; ses puttes de lepriere ont une cunleur

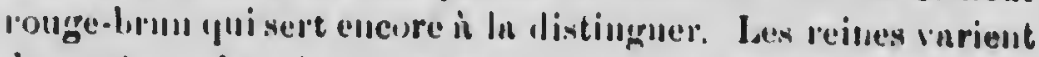

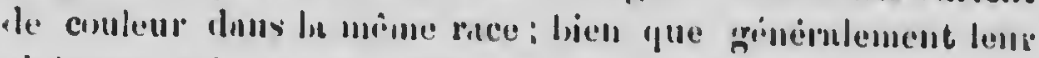

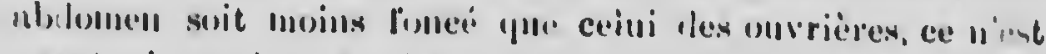

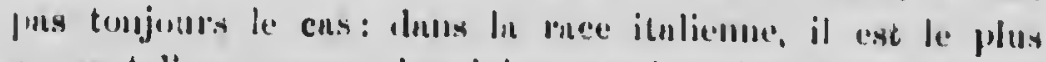

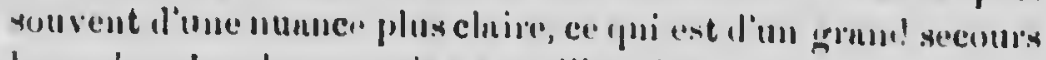

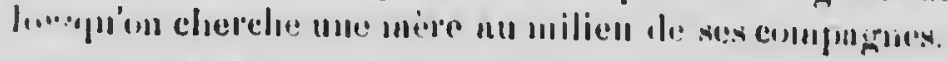

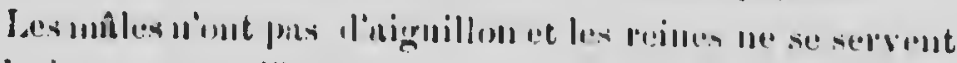
pass lu !eur contre linomine.

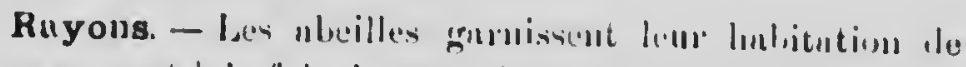

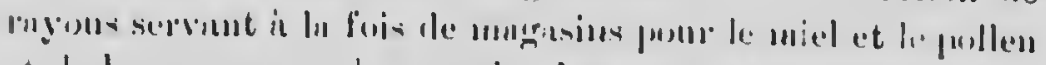

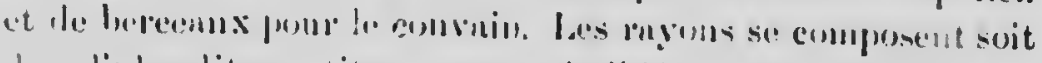

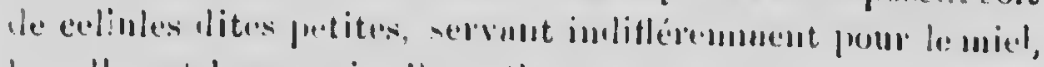

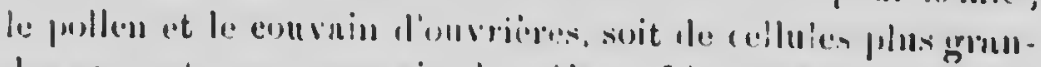

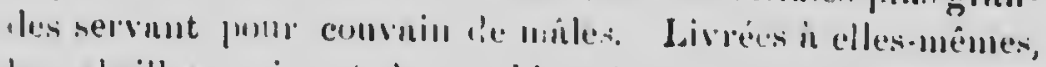

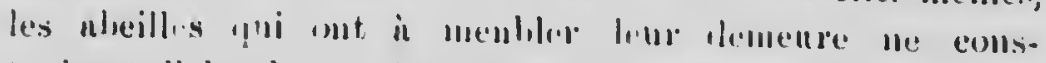

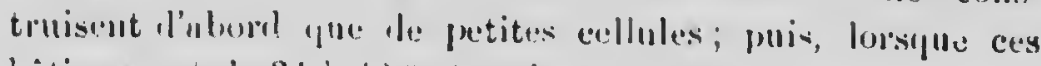

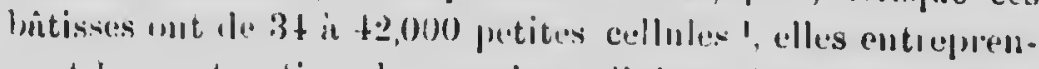

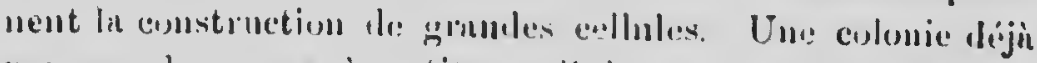

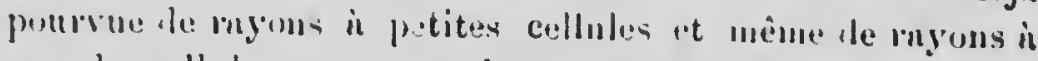

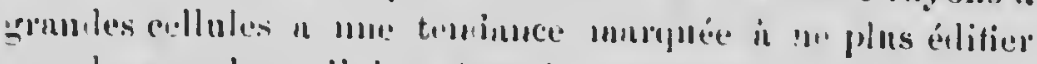
ylue de grameles celluhes, demet la construction sul plus vite.

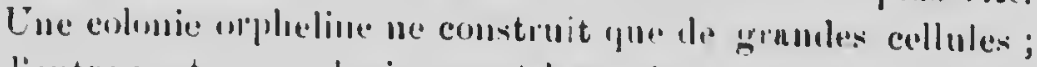
l'untre purt, une colonic "yount in su tête une: jeune reine de l'umee $n$ l'instinct de construire le preférence re petites

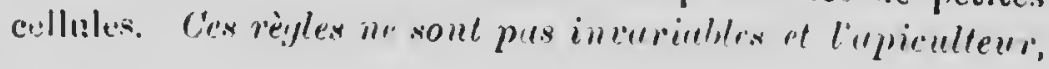

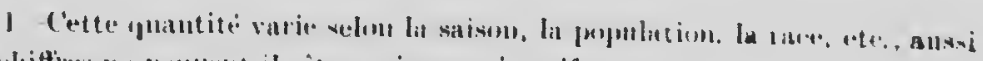

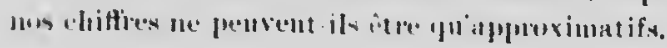




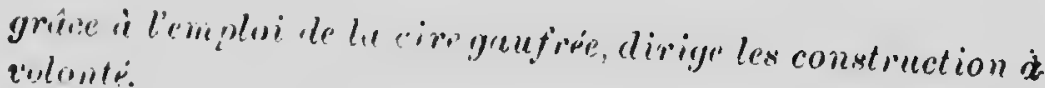

Los aluilles cur-truivent mee troisiènc espece decellules tempornires. lestinées in l'elerng des reincs et nffectant. comne nous liaroms lit plus hint, h forme d'un gland suspen lu an rapon lans nue pusition verticnle.

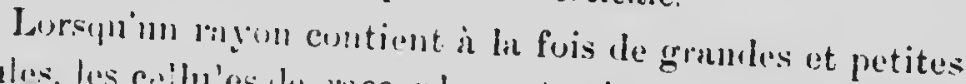
crllnhes, les collu'es de riaccorde ment. plus on moins irrégu-

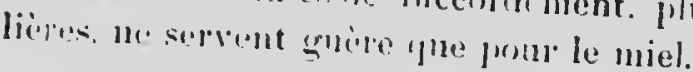

La cire ent me siereition din corps des aleilles. Elles

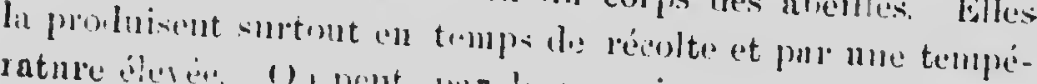

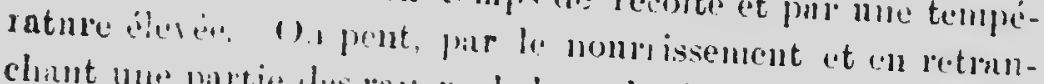

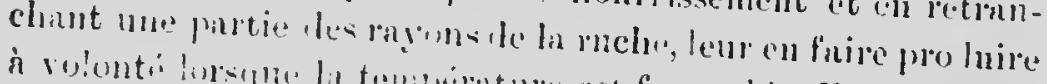

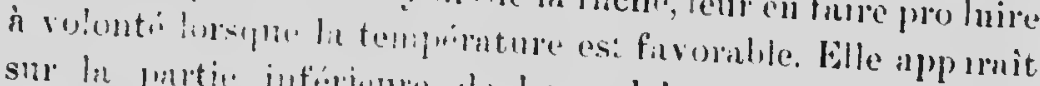

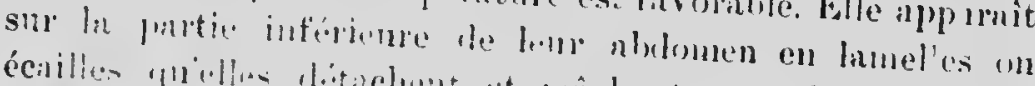

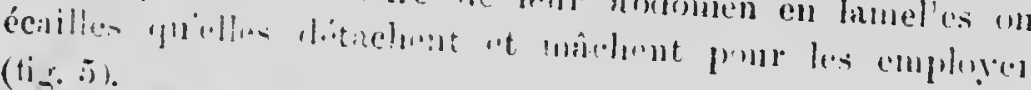
(iis.is).

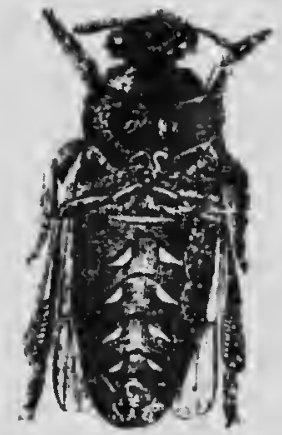

rig. it

Ia propolis tat me reime glu. he

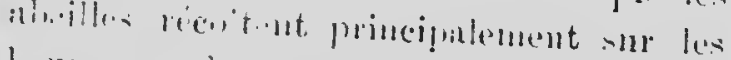

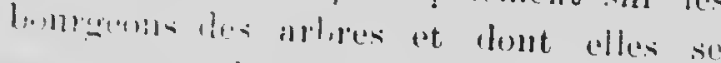

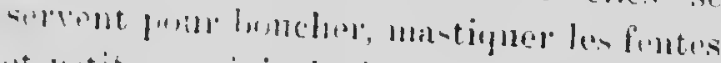
et petites asvitris le leur halitation, con-

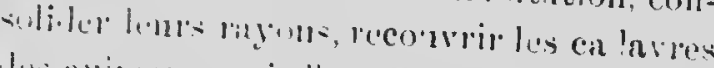

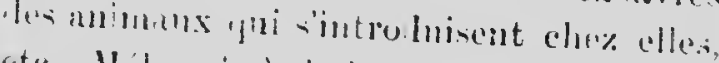

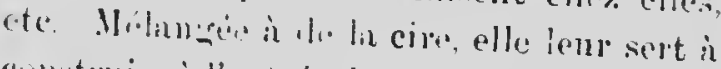
contmire a l'entrete de la ruche des traraux litem-if' contre lemrs cuncmis dn dehors. lille tran-portent cette résine, comme le Pollon, sur lenre pattes ab derriene.

La prophlis est fonc utilisée comme en luit, remis et

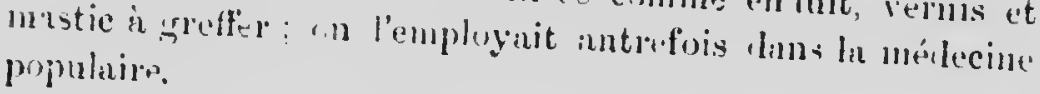

Le pollen, ou poussière ficmlante les Hills, sert prin-

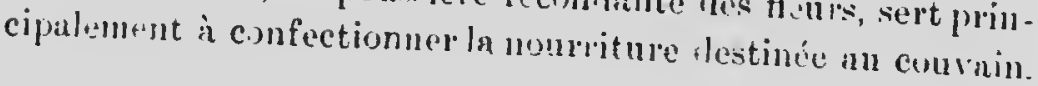


Les abeilles le trunsportent sur leurs futtes de lerriere et l'emmugasinent principalement lan: les tnvirm- lu couvain.

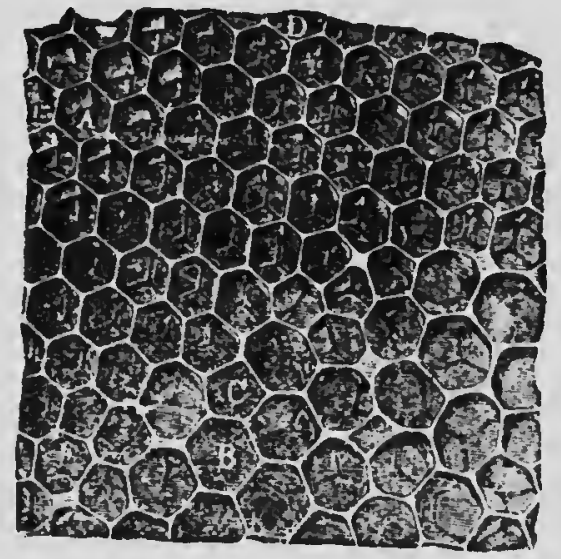

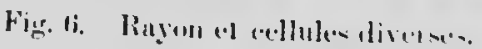

Rayous et cellulis diverses. - I,es det1x figuressuivantes repres. sentent ders pintions

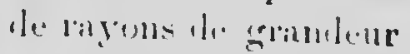

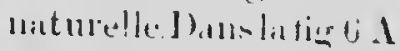
cot lalle fue tit. celluly à

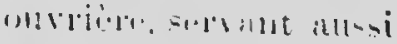

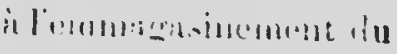
nifel it fullton Enli, ral loit ma cellule a mâle, scerout at $11 \mathrm{~s}, \mathrm{i}$

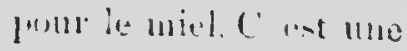

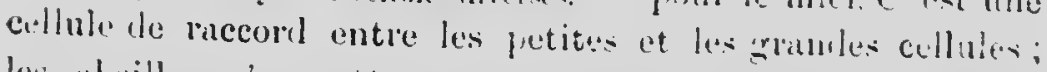

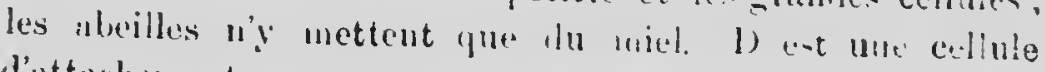
d'ittichoment.

Dills in tige $7 \mathbf{d}$ tist wue cellule royale riont la jeune reille est sortio: recellutent ; b ant uno cellule roginle ploercules contenant encarela jeune rein. ; le trait qui l'entume indigue nue manière de lécomper la cellult pour l'omploger ailleurs. ( et 1) sout des cellules royules co ur . incencées.

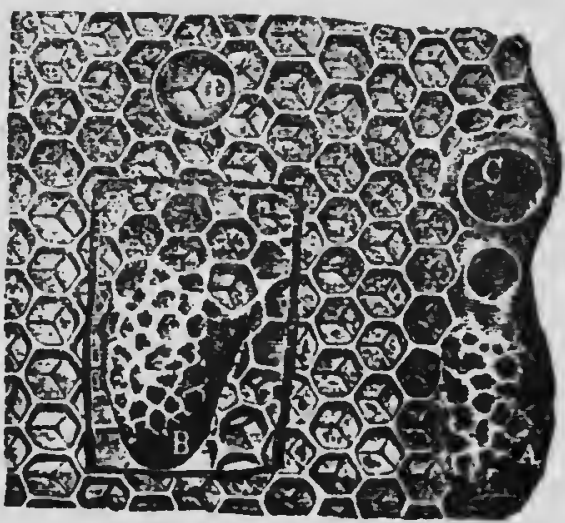

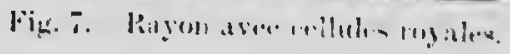




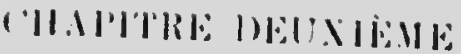

I.I lilisti.

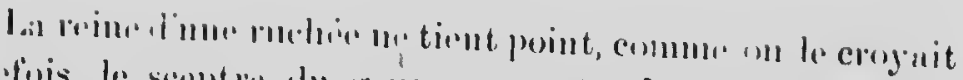

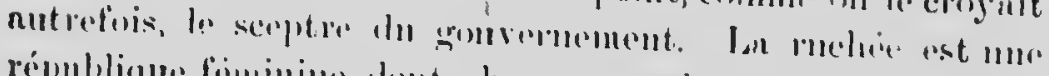

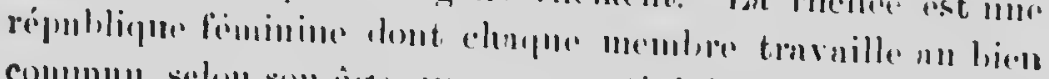

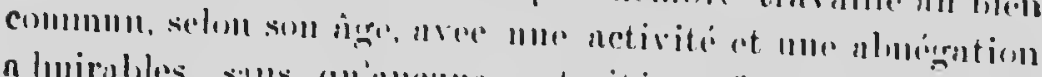

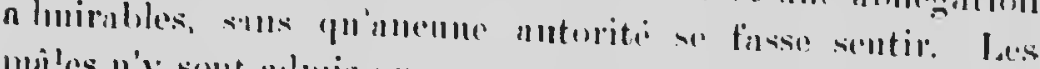

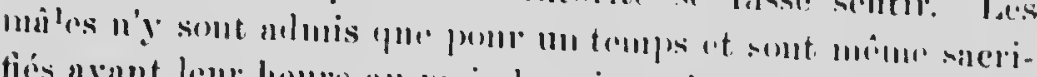

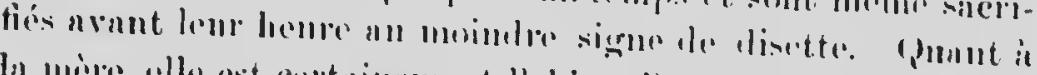

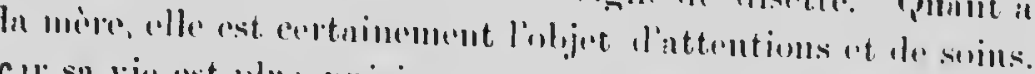

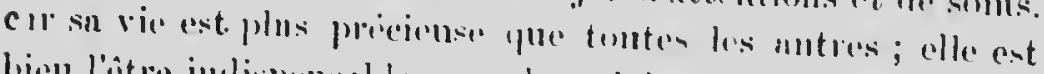

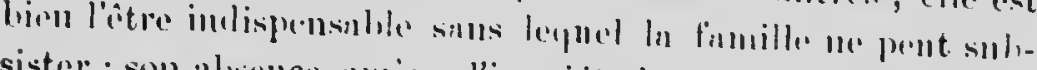

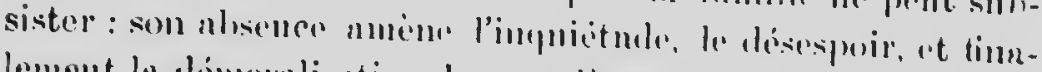

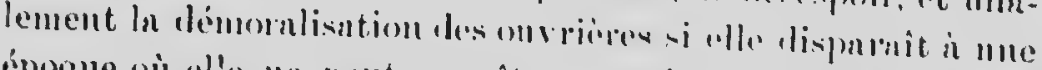

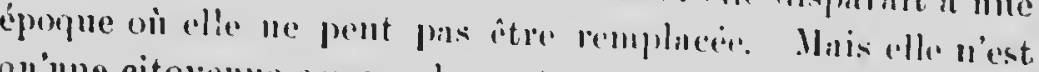

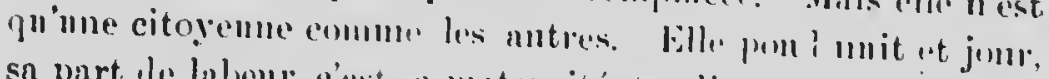

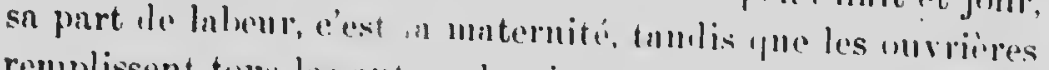

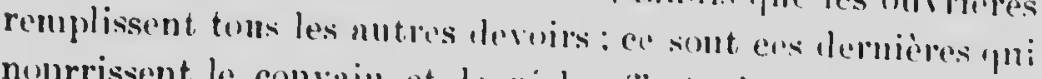
nomrrissent le couvain ot lo rechantent. de mesme priellos

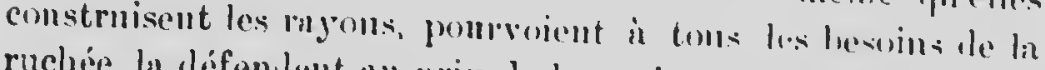

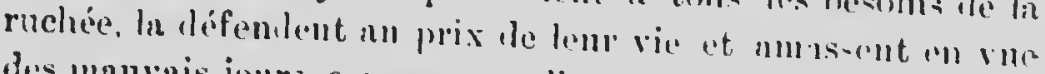

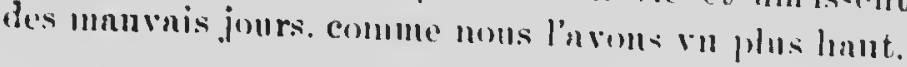

Si la reine ne sert qu'r unintenir la colenic daus mu etat de prospérite, il u'est pas moins vai qu'olle jout lall rile trios préponilérunt. Siuns elle, la colonie ne pent. manquep de disparaitre très rapilenent. Suns croyoni lonc levoir lui consacrer un chapitre spécial. 


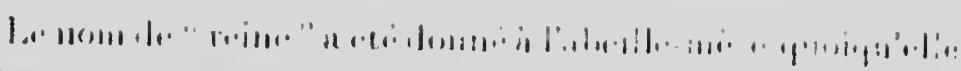

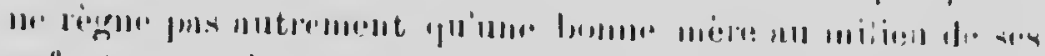

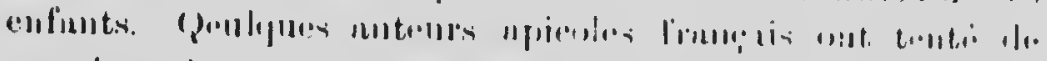

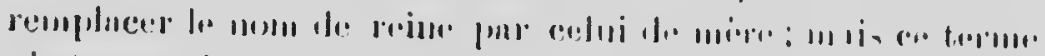

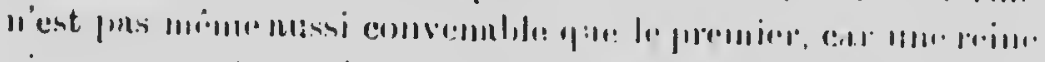

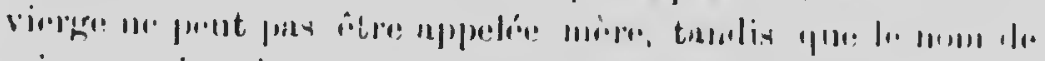

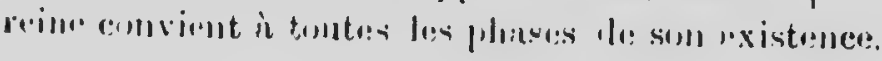

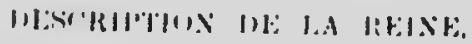

L.e linme de la reine differe sensiblemont de celle des autros aleillos; qusing blle ne soit pas, in louncoup pres, nussi voluminemse que le mîlo, som corps est plus long, beancoup

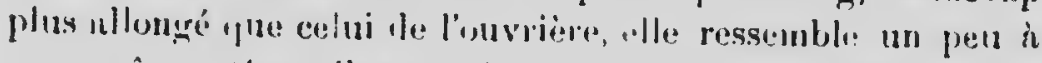

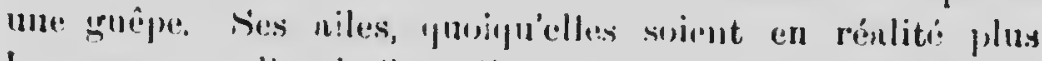

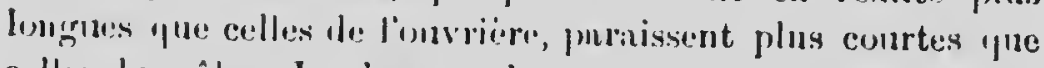

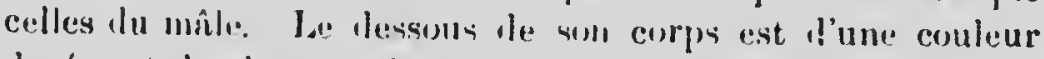
dorée, et le dossus, cho\% ln reine de rice commonno, est

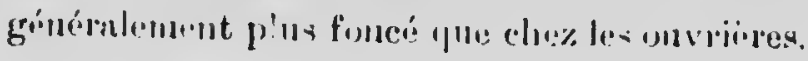

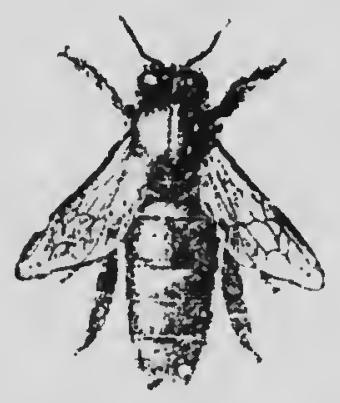

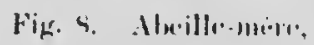

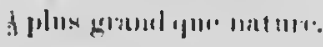

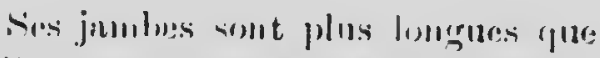

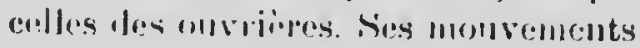
sont lunts, semblubles it cenx d'une matrome qunul rien us ln lérungre;

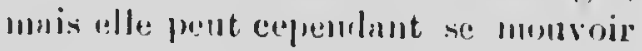

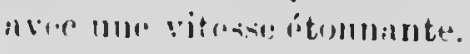

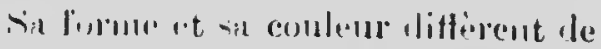

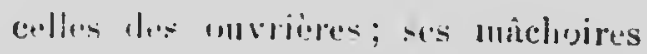
soll, flus comtoss, sal trite est plus

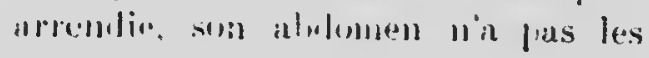

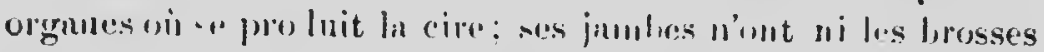

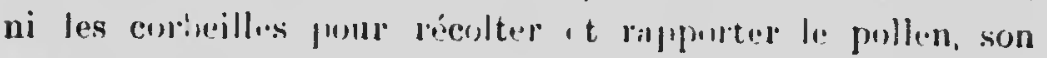

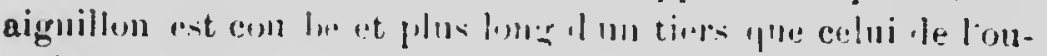
vrière (liger 8 ). 


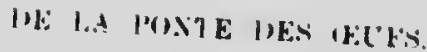

La reing est lu senle femelle purfaite exi-tant dans la ruche. Pondre est sn sende fonction et elle s'nequitte si bien de cette churge qu'il n'est pas mre de troured des reines

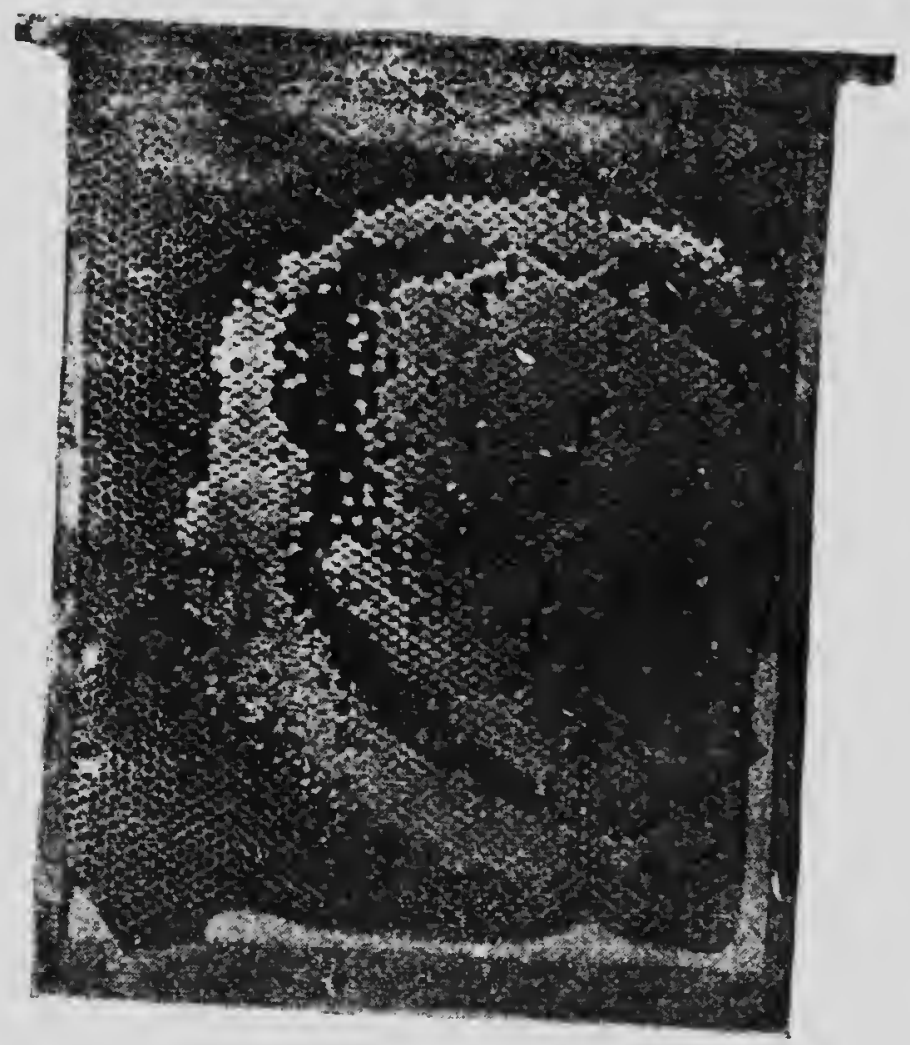

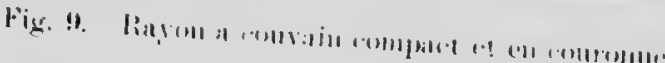

Letrou"

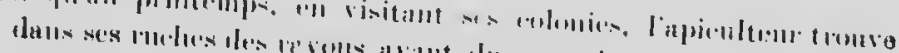

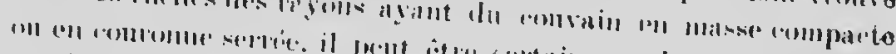
1หun lenise.

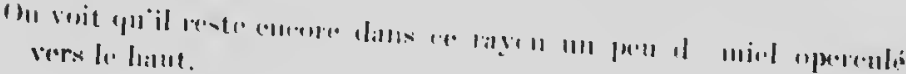


poudaut 3,500 celfis par jomr, pendant des semaines consécutives, lurant la bonne suison." Dans les ruches choloservation, l'on a vn les reines pontre six cenfs it la minute. (rig a)

La reine pond en raivon de la nourriture quelle regoit des ourrieress et dans les eellules que celles-ci mettent in sa disposition; ce sont llonc las onvieres phi reglent la ponte

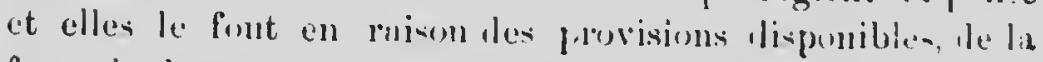
force de la population at a les circonstances extérienes si

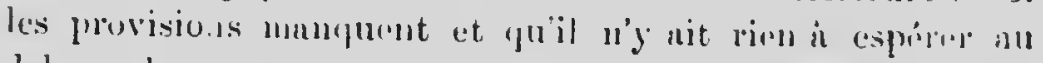
dehors, la ponte se restreint on s'arrete; et allum, lan- les cas de grande lisette suldite (les abeilles pus plus que liup-

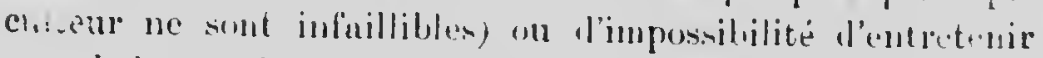

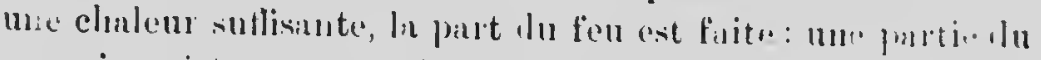
collvain existnut ent sncritice et jetie lours le la raches nuses que les sucs utilisulles on ont été extrnits. vii, an contriniru, les vires se manquent pas et que les npports nomvanx soient alomlants, lis alueilles stimulent la poute de lit reine en la nourrisant davantigne.

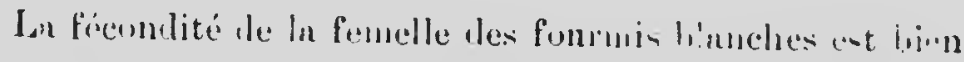

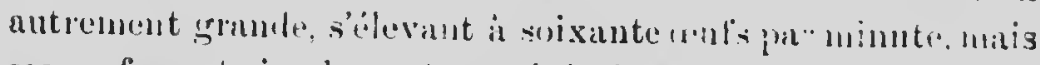
ses renfs sont simp'enent expulsés de son copps les onvrieres se chargennt de les placer lans les nourriceries combunaldes; tandis que la reine dépune les siens, 1 in an, daur les cellules

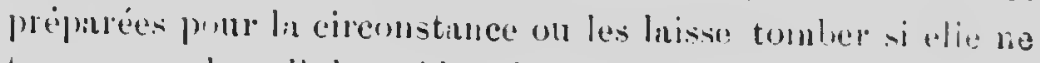

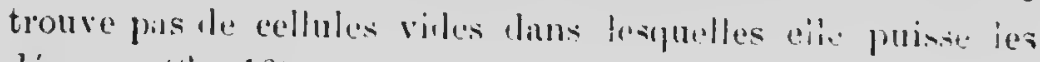
lriposer. (tig. 10).

L'e nombre de :3,500 is 5.000 antes, quine hume reine peut pondre en 2, heures, foum sembler exugere anx culteurs qui n'unt que le petites ruclues Ils demanderont

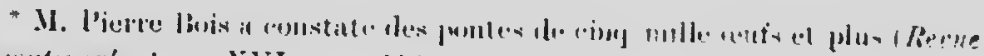

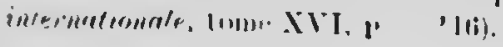




\section{- $21 i-$}

peut-itre communt an a plu so resuire compte fluse telle

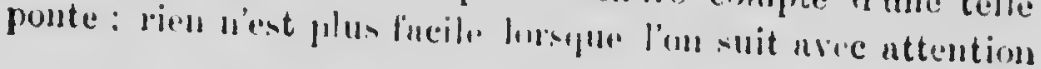

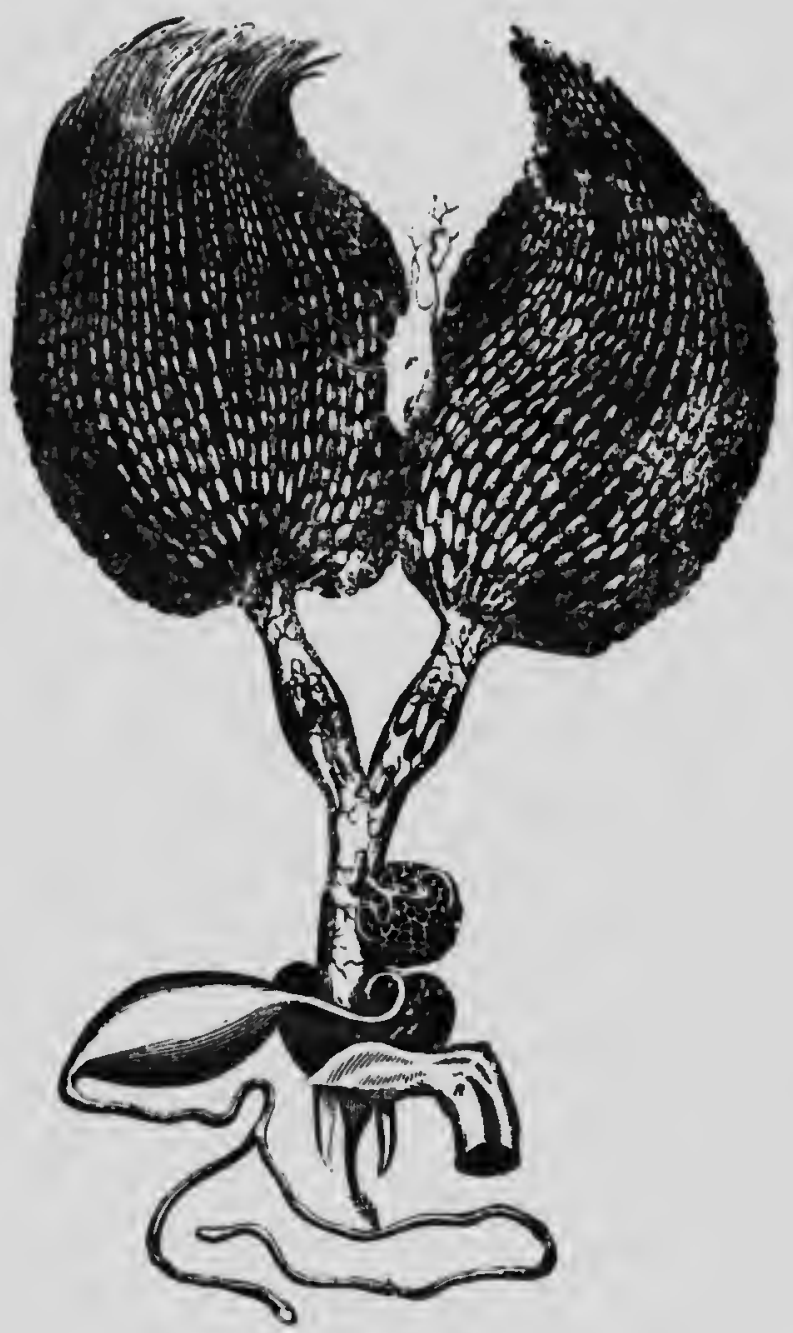

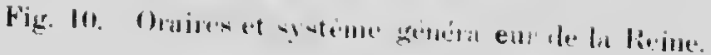


le renouvellenent de l'essaim et pre l'un snit la conte lurée de In vie de lionviriere.

Des pontes nussi aboulantes peuvent être viritiés chaque annéc dans la plupurt des bomncs colonies, pemlant le

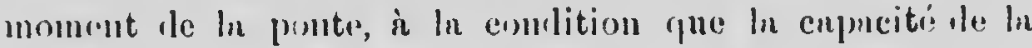
ruche permettre is la reine le léployer tonte sa frementite.

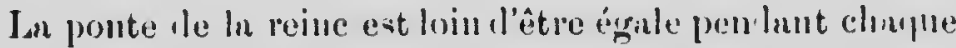
mois de l'année. C'est is la tin dn printemps qu'olle pund le plus et pemlant les mois de l'été, nvant le monent de in gramle récolte et tant qu'elle lure, sn ponte diminue pour cesser in ln fin le l'éti; plus tôt on plus turl survint lin lntitule.

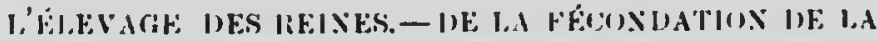

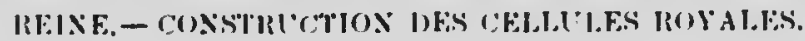

Nons allons maintenant léerire l'élevion les reines. Quand, dans b belle saison, une col mie levient tries populeuse, si ses ulieilles se préparent à essuimer, elles construisent in certain nombro le cellules de reines,qui sont généralement

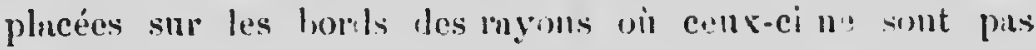
attachís anx cités de ln ruche. Ces c llules ressemblent un

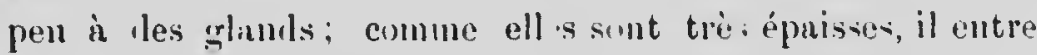
relntivement beauconp de cire lins leur constraction. (Jn les rencontre raremment entic̀res, les alreilles les liminnant à la dimenion de la cupule d'un gr!nul nussitît que la jeune reine en est sortie. Ces collules, penlant le développement de leurs laves, sont l'oljet de heauconp de soins de ln part des ubeilles. Il ne sc pisse presigue pas t1 re seconle sans "̨u'unn ouvrière n'y introluise ln tête, et ì peine cll est-ellc sortie qu'une autre y regnrile $\dot{x}$ son tour, soit pour roir si la larre grandit, soit pour ougrmenter sn provision de nourriture. On comprenlira nisément les quelle importance ces cellules 


\section{$-28-$}

sont pour In communnuté, eu ley voynut le centre d'une si grnnile att "nction. (fig. 11.)

Comme il vaut miens que les jeunes reines ne soicut pas toutes du wême îne, les cellules pọnles ne sont pus

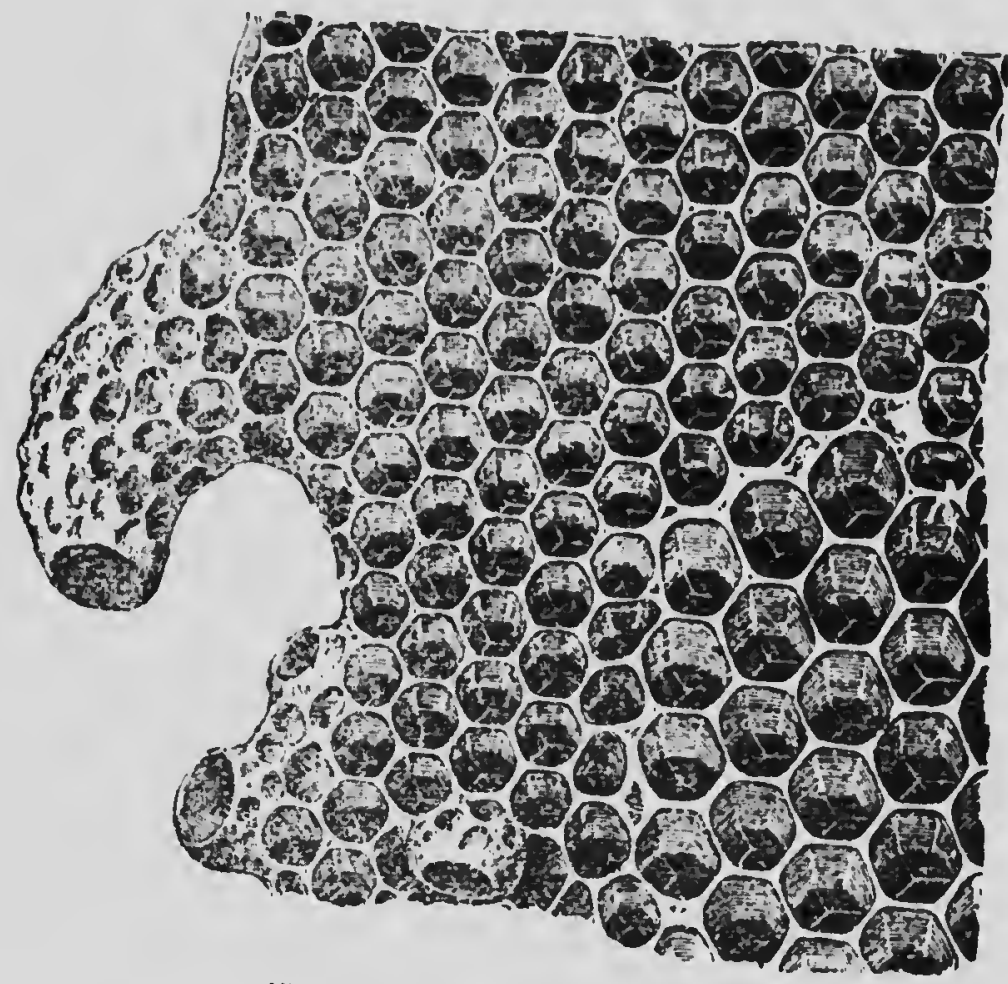

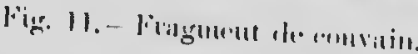

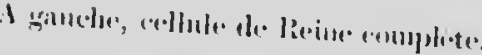

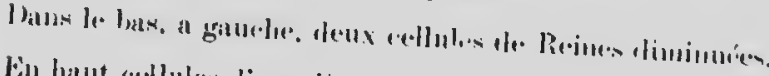

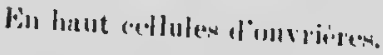

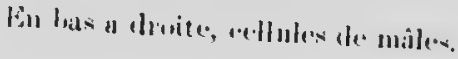


prépnrées ell même temps. ()n ne snit phs bien exnctement comment les (eulfs sont lépo és lhus ces cellules. Nous arons

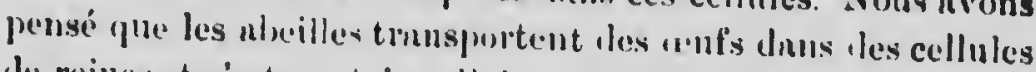

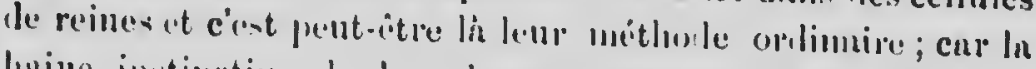

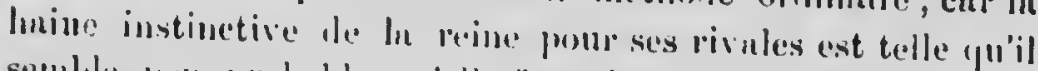

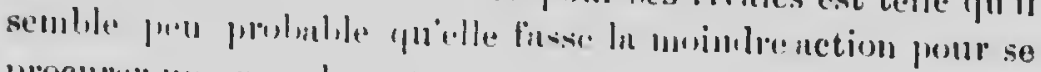

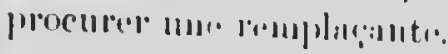

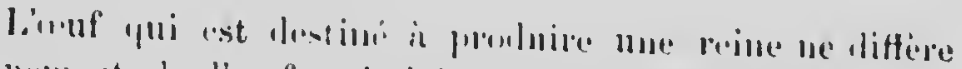

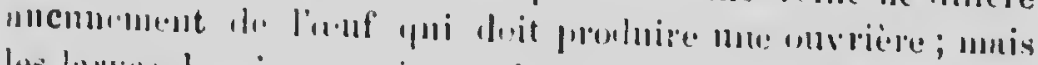

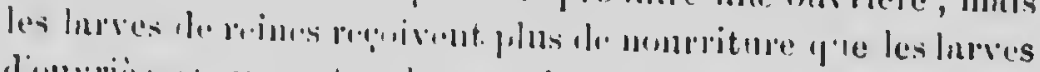

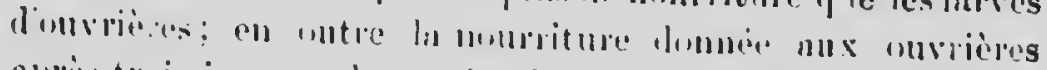

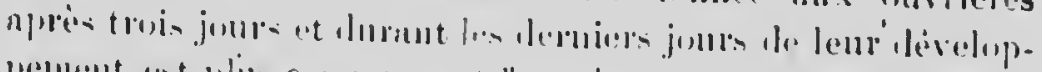

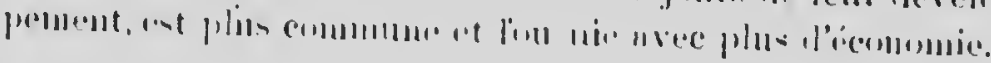

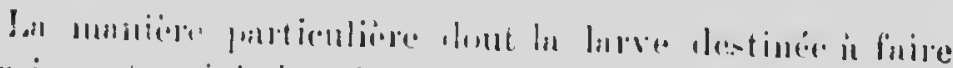

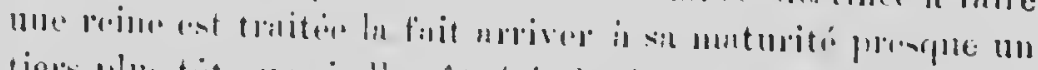

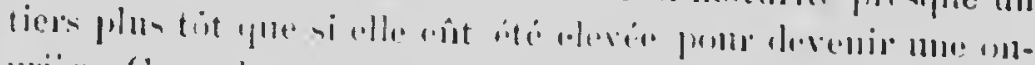

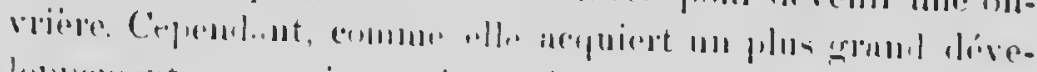

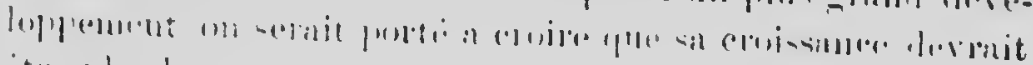

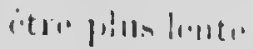

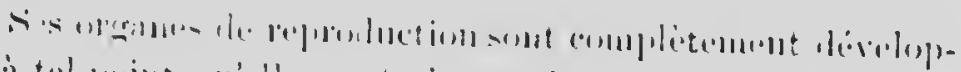

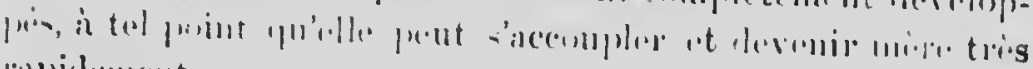
pappistencent.

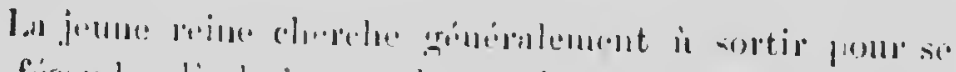

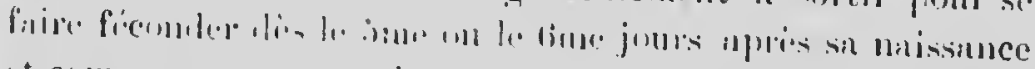

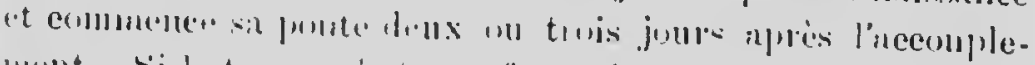

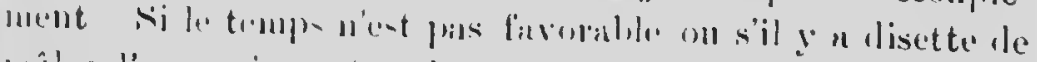

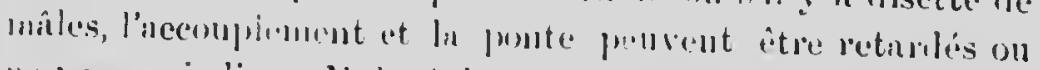

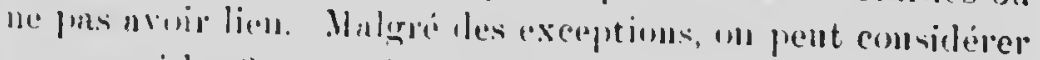

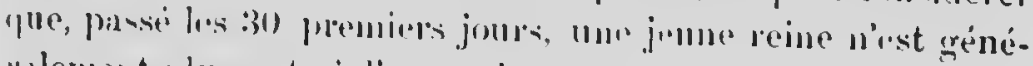
blement flus ilgte it linecouplement.

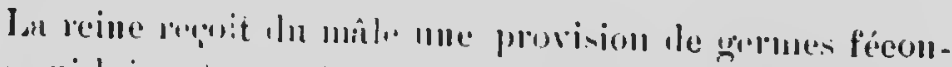
lants qui lui sert pron thut. son existence : ces germer sont recous dons un petit sac, lent l'oritice est sur le pussage des "ufs a leur descente der wrires, et selon que la reine a a 


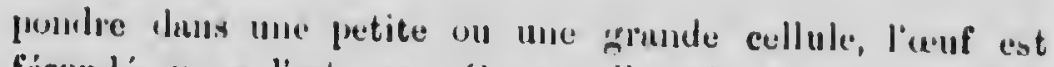
féconile on ne l'est pas. On nppelle reines bourdonneuses celles qui ne pondent que des anfis mîles; celn provient sojt de ce qu'elles nount juns été féconlées, soit de ce que leur provision de ğemos fécomlants est épuisée. D'untres léfectuosités dans les orgunes de ln mere ont pour eftet de lui faire pondre une proportion démesurée le mâles. (tig 10).

\section{W'UF:E: IH: I.A VIK IIE LA MEINE.}

Tumdis que ln vie des ouvrieres est limitée in quelques mois dhus la salison morte et in six on sept semulnes en moyenne duns ln snison dinctivité, par suite de leurs rudes labeurs et des nombremx dangers nuxpuels elles sont expo-

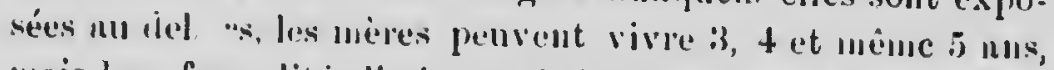
mais leur fecondite diminue armémlement des in troisiome anme et, les ubcilles ne les remplaynt que lorsqu'elles deviennent reeflenent importentes, l'mpicultenr de profession

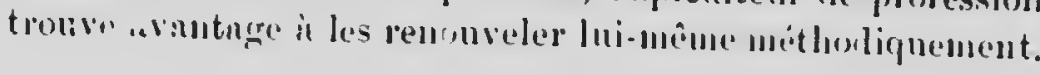

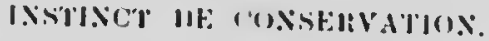

Lee proédé les abeilles pour élever des reines lors d'une néces-ité spéciale est encore phas merveilleux que ce que nous. venons de lécrire. Si les nbeilles ont des aufs l'onrieres ou des larves niunnt pus plus de trois jours, elles construisent une large cellule tenunt la place de trois, en rongennt les trois cloisons qui leur sont communes. Apres aroir détruit les ruf's on les lares de leux cellules, elles doment i ln lave eonscrvé la nouriture labitnelle des larves de reincs et par cet élnogissement de l'espace elles lui procurent ln place néce-saire ì un plus grand déveloipement. Pour assurer le succès, elles préparent d'ordinnire un bon nombre de cellules, mais souvent elles ne continuent le travail que sur quelques-unes d'entre clles.

Les arufs éclosent trois jours après qu'ils ont été pondus. 


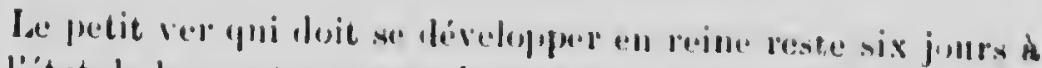

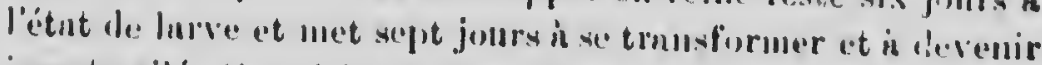

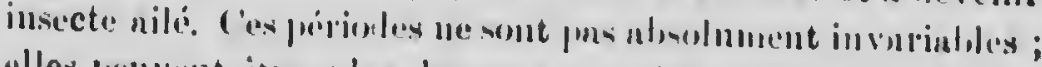

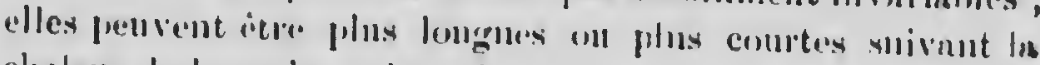

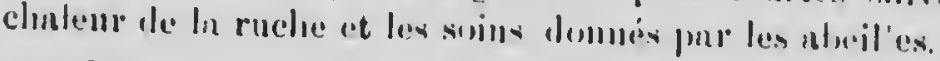

Il funt anuc de dix is sei\% jums. dix junrs si la larvo

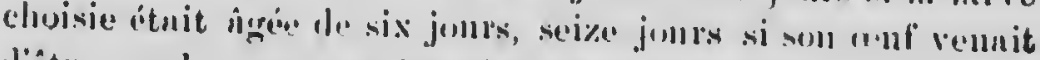

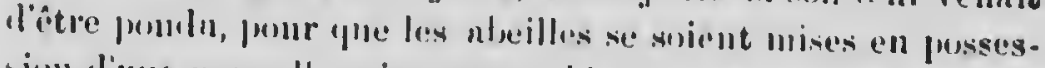

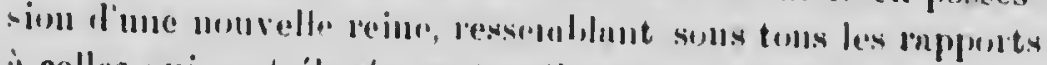

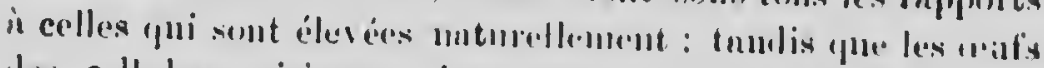

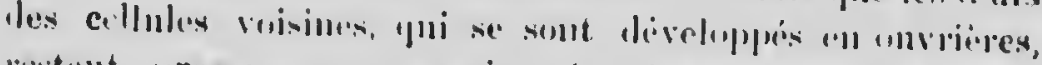

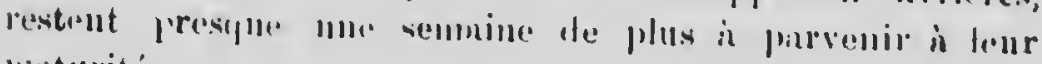
unturifé.

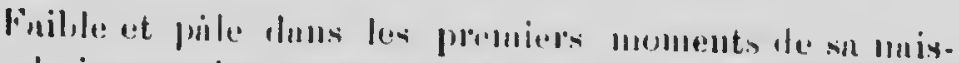

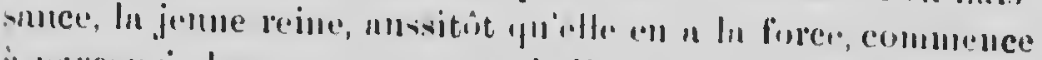

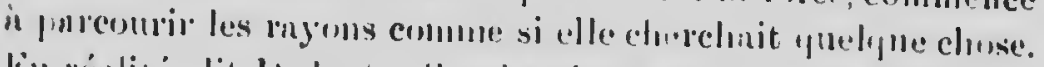

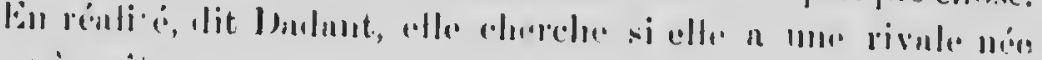
(1) it unitr.

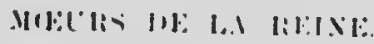

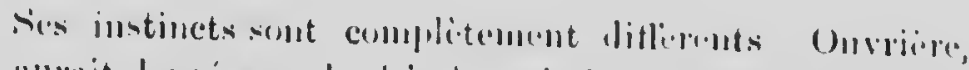

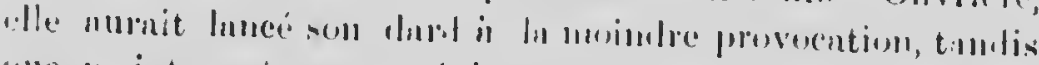

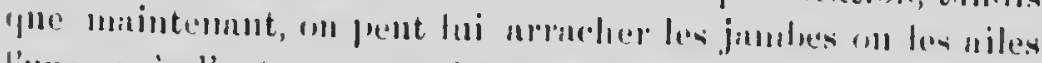

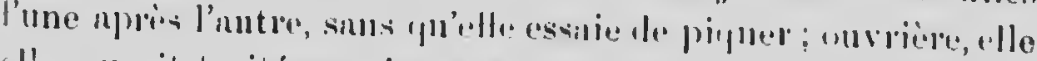

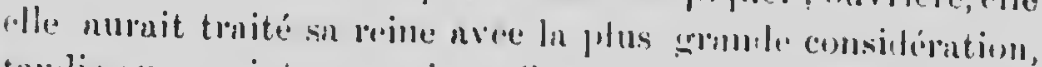

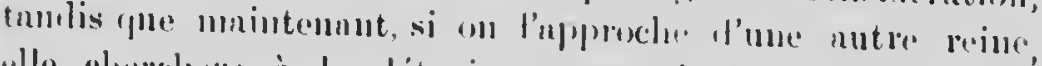

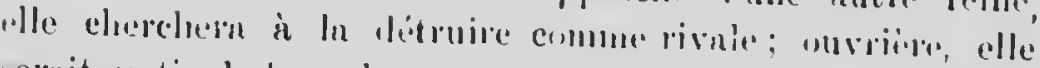

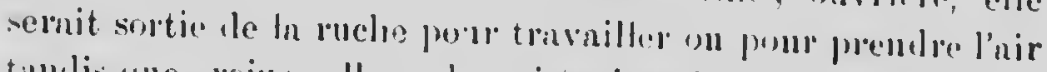

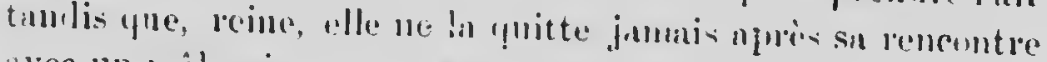

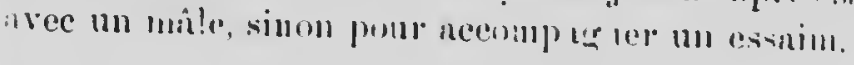

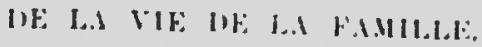

Ln rejue est troitce arre nutunt de respect yne l'utfection

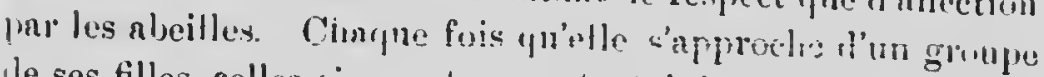
le ses filles, cellos.s: se rot..'ruent at lui montrent de liffie. 


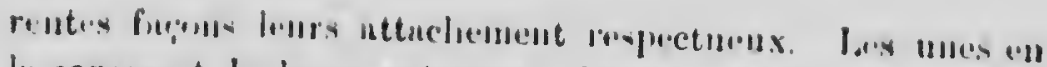

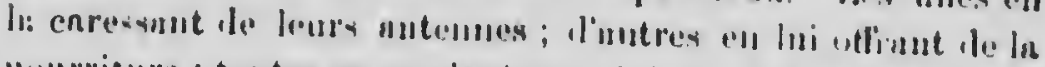

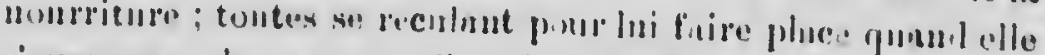

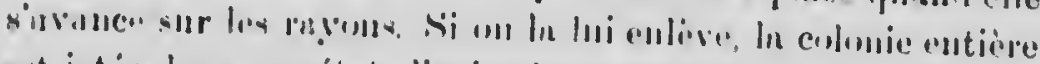

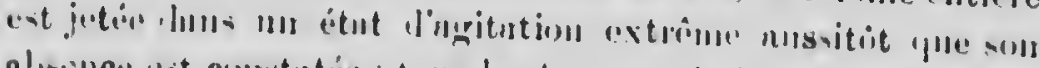

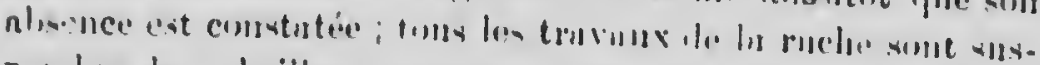

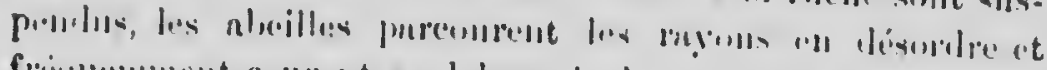

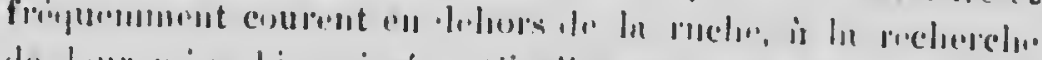

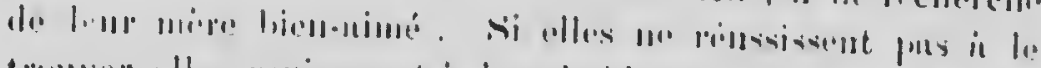

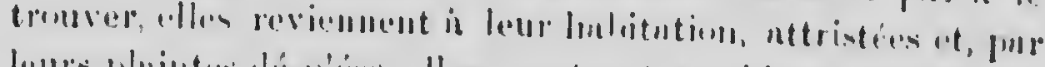

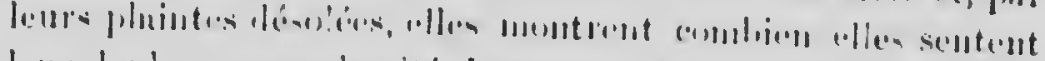

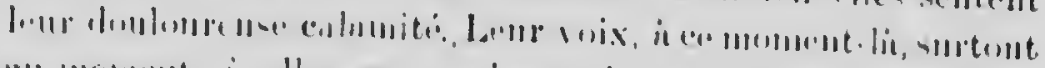

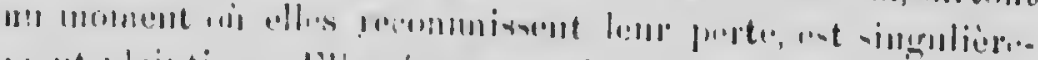

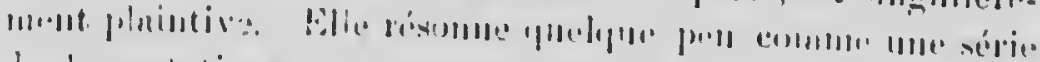

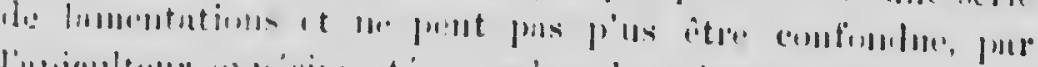

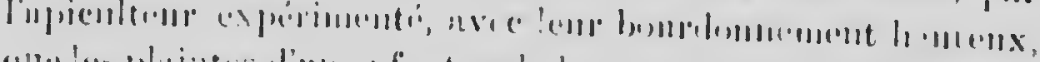

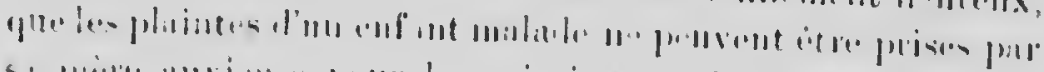

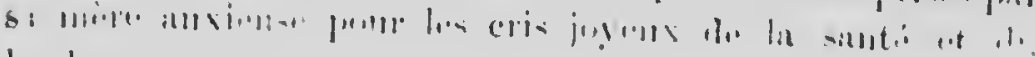
Insthellt. esporitidise:

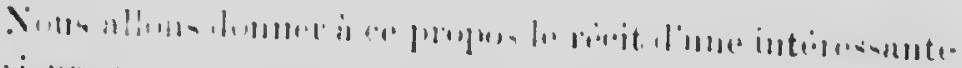

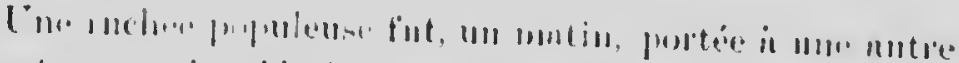

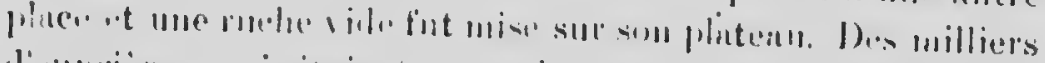

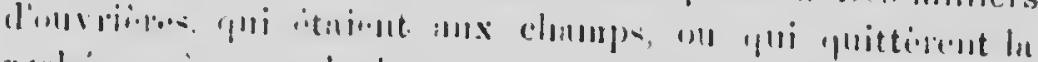

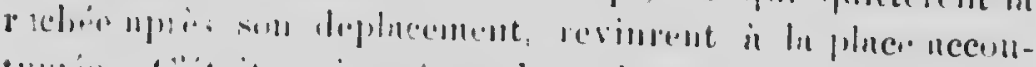

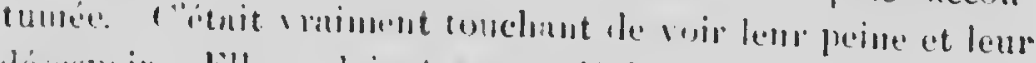

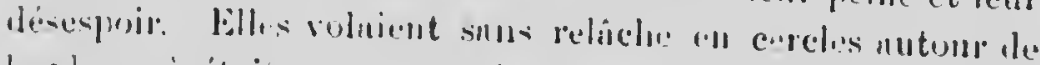

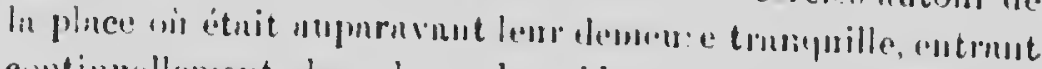

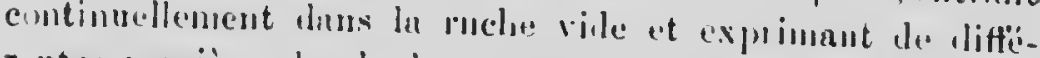
r.ntes manieres la louleur qu. lan comsuit une si cruelle dépussession. Vers le soir, cessunt de voler, elles elltritent

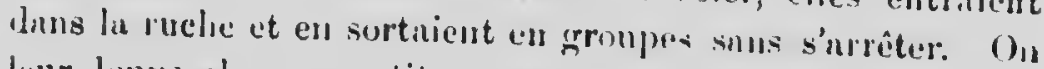
leur lonna alors un petit morcenu le comvin contenant des

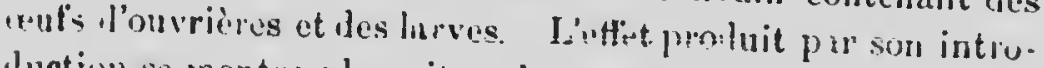
luction se montia plus vite ruo'on ue pourrat le lire. Celles 


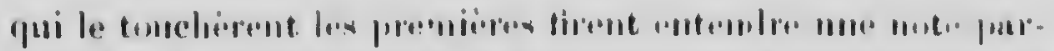

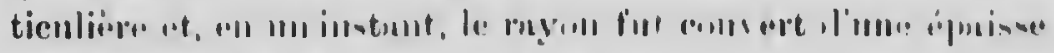

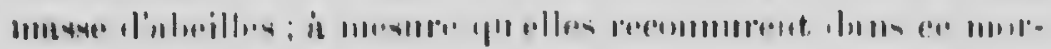

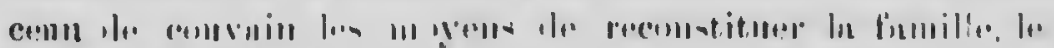

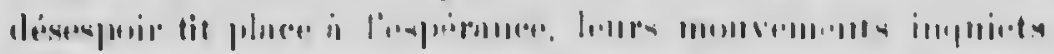

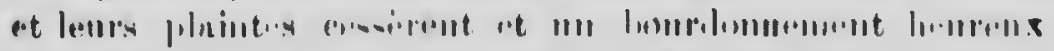

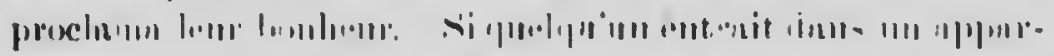

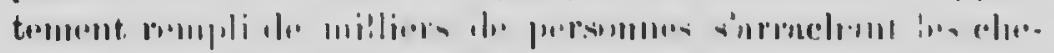

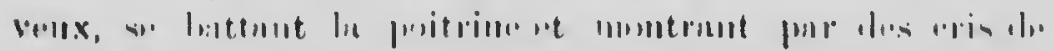

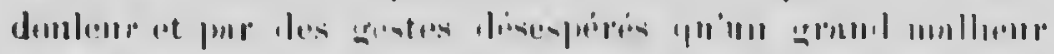

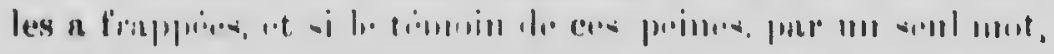

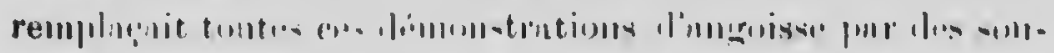

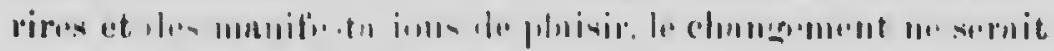

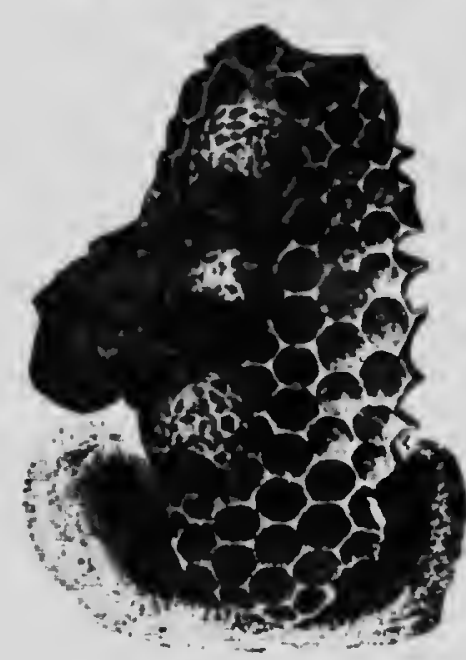

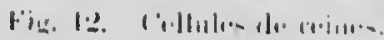

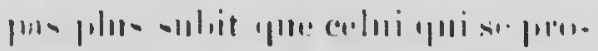

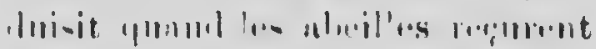
(… ry

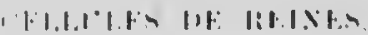

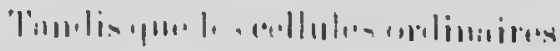

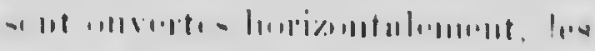

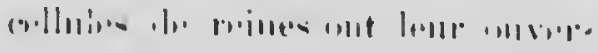

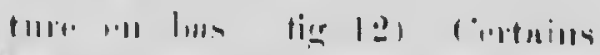

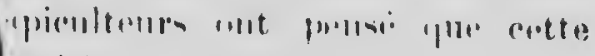

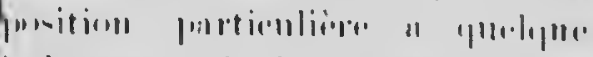

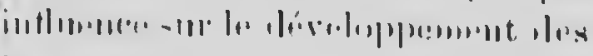

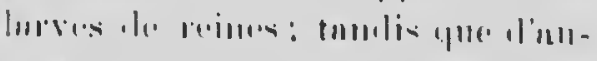

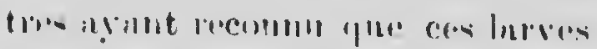

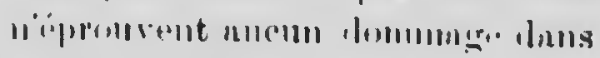

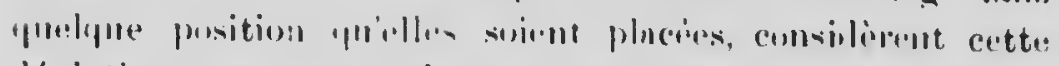

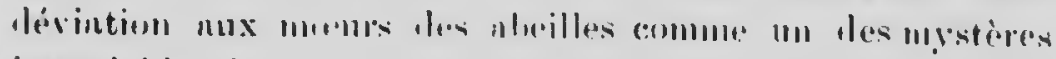

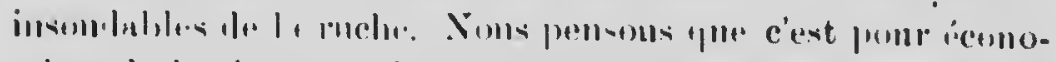

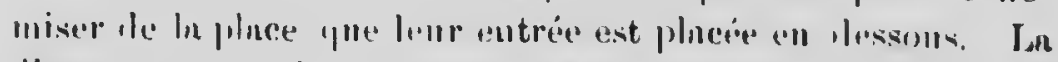

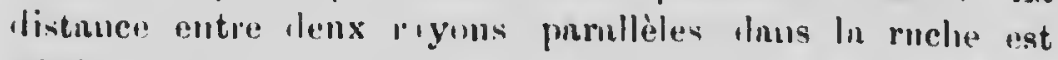
géuémlemnt trop putite pour que les cellules ullomgrang iles reines puisiont sumvir horizontalement sums se trourer en 


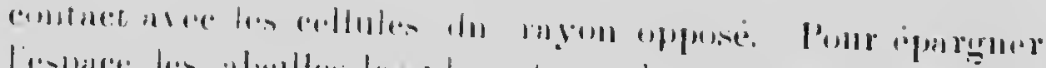

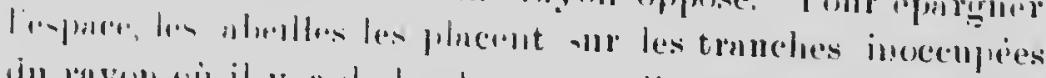

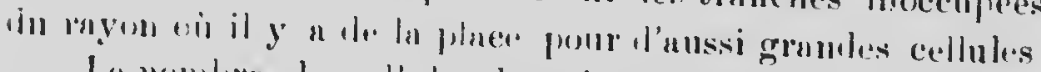

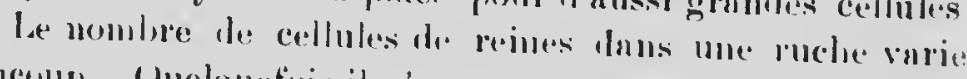

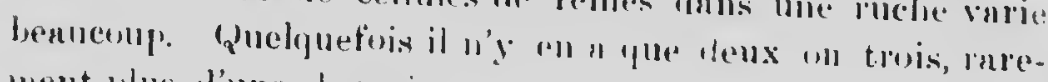
ment jus l'une demraine.

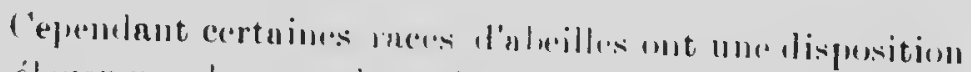

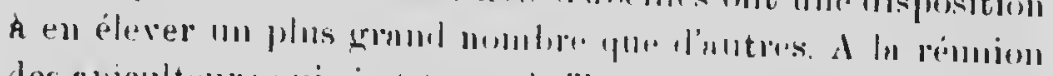

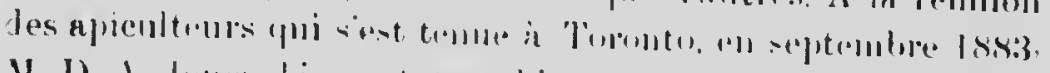

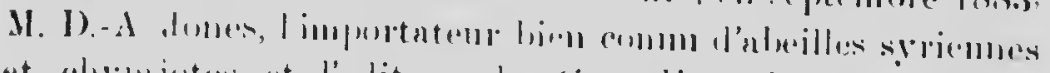

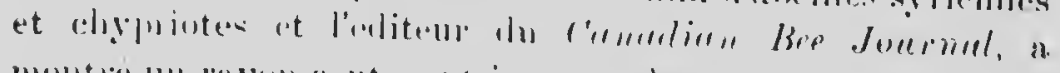
mont

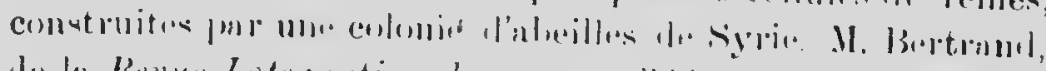

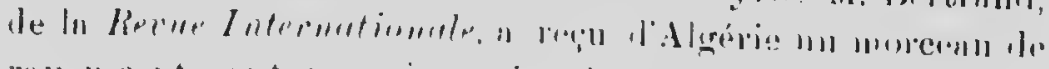

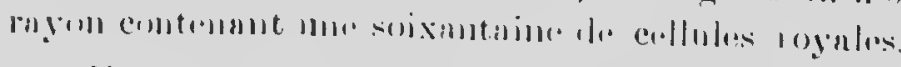

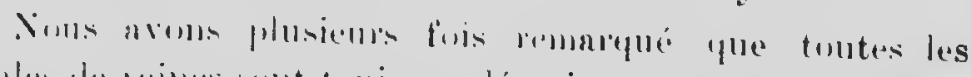

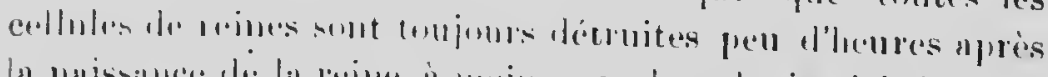

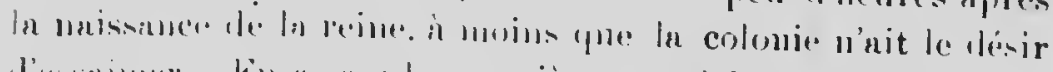

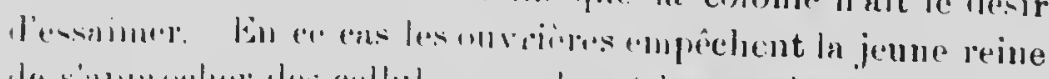

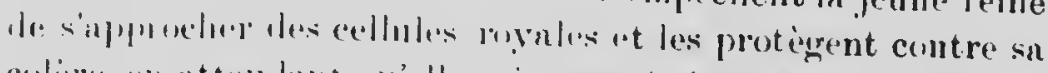

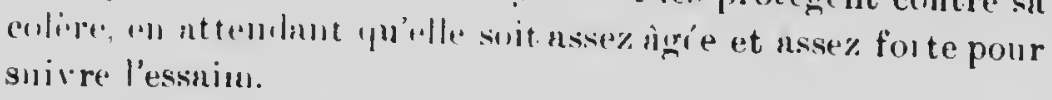

Quelquefois doux reines quittent leurs cellules au même instant. loici une narration de Huber, qui a été témoin d'unc pureille circonstunce :

"Le 15 mai dit-il, deux jeunes reines sortirent de leurs cellules presque nu mêne moment, dans une de nos ruches les plus minces. Dès qu'elles furent à portée de se voir, elles s'éluncirent l'mue contre l'nutre uvec l'apparence l'une grande colem at s. mirent lans une situntion telle que chacune avait

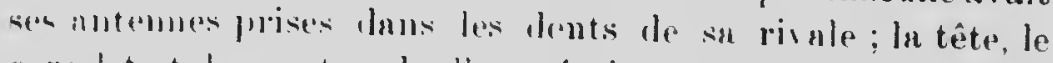

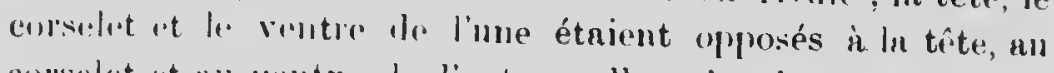

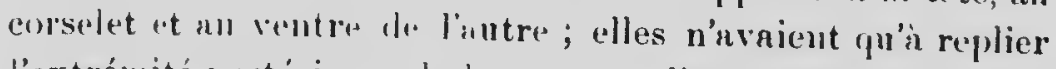
l'oxtrémité fustélicure de lenr curpes, elles se serajent percées réciproguement de leur niguillon et seraient mortes tontes 
les deux duns le combat, muis il semble que la nature nin pus

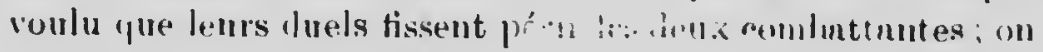

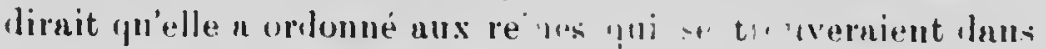

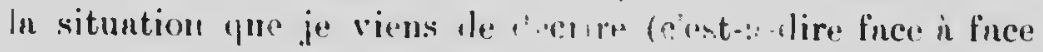

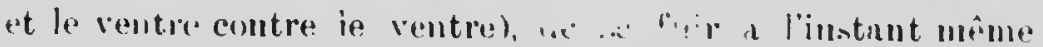

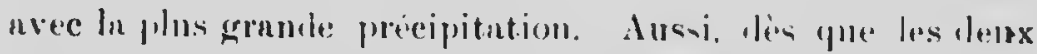

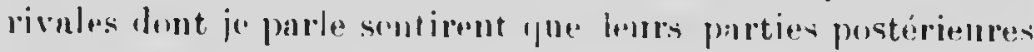

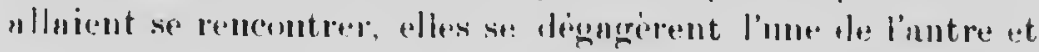

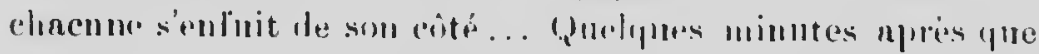

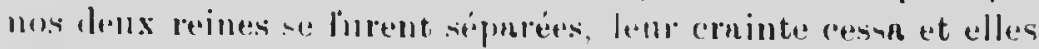

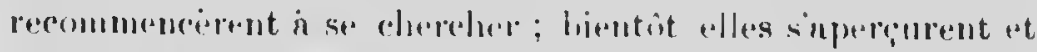

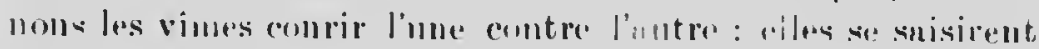

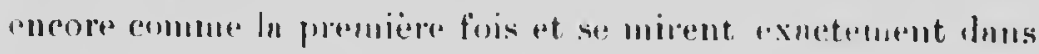

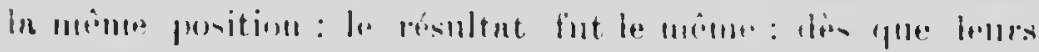

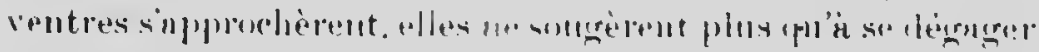

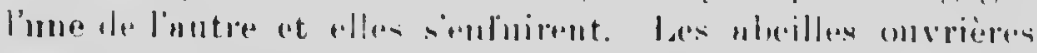

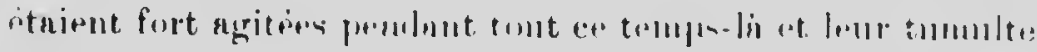

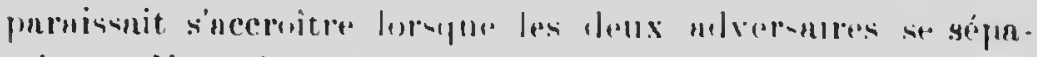

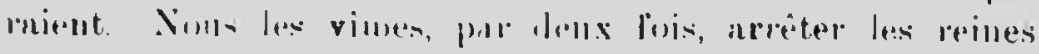

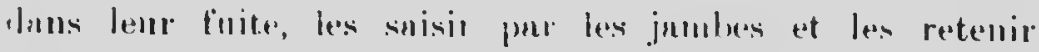

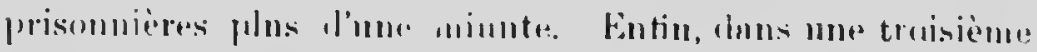

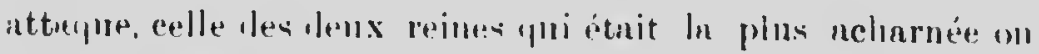

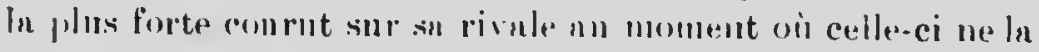

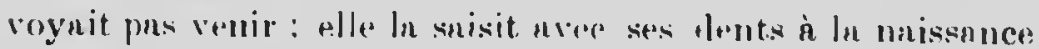

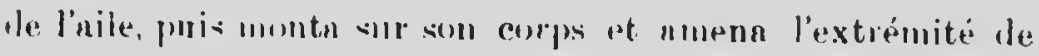
son rentre sur les depriers nuneanx le son enuemie, quelle purvint facilament ì percer de son nignillun : elle lincha nlors l'nile qu'ell. tesnit entr\% ses rents et retirn son larl: In reine vaincue tombn, se trninu lnnernissmmment, perdit ses forces tries vite "xpirn lientit npris"

Quoique denx reines ne puisent halitur ensemble la minm ruche, cepremlant. il arvive jmofois yue ln mère et in fille soipnt trouvée virant en paix lnus ln même colonie at

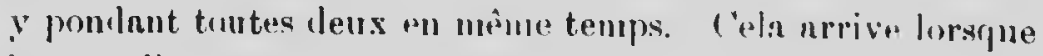

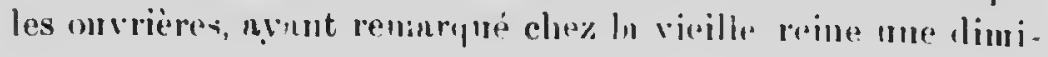


nution de fécondité, en ont élevé me jeune pour la remplacer. Mais cet état anomal ne dore que quel. pues semanes, lanement plus.

Une autre fois nous cherchioms, flit Dadant, pour la rensplace'r moe reine dont ln fécondité wrait lininué; ǹ notre - grmul étomesment, la rache était pleine de combin. Nons tronvinmes la vieille mire ; évilemment une reine si petite, si mnigre et si vieille ne pourait itre la mère l'une si nom. brense prougéniture. Nous continnumes nos recherches et hientôt une autre reinn. fille le la première, grosse et grasse, parnt levant nos yeux. Sii après noir enlevé la veille reine que nous nvions trouvere la premiere, pensant aroir rendu la ruche: orpheline, nous avions lenue a la colonie une nutre reine, les abeilles l'nurnient létruitr immélintement.

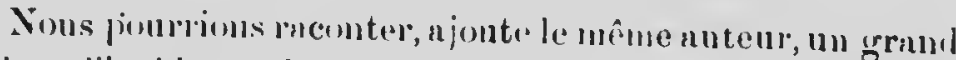

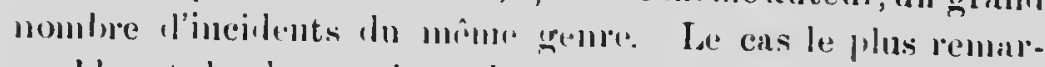

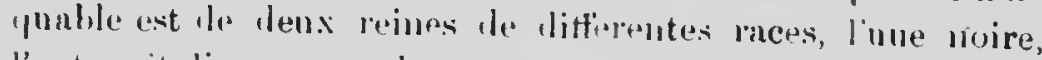

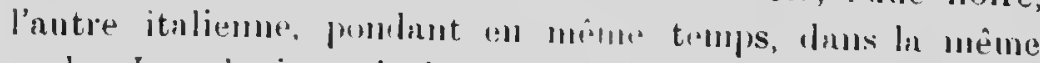

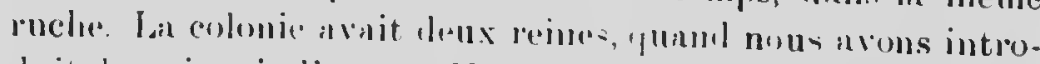
duit la reine italiemu. Sous avions trouvé et létruit la jeune et, sans le salvoir, hai-sé sa mire, que les aheilles estimaicnt le si pun de ralenr qu'elles accepterent notre reine italienne et les laisièrent virre l'une pres de l'antre. A notre graml étonnemrent, il y arnit les abeilles noires éclosant au milien de nos pures italiemes et ce ne fut que lorspue nous en̂mes trouvé par hasarl la vieille reine moire que nous a vons pu avoir. l'explication de l'énigur.

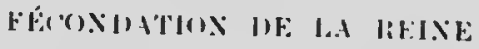

La fécondition de la reine a préencopé pemlant bien lomgtemps les savant; et les apiculteurs et a donné lieu à lien des theories. Si un graml mo mbre de mâles sont enfermés chus me loite, ilsémettent une odeur s forte quue

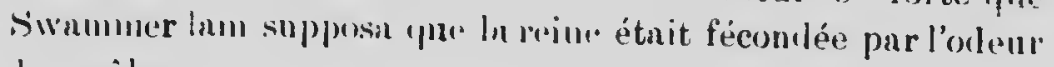
dles inâles. 
Réяnumur, entemologriste renommé, éc isait, en 174t, qn'il pensait que l'aceonplement avait lieu dans la ruche. D'antres

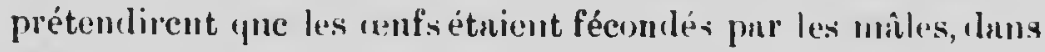
les cellules, uprès qu'ils avaient étí pourlus.

Après aroir finit plnsieurs expériences pour véritier ees diverses théories et les nyant trouvées tontes crrounées, Huber à ln fin, recommut que la reine étnit fícomléx in pleine nir et an rol et pue l'mbluence le ret neeonplemrnt durit plusienrs annees, sinon pendant toute sa vie.

La jeune reine sort it la rencontre d'un wille cing jonrs an plus tôt, après in naisance. Plusieurs apicn'tenrs renommés, tels ine Drier\%on en Allemngne et Neigribour en Angleterre, ont écrit qu'une reine pent sortir a l'âne de trois jonrs. Toutefois, nous n'uvons jamais recomm que le temps écoulé entre la naisanes d'ume reine et si premirire sortio fît de moins de cing jours et nons sommes sur ce point d'aceord arec .l. Alley, netuellement éditra de l'A mericun A piculturist et cert incment le ph monle entier. Lan moyenne est. i élevenr de reines lu lement six ou sept.jours. Qumil l'acemplement est antienpé, c'ent le résnltat des visites

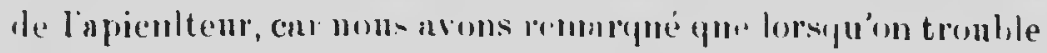
une colomie, ('n lis visitunt, -es jennes ubeilles sont phus vite

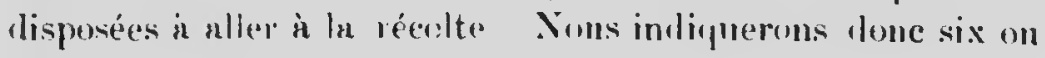
sept jours comme le temps orlinairement necrssaire in me jeune reine arant sa fécurlation.

La sortie muptiale a lieu vers milli: is ce moment-lá jes mâles vo'ent en grmul nombre. Eusortant, ln reine vole la tête tournée dn côté de sin ruclre; elle rentre et ropnrt a plusicurs reprises nvont le s'élnncer linu les airs. Ces précautions sont indispensables pour la mettre à même, ì son retuur, de lien reconmitre son habitation; car si, par erreur, elle se présentait à l'entrée l'me autre ruche, elle y tronvernit la uno:t Cette crreur est une les plus grumles canses des pertes de jennes reines. Si la jenne reine n'n pas réussi d renentrer un mâle dès sa première sortie, elle recommence 


\section{$-3 \mathrm{~S}-$}

It lendermain si lo tomps ast proprice, et le- jumrs suivants sil tot nécessaire, juะqu'à ce qu'clle nit satisfait soll lésir.

Huber nynnt remarqué que le: jonnes reines, nu retour de lenr conrse rnptinle, portaient, sortunt de lenr ablonese, un oljot linonchâtre répuic, recommut hientôt que c'étnit

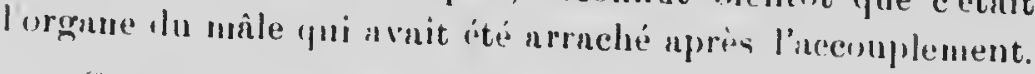

Comme ln rescontre du mâle et de la reine a lieu dans les nirs, peu de personnes ont eu la chance l'en itre témoin. La narntion suivute plairn snus doute in nos lecteurs:

"Il y a quelques jours, par une des rures b.lle- journées que nous r lonnées ce mciș de mni, qu'on s'obstine a "spérer benu et qui est toujours manvais, jerrais a l'aventure dalls les channs non lin de Conrbevoie.

" Tout ì coup un fort hourlonmenent se tit entendre et le vent d'un vol rupile vint pusser contre ma jone. Croyant à l'attaque l'une gruêpe, je fis le ln main un mouvement instinctif pour clıssel l'insecte présumé.

"C'étuient deux insectes dont l'un ponrsuivait l'nutre uvec ncharnement et qui semblaient tomber des lanteurs de l'air. Etfrayés suns donte par mon greste, ils rolevèrent leur. vol et renontèrent presque vorticnlencnt à une trés grande hanteur, toujours se poursuivant.

"Je crus ì une batnille et, curieux de conmaitre lissue d'un combat nussi furieux, je snivis de mon mienx leurs évolutions dans l'air, me prépurnut à suisio les combattants aussitôt qu'ils passeraient ì nu portée.

" Mon attente ne fut pas longne. L'insecte poursuivant s'élevin un peu au-dessus de l'autre et se laiswn tout ì coup tomber sur lui de tout son poils.

"Le chuc dût être violent, car les deux irsectes, qui ne faisaient plus qu'un sroupe, tombierent tout en volant, avec la rapirlité d'une flèche et passèrent assez près le moi ponr que, d'un coup rle mouchoir, je les abnttisse dans l'herbe. 


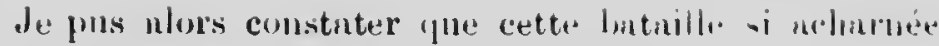
etut tont simplenent une ponrsuite l'nuons.

Jo les ai pris sur le fait, enr les denx insectes complètemrnt étondis et immobiles étaient bien et dûnent neconplés.

"Lincomplenent mait donce en lieu dans l'nir, an moment vi j'urnis r'u l'un des insectes so prépipiter sull l'autre. Le fait s'étrit passé à une hantenr que je puicéraluer à 9 ou 10 serges nu-1lessis du sol.

"Or ces deux insectes étnient une femelle d'abeille commune, parfiatement cnrnctérisée, et mu fuux-bourdon de son espèce.

Croyant aroir tute les paures bîtes, je ne me fis ancun scrupule de les piquer tontes deux sur la même épingie, encore nccouplées at lans leur position respective.

" Ihis la donlenr les imppela ì l'existence et le mâle ne turdu pus à se sépurer d. la femelle.

"La femrlle virnit enen a le temlemnin. Quelque temps après sa séparation d'ave le mâle, elle so lorossait les d.ruiers ammennx do l'nhlomen, comme ponr ex extraire le pénis. Elle fuisait des rfforts pour se plier an deux et sins doute pour monener cettr partie le som corps is poltéte le ses in unlihules

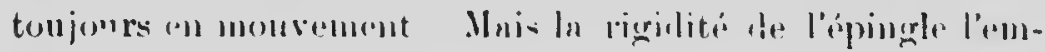
pêcla cintriver à ses tills.

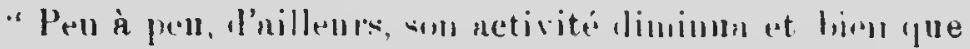

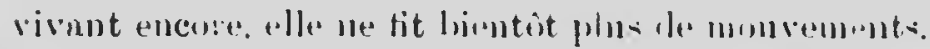

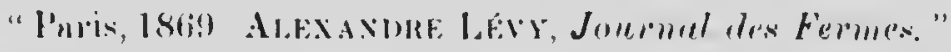

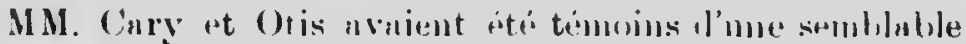

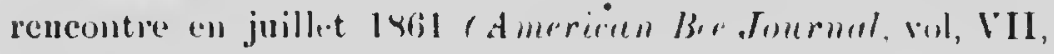
page: 66).

Il ast bien recomnu unjomml'hri ‘n'mu seul necomplement féconle une reine pour tente su vie, quoiqu'il nit até rno snté que parfois des reines ont été fécondées deux jours de suite, leur premier ncoup'ement n'ny'nut pas èté suffisant, notan- 

ment par M. J)evnchelle (Bulletin de la Societé do lo Somme,
1sxis).

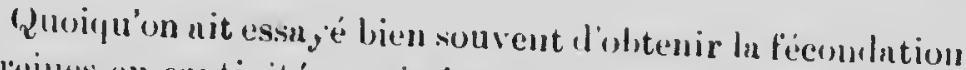

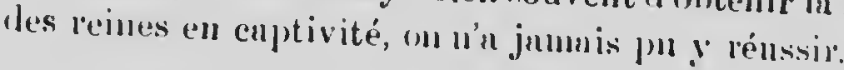

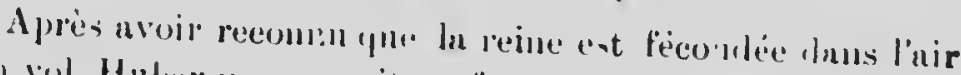

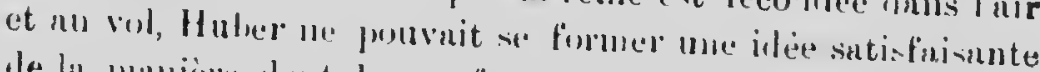
de la maniere dont les aufs sont injuringés par la matière

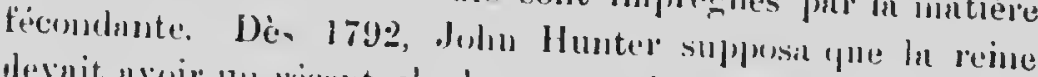

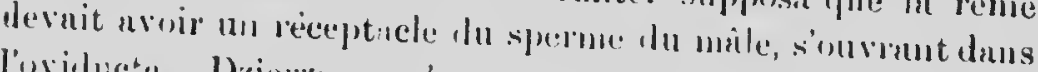

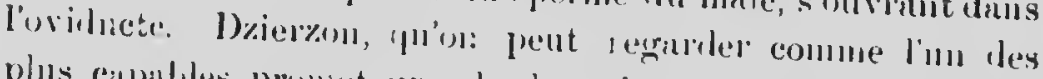

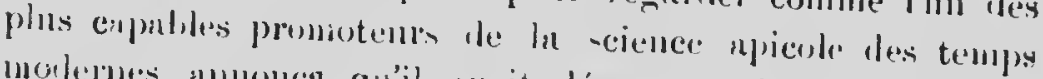

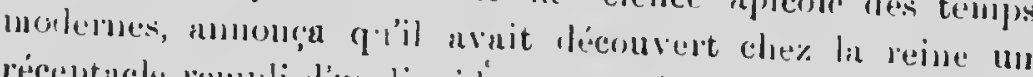

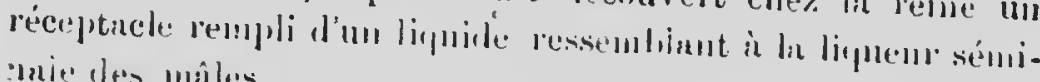
: Inice de's mâles.

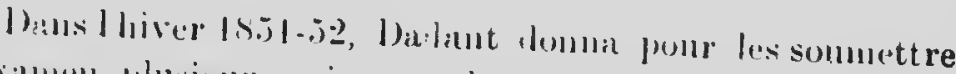

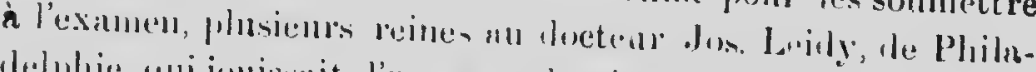

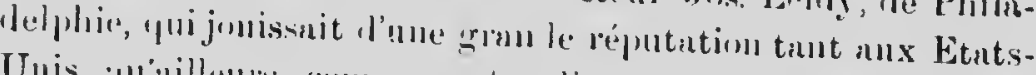
Unis funillews, comme natumaliste et mutemiste micros. copiste. En faisant se's lisvections, il tronsa un sal globulaire d'un millimère de dianetre environ, emmmmignant nee l'oviluete et rempli a'un lipuile blumeluntre.

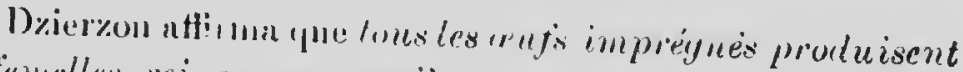

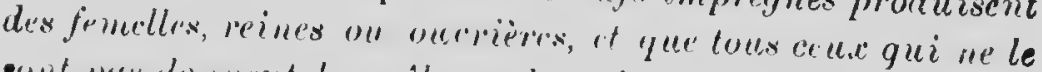

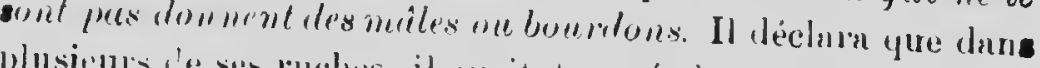
plusicurs de ser rouches, il arait trouré des reines pondeuses de mâtes, dont les niles itainnt of défectneuses qu'elles ne

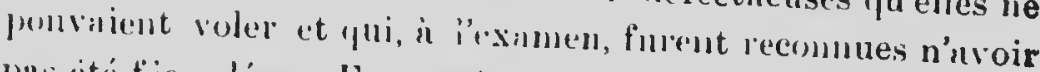
pas itéfiecondées. Ell conséquence il conchnat que les cenfs pondus par une reine non féconclée ont assez le vitulité pour

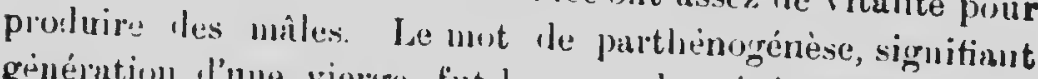
genération d'une vierage, fint le nom donni à cette faculté d'une fenclle de domer missunce à une progeniture sans nvoir éíc fécondée, fuit qui n’est pas lare parmi les insectes. 


\title{
(HAP'TRE: 'TRMISHEVE.
}

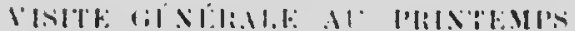

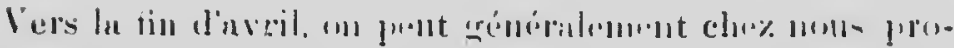
céler ǹ l'inspection des puchen

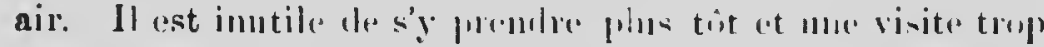

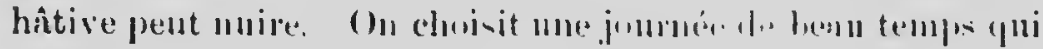

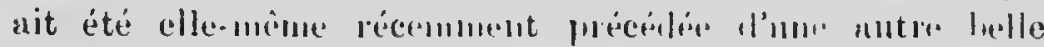

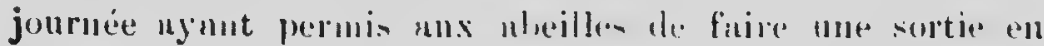

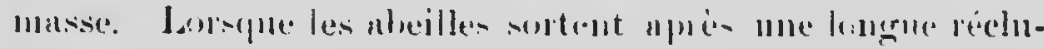

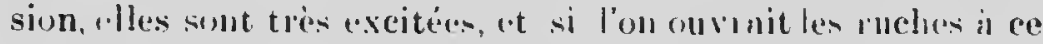

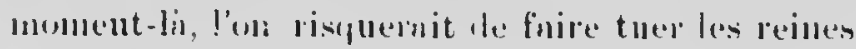

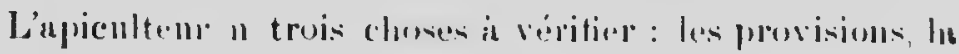
présence de la roine et le colloilin

Provisions. - Fu hiver, tant "fu'il ny n pas ne colvinil, la consommation d'une colonic mise a hivermugr: ans de

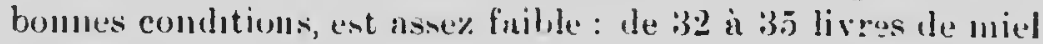
suftisent en génébl pour hien hivernel une honnecolonie;

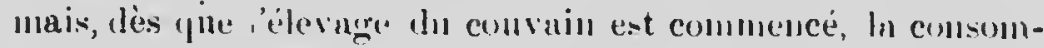

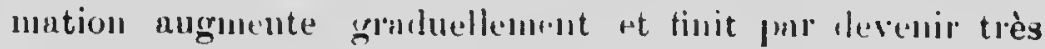
considérable. Sielon que cet élivige a conmencé tiot on tard, ce qui lépend soit de l'étut du tomps, soit de cedni de la colonic, les provisions restantes penvent varier beancoup a la tin d'avril. Il devient lonc necessuire le s'issurel le l'état le ces piovisions.

Pour qu'une colonie pui-se prenlye sou iéveloppentent nurmal an printemps et lommer un remilement, elle loit être dans l'ubondance et toute écomonie que l'upicult"al seruit tenté de firs ale ce ch.f tunlucrait a soll ritriment. C"est absolument comme si l'ugriculteur fnimit l'économie du fumier pour son champ. Or, de la till l'avil it la grande récolte, 
cette coloni: anra hesoin te 12 i $1: 3 \mathrm{~kg}$. an moin", et comme les mirllees qui funvent so presenter dans cette périole: snules, crones, ćruldes, mbres froiticrs, colya, dent-de-lion, mnrrmmiors, stc., sont tries varinlules et tres précnires pour

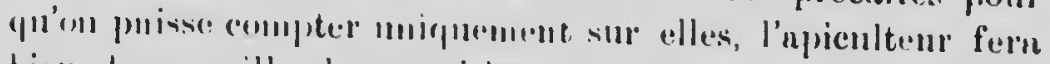

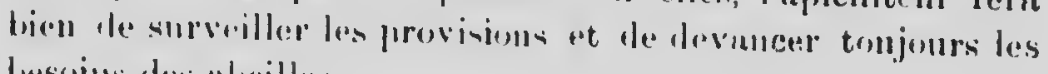
leesoins ders uleilles.

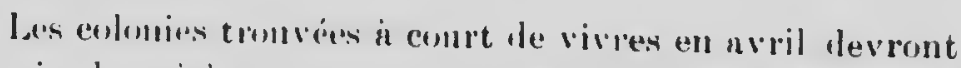

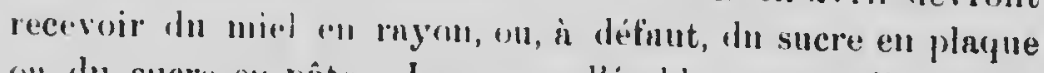
on dn sucre en prite. Ide sucre d'érulile est excellent. Ce n'est que lorsque In tompérnture s'est réclinuffëe, en mai, quon pent dommer do ln nomriture liquile qui excite les uleilles à sortir.

Vin In longueur de lhiver, il fint comme noms l'nvous dit flus lumt, laisser an moment de l'hivernement, nux abeilles nu moins 32 lives de miel.

Recherche de la reine - Pour constater la présence d'une roine, il n'est pas tonjuur's nécessnire ele la voir ; il suffit de s'msurer qu'il y a desceufs. Pour trouver cenx-ci, on sort

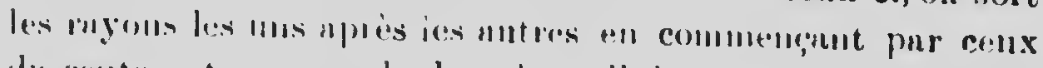

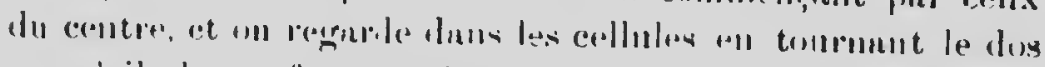

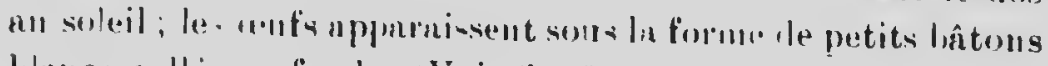
libues colles an foml. (Voir tign 3).

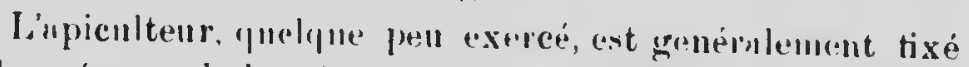

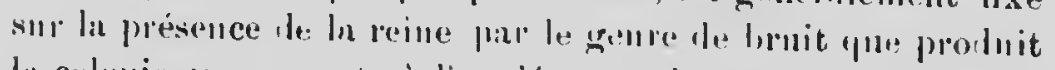

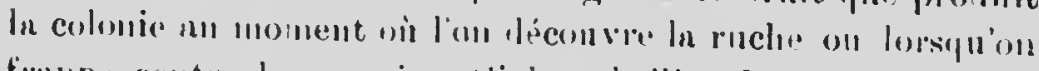
frupp contre les pirosis. si le: nleeilles font entendre un brnis ement vif qui sirrête promptrempnt et frumclument, c'ent que la reine est lin : si ie lirnit se probuge en nugmentunt d'intensité, In colonie est prolubienent orplieline Ce signe est infuillil,e nu printemps lors cles promières visites; plus turd il est moins sîn: II y a l'nutres indices extérienrs de l'nbsence de la reint: Inrsque les abeilles errent devant l'entrée d'un nir inquict ou qu'npıès etre rentrées dans la ruche nve une churge de pollen, elles ressortent anssitiot 


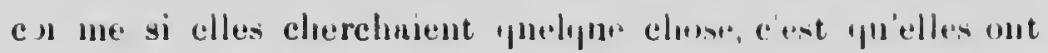

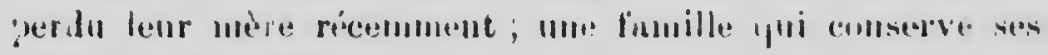

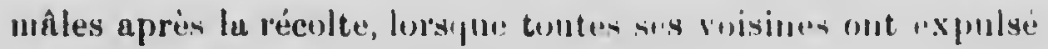
les lenrs, est sonvent wplledine:

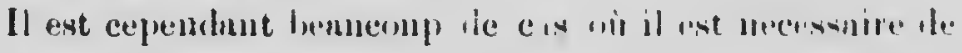

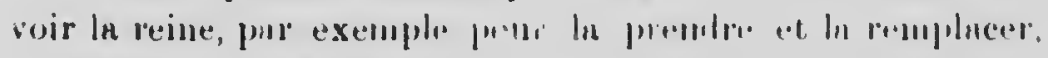

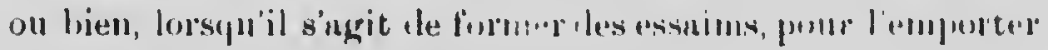

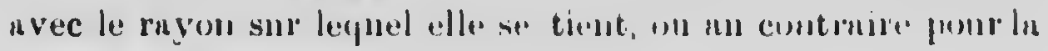
conserver मे lı ruclie.

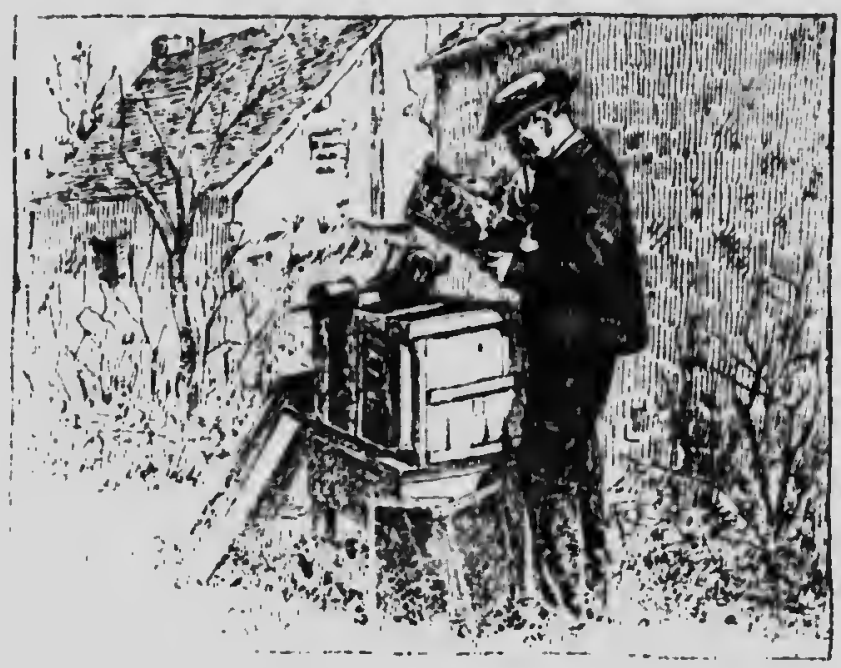

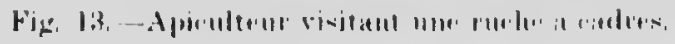

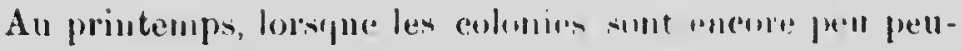
plées, il n'est pus difficile d. In voir. Fille ar tronsw sur les rayons du centre qui contienuent le ronvain, "et en visitnut ceux-ci les premiers, on $u$ ln plns gramle chance des ln trouver

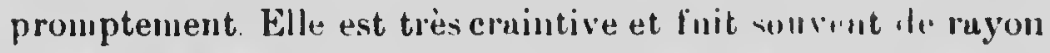

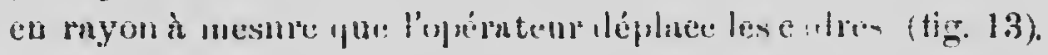

Au contraire, lorspue In population s'est lésaloppée et que la mche regorge d'abeilles, In recherche ite ln reine n'est 


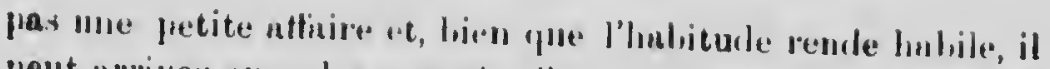

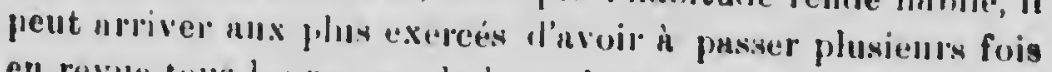

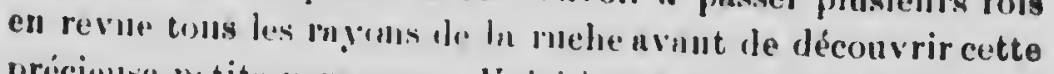
priciense protit. persinne. Voici in ce sujet les lirections que

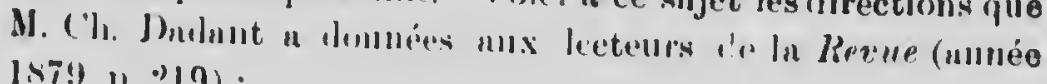
$1 \times 79,1, \div 19)$ :

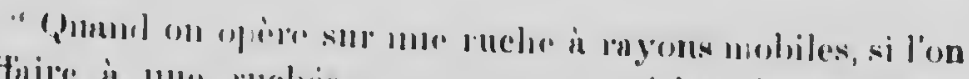

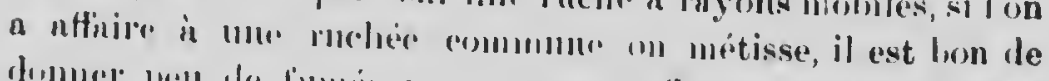

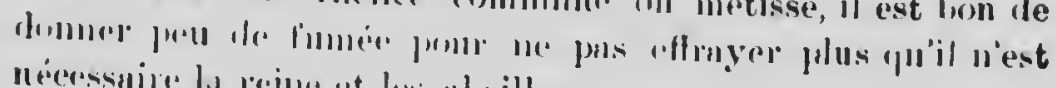

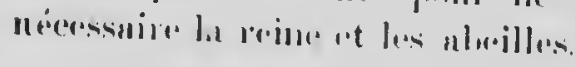

"Ir comsilloria ans diblutunts de linire leurs premiers

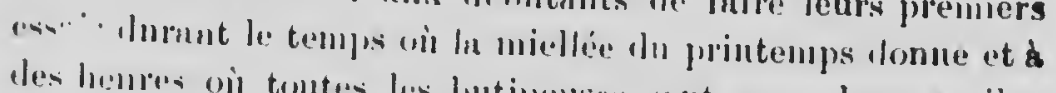

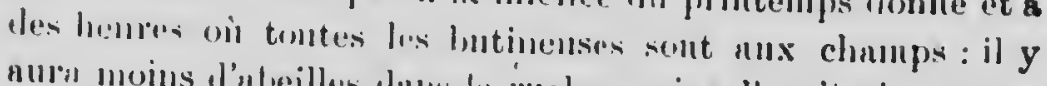

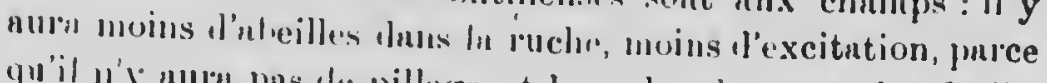
qu'il u' nurn pas le pillage, et In reclierelia sern plus fucile.

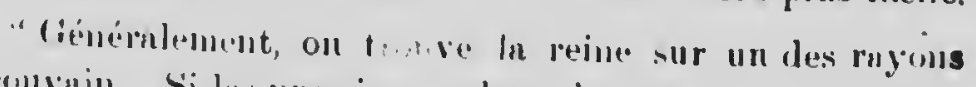
lu convan. Si les premie.s collees n'out pas de convain, on lenr jette un coup l'rril in la hite et on las place en delurs de la phuche le purtition s'il y a de in place dans la ruche,

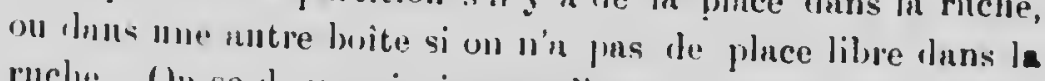
ruchr. (1) se donne ainsi asse\% l'espuce pour pouvolr exa-

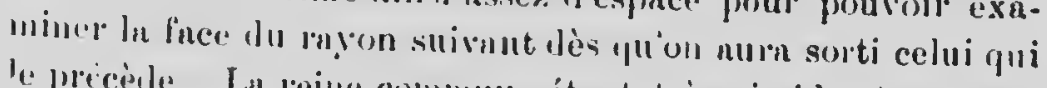
le preciele. La reine commune étant tres timide s'empresse de finire le tour dn layon, stu lequel In lumière fruppe dès quin a leve le précélent, et souvent on pent l'y sinisir ou lever le rayou wrunt qu'rlle l'ait quitté.

si on n'uperyoit pas ln reine stu le ruyon qui est encore duns la I uch", on exmnine nee soin les deux fnces de celui qu'on tient ì la mnin et on passe au suivinnt.

"Si, rprès nvoil visité et exanniné tons les rayous, on n'u pas trouve h reine, oll recommence, en examinnnt les endroits où ln ruine peut se cacher sous les abeilles, ce qu'elle frit souvent quand le temps est frais.

"Si cette nouvelle recherche n'uboutit pas, on sort tous 


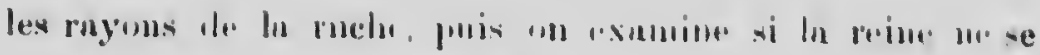

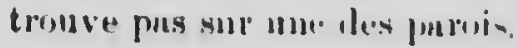

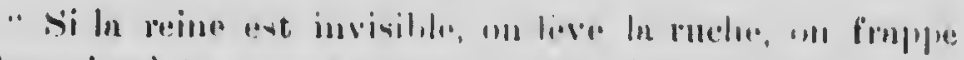

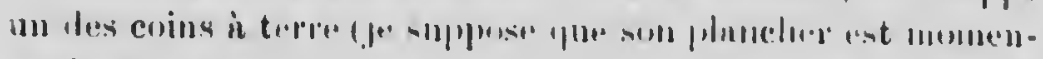

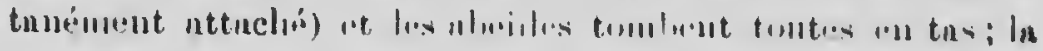

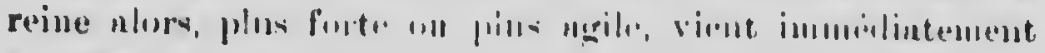

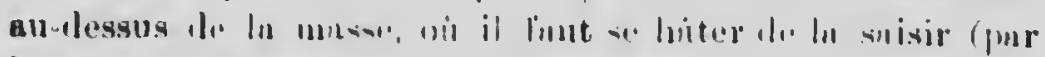

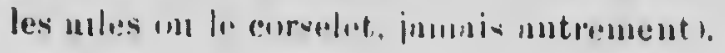

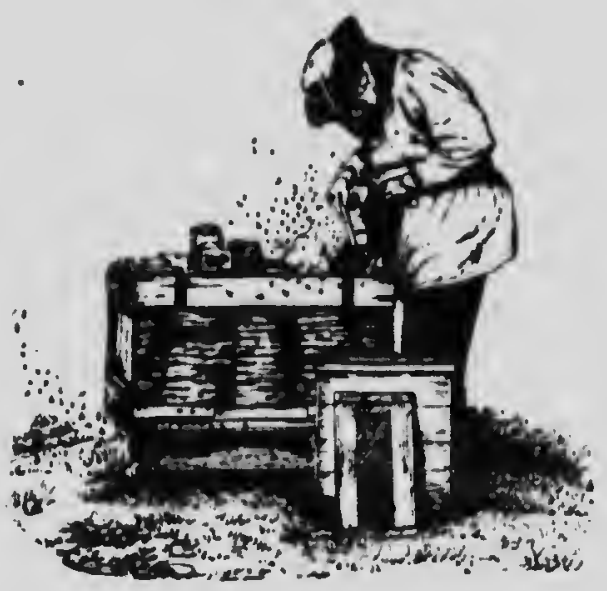

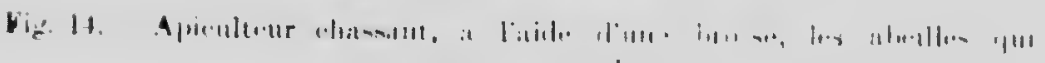

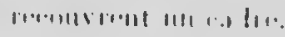

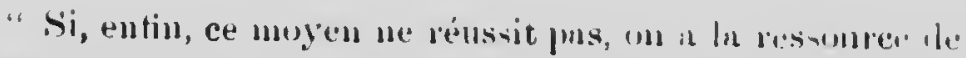

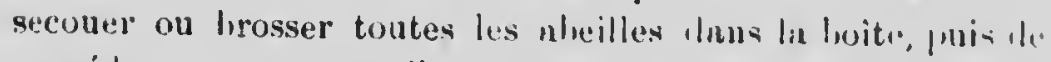
procéler comme nous l'nvons imliqui il la methurle jur le topotement (on verse les ubeilles sur 13 n. trile devant in ruche, celles-ci rentrent an procession et il est fucile de roir et le suisir ln reine, lorspue, escalmulnut les morieses alle se Inîte l'arriver \&̀ la ruclio). (Fïg. 1+l.

En mnyề yui réussit gémérulentent funud ln populntion est forte, mis qui u'est pas experditif, consiste d intercnler 


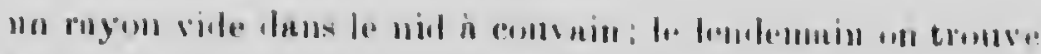

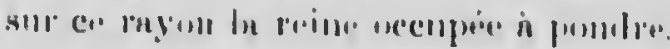

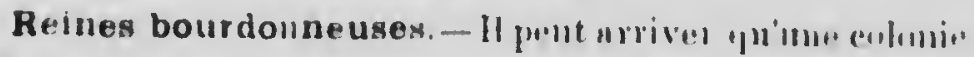

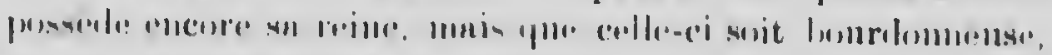

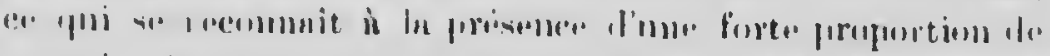

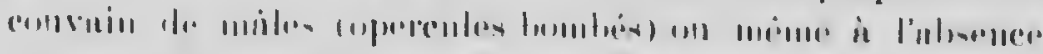

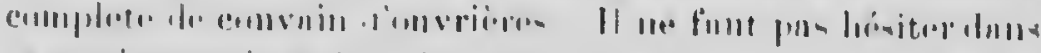

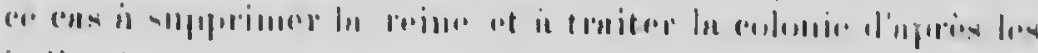
imelimtiums yni rmut -miver.

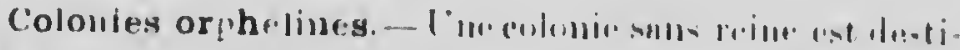

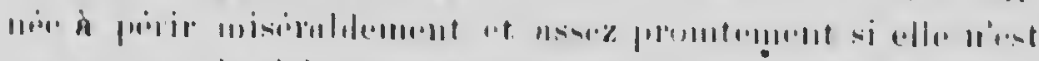
|

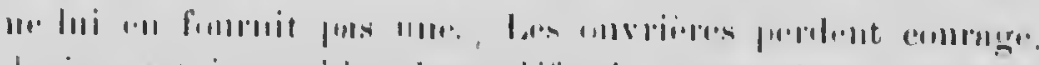

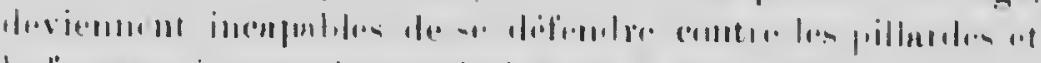

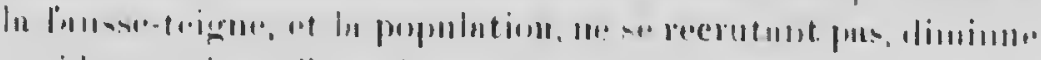

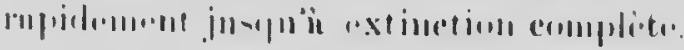

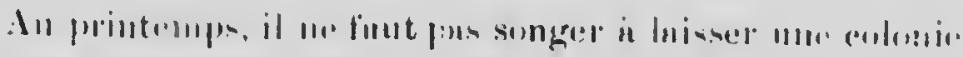

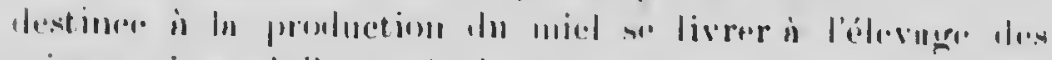

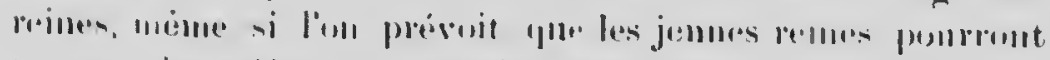

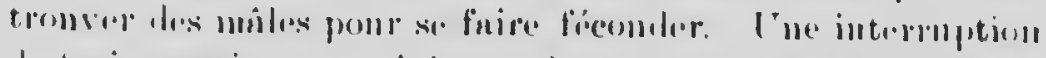

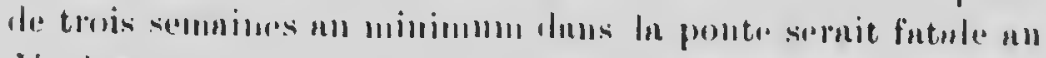

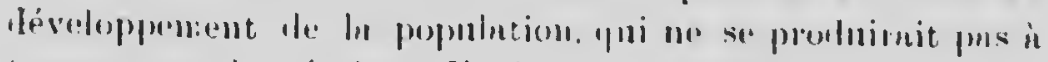

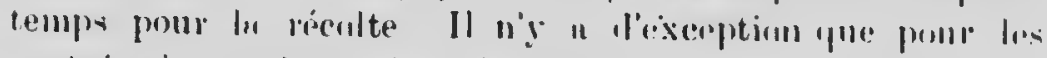

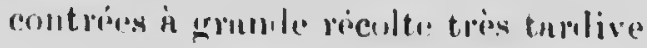

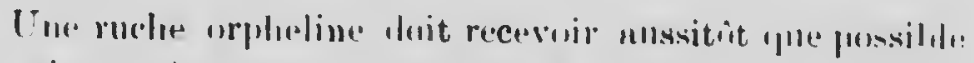

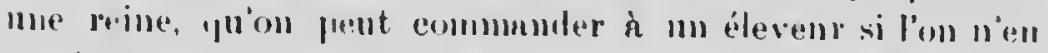

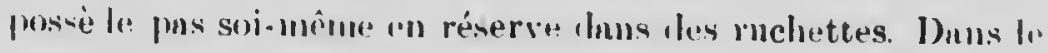
cas ai) l'npiculten ne punrrait pas opérer promptennent ce

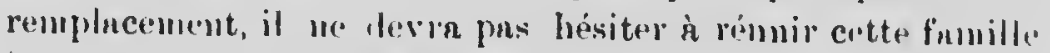
à llur voisinr, qu'elle reuforcern.

Le remplacement des reines ast me opérntiun lélicate, lemanlunt à citre finite uvec benncoup le soin ro fmur Iaquelle on n'n malhewrensement pas encore le rocette infnillible. Il existe mue intinité le procédés l'introrluction : 


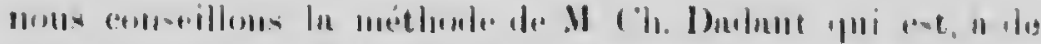

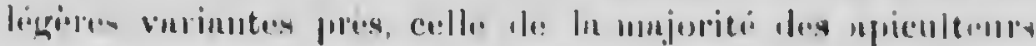

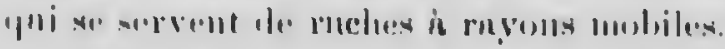

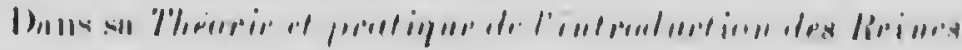

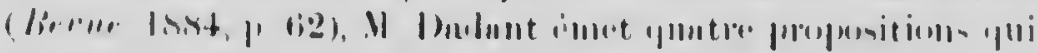

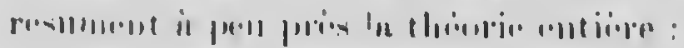

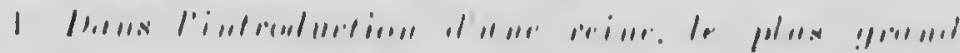

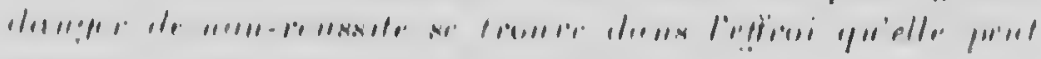

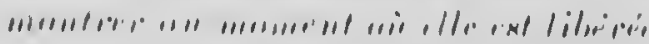

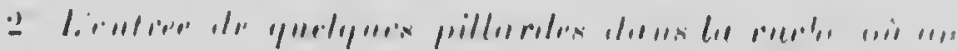

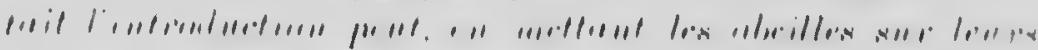

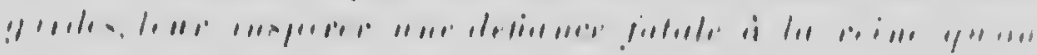

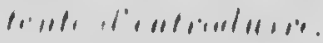

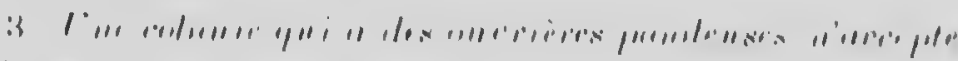

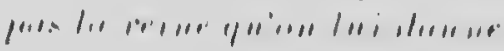

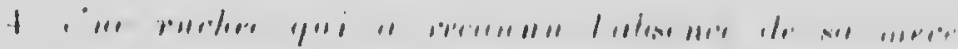

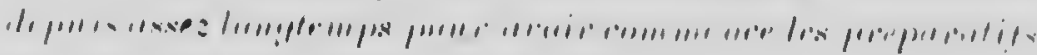

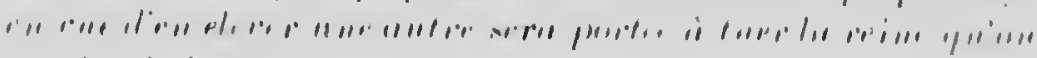

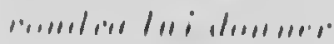

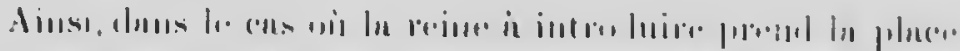

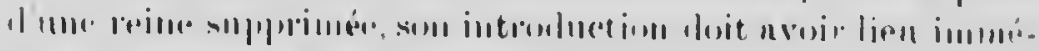

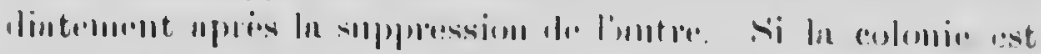

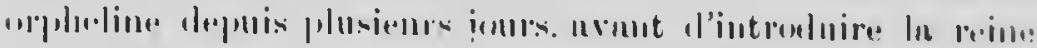

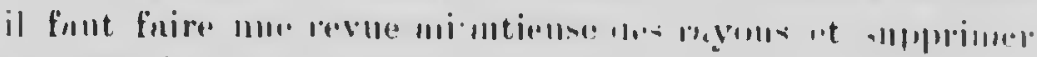

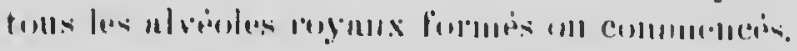

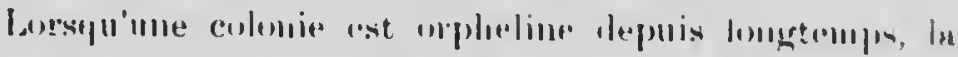

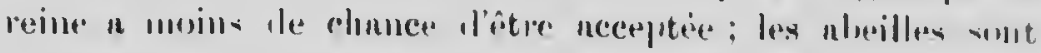

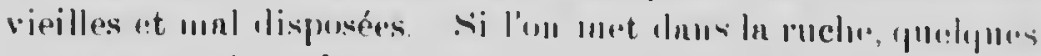

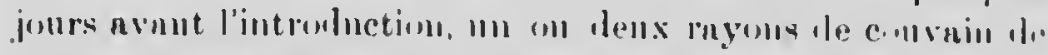

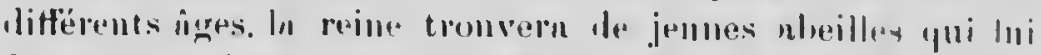

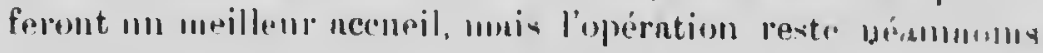

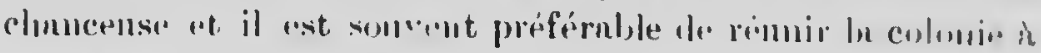
IIIr nutlin.

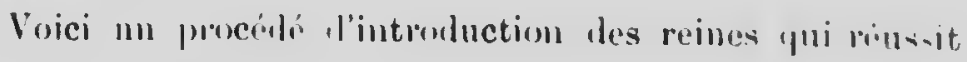




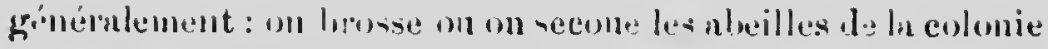
orpheline sur un drap luvant la lonche, on les sampoutre de furine, puis on place an milien l'olles ha reine égalennent enturime. La famille rentre lans la ruches avec la reine,

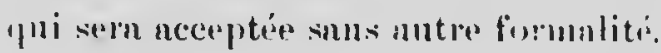

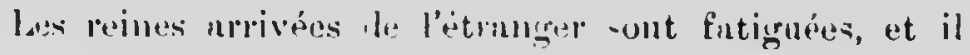

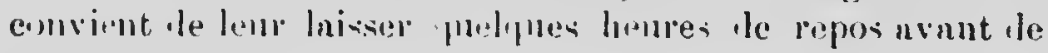
lesintroduine.

Kéu rions - l'onr rínnir denx colmuces ellsemble, opératim rum finit le soir, il fint prénlablement les entumer un pen penr lan finire absurber dn miel et même, pur sureroît le précnution, les asperger l'ean sucrée aromatisée lont on

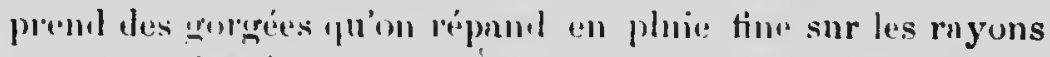
ell serrant les lives. On espace les inyons de ln mehér qui

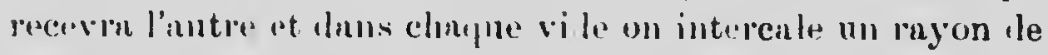
cetle-ci avec nes nheilles. Les nheilles restant daus la ruche

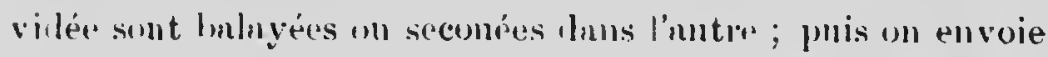

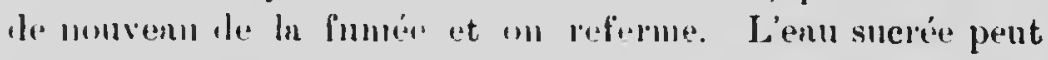

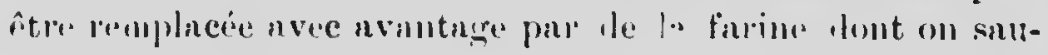

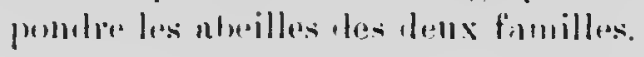

11 fint avoir solu de inomper ensemble nu centre les rayons contenant du comrnin. Si toms les colless ne trouvent pas place dons In ruche, (m) empurte maturellement les moins

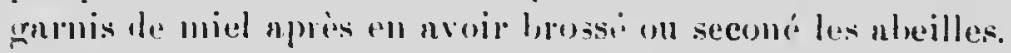

On pent nussi finire mo rémion en secumnt un lonlayant

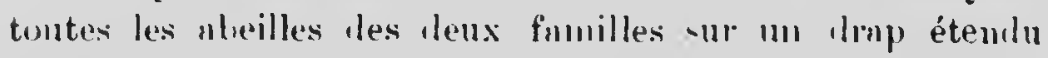
devont la roche et en les sampondrmat de farine an moyen l'm tumis. Elles entreront et se melement snns yn'il y ait

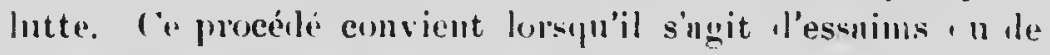

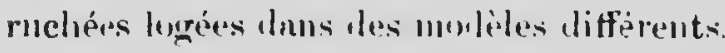

Précautions lors des réunions, déplacements ou suppressions de ruchéer. - La ruche lans lapuelle on vient

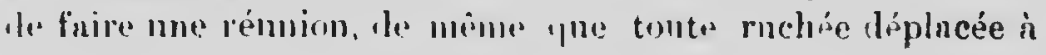

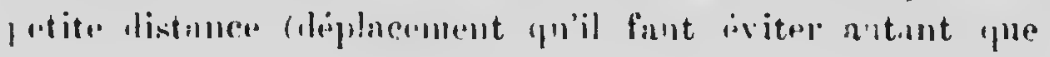


possilh) duit recevoir imms lintement desnnt son entrée une pièce de locis inclinée qui masque suftisnmment le tron-de vol pour forcer les uleilles is s'uperceroir, lies leur sortie, que leur fomicile is chunges, et ì s'orienter de nonvenn uvant de s'élnigner. Au lont de quelyues jours, ln planchette doviont inutile.

Pour déplncer nue colunie ì petite distnner, il est priférable, ci cela se peut, le lui faire fnire nutant l'étnpes qu'e!le a de verges à parcourir; on ln déplnce le soir après chnyue jour de sortie.des abeilles. Il se perl ninsi moins d'abeilles. Antrement on a recours ì l'ohstucle levint l'entrée comme dans les réunions.

si, pour léplucer une colonie, on ln réduit in l'état d'essilin, ell forçant, an moyen de ln funnée, ses aleeilles ì se gr.nger de miel, puis en les secounnt dans une caisie bit elles seront laissées quolques hourea, la famille per I mietix le somenir de son uncien domicile. Ses myons de combin peuvent itre unumentunément contios à une nutre ruchée pui les soignern.

Lorsiguon supprine une colomic par remoion, it funt

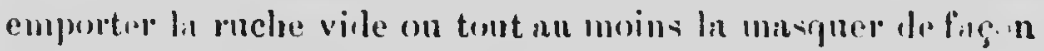

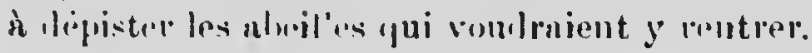

Mais mprinnus notre visite.

Ouvrières pondeuses. - Il se peut entin pau: la ruchíe

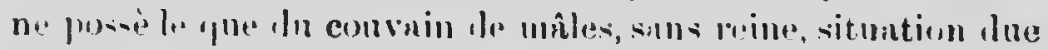

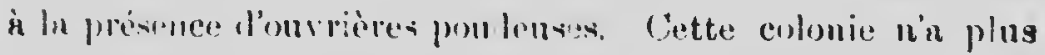
nueume vin'eur et loit êtro lémuntée. Noms emportons la rucho in queh pue listance et secmons les abeilles in torre, apreds leur avir finit aborhy ha miel pour leur Jonner la chance l'ân lim nccueillion par les colonies voisincs. Peut-être randmit-il intunt la létruire. On pent anssi répartir abeilles et rayous entre plusieuri colonies, en prennut les précantions usitére linus les réunions.

Couvain - Las opereules du cournin sont de conleur

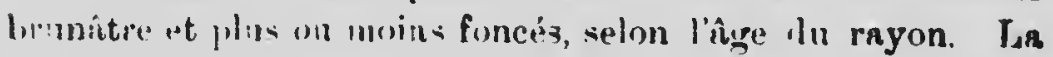


présence de plus on moins grandes plapnes de convain d'ouvrières comprerle est l'inlice d nne lomme reine. Si le couvain

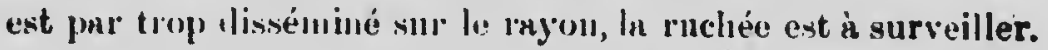
Il se pent que In reins suit nffaiblie, maic on sera micux tixb sur ce point it ln secomle visite huit on dix jours plus turil. (Fig 15).

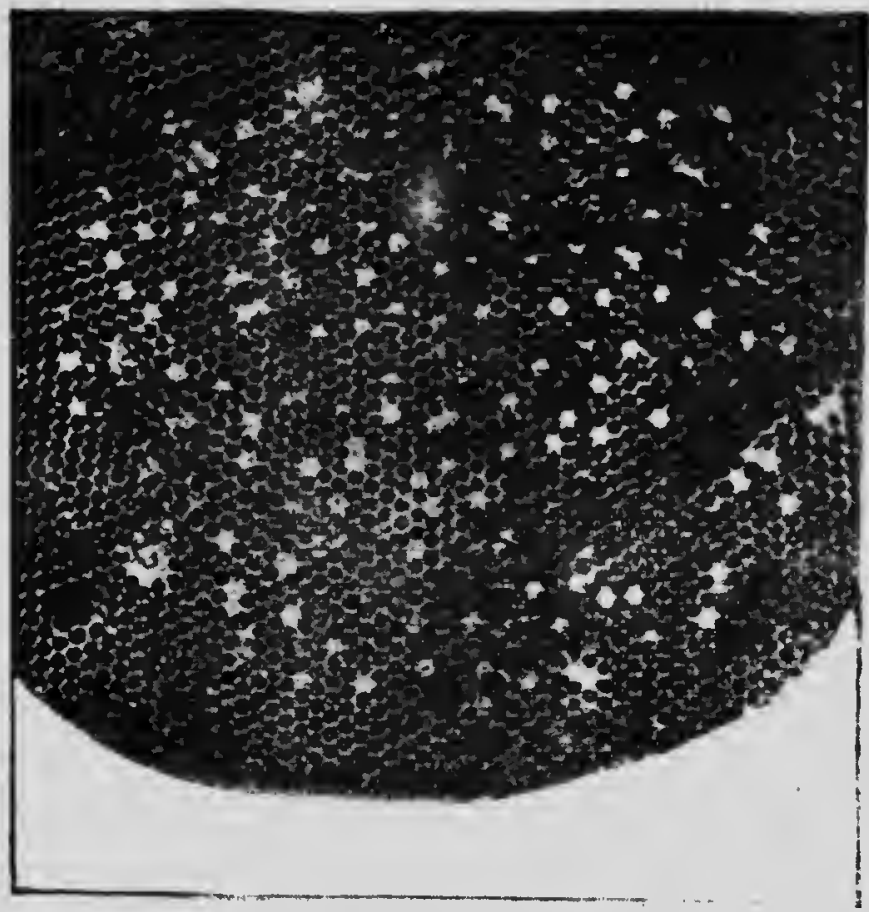

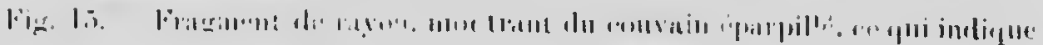

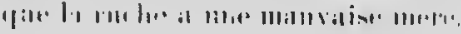

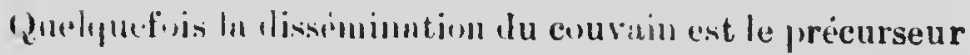

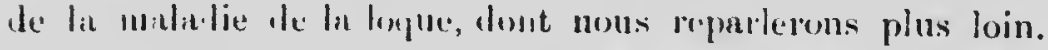
Si l'apicultrum n quépue mot if de se metier de son voisinage

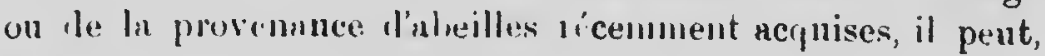

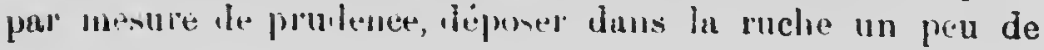


camplire dans un chiffon on du pupier, ou bien un peu do naphtaline et renouveler lu lose nprès évaporntion.

Il peut nrriver qu'une ruche posséfant nne reine r'ait pas encorror conrain à la fin rle la seconde quinzaine d'nvril, bien que le chs soit rare. Te simple fait de ln risite doit provocuer la ponte: si lone clenx ou truis jonrs plus tarl on ne tronve phs lirnfs. c'est que ln reine ne lant rien.

Nettoyage des ruches. - Lors de ln visite, il fuut rncler at essuger les platenn. Le melonir est me lame de

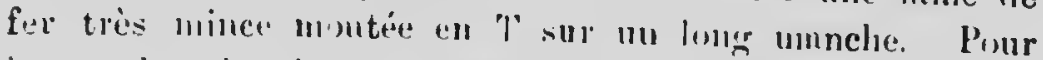

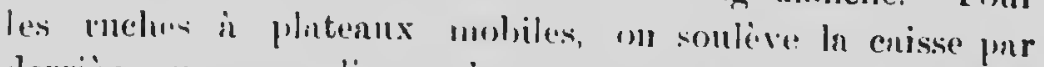

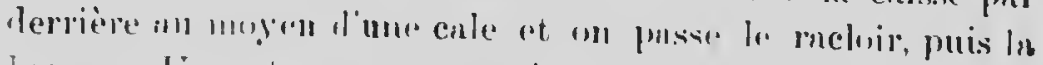

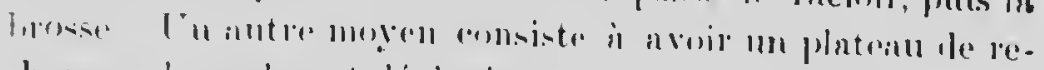

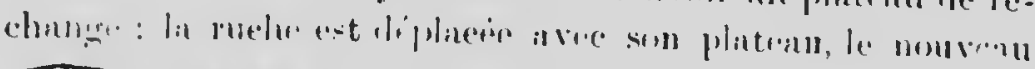

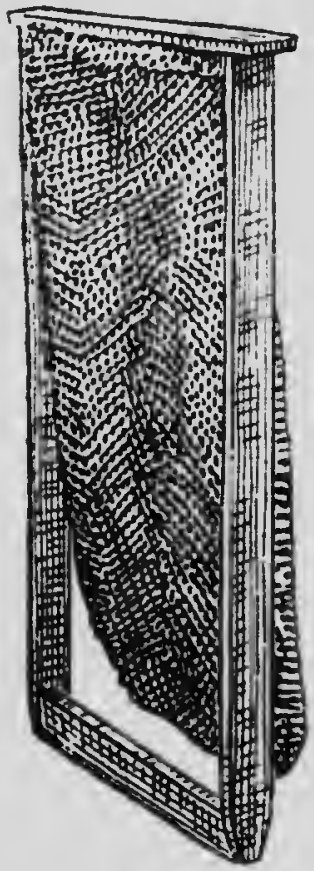

ligig. 1fi. Ratrom mal rome.

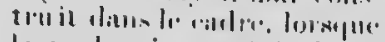

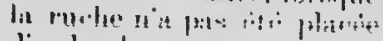
lialululs.

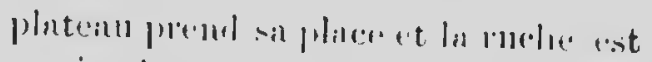

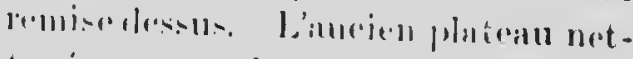

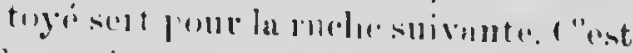

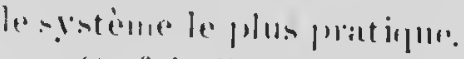

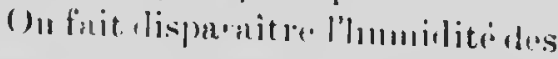
ruches en itiut les convereles yuand

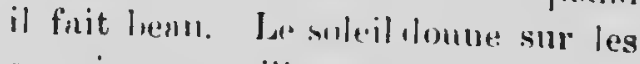
coussins ull prillassulls at oll pompe Thumilite. s'il s'ngit le ruches s'onvrunt pur le coité, on srort les puillussems ot on les expuse an soleil.

Las nheilles wettryent les rnyous moisis, unis si lu pepulation est fuilsp, il funt les iter pour les fuire nettryer phus tarl par quelqua forte colonie ou, ce qui vant mienx si l'on e-t suffisnmment npprovisionné le lnatisses, le.. mettre is lit finta.

lassigu une ruclar ent rlemontée, il

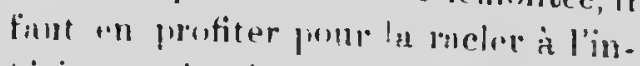

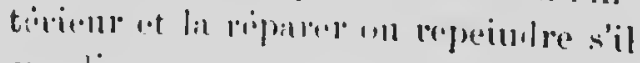
y $\mathbf{n}$ licu. 
A plomb des ruches. - On "l'hubitude, pour l'hiver, d'incliner léruèrensent en avant les ruches mobiles (cela ne peut se finie avec les ruches assemblées en pavillon), atin de faciliter l'écoulement des caux de comlensation. II faut avoir soin au printemps de remettre les caisses bicu d'aplomb; autrement les ubeills : qui suivent une lirection verticale dans leurs constructions, lispueraient ale ne pus bitip lans le plan exact des calles. Linplomb est également nécessaile aı bon fonctionuement iles nonrrisseurs. (Figr 16). 


\title{
CHAPITRE QUATRIEML:
}

\author{
CIRATION HI: YOIYFI.LES CUIONIES
}

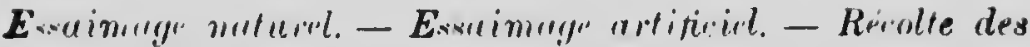

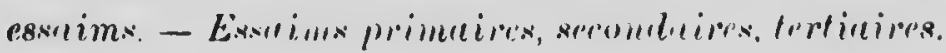

Essaimage. - Dans tont ce qui pricèle, nous avons éturlié l'orgunisition d'une eslonir linheilles et nous arons rn comment som existence est entierement liee à celle de la mère. Si celle-ci menrt et u'n pu êtré remplacée ì temps, ou encore si rlle n'in pas été fécon lie et par suite ne probluit que des finux-lourlons, ln fomille entière est perilue.

Une colonir l'abeilles forme lonc comme in tout complot, comme un senl être vivant qui pent périr tout entier.

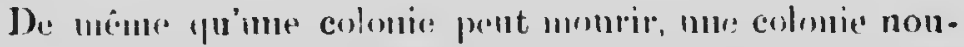
velle pent nuitre, at les sueietis l'aleilles, emstitunnt chacune un orgunisme, se moltiplient et. se propagent comme des indivilus imoles.

Cette mnltiplication des coloniess a rega le nom l'essai-

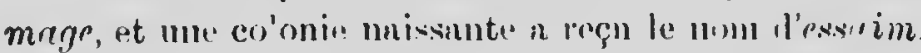

Le plus somvent, c'est vers le commoneentent de l'été qu’il se pro luit un essaim, quanl, par snite de l'necroisuement simultané de la récolte et de la ponte. la rneli: va se trouver trop petite pour In population.

Lorsqu un ruche v' essaimer, il y a tonjours un certain nombre le cellules maternelles en voie le formation. Cing nix sours avant lo trome d'éclosion des plus arnncées de ces cellules inaternelles, la mère sort de la ruche accompagnée 
d'une fraction plus on moins grande te la pepmlation La ruchée pui n dommé l'essaim conserve le reste des abcilles, et, cing on six joms nprès, elle nurn me jemm mire et une seule, les nutres ạ’mat été tuées par cette jemne nière on pur Jes ubeilles.

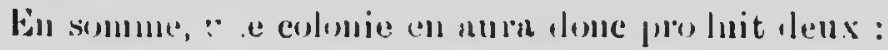

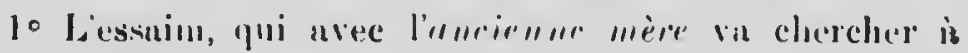
s'étuthlir ailleur:;

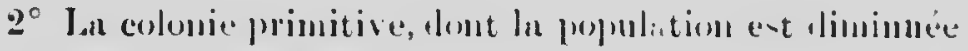

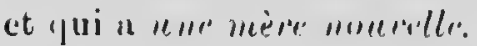

Sortie d'un essaim. - ( 11 n domne un e.ptuin nombre de signes indipnant la prochaine sortie l'un ensim, tel que

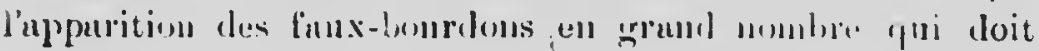
coüncider nver ln production des cellnles de meres, l'excios de population qui déborde de la ruche; rol bien encere le vil-et-vient de monloreuses nbeilles onvrieres qui arrivent de l'intérieur de la rucles sur le platean on inrersenent ; mais

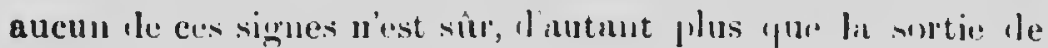

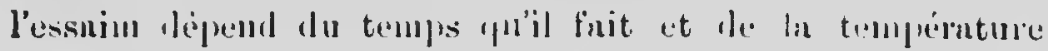
exterienre.

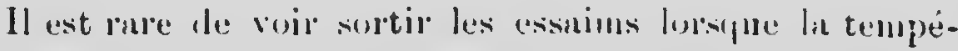

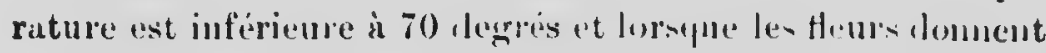

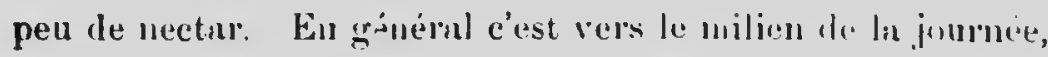

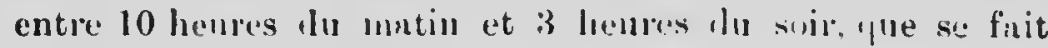
cette sortie les essimims.

La snison de l'assnimigre rarie suivant le elimat tet les plantes mellifieres. Dans mos rigims cent le plus sourent à purtir de la tin de juin.

An moment oì part l'essuim, on roit rapislenent sortir une masse énorme d'alieilles qui tomment autonr de la ruche ou voleut en tout sens en séleviut daus les urs. Mais au bout de très pen de temps, et comme obéissant is un signe de rallieınent, elles vont toutes se rèunir an uncur point, soit our une branche d'arhre, nu-lessuns de larnelle elles se sus- 


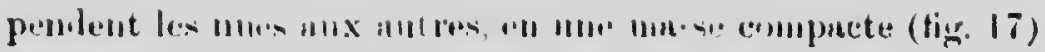

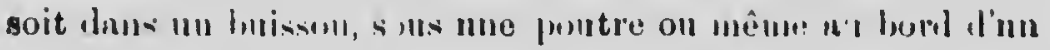

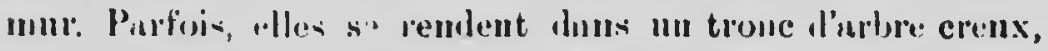
un!: chrominie on tout antre curité d leur convenance. Dans ce Hernier cas, 111 n 1 "I observer des onvrières qui urunt la sortio de l'essaim unt été gì et là chercher aux uleutours un

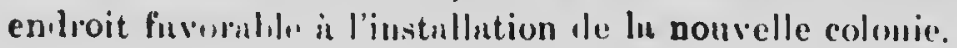

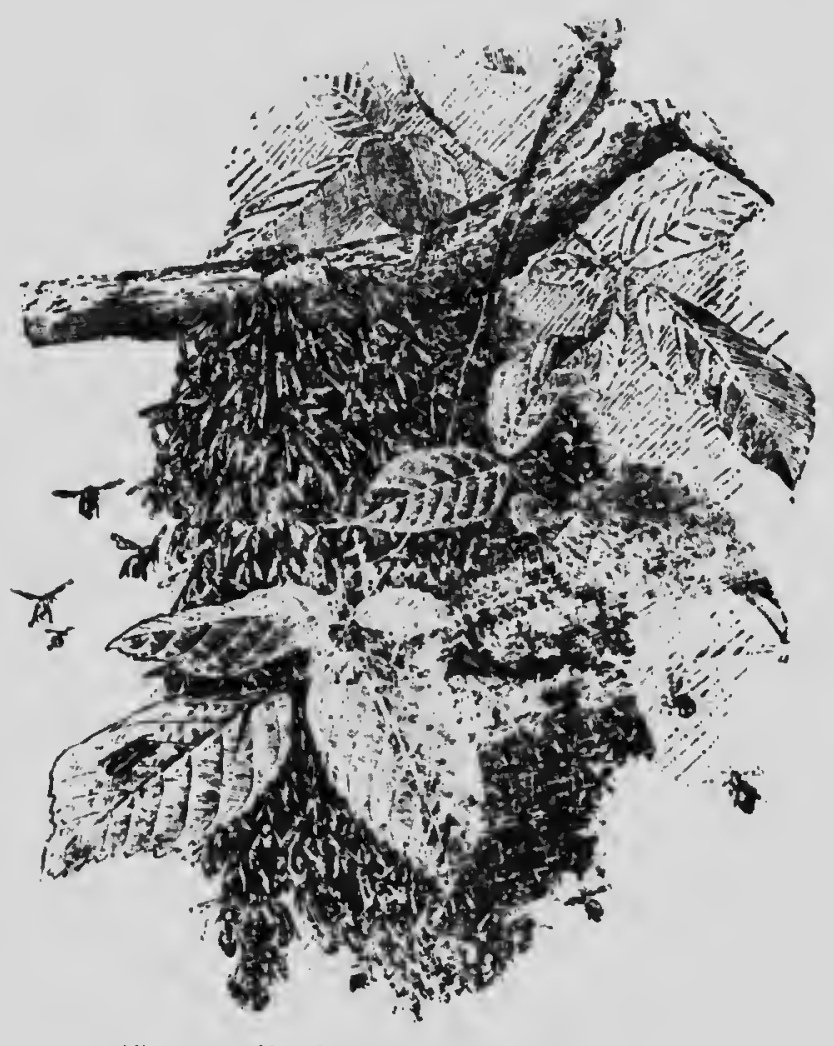

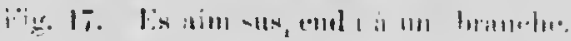

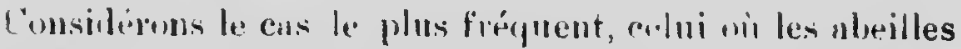

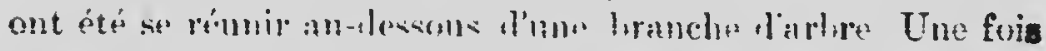
installé sur ce promier support, l'essuim atteml dans cette

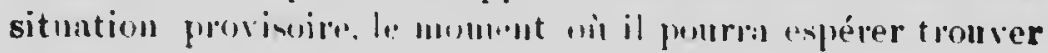




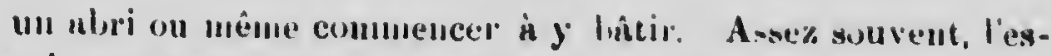
snim ne reste tixé h̀ lu brancle que jusqn'uu lenelennin, [min ropurt ulors pour se poser plus loin, juspu'à ce qu'il uit chroisi uI endroit ensenuhle pour sönstaller définitivonent. II arrive ansis que ne trouvunt menu endruit qui puimse lui courenir, l'essuim continue ì sc léplncor ; il perel de jour en jo'tr des ulveilles, il se reduit de plus en plus rt finit per dispuruitte.

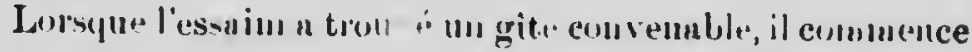
tout le suite fanr comstıbire des rujous; oll pent remarquer d cet éguml que les ousrières qui forment l'essnim sont gurgares de miel et que ln plupart l'entre elles sécrétent ubournmment de la cire. Ia ursivelle colosio sinstulle et levient mue ruche maturelle.

If mrive usie\% souvent mussi qu'un essaim qui vinut de purtio rentre lmus la ruche, soit purce que le temps ent losveun tout ì coup manvais, soit parce que lu mére sest perilue.

Lssaims primaires, secondaires, tertiaires : chant des mères - si la population qui reste cluss la ruclü uprès le départ de l'essniu ast encore suffisante relativensent à la grundeur de la ruclue, il pourra sortir m nouvel essaim,

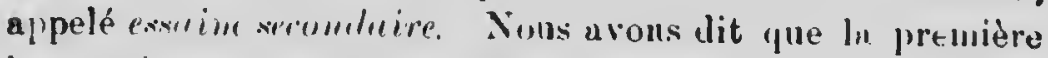
jeme mc̀n ne sort al'mu cellule matemelle que cing ou six jours apres le lépurt le l'essnim primuire. Iolspu'il levra se produire an essaim secomlaim, les mutres mòres enempe dans leurs ecellules me sermut pus tuées, et In joune mère frit

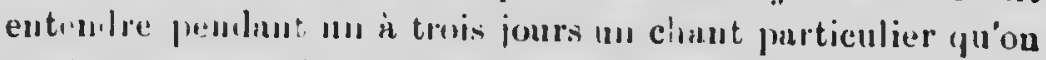
pent representer in peu pres pur til, tik, tile, et qu'on entend facilencent le suir. l.ts mèred qui sunt encore dums leurs

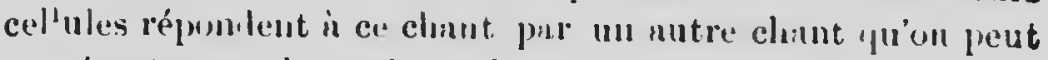
représenter par linere, lienere, loueue.

Ces clumuts spicinux, tries fuciles à recoumaitre, pró-

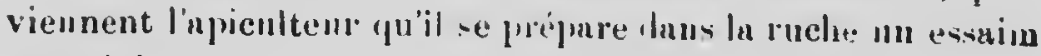
secomilaire. 


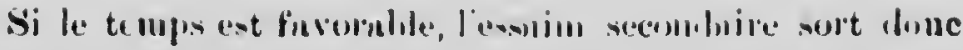

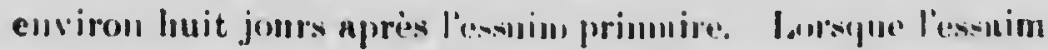

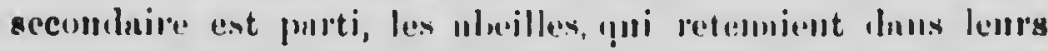

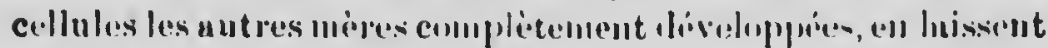
sortir mon, at les nutress sont tries.

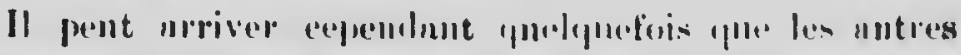

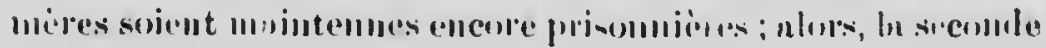

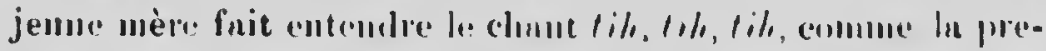

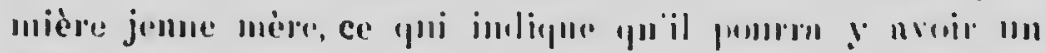

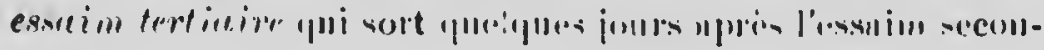
dnire.

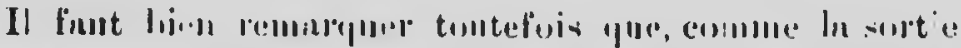

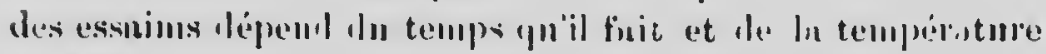

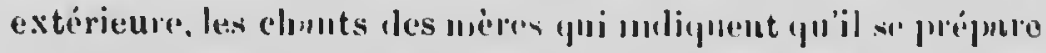
un exsolim secon hipe on tertinime ne sont pus III indice

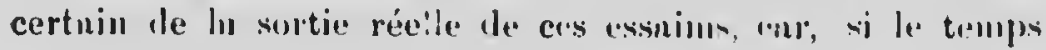

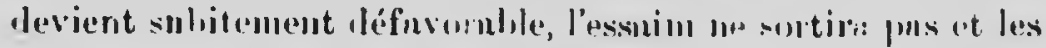
jemnes mères priso : nièress seront tuées.

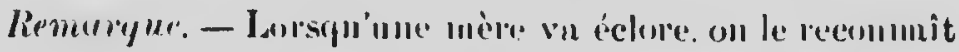

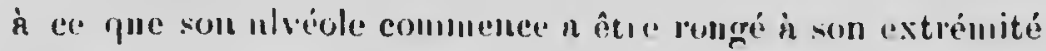

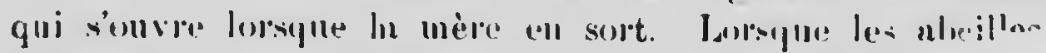

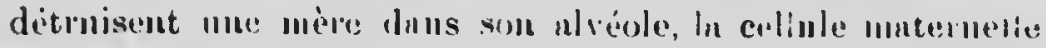
cot onverti: jorl le côté.

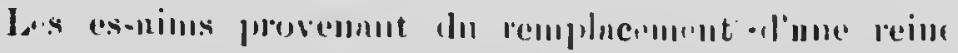

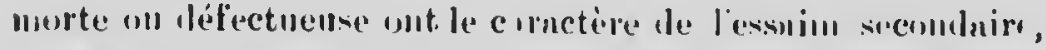
c'est-à-lire que lemr nonvelle reinc at nonvel'ement min at encore vierge, e: ils demun lent las mênes précontions

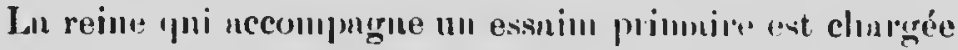
d'ernfs et lourde: anssi l'essnim se pose-t.il tunjon!s assez promptement "piès sa sortie of ne repurt qu'après un temps a sue\% long, qumnd il repurt; tam lis que les essuius secondnires et tertinires, qui ont des reines alertes et viurges repartent plus promptement et inuelquefuis mê ue ne se posent pus dn trout daus le voisinnge : il funt loue se hîtü de les nrrêter et 


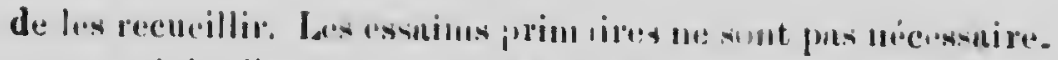

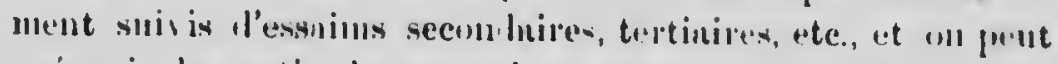

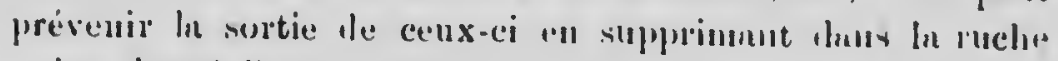

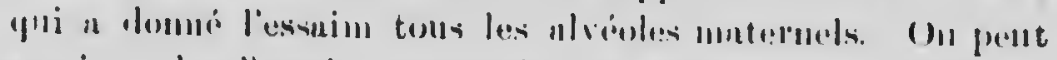

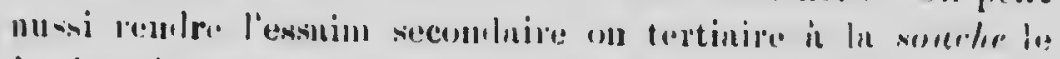

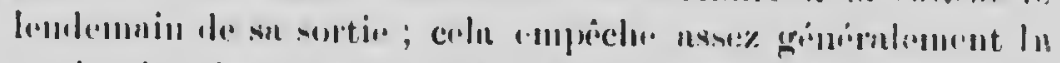

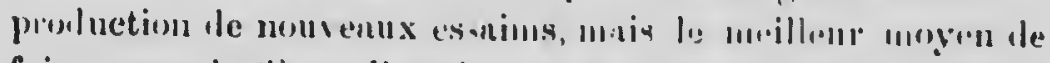

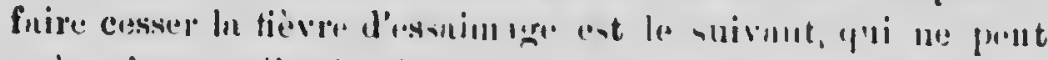

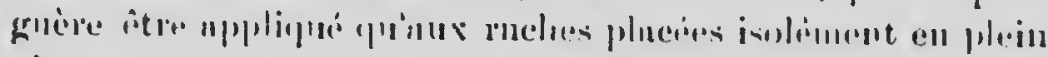
nir.

Prévention dess essaim; secoudaires. - Vinici la

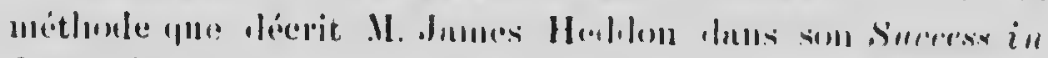

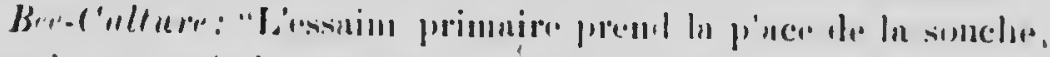

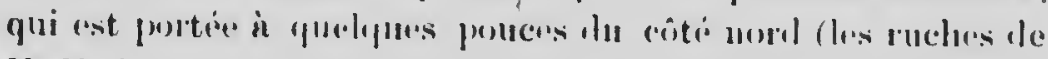

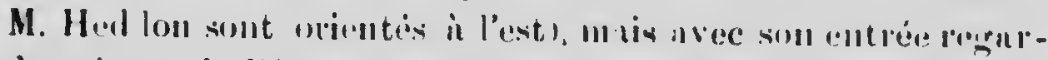

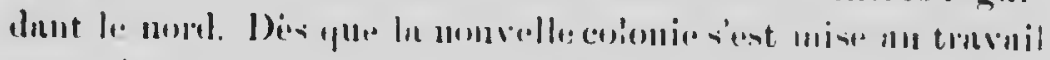

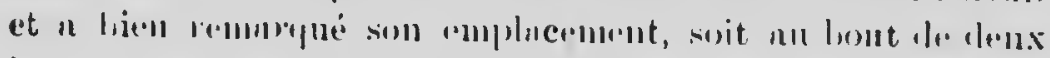

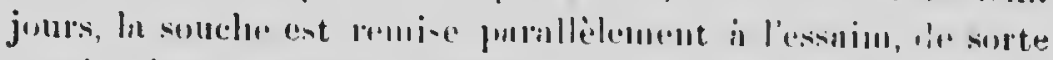

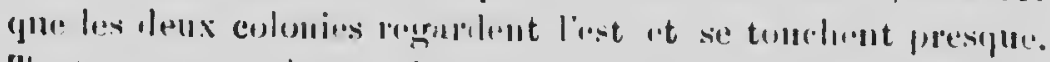

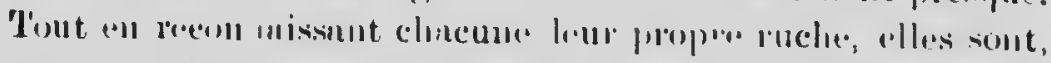

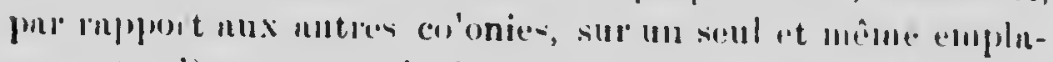

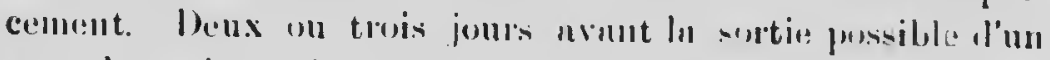

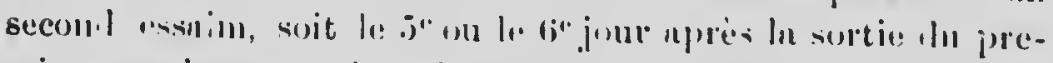

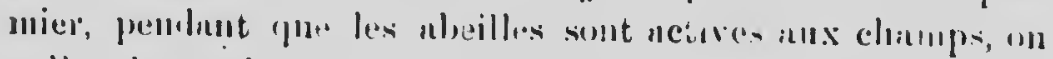
enlive In souche pour la porter ailleurs"

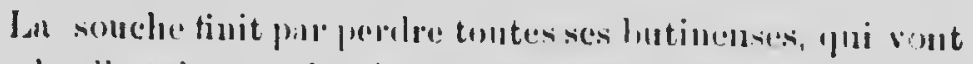

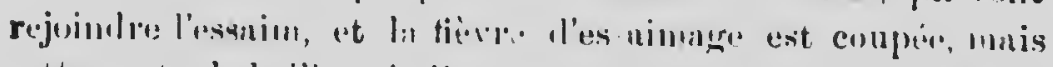

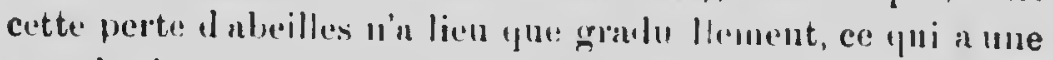
grnnte importunce peur ln sunté dn cournin. Lécéosion

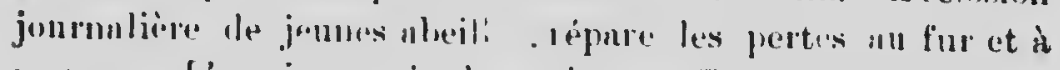

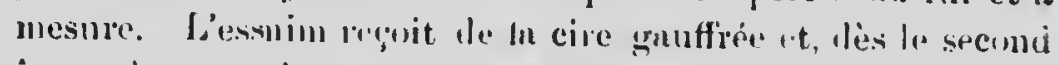

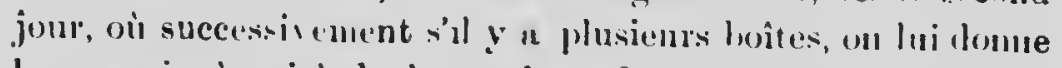

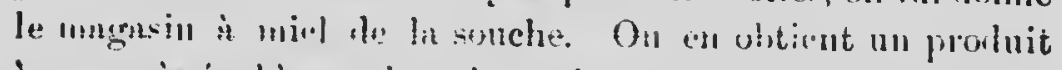
à peu prés érnl ic ce qun'aurnit remblu lu souche si elle ne s'étnit pas livisée. 
Lo procédé Hedilon, qui n'est du reste qu'un perfectionne. ment iles méthodes anciennes, a dunné d'excellents résultuts.

Pour reoueillir un easalin, on se sert d'une ruche en paille ou en toile et de son pluteiu ou l'une fietite cuisse légère avec couvercle da coulisses. Si l'essuim tıurnuje trop longtemps sans se poser, on lance en l'nir duns sa direction de l'eau un, d défaut, de la terie pour simulet la pluie Dans quelynes contrées, dans l'liedre par exemple, un lui enroie un cuup de fusil clangré de plomb très tin '. Il se' prose grinérule-

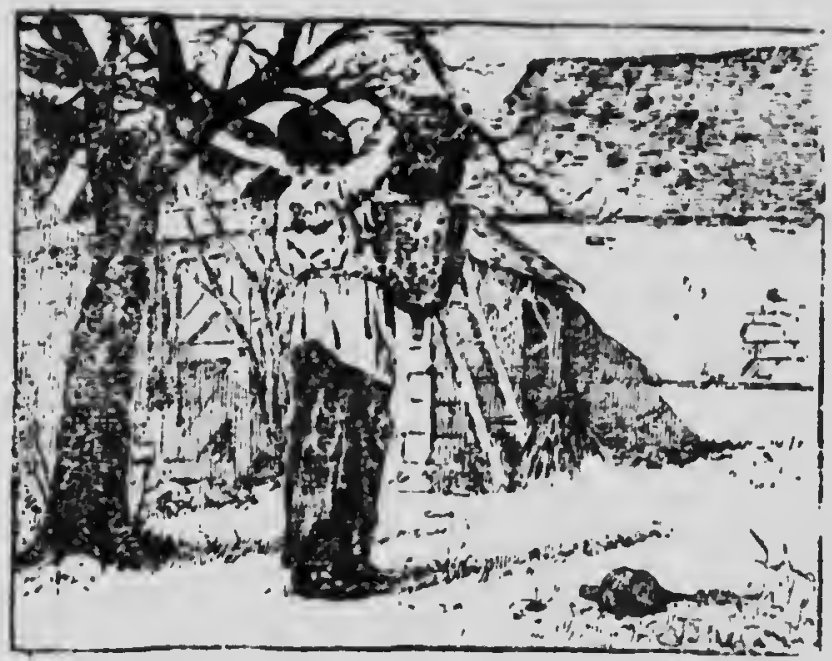

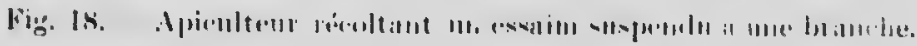

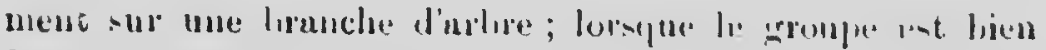
formci, on le fait tomber thus ln ruche en prille on en tuile (ou caisse), on applique le plintean pur-olessus (1n l'on rentre

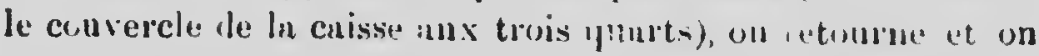
pose le tout h terre, nussi pies que possilile de l'rudroit on était l'essaim, (figr 1s). Si celai-ci étnit puat tnis hant, on

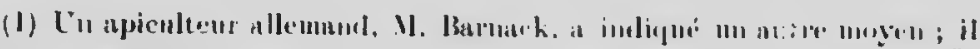

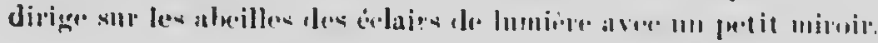




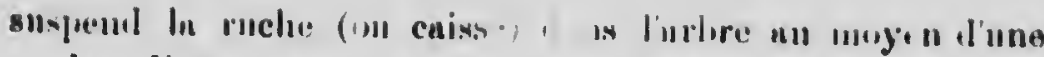

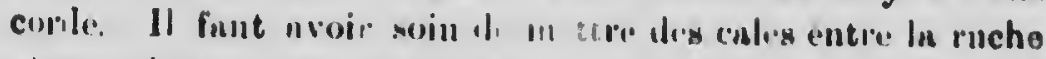

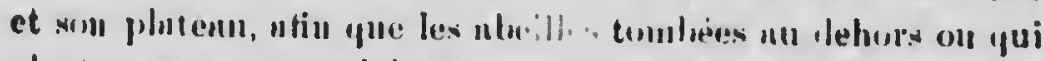

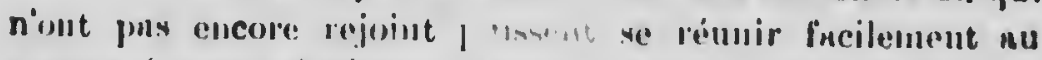

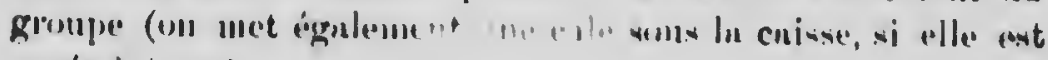
posion is terres).

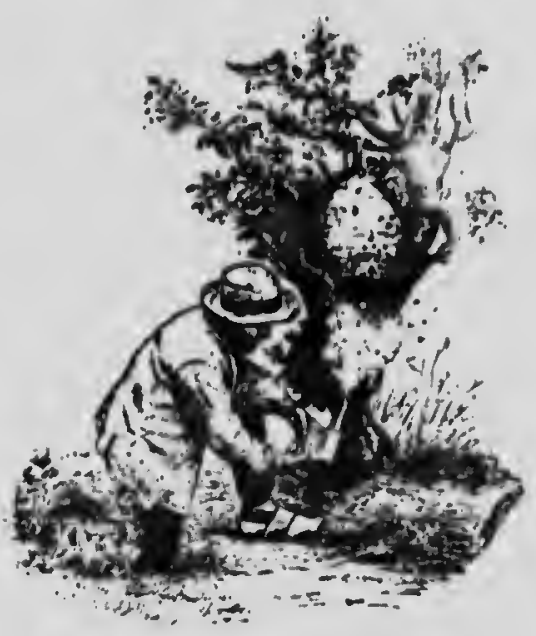

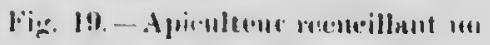

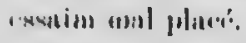

si I rssuin so torlste forle 111 prede le terre, on

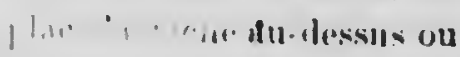
les nbirilles s'y .

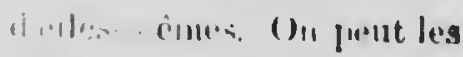

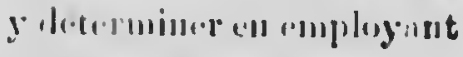

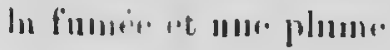

(Fing. 19)

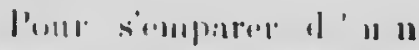

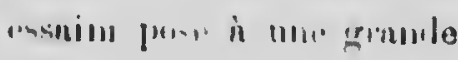
lonnteure, i l'our ne prout

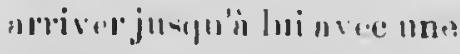
échrlle, (1) amplosion 11

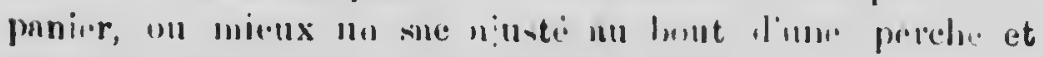

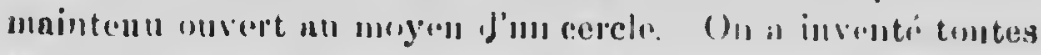

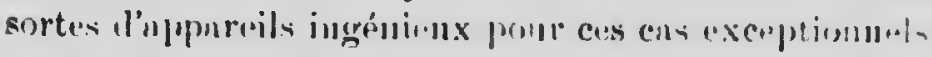

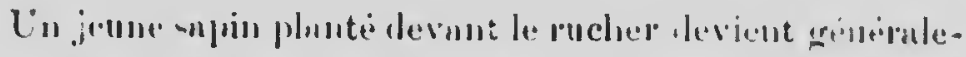

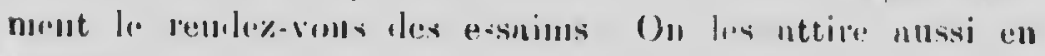

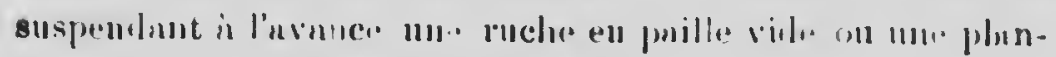

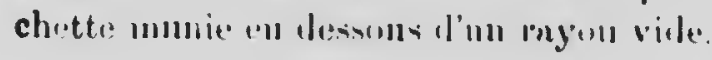

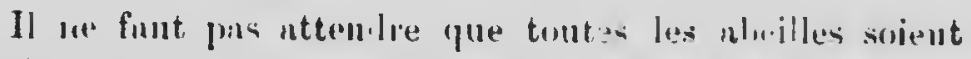

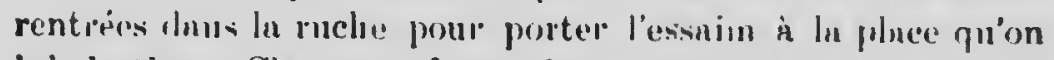

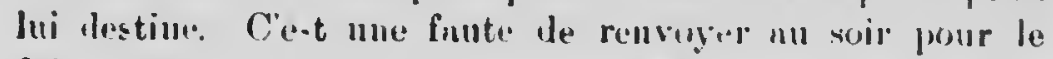

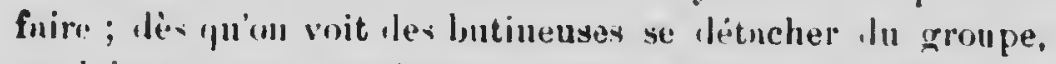

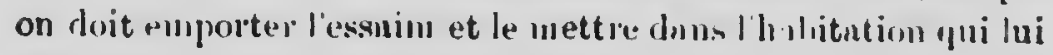
est destinee, on linutıeposer uluns un licul foris et olscur 


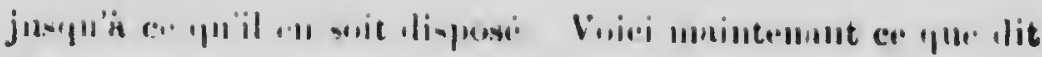

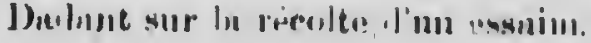

la diblutant in

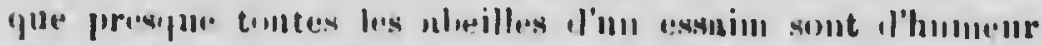

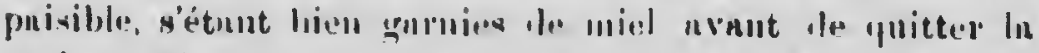
ruche. S'il est fenreux, "II si los piefures le font lwnucoup nouffir, il ilevern se munir llun voile mont de réculter

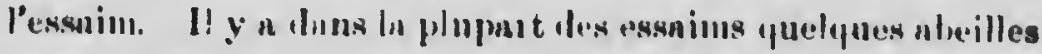
vonunt il'uatres nuchos, un qui, avant de partir, nimt pus pris le soin te se remplir l'outomnc; ces mheilles sont pilus

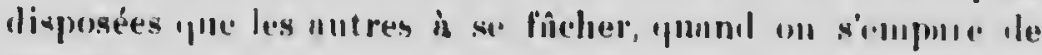
l'ensnion.

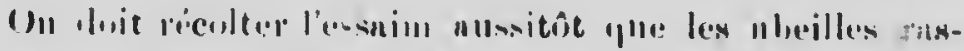

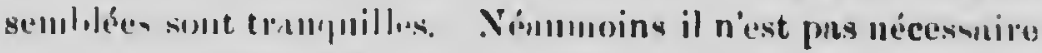

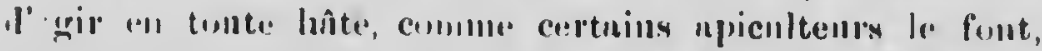

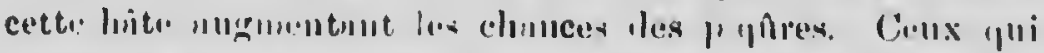

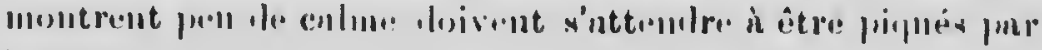

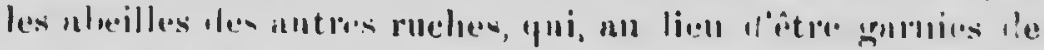

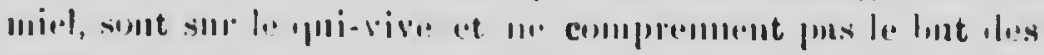

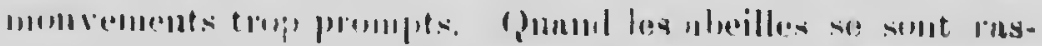

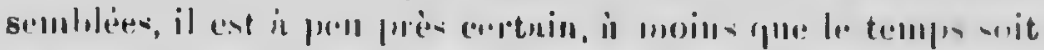

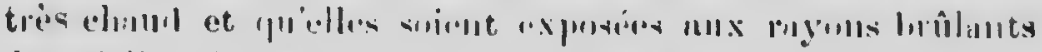

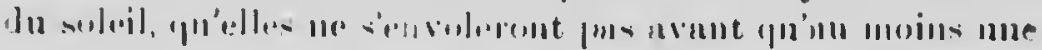

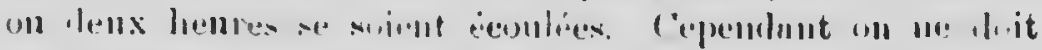

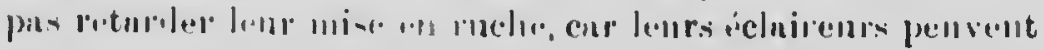

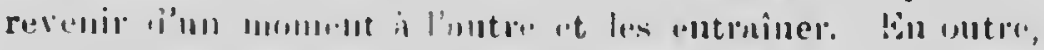

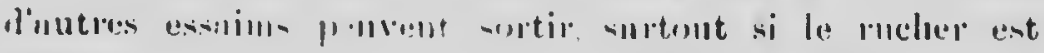

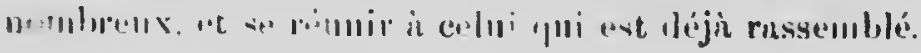

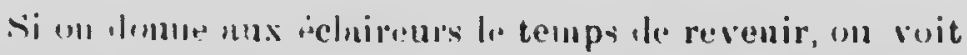

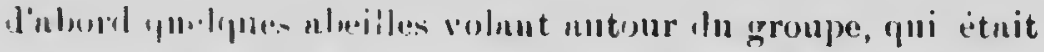
immonlile jusfür co mmment. Petit is petit, le nomlere les

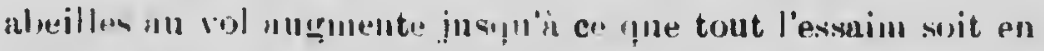
monvemunt. Il semit ulors prest]ue inntile f'essuyer le

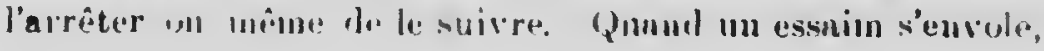


i) ne connait pas d'oustneles. Les luies, les inurs, les fossés, les bois ne sent des barrières que pour son propriétaire essouttlé et désappointé. La seule chose qui réussisse parfois à nrrêter un essain, c'est un jet d'eau lancé en pluie sur les abeilles.

L'apicultrur duit novir une ruche vile et fraiche pour recevoir l'essaim. Quind les abeilles essniment, elles sont très échnuftées et refusent d'entier duns une ruche qu'on aurnit hissée exposée nu soleil, ou tout nu moins, elles n'en prennent possession que lentement. Ln tempérnture le la rucle mère, nu moment dn déphrt le l'essuim, s'élève rnpir'ement et partois à un tel lagré que des nbeilles monillées de:

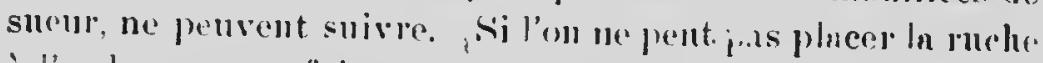
à l'omber pour y faire entrer les alueilles, un doit. in eomrir

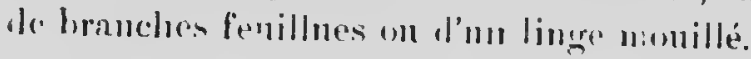

Qnamel on emploie ln ruche it myone molites, on aleit

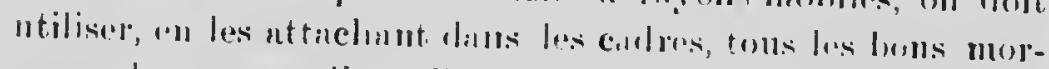

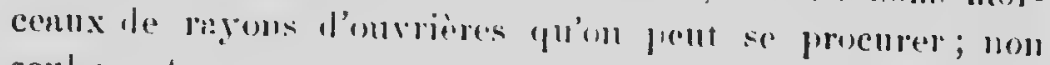

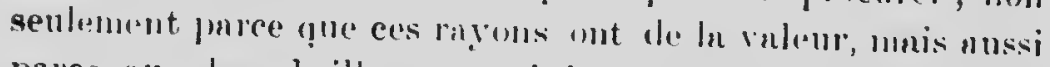
parce que les ubrillus sont si heurues de rencontrer de pareils trésil's qu'elles nlmulumnent rarment la ruche. Des essuims ve logent souvent lans le vieilles rucles nyont des bâtisses et n'entrent que très rarenent dinıs des ruclees vidles, quand il y en nurnit des louznines à liur portée.

Un inconvénient que présentent les rayons domess ix un essaim, ’̀ moins qu'on en remplisse entièrement h ruche ou qu'on ne garnisse la place ville arec le la cire ganfrée, c'est que la reine ne pouvant suive en pondant les ourrières qui continurent les hâtisses, celles-ci construiront trop le rayons de mâles.

On ne doit janıis plicer de rnyon: de mâles dans les ruches avant d'y loger un essaim. Il en est tonjours l,âti assez pour l'élevage de ces glnutons inutiles. Les cadres qui contiennent des rayons d'ouvrières venant de colonies 
mortes l'hiver précédent sont excellents, si les ruyours sont secs, proprus et alsolument viltes te miel.

(II ne doit se servir rle rayons contenant dı miel, quel. que petite qu'en suit la qunntité, que si le pillnge n'est nucunement $\dot{x}$ crnindre. Les nleilles refusent-l'entier inns les ruclues oi il $y$ a rles pil!n roles, on si elles y entrent olles en sortent lientoit; si on tient in utiliver ces rnyons, il funt attemlre mu sole on an lendemuin mutin ponr les mettre lans la ruches.

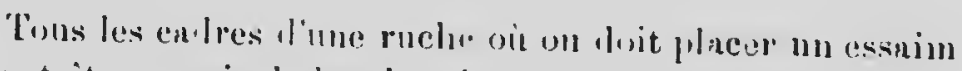

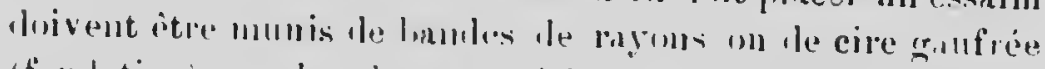

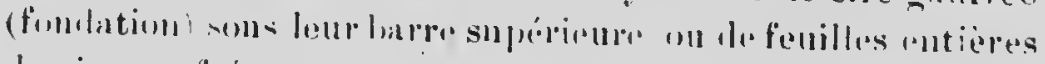
la cire inufrice.

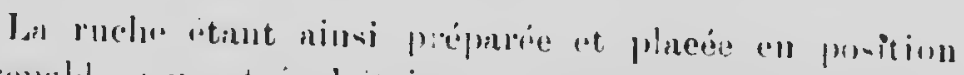

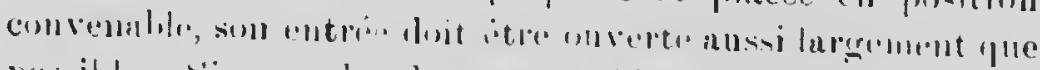

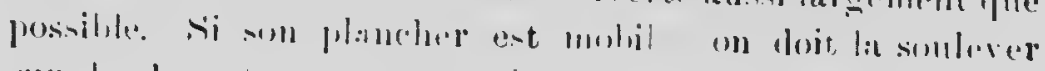

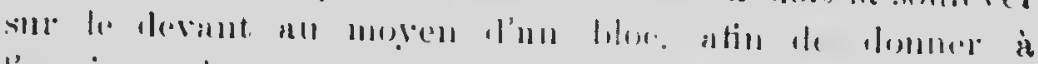

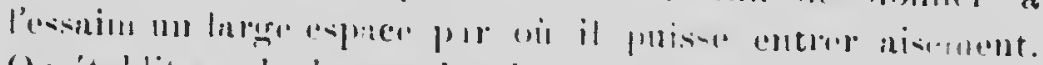

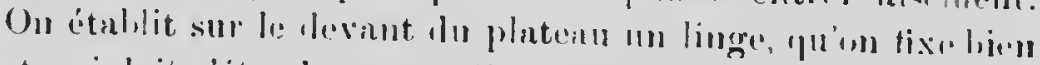

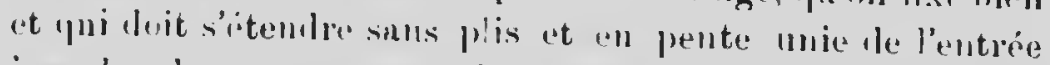

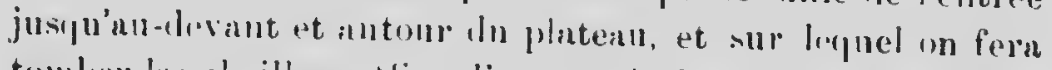
tomler les alwilies. Si ce limere avait des plis sollants, leos ubeilles arotétés par l'obstacle resteraient isolées.

vi les uluilles se sont snspendues a moe petite luanche, qu'ron phise conper ar.c un sricatenr sans la seconer et sans dimimuer In valenr le l'urbre, on peut nisémront la porter sur le linge qui est devant la ruche et l’y déposer avec plécantion. Si les nheilles lésitaient is entrer, on pourrait en lumnser quelques-unes avec une large cuiller ou nvec une tiga feuillue, nu même avec ln main, et les léposer donce. ment tout cuntre l'entrie. Dès que quelques-nnes entreront, elles feront entemlre pir leur luttement d'uiles un son par. ticulier qui communiquera d̀ leurs compagnes liheurense nouvelle de ln lécouverte d'un asile, rt en pende tionps 
lessnim entier sern ims ln rucles sms qu'une scule aleille nit été hlessée.

Qunnd les abeilles.sont sur la toile derant l'entrée, elles ne se soncient pas de repremlle le vol; cur etant nlourdics par le miel, olles désirent. comme des troupes lourdement armées, mather lentrment et posiment in leur place de enmpement.

Quand l'essuim est smspemlu in me brancle élerce quion ne peut atteimlse pour la couper, on quand l'arbie a trop de valeur pom an'on se lécille ì le mutiler, on peut récoltcr les

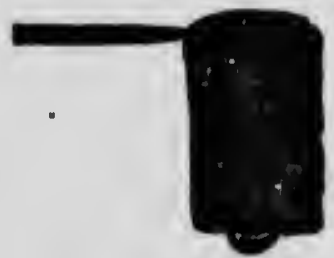

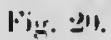

Sil1 il (.)-itill). alieilles ell se servant l'une loîte liggère, ou d'm panier, ou l'un sac d essinim qu'on attache nu bout d'une perche de gramleur suftisante (fig. 20). Ce suc à essuim doit êtro fuit de forte mousseline claire. II loit nroir er. viron 20 pouces de longrueur sur emviron 12 de linuctre. On coud son surerture silr un fort til de ferdont on contines les bouts duns mue petite perche, ce qui le fait ressemblar is mue coiffe à papillons. Si on peut en frire. le fond arourli, cela n'ell randra que mieux. On coud au milien du foml, "ll deflans et en lehors, unc boucle de tresse servant is -oul crer le fonl quanl on veut viler le sac, qui est ninsi, les qu'il est villé et sans yu'il soit nécessuire de le retuurner. pret is receroir un untre essaim. Ponr s'ens servir on met son wurerture tont an-ilessons du groupe d'ulheilles, puis, d'un coup see sur In l,runche, on fuit tomber la unsse lans le suc. II funt sontenir le sne l'une mnin ferme, car le poids yni y tomule est lourl pour la inaiu. En tenant le munche verticalement, me emprelie les abeilles de sortir, l'étoffe fermant l'ourerture.

Il funt premlar qu lques preantions quand on vile le sac. en le retomrmant fe lessus en bas par la trense, cur cet emprisomement imnttemlu : pu fâcher qun.lques uluilles; il 


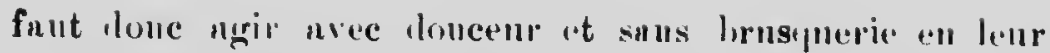
dinnunt ln liherté devant ln ruclie.

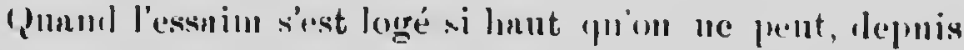

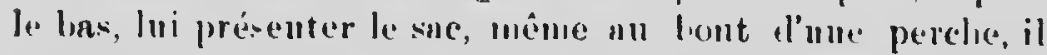
funt monter auprès; puis, tés unc les uheilles ! ont cité

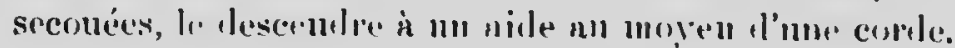

si l'essnim s'est josé in terre, commu celn pent urriver qu.mul lu roine est lomple, ,

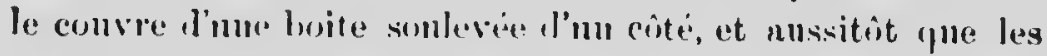

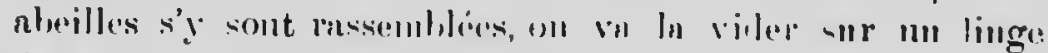
levent In rocles quin lui lestiuce.

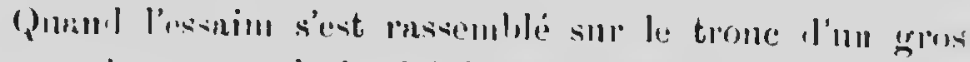

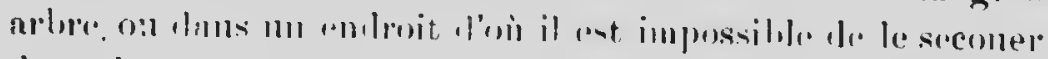

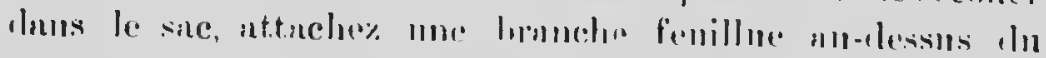

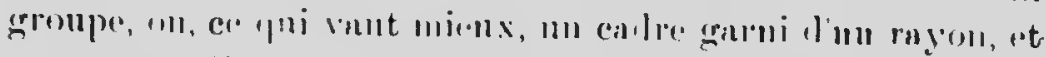
nII II)

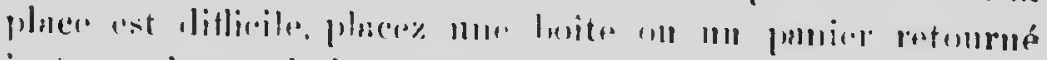

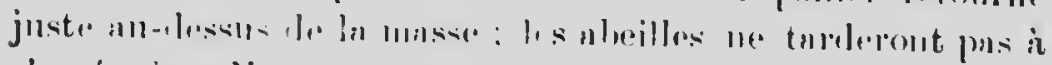

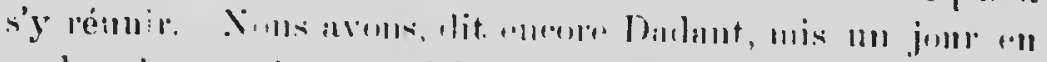

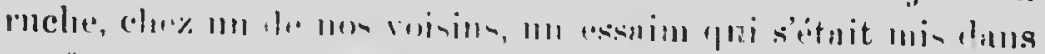

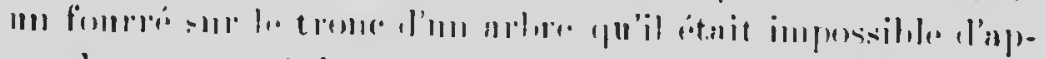

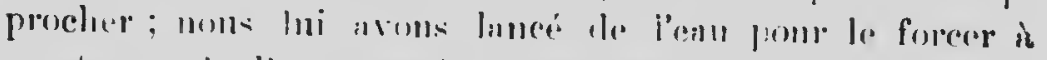

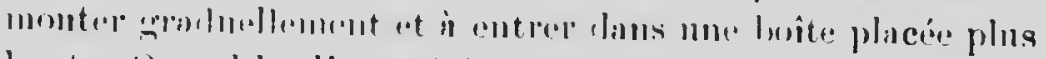

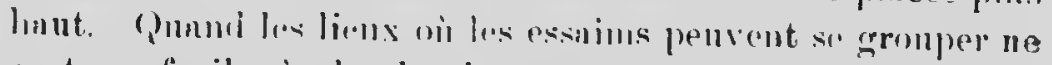

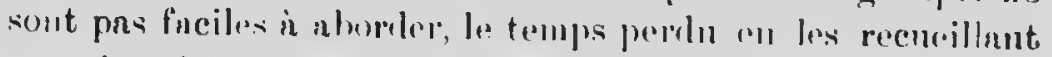

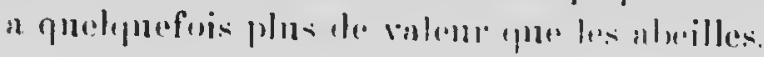

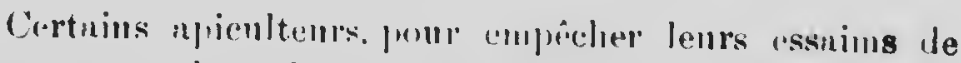

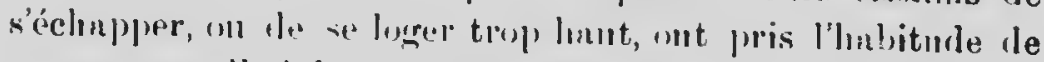
couper une aile in loms reines. М. Lanirstrotli n employic ce moyen pour commaitre l'âs̆ de ses ruines. Il compnit d'm coup de cisou une der quatre niles l'unc jelune reine. J'muné suivunte, en exsminant ses mehes, il coupnit la suconile nile lu môme côté; il compait les rleux autres la troisieme unuéc. Comme In vieille reine ne quitte la 
ruche que pour necompagner un essaim, In perte de ses niles ne ruit uucmement $\dot{a}$ ses aptitudes et ne diminue en nucume furon linttuchement de ses filles. Si, mulyré son inpossibilité le prendre le vol, elle vent émigrer, elic ne peut que tomber sur le sol un lien de s'élever dans les airs. Si les nheilles In tronvent, elles se rnsemblent antour l'elle, alors l'essuim est fincile à récolter pour l'upiculteur ; si elles ne la trouvent pas, elles retonrneut ì la ruche pour attendre la muturité d'une les jemes reines

Cettr mithomlo prut être suivic, à In condition que le sol

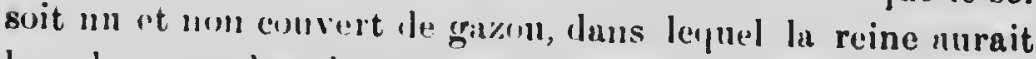

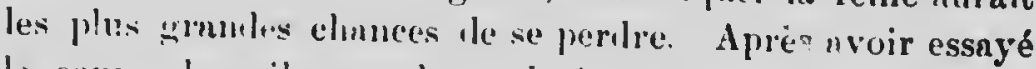
In coupe des ailes pendant plusieurs anmés, nous l'uvons almulommer comme lommat plas de trurail que de profit. Cepemlant nous commuisomis phesiens grands upicultenrs qui praticunont cotte methole. (Darlunt)

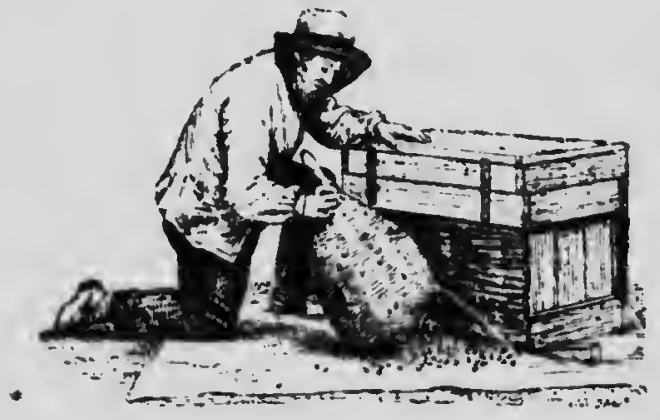

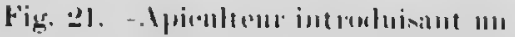

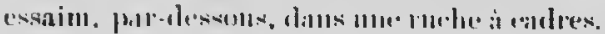

Mise en ruche d'un essaim. - lan rucle a itiv prenlublement nen-

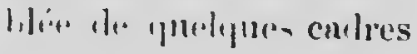
garnis al" cire gruffée Quatle contres de 17 par 1.2 punces snflisent pour un es-num ordinuire; il rialut mieux ne donner que juste ln place necessaire et n'njouter un nouvenu carlre qu" lorsque les premiers sont entièrement construits. On peut lonner des calres simplement nmorcés, inais la ponte et l'emmagasinement $d n$ miel iront plus' vite si l'on donne les feuilles et même, nu centre, un rayon tout bâti. Les partitions doivent flnnquer les cadres de chaque côté, (figg. 21). Si l’on secone les abé:!les sur lon drap devant l'entréc, on reconve la rucle avant de les 
secouer. Noms arous I linhitude de seconer l'essuim directement duns la rucle et écartons les partitions en luut pour faire entonnoir; nons les rnpprohoms en-luite petit à petit, en nous aidant un lesoin de l'enfunoir pour diriger les abeilles. (fig. 22). Pnis In Inclir est reconverte et le soir nons

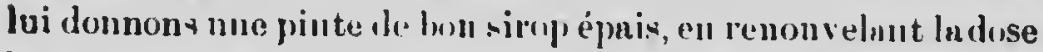
le lemlennin soir si lea uheilles nont ñ récolter dehors. Si l'on a recours à la métlus le Heidon, dëcrite plus lanut, c'est du niel et non dn sirn yn'il fint domer, citr la nonreiture

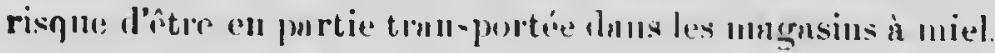

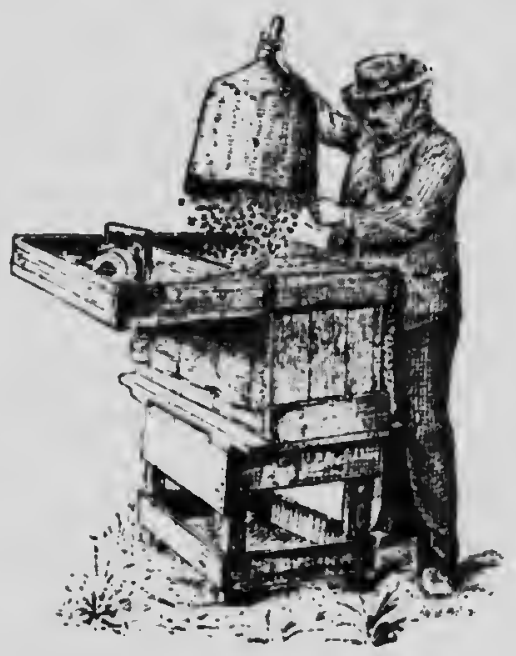

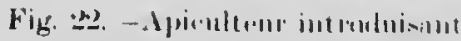
mo ex:

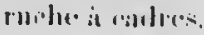

Lon ensuim mogron piese t:bis. "19,000 ubeille. envi.

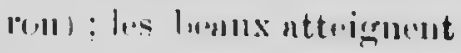
lict sthe. sidlinx essuims,

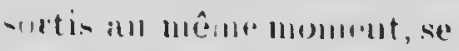

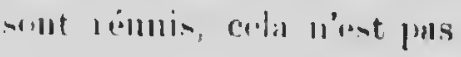

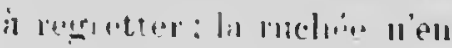
indrar yur miens ell ex yninlle hitim plus vite at

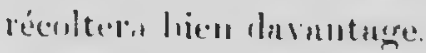

Les essalums secomlaires et suivnnts sont snjets à repartir le lemenumin ou mone ilus tam à la suite 1. 1+111.jenne a ciale enl quête l'mon com. On les retient

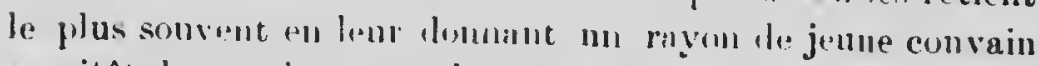

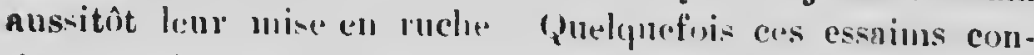

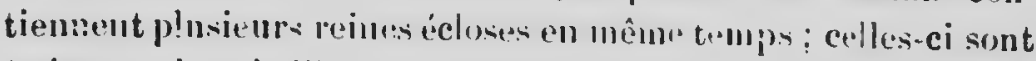
tuées pur les uhtilles il l'exception d'ume. Unr reine vierge est géuéralemrnt neceptée sans préliminairess, même par une vérituble colonie (orpheline), nais ì ln conclition d'être présentée daus les fremieres heures qui sulvent sa naissance.

Les essuims secomlnires sont somvent assir\% forts pour 
fuire de lonmes ruchées lans la saison, mais il 11 en est pus le même des essuims suivnnts, qui sont grénépralenent fuibles et qu'il vant tomjons mieux prévenie on rendre in la sonche, in moinz qu'on me venille an protites pomp los jennes reines gu'ils posierlent.

II ne fant poss perdre de vne que celni qui vent finiro le lommes ricolles doit éviter nutnot que gussible l'essnimage.

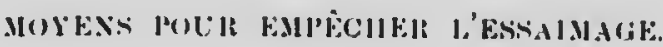

Ponr empêcher l'essuimagre, il est indispensalile de prévenir tont malnise, tout besoin, nvunt quelucolonie le ressente; car noms nvous remurqué, que quand les nleilles ont grngné ce fu'on a nommé nvec mipon la fijve d'exsroimulgr, il cst

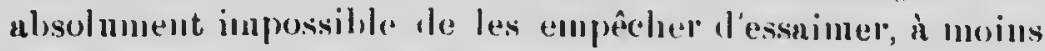
que le temps ne devienme lifinorable in la proluetion dn nectur lians les flinis.

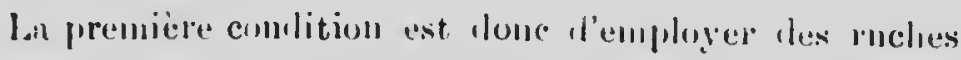

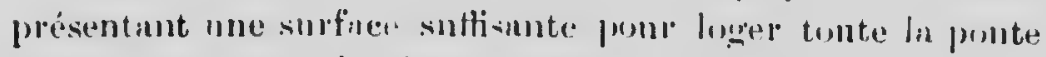

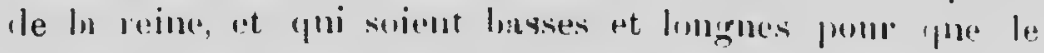

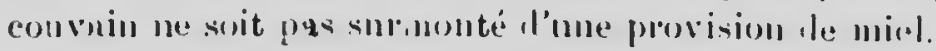

Ian seconde comblition sime qui mon, c'est de lommer ans ubeilles des logons villos qui soient nnsi rnpproches dn

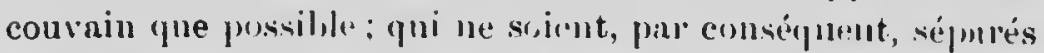
le lni ni par , lи minl ni jar mue plunclue : et l'entretenile ces

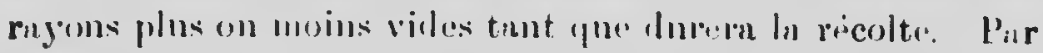

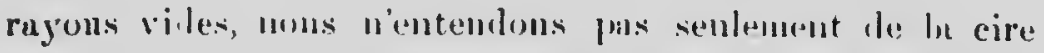

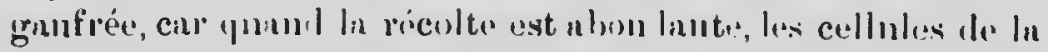
cire grufifée no sillongent pas nsse\% vite; noms vonlons dire de vois rayoms, nux.jucls on pouron mólunger quelpues carlies de cire ganflée.

In troisiene combition, cest de ne pas nttendre que les ubeilles nient lesoin la ces myons, mais de les mettre unssitôt que lu principale réculte commrnce. ()/ recomnait que ce moment est nrivé, qunud on voit le dessur des rayous 
de la chambre í couvain hanchir par la cire nenve que les abeilles $y$ njoutent pour les allonger et $y$ mettre dn miel. Il vaut mienx placer les myons de surplus trop tôt que trop taril.

Les conditions nccessoires, c'est d'empêcher une trop grmale production de minles; entin, c'est de tenir ln ruche biell ventilée, duns un endroit oì elle soit ì l'abri des rayons din soleil de milli, on de lombrager de quelyue fuçon, comme en plantunt me treille on des a.brissenux ilont le feuillage la garuntisse des myons trop ardents, on en la courrant d'un toit provisoire.

Nous empluyous les moyens précélents, lit Dadant, depuis de longues unnées et réussissons ì n’avoir pas plus de deux ì trois pour cent l'essains. M. Bertran l, directeur de la Revue Internationale d'A pieullure, à Nyon, Suisse, et M. Cowan, directeur du Brilish Bee Journal, de Londres, ont tous denx alopti cette methorle uvec les meilleurs résultnts.

Eu supprimnnt l'essnimnge matmrel, on se dispense d'une surveillance très assujettissunte et on évite l'affiłblissement des populations an monnent le la granile iniellée, ce qui est, comme nons l'uvons léjir explịué, l'une importance capitale au point le viue de la récolte, principalement dans nos contrées oi ln miellée est le courte durée. II funt alors, si l'on ieut ungmenter le nombre de ses colonies et n'entretenir que les reines jeunes et fécon/les, recourir à d'uutres moyens de multipliculion et d'elevage.

L'essaimage artificiel est lusé sur ce principe qu'une colonie l'ubeilles privée de sa reine en élève de nonvelles pour la remplace", si elle est en possession d'uufs on de jeunes larves d'ouvrières. Cet élevage ne peut aboutir qu'aux époqnes oil il existe des mâles pour féconder les reines, et il ne se fera daus de bonnes conditions que s'il $y$ ' récolte "u dehors, ou si les ubeilles sont nourries artificiellement. 
l'remière munire-l'oici comment peut s'y prendre lo commenrant pumr fuire un essaim: A l'éporgue de ln grnnde Hornison et pur une helle jomrnee, uprès nvoir fait choix d'une forte colonie richu" an couvinin, ce pui est mue colstition escentielle, il in cherche la reine et place le rayon qui la porte", avec les uleilles inj le reculvent, duns une ruche vide. Il proml un seconl riyon de cournin, muis suns les rbrilles, et mêm. un troisième si in rnclie en possède plus do cinq contenunt du cournin 1, whs un rayon de miel: il les met ì côté du promier et ferme ln ruchre, sans onblier d'en. clnver les rnyous entre denx purtitions, puis il installe cette ruche à ln plnce de celle qui vieut d'être divisée.

1)ans cette lemière, qu'on lésigne sous le nom de souche, les rnyous restunts unrout été rupprochés; ceux it cournin seront grompés un centre et l'uu l'eux mu moins levrn contenir des reufs. Elle sera instullée à un nutre endroit da rucher. Ses butineuses retoumerout in leur ancien emplince. ment et renforceront l'essnim, tamdis que ses jennes abeillea, se sputunt orphelines, élèverunt de nouvelles reines. Lat colonie montrern fort peu d'activité penilnnt quelques jours, aynut perdu ses butineuses; il fiudra lui donner uu peu d'enu dnus le nourrisseur, et même du miel le soir si le temps est manvais penlant l'élevage des lnrves royales. II est intiniment pen prolunble qu'elle jrtte un ess:lim, malgré son élevage le reines, nynut en sa populntion consilérablentent réduite. Elle se refern petit ir petit par l'éclosion dn convan gui lni restrit lors de sa division, et dn reste on fourm ta renfurcer plus turl en lui lommut un rayon de couvain pris duns une nutre colonie.

An lieu de lnisser In molle orph line élever des reines,

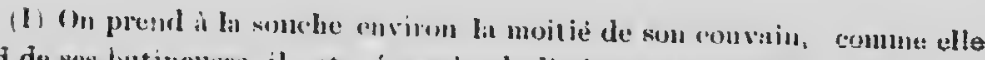
perd de spes butincuses, il est nécessaire de diminner la proportion du convain par rapjort an nombre les nonrrices busskes four le snigner, vi qu'elles neront s(rulkes jomr le réchanffer. 


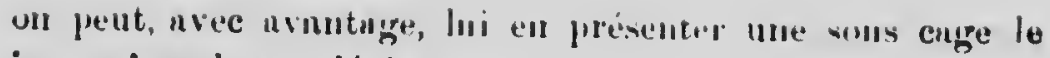
jour mène le sun léplncenent.

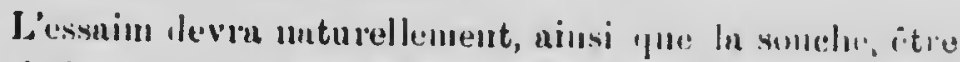
surveillé un point de rue les provisinns et le l'ngrandissisment de l'hubitntion selun les liesoina.

Deuxieme manière- Une nutre méthode ent celle que recummunde ell premicer lieu le live de $\mathbf{3}$. Dailnut, l'a beille et la Ruche de Langstroth.

Quelques jours avant l'époque habituelle de ln sortie des essaims nuturels, c'est-ǹ-dire lorsplue les ruches sont bien peup!ées, on prélève toutes les abeilles l'une forte colonio que nous lésignons par $A$, et on les met lans une nouvelle ruche ì la place de la suuche. Celle-ci est mise elle-même a la pluce d'une nutre bonne colunie B. qui est portée daus un nouvel emplacement.

l'our fuire le prélèvenent des nbeilles, on preml d'ubord le myon portant la reine et on le plate tel quel dans la nonvelle ruche, arec quelques culdes nuorcés on garnis le cire ganfrée, ou mème nvec de's rayous bitis si un pll possèle. Les ubeilles des antres rmyms sont hrossines an un drup devint ln inclie, ou secesuées lorsque les rnjous ne contiennent pas trop le rectur fruiehement récolté.

Les rayons déluriressés des abeilles quilis portaient sont inmedintement rendas in la souche, mais il we sern que ben d'en domer in l'essinim un contennut alu miel.

Dens colnoies participent ninsi is la formation l'un

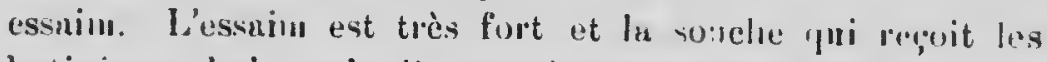
butineuses de lin ruche li, reste éralement tries penplée.

Elly pourn bien essaimer si onl lni licisse tont son couvnin, mnis on dimimern ce risque d'essubmage en raison

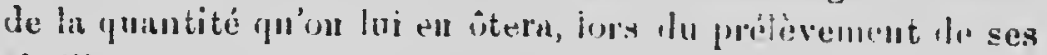
abeillas, pour l'ajouter in l'estrim formé.

Ln ruche b déplacée perd sé. lutincuses, mais sn popu- 
lntion se recunstitue très promptement par l'éclonion jomrna. lière de soll couvriu.

Troisième munière-Voici entin ln méthole simplitiéc à laquelle M. de Layens donne la préférence :

Dıns le prucédé que nous allons décrire, on se proposo de fuire un essain artificiel non pus nu moyen d'une seulo ruchn, mnis ell y' fuisnut concourip deux forley rushey.

Pur ce système on obtient en détinitive trois ruches dont l'ensemble, contiendra souvent untant de miel et de cire a la fin de la stison quen aurnient contenu les deux ruches einployées '.

Supposons que nous ayous choisi deux fortes colonic. du rucher, qu'une visito prẹ́alable nous a montrées riches en couvain et en population.

Ce seront par exeinple les ruches $A$ et B, représentées dans le tubleau ci-dessous. Nous aurons placé non loin de lih, une

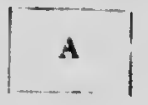

A, forte rus:le

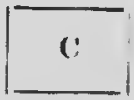

C, Kurhe soucler nyant regill les radren de Ii et reco. vant les alsulles de A yui étaient in la récoulte; clle formera une mire.

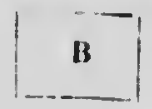

$B$, forle rucles.

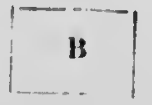

B, Kuche devenue exactim artificipl.

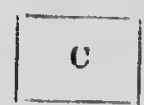

C, Ruche vide diuboillos et sans cadies.

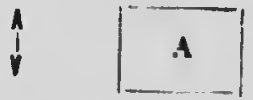

A. Rnche deplacte ayant perdu les alvilles qui étajeut da lairécolte, ot qu'on transportera au loiı.

ruche vile d'nbeilles et sans cadres C. Après aroir enfumb́ la ruche h, lous "ll retirons successivement chacue cadro avec les ubeilles qu'il porte et nous brossons les nbeilles duns

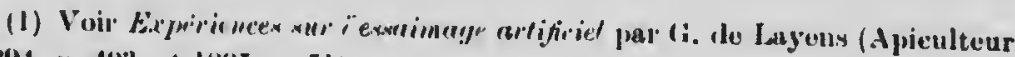

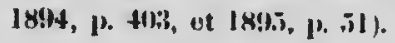

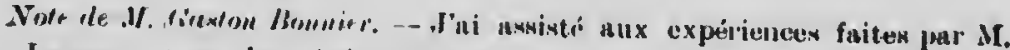
de Layens, et en les répétant duns mon ruclec, j'ui olsteun len mômos résultatw. 
cette rucle B; mons mettons suceessivenent les chlres ninsi

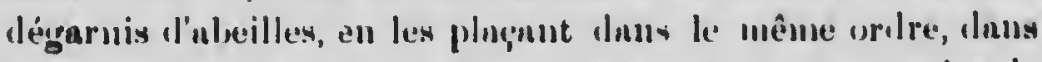

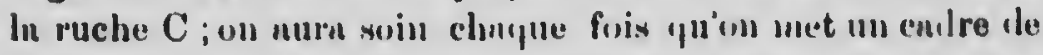

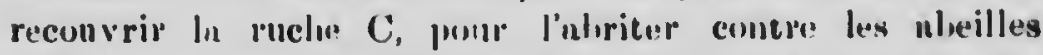

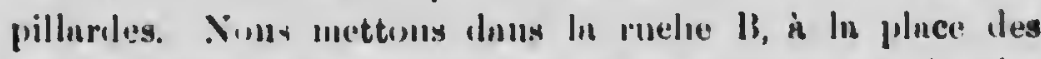

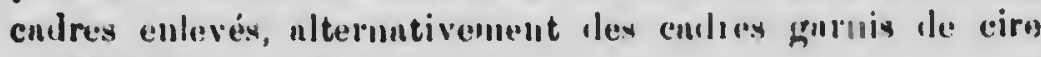
gnufrée et des conlres musucés.

Il est bon de laisser dan. In rucle II in rayou contensant a ln fois in couvinin de tout hige et lu miel. On le met a l'avant-dernière pluce, ilu côté de la porte ouverte. '

La ruclse $\mathrm{C}$ contiendra lone alors tous les carlres yu'avnit primitivement la ruche $B$, sulf $\| 11$ q ju'vu $y$ a laissé.

La ruche $C$ est ulors mise in la pluce de l'nutre forte ruche $A$, que nous allons imettre asse\% loin sur 11 nonvean plateau porté par un tubouret (tublenu page 72 ).

Que vin-t-il se pusser!

Les abeilles de la ruclıe $A$, yui étnient í ln réculte, reevieunent ì leur plnce nccontuméc, reconmnissant leur platean, entrent dans la nouvelle ruche $\mathbf{C}$, et $\mathbf{y}$ trouvint du couvain en graude quantité, se mettent ì le soigner. Elles manifesteront tout d'abord une certaine ngitution, purce rju'elles ne trouvent plus de bière duns ln colonice, mais nu lout le peu de temps, elles se décileront ì construire des cellules maternelles, nu moyen du jeune couvinin qu'elles unt à leur disposition.

Pnssons ì la ruche A : c'est la ruche forte yni n été déplacée et installée plus luin Inus le rucher : cette ruche a conservi tous ses royons arec les ulue illes qui s'y trournient et elle a perdu les nbeilles nllant nux clmmp qui sont entrées dans ln rucle C. Mnis nu hout d'un certnin temp's, grâce a l'éclosion dn convinin, on verrn cette ruclse repremlre peu a peu une gimule netivite. Cette ruclse $\mathrm{A}$ iéplacée essinimera très rurement.

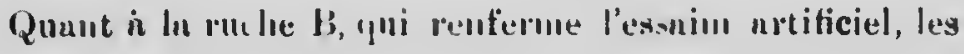

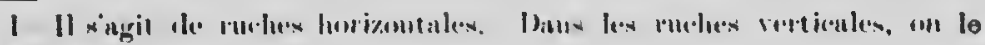
met all cenlire. 


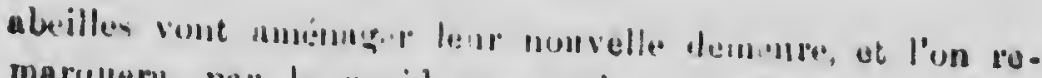
marfleru, par le rupile viset-vient de l'entrée, qu'elles

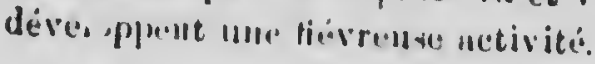

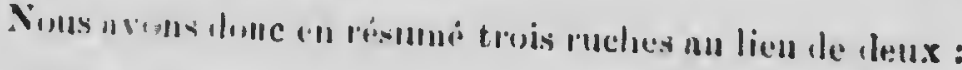

I" Une ruche l', riche en consuin et qui va faire une

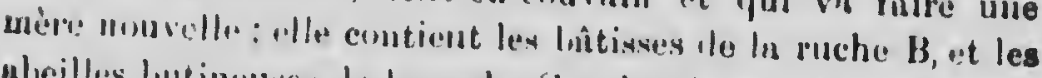

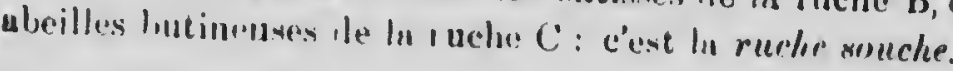

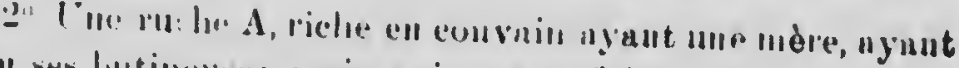

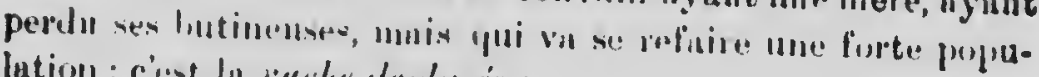

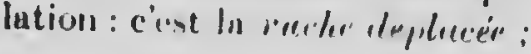

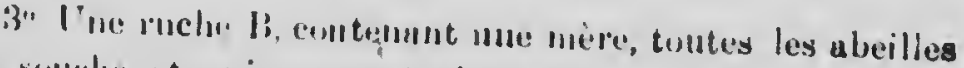
de lu sonche, let illi in construire de nouvelles hattisses duns

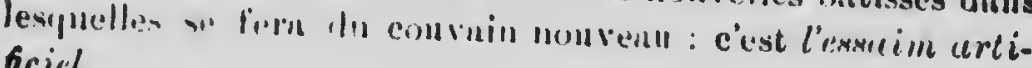
ficin.

ruch
mob
Ainé
tage
leur
la p
abeil
conil
bas
telle
ruch
dont
a fui
pour
déjà




\title{
CHAPITHE CINQUIEME
}

\author{
I) RS RLCHES
}

\section{Huches volgaires.}

Nous allons muintenunt étulier lifferentes aptosd ruches, depuis la ruche vulgnire $\mathrm{d}$ lu ruche verticalt it ayous mobiles, cette lernière étunt seule utilisée de nus jontar en Anérique et recommulable, en rnison des nombrenx «mแtages qu'elle poswedo sur les nutres.

Los nbeilles à l'étut unturel étublissent le plus sunrent leurs colonies daus les vienx trones creux des urbres; mussi, la première illée de cenx qui ont cherehé à cultiver les abeilles n-t-elle été smo fonte d'instuller ın essuim lnus les conditions maturelles.

Un tronc diarbre crellse in l'intérienr, scié en lonut et en bas et reconvert d'une plaque dre bois on llun gross pierme, telle fut la prenière inclue. On trouse ancore de telles ruches dans certrines contrées.

Là un croît le Chêne-liège, en Frunce, ln muièr. fincile dont se détache l'écorce ép:aisse et imperméable de cet arbre a fuit préféros mx cultivat urs l'emplosi le l'icuree de liège pour former l'enveloppe le: I'hulitation des ubeilles. C'est déjà une ruche plus trarnillée que le simple trone l'urbre.

Dans les antres riggion; et purticulièrenent dans les pays montngnenx, on n construit des ruches plus huntes yre larges, formées vimplement l'u. nissembluge le quitre 
planches avec un couvercle eloné pur dessus; c'est eucore une ruche primitive unuis l'une forme plus régulière.

En leaneoup d'nutres contrées, les eultivateurs ont donné comme logement aux abeilles mue enveloppe de forme arromlie, souveut pointue vers le linut, purfe is de forme basse, et qui est fuhriquée soit avec de lu prille soit avec des brnuches Hexilıles régulièrement entrelncées; Cest cette forme de melıe qui u été la plus répnndue nvant l'iutroduc. tion des unches in culres mobiles.

Toutes ces ruches, depuis le simple trone d'arbre primitif jusqu'à la ruche en osier la plus soignée, sont lésignées sous le nom de ruches vulyuirus.

Atin de consolider le's lnitisses, on a lhabitude de mettre dans ces ruehes des buguettes de bois disposées en travers a l'intérieur.

Pour que les abeilles soient plus ì l'ubri des arimux qui pourraieut venir les attaquer, on place généralement les ruches sur uı support en plunches appelé plat ...;, muintouu au-dessus du sol d'une manière on d'une nutre.

Comme les ubeilles mettent lenr provision de miel audessus et sur les cûtés du groupe qu'elles forment, on comprendra fucilement les prineipaux avantages et inconvénients des diverses ruehes vulguires dont on vient de purler. Les ruches c'n tronc d'urbre, ell liège on en planches ont un dessus qui pent s'enlever comme un eouvercle, ce qui permet de récolter le miel qui se trouve à la partie supérieure suns trop dléranger les uheillcs. Les ruches, frites en cloche, en puille (fig. 2:3) on en osier (fig. 24), ont une forme qui permet au gronpe dubeillss de eonsommer peu ì peu sn provision de iniel pendant l'hiver. En effet, la tin de l'automne, le gronpe d'ubeilles se trouve surtont itu-tlessous du miel, et s'élève en le consommant pell ì peu. Ise groupe d'ubeilles montant inus la ruche in mesure ighe s'avanee ln mauvaise saison, se trouve, grîec dे la forme de la rucher, à proximité 
du miel qui Ini est nésessuire. Mais à chité de cet avantuge, il fuut signnler linconvénient y!ne ces ruches présentent pour la récolte. Si l'on ne vent pas tuer les uli:illes, et si l'un no sait pas employor penr leur enltnre les bonnes méthodes

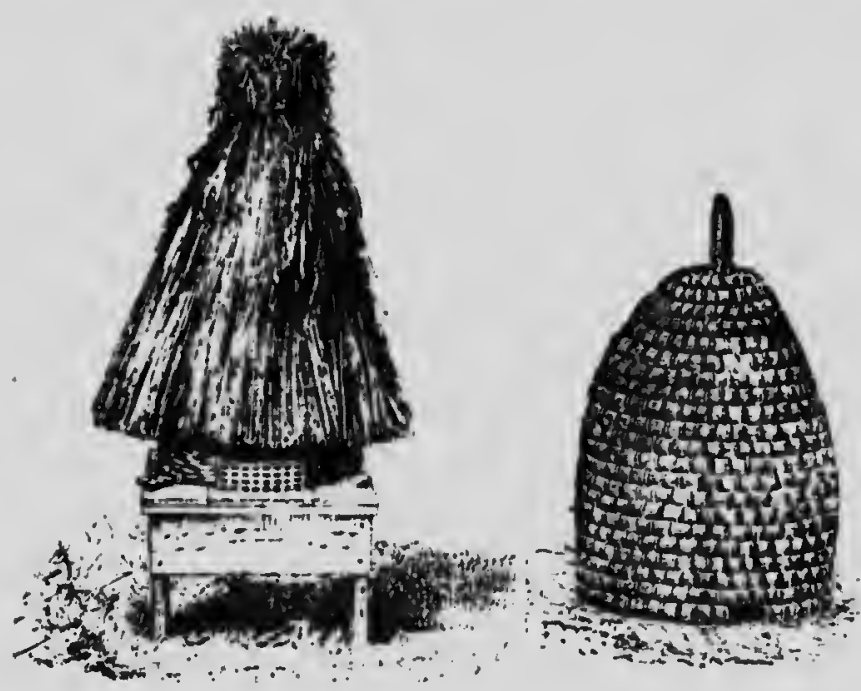

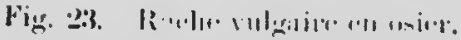

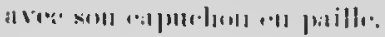

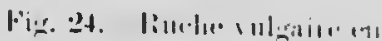

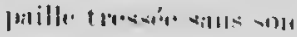

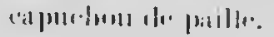

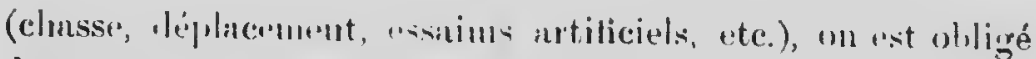

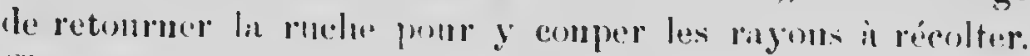

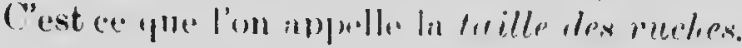

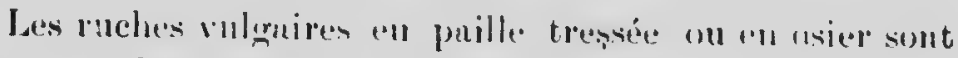

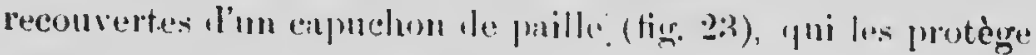
contre la pluie et contre le froil.

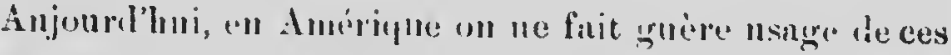
ruches que l'on a remplacées avec avantage pur des ruches a calres molviles, notsi allons donner ln description de deux ruches de ce typu lont le premiar sert surtout en Europe (Belgique-France). 


\section{$-78-$ \\ RCCHES A CADRES MOHIIFS.}

Il existe un grand nombre de systèmes de ruches a cadres; la ruche que nous allons décrire est l'une de celles qui se prêtent le micux d̀ une culture à la fois simplifiée et productive, on l'appelle quelquefois rucke française.

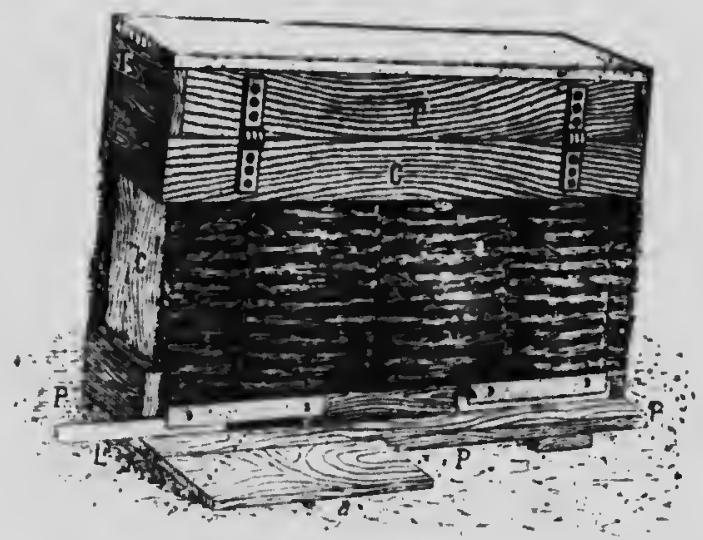

Ruche de Tarous.

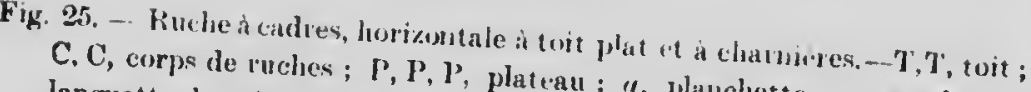
C. C, corps de luches; P, P, l', platerau; ", planchette ; e, entre : I, languette de métal pernettant d'ourrir plus ou moins l'entréc.

Cette ruche (fig. 25) se coulose d'ure enisse en lois eans fond dont le couvercle formant le toit de la ruche (T,T, fig. 25) est relié à la caisse par deur channières que l'on roit sur la figure. Les deux fuces les. 'us grandes de la caisse constituent ce qu'on aprelle : le detant ct le derriere de la ruche; les deux finces les plus petites sont appelées les cotés de la ruche, et la caisse tcut entière forme le corps de 1. ruche (C, C, fig. 25). La figure 27 raprésente ce corps 
de la ruche isolé. Une gminde partie da devant et du derriere de la ruche eet reconverte de paille conme on le voit

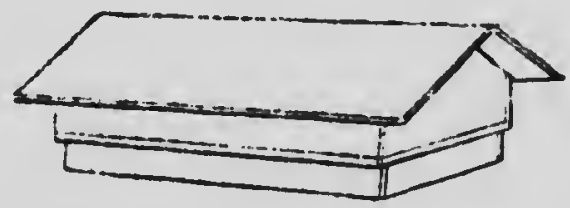

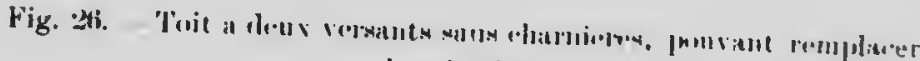
lo lovit plat.

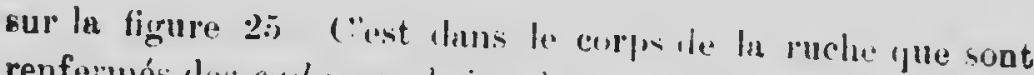

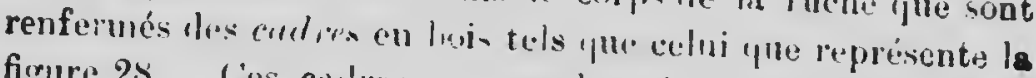

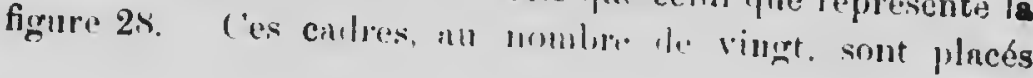

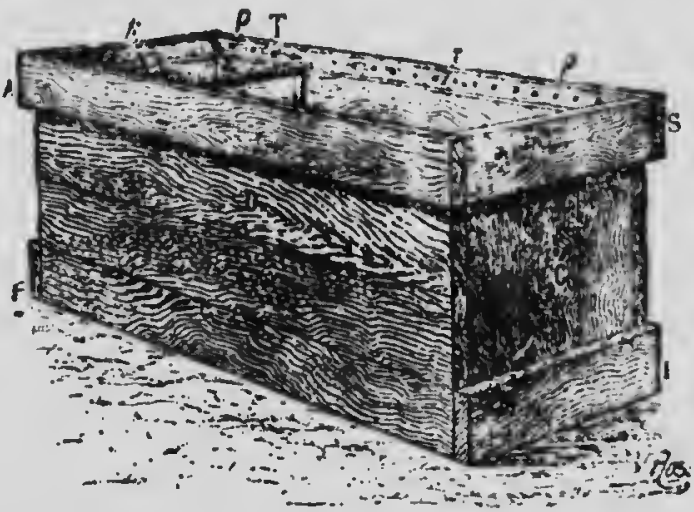

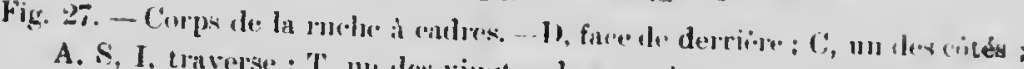

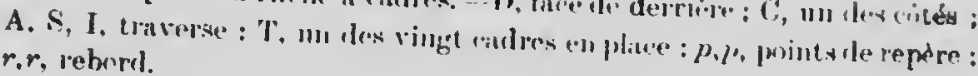

parallèlement aux crités de la ruche. () ll vit l'un de ces cadres en place dans le corps d. la ruche, en T sur la figure

Enfin, cette caisse sans fond regrese siniplement sur une 


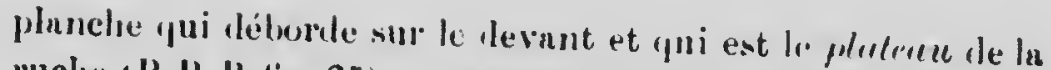
ruche 'P, I', l', tig. 25) ; mne petite plunchette a, sur laquelle nrrivent les nbeilles, est tixée in unuclur et ell avint du platenn.

(1) pent remplneer le toit plut it eharuieres par n!n toit is denx ressants snit ha larnièreg suit sans chanuineses (tigr. 26i).

Noms allons maintenant lécrire (hocume les parties poincipalos de la incher.

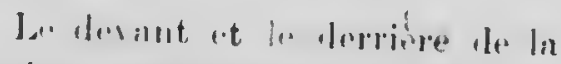

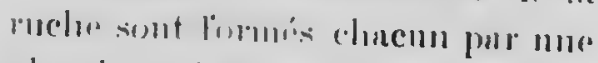

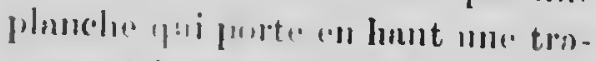

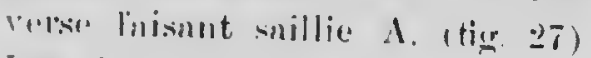

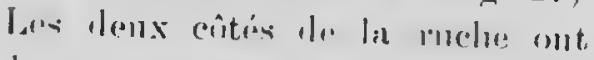

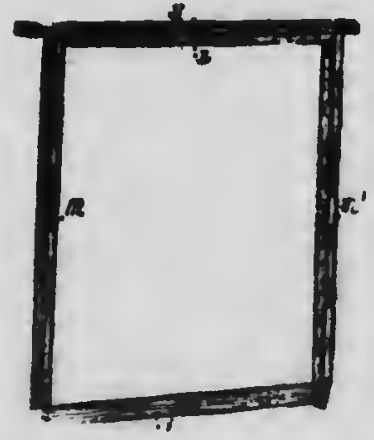

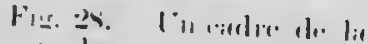

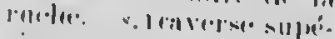
riemle: A lawerse de

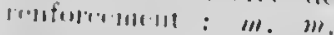
montalils: $;$, tonvelare intivipinill.

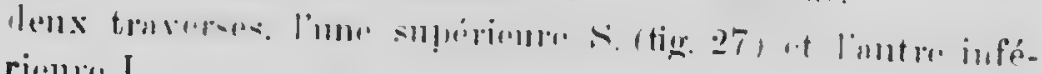
rivill. I.

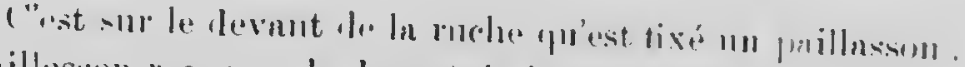

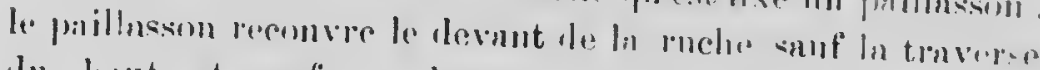

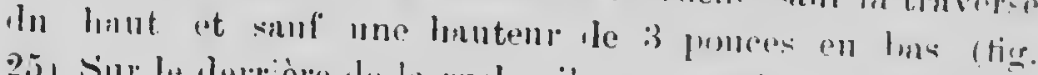

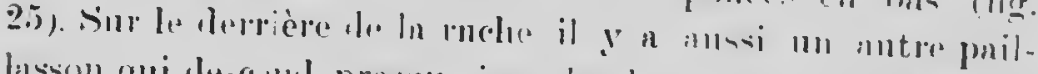

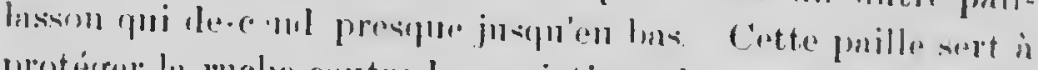

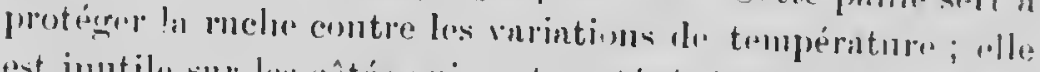

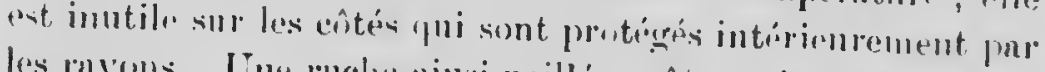

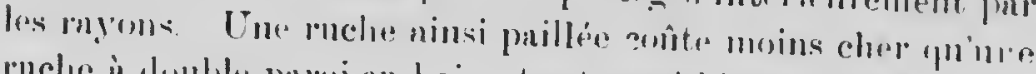

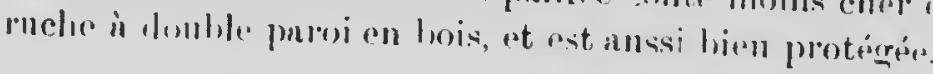

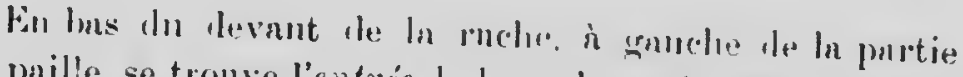

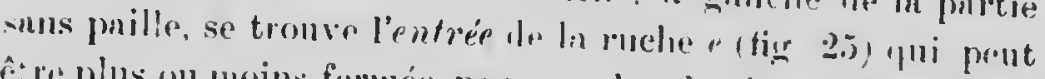

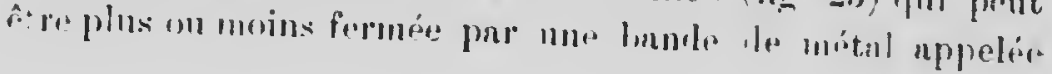




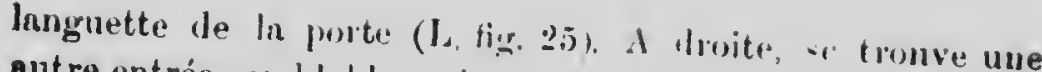
autre entrée scmblahle. qui pent remplacer la premiere!

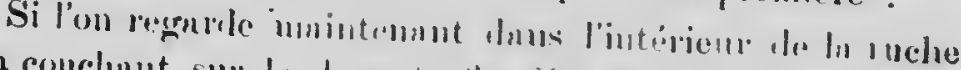

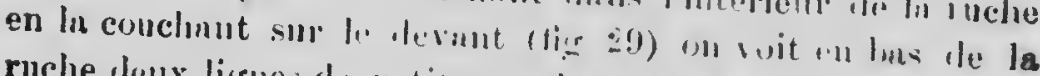

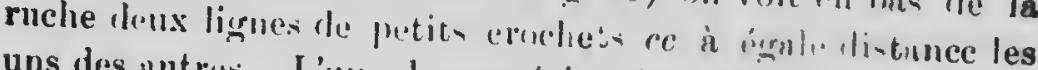

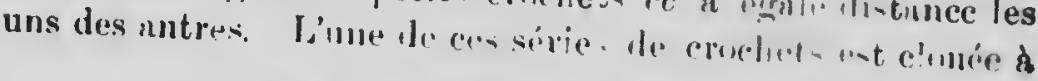

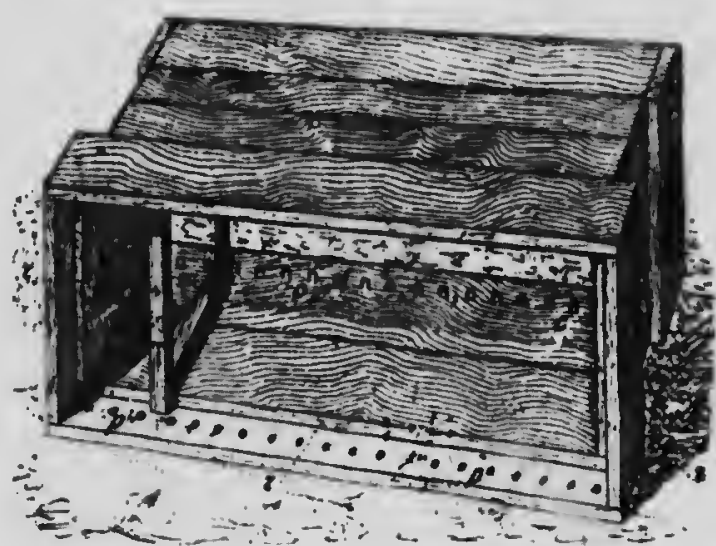

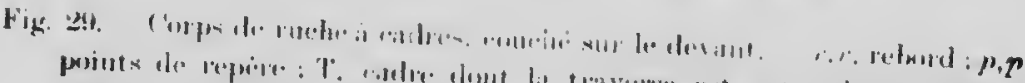

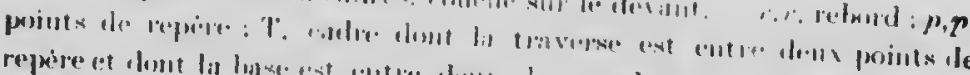

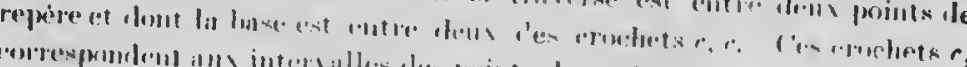

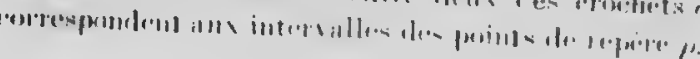

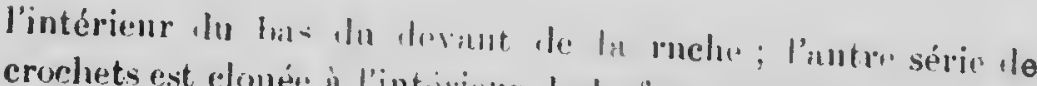

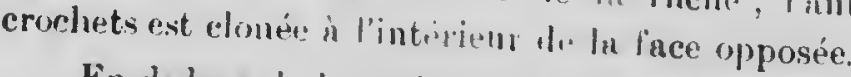

En dolnus, le linut du comp de in ruche porte un rebord intérieur tont nutour ( $r, r^{\prime}$, tig. 27 at 29); nu-lessus de ce reborl et a lintérienr du corps lo la ruche se tromvent deux

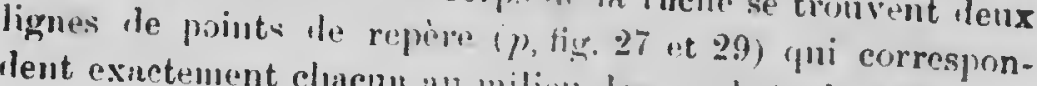
dent exactement chacur an milinu les conchets du has.

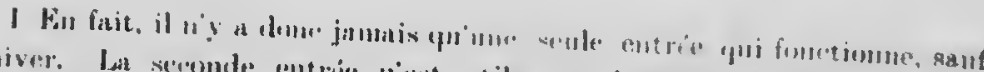

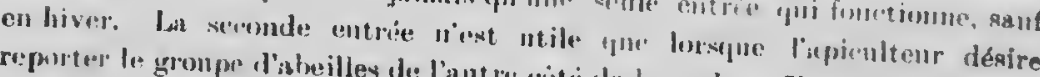

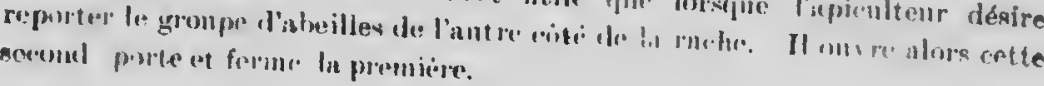




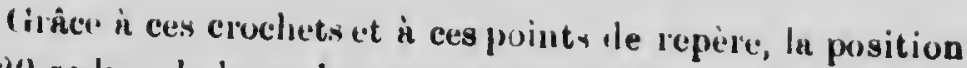
des :0 corles de la ruche se tronve nettement indiquée. On pluce chaque culle de fugon que sa base vienne se plucer de cluque côté entre deux cruclit ts tandis que la traverse supérieure du culre prend position de claque côté entre deux points de rejière corresponelants. On roit sur la tigure 27 un endre uinsi placé duns su position niturelle.

Quandles cul res sunt posés, il reste cuntre leurs traverses supéricures un intervalle qu'on ferme par des lattes de bois placées sur champ (royez tig. 30). Sur le tout, on met de vieilles comvertures de laine on un prillusson.

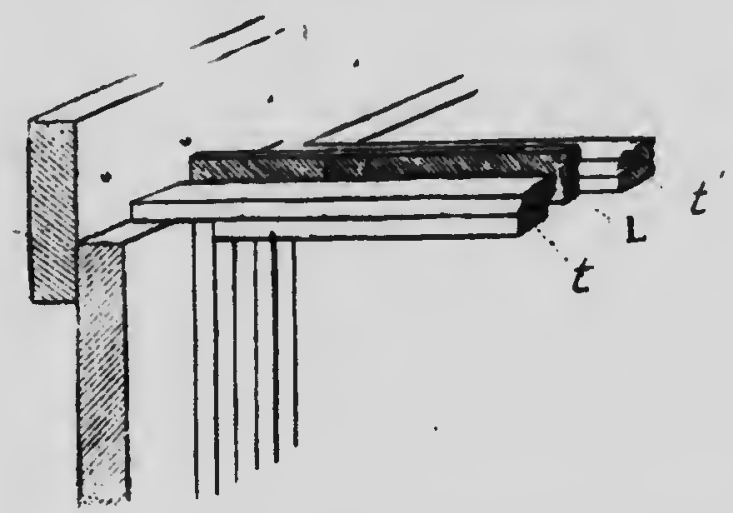

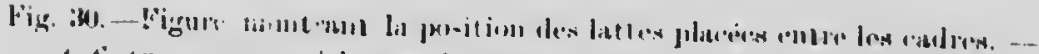

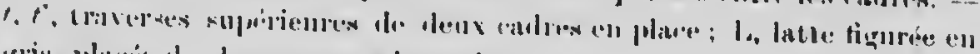

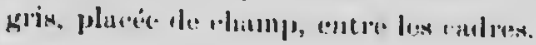

Le toit de la rilelic ent forme de platies limmes de bois nssemblées, et reonvertes d'une feuille de toile mince galva. nisée, tiguréc par une teinte grine sur ln figure 26. In hinuteur de ce toit permet le placer fncilement des nonrris. scurs et les sections ponr le miel en ruyon.

\section{RUCWES MADAST OC VERTICALES}

Liilée première de la ruche à calres inobiles est due a François Huber de Genève, le père de l'apiculture inoderne, 
muis sn ruche ir feuillets ne fut unere utilisée que comme instrument d'ohservintion et ee n'est pue cimplunite ans plus tard qune la ruche is colles, tolle que nous l'empluyons, tit son intrée duns le domnine de l'upiculture protique.

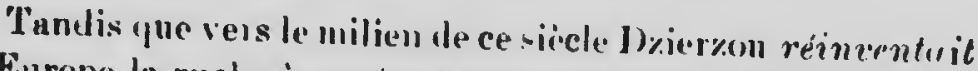
“n Europe la ruche ì porte-rayons mobiles, déjn proposée a In tin In siècle dernier par 1)ella Rocen muis oubliée, Langstroth, aux Etats-Unis, inventnit, mom snus en uttribuer la presiniere irlée in Huleer, In ruche in conlres que la majorité des Américnins emploient cncore nujomblumi in pen poè telle quelle.

Un «ntre npiculteur du même prys. Qninly, qui s'ocenlonit d'ubeilles depuis l'unnée 1830 at "roit mopté in ruche Lungstrotl , les son nppurition, publinit, il $y$ a +7 uns, la première élition de son ouvrage Los. Ilystereg de l'A piculture $\therefore$ pliquers, dans lequel urous trouvons ln description d'une ruche lanugstroth moditiée par lui. (ette rucl e diffërnit de eclle le l'inventeur en ce que sn ennstruction étnit simp'itiée et yne lis colles, réluits an chittre le luit pour ln ehumbre i convuin, étnient un pen phus grands dans les denx dimen. sions.

Cette ruche Quinby fint nloptée por un grmol npienltenr

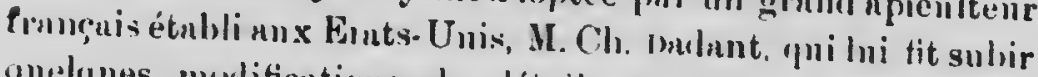
quelqunes morlifications de létnil et portn le nombre des

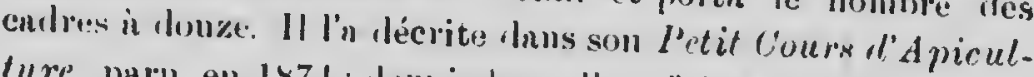
ture, paru ell $187+$; depuis bors elle "linit sou chenin dans lo monde des npienltems et c'est sons le nom de ruche Dulant que nons ulloms is motre temr la présenter à nos lecterrrs.

Quelle qune soit l'exactitule des lemnets que noms indi. 'prons, nous ne pourons que cunseiller à cenx qui venlent s'udonner \& l'apienlture, l'nehrter an moins une on deux ruches fuites par des personner qui commissent c. metier,

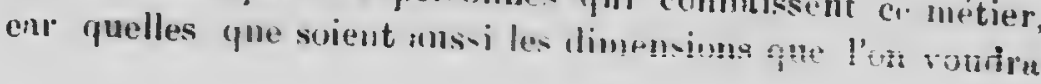


accepter, il sern phins fucile nlors de se remlic: compte des détnil ine requierent le. Incliex lijen construites. (Kig. 31).

Corps de ruche $t$ t plateau - I te corps de ruche est

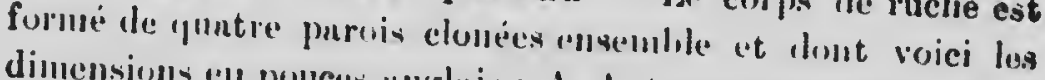
dinensions ell pouces mughis: $A, A$, traverse silpportant lo

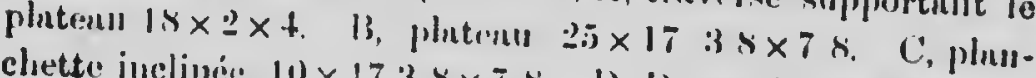

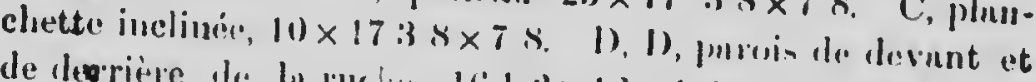

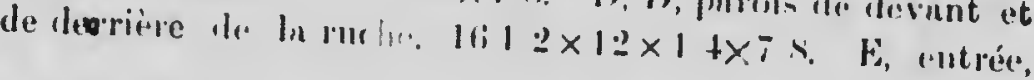

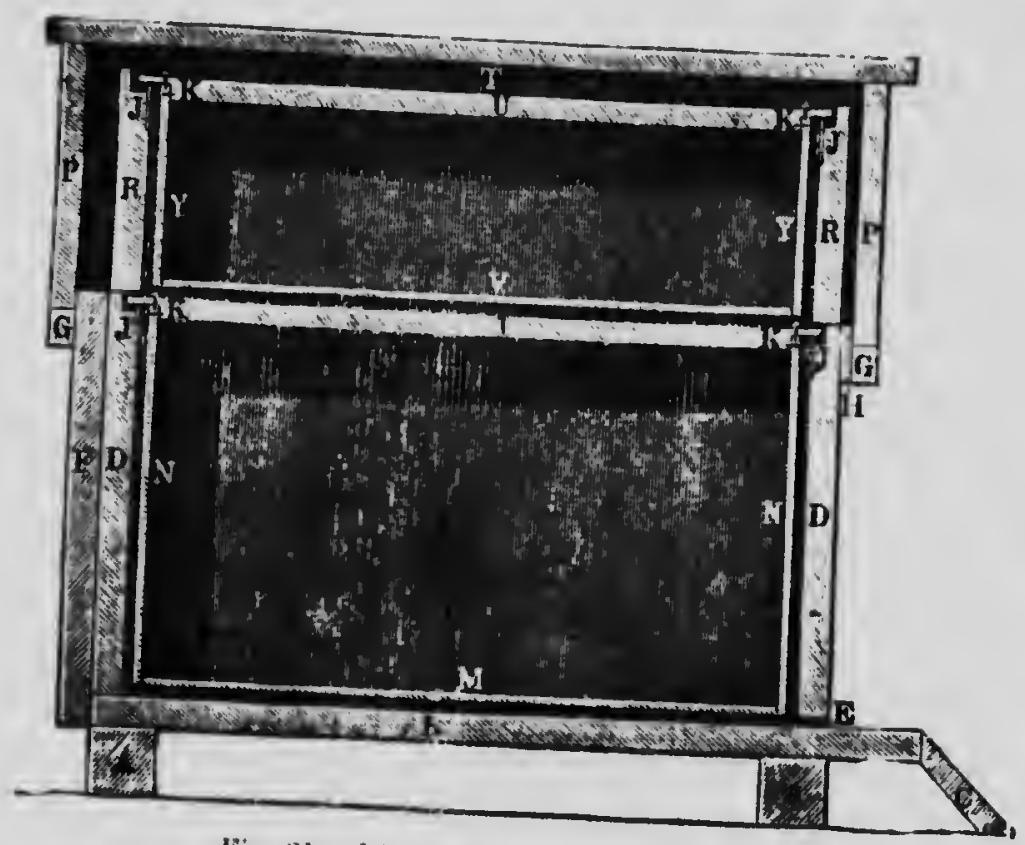

Fir. 31. Diazramm! , le la ruthe Hanlant.

Sxi, li. F, seconde paroi de derriere, $17: 3 \times \times 1: 318 \times 7, s$. G, G, latte's supportant le clinpitenn. II, lintw, $1,2 \times 13 / 4$ pour élargir le horil supérienr les ln mivi le terant. I, tra. verse supérienre in culre. $2011+\times 3+x i s . J, J . J$, fenillures, de $1 / 2$ do langur sur is s le luntour, putrillees dans les purois de devint et de derriore. at mmies de hues de ferde 3 t de luge, dipassant en liant de $1+$ et suppor- 
tant les uxtrónités des truveros supérienres lous culres. Si les fenillures ne sont pus mumies de lnues de fier, elles ont $1 / 2 \times 3 / 8 . \quad K K, K, K$, muntrent comment les montants du cadre sont ussembles nvec ln traverse supipienje. I, traverse

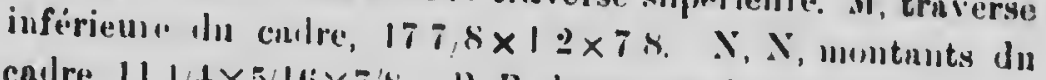

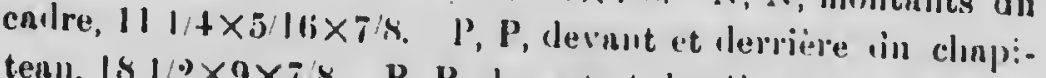

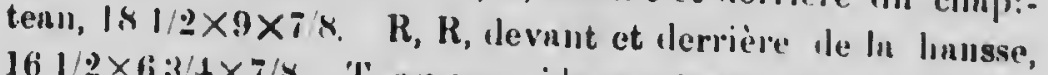
$161 / 2 \times 6: 3 /+\times 7 / x$. T. expuce vill nu-lessus le la liansse, $1 \mathrm{l} / \mathrm{s} U$, triverse inférieure du cudre de linusser, commue la traverse J. V, truverse inférieure lu culre do lıusse, commo In traverse V, Y, Y, montmuts du culle de halssse, $7 \times 1+\times 7 / 8$.

Liospace entre II et 13 est envirou de $1 / 2$ : entre D $N$, N J), V I, R Y, Y R, il duit être de :3, 8 On pent coustruire les ruches de différenter grmudeurs l'nprèi ce diagramune, \& la condition de méunger les espucex le ln largeur indi(fuée.

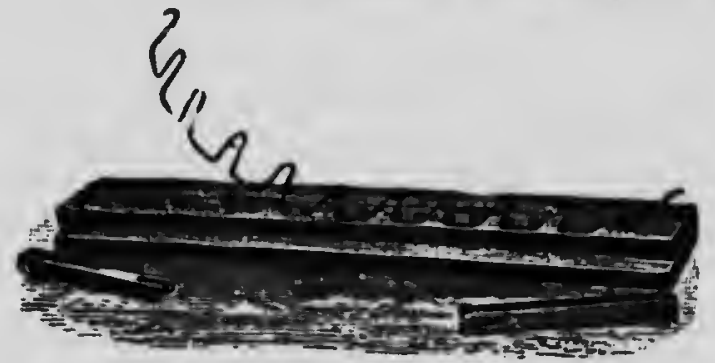

Fig. 3:. -.. Manicre de dégager le dentier.

Dentiers-équerres et agrafes. - Les cadres sont es. pacés entre eux de 3,4 ou 5 lignes. Pour éviter qu'ils se déplncent lorsquion renume la ruelse MM. Quinhy et Dnilant out clincum inngine un denties en til le fer qui g'udapte mu bas de la ruche et lans lequel les emlres s'engrgent. Pour fincommer le lenticr, II. Durlant se sert le lattes dans lesifuelles sont plantées nux ilistances voulues des vig autour despuelles ont fuit pusser lu fort fil le fer Les deux lattes portmut les vis sout sépn rées par une latte plus étroite et en lenx pieres, que lion retire pour clégager le dentier (Fig. 32) L'upiculteur se selt y'urement fe cé dentier. L'ex. pé rience lui "rpiend 1 itr à bien alisposer les cudres. 
Partitjons - l'unr restreviudre à volonti la capacité de In ruche, M. Jullant iniplojir deux purtitions mobiles suspendues comme lin enitres et qui Hampluent cellx-ci h droite et gauche quand la rnclue nest pus pleinn. (tige. 333).

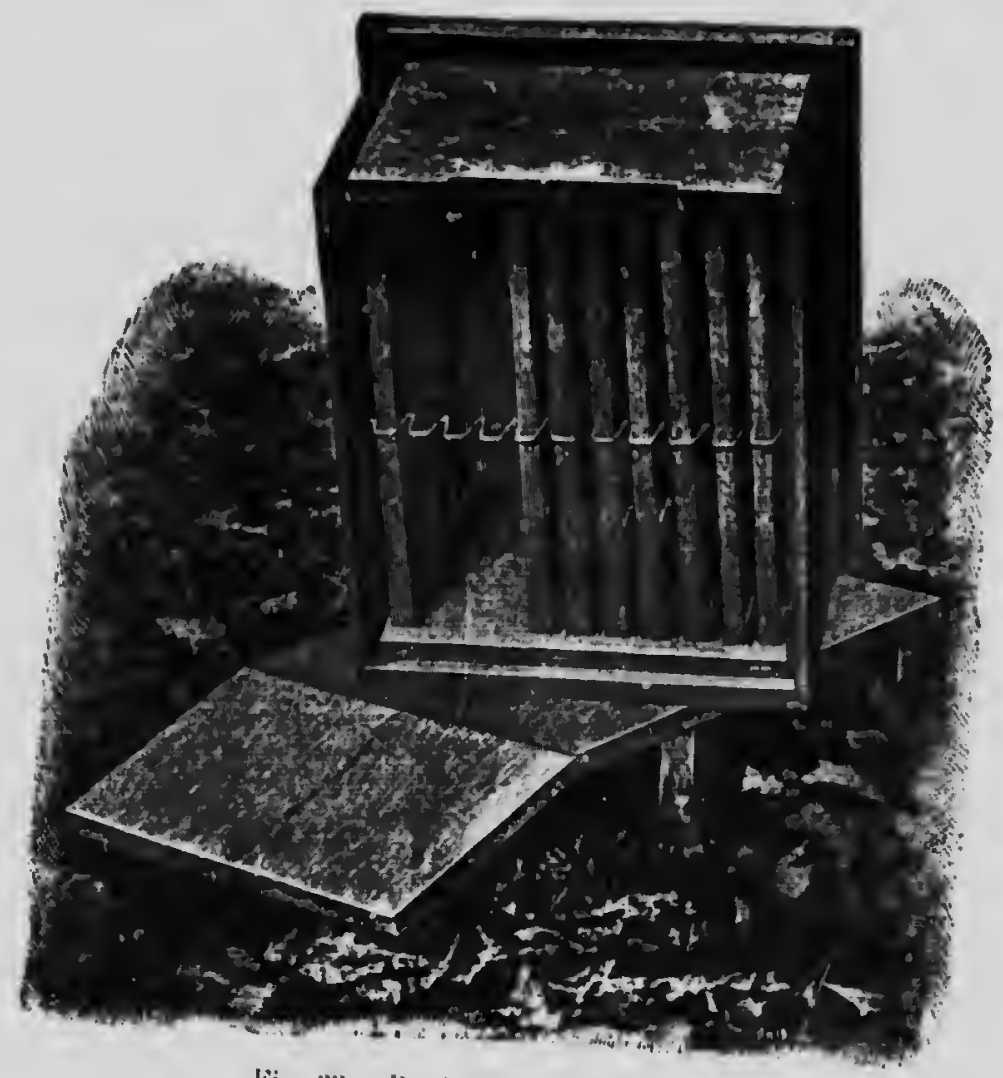

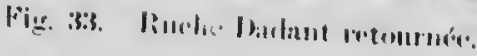

Ce sont des pliurducters an hois qui s'uluptent smr les

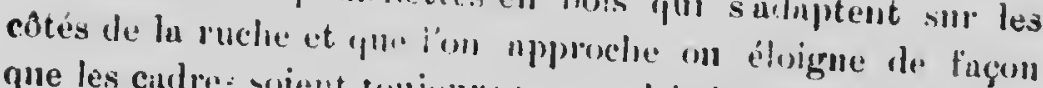

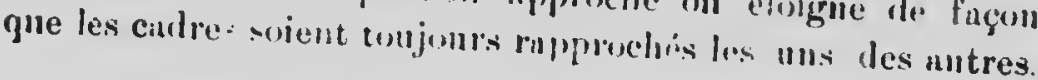

A menme proe l'on introduit de nouvenux cadres, on recule les partitions, puic on tinit par les enlever. (tigr. 34). 
Trou.de-vol. -- La passage dres uleilles ext ménngú duns In paroi de devnnt, an baw. C'est une onvertule do $3 / 8$ de pouces sur $\$$. Chacun la restreint a mn Inunière; M. Dadunt emploie tuut simplement un bloc de bois dur posé devant. On peut anssi employer le système indiqué par $M$. de Lonyens duns son traité, Flevage dos Abeillrs: Ine playue de inétal de la largeur de l'entrée est fixée par deux pitons nil-dessus de l'ouverture et leux autres land's, repliées nux extrémités, sont ingngées suns lin plnque et se inunouvrent horizuntulement. (In pent pratiquev luns ln plaque de métal deux fenteg en hinis dans lesqunilles passent les pitona, ce qui

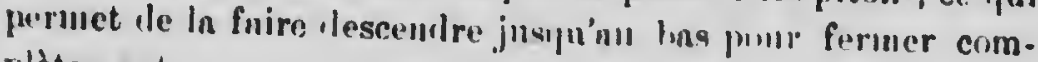
plètentent.

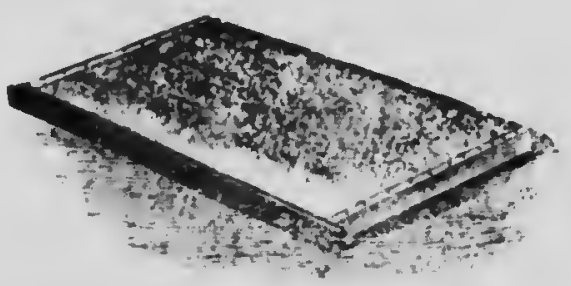

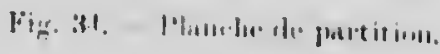

Couverture des cadres. .I. ()inlunt n en succinaive.

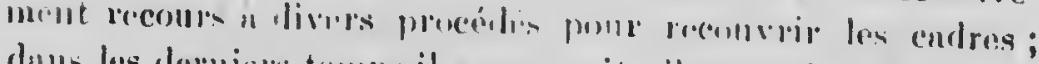

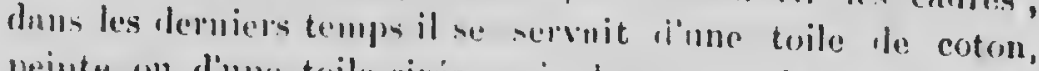

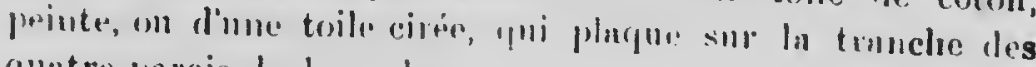
quatre parois de la ruche.

En la roulant sur allp-mên', on pent ne décoms rir de la ruche que ce qu'sl funt pun linpirition is fuire. I

Hausses ou boites de surplus, cadres des boites Ce sent des coni-ses smins foml ni comsercle que l'on fait de

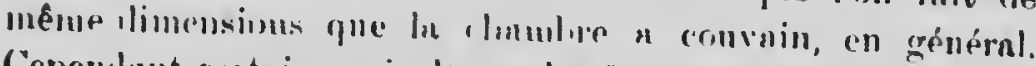
Cependant cestains npicultenrs les font somvent muins hau.

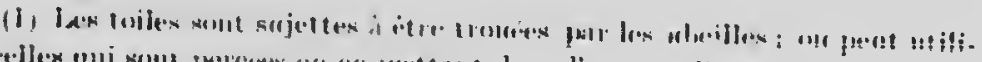

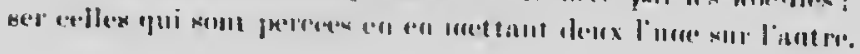




\section{MICROCOPY RESOLUTION TEST CHART}

(ANSI and ISO TEST CHART No. 2)
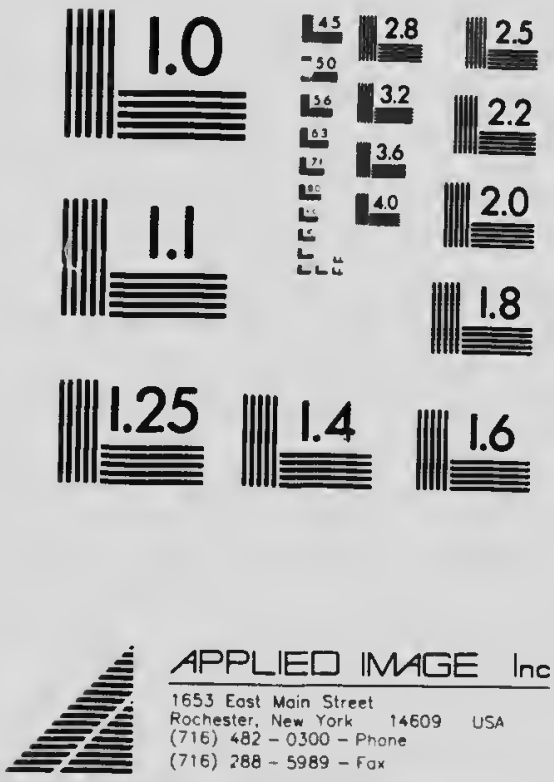
tes; mnis lmus co cos il fint so procurer lenx espèces do

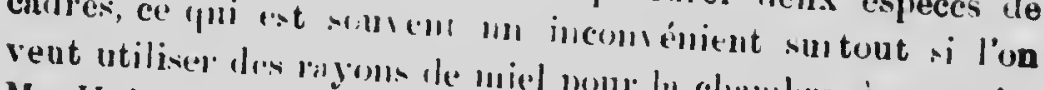

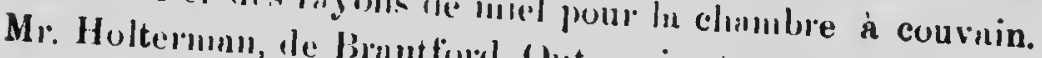

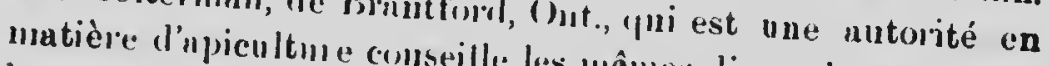
huusses que ponr les chimmlıpes des mênes dimensions pour les faire de miel en ruyms.

\section{Chapitea}

fuite de bois d'envirun 1 line de la rinche est une caisso formé de plunclies qui déburrlent épilisscur et lont le fond est Placéc sur la ruche, elle est siuportent untour de 2 a 3 lignes. ligne l'éprissenr, clouées tunt le tourée par des lattes de une

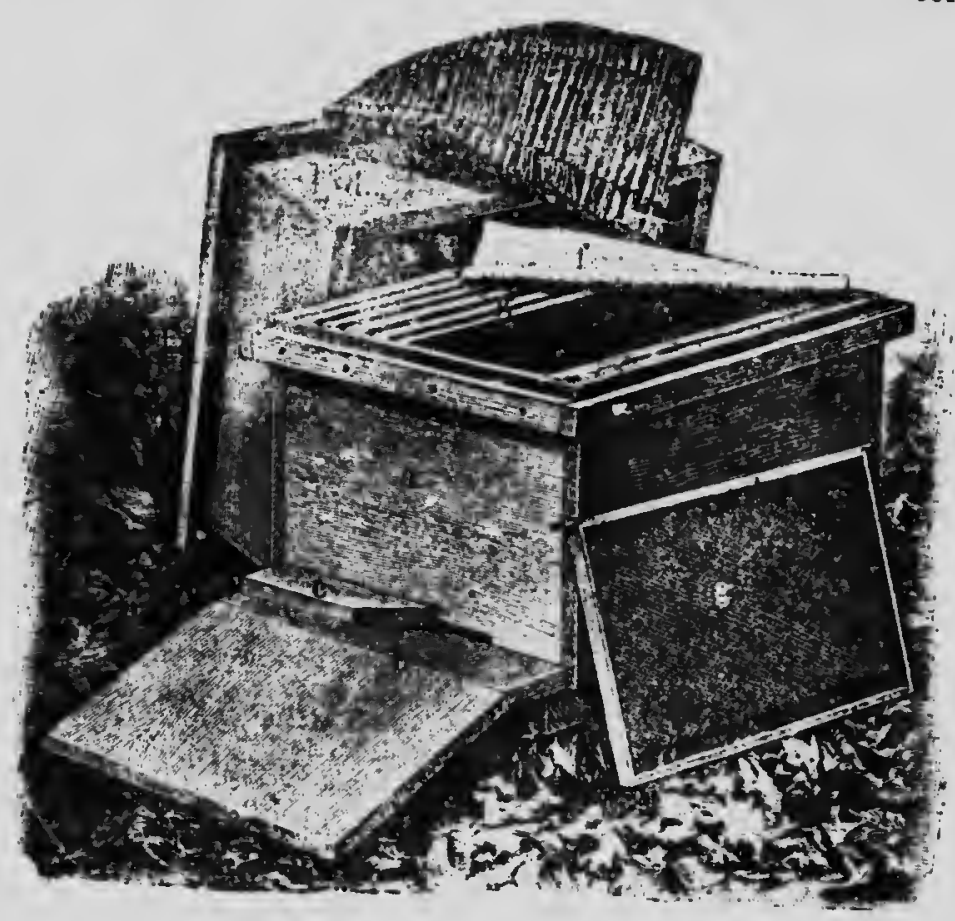

Fig. 3:- Ruche Darlant.

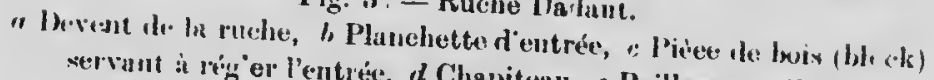
vervant at reg' er l'entréc, $d$ Chapitean, e Paillasson, , "Toill. peinte, yg Cadres gamis de rayo: s. 
et ì une hautəur telle ym. le courerch embite de 2 lignes envirour. Les tranchies dil convercle w te des lattes se rencontrent suivant un̄plun incliné an dehors, de frocun que l'eau qui dicoule le long du convercle ne sejjourne pas sur le bord des lattes.

Ln tig. 35 reprexente la reche Dadant telle que II. Dadnut l'employnit.

An lien de faice le dersus pint, lon donte nussi nu chapiteau la furmc d'un toit à deux versants dont la ligne de partage ent diuns le sells de lu lo grteel. De la ges rebords

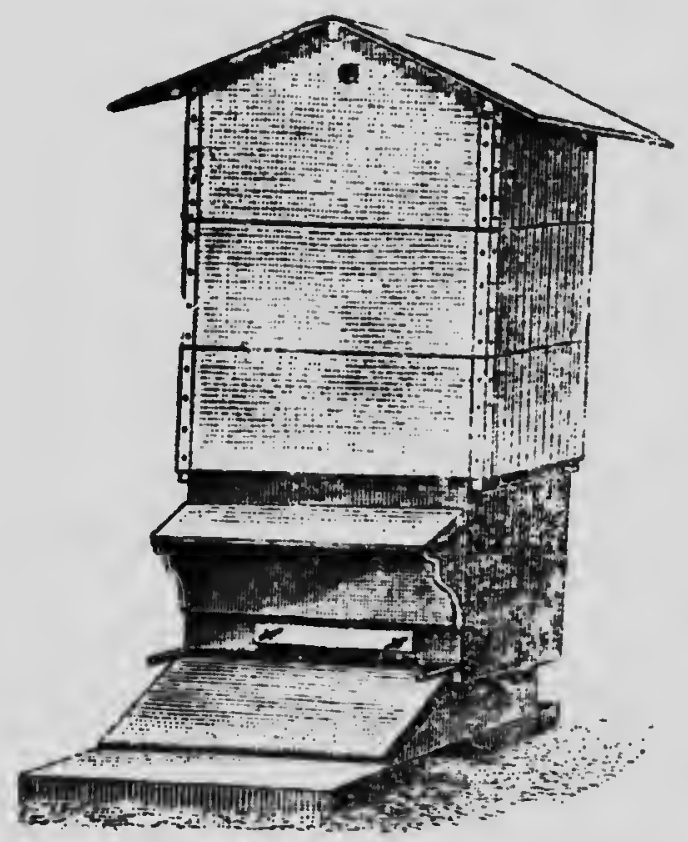

Fig. 3fi.--Ruclue ladint aree trois boites.

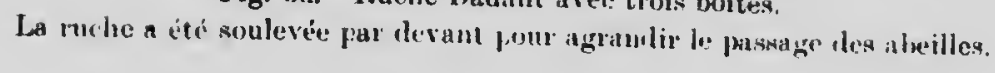

protègent In ruche et lui dom nent l'ispect de nos chnlets. On lui a aussi donné tomt simplement une forme bombée qui donne_d'excellents résultuts pour l'écoulement de l'enu. (fig.36) 


\section{('HA I'ITRE TIXIEME}

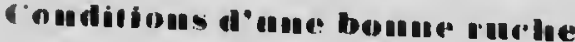

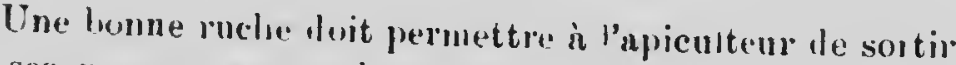

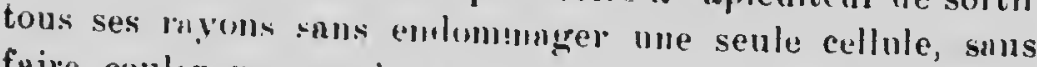
fuire coulcr me soule grontte de miel et surs exciter la colire des nbeilles.

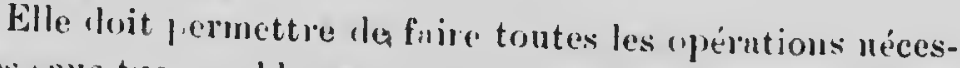
snires suts tuer on hlesser une senle ubeillo ; certuines ruches sont construites de tollo façon qu'en ne pout s'en servibsans détruire quelpues uleilles. Cette destruction, si petite soitella, angurente les ditticultés de lenr culture.

Elle doit protéger sutfisnmment la colonic contre les clualeurs et les fouils extrimes, contre les changrencnts soudains de tempéruture et contue les effets mulfnisnuts de l'humilité. J'intérienr d'une rnche doit atre stcen hiver et exempt en ét: ane chaleur trop forte ct suffocunte.

Elle doit épurror. nux uleilles tont tronjet inutile. Comme la snisun de la récolte ne dure sonisent que pell de temps, l'organisntion de la rucle doit fuciliter le plus possible le truvail des hutineuses. Jes rnches qui les forcent a traverser ave leur rhurge les layons couverts d'aleilles doivent être rejotées. An lieu re se fiayer un chemin d travers les groupes, les butineuses loivent avoir ln possibilité de se rendre dans lus greniers ì provisiuns san-, pour ainsi dire, passel sur les luyous.

Elle doit pouvoir s'ingranlir ou se rétıécir suivant la force plus on moins granle des populations.

Elle doit permettre l'employer ln cire grufréc et d'uti. 
liser tous les morcenux de bons myons l'uuvrières, au lieu de les fondre pour en fuire de la cire.

Elle doit empêcher la multiplication des mâles en fucilitant le remplacement des rayons à grandes cellules; une ruche contenant une trop grande quantité de rayons, bons seulement pour y loger du miel ou pour y élever des mâles, ne peut guère prospérer.

Sun plancher doit être attaché on mobile, ì volonté, tant pour augmenter la ventilation que pour frciliter a l'apiculteur, aprìs l'hiver, l'enlèvement des débris et des abeilles uortes. Sins cette précaution les cadnvres se putréfient sur le platenu et mettent en danger In santé iles abeilles. Fn ontre, quand celles-ci sont forcées de les truiner dehors, elles tombent parfois sur la neige, s'y engourdissent et $y$ meurent; car sonvent une aheille, volant au loin chargée l'un cndavre, ne pent s'en létnch"re et tous lenx tombent à terre.

Ancune partie de la ruche ne doit êtré plus lusse q' l'ouverture qui sert l'intrét aux ubeilles sii un nigg'igre -e principe, les abeilles ont a porter leurs mort et ?.inrs leluris en montant, avec gramle perte te temps et l'efforts Cette condition indispensuble n'est guère passible que lorsque les rayons uboutissent in l'eutrét re qu'on nomme, purr cette rnison, rayons froils. Toute inclinnison de arunt in l'arrière est impossiole quand ils sont placés parallèlement à l'entree, car les abeilles, qui construisent lenrs rayons alısolument perpendiculnires, ne les plucemi jas exuctencent dans les calres.

Elle doit permettre de nourrir les aheilles, en tr.mps frais comme en temps chand, qu inil elies en ont besoin.

Elle doit oftrir ln fincilité d'élargir, de diminuer et mêıne de fermer l'entrée, pour protéger la colonie contre les pillardes, et ces changements de l'entrée ne doivent pas forcer les abeilles à perire un temps précienx pour la retrouver. 
Elle aloit foumir la facilité de laisser entrer tout à coup un large cournut d'uir, atin que les uleilles soient excitées $k$ surtir pour décharger leurs iutestins, lors des journées chundes du commencement du printemps. Qunnd les abeil. les ne profitent pas de ces uccasions pon? sc vider, elles peuvent sonffrir et monrir d'une réclusion trop prolongée.

Elle doit permettre anx abeilles de pusser nisément et saus tâtunner dums les chambres du dessus, tout comme aussi à l'uir, à ln chmleur de pussej librement de la ruche da la huusse.

Chacunc des parties de toutes les ruches d'un rucher doivent être fubriquées assez exnctement pour pouvoir s'échanger d'une unche à l'autre. Par ce moyen, l'upiculteur peut fuire des échanges de rłyons, de courain, de rijel ou de pollen, su: iant que les circconstances le demandent.

In ruche loit permettre à l'apiculteur de récolter le miel sons la forme la plus belle la plus vendable et ln plus convenable, tant pour la qualité que pour la qunntité à récolter en tenips opportun.

Elle doit comvenir égnlement pour ln pratique de l'es. saimnge artificiel.

Elle doit permettre la multiplicntion de colonies apec certitude et rupidité; deux choses impossibles, si un conıte sur l'essaimnge naturel.

Elle doit donner la freilité de remplincer les reines chez les colonies orphelines.

Elle doit permettre à l'apiculteur de s'emparer de la reine, s'il le désire, pour la remplacer par une autre plus féconde, on p'us jeune, on d'une race meilleure.

Elle cicit permettre ḋ un seul npiculteur de soigner plnsieurs centaines de colonies dans différents ruclers, on pour differents propriétaires. Beaucoup de personncs auraisnt des ..eilles, si un rucher pouvnit être, comme un jurdin, 
soigné par un homme comprétont: umis persomne n'necepterait une phreille tinc.e mec les ruches in raym-tixus. Cumme les nbeilless essniment beancoup lans ces ruches, il pourrait être appelé daus diftérents endroits en mềne temps; puis si par accilent, quelques colonies devennient orplodines, il Jui sernit difficile l'y remélier.

'Tons les joints le ln ruclue doivent âtre nbsolument imperménbles et elle ne luit nvoir ni portes, ni volets snjets à se déjeter, se grontl.r. se rétrécir, on in mal fontionnel:

Lat ruche doit êtro protégése contro les dépréslations iles souris en hiver.

Elle loit pormettre de rassembler les rugons contenumt du miel dans la pluce oin il sern le mienx pon ln consonnmation rle l'hiver.

Elle doit permettre d'élargir et de diminurer à volunté l'espuce oi les abeilles mettent le miel de smprlus, sinns toucher en quoi que ce soit on clinnger l'uppartınent inférieur; sans cette fracilité, la prodluction l'une colonie pent être, en certaines saisons, irmulement amoin Iric.

Sin lanuse, on boite de snrplus, iojt itte anssi ruppruchée du couvinin ine possible.

'lout eu rénnissunt toutes les conlitions que nons arons émunérées, son prix, loit être nussi peu élevé que possil,le et sa forme simple, pour être à la portée de cenx qui lésirent se la prucurer on ln construire enx-mêmes.

Remnrquons qu'il y a des chuses lésirables que la ruche la plus conplète n'unra jamnis la prétention da lonner.

Elle ne pour ${ }^{n} \boldsymbol{h}$ jumnis promettre de bons résultuts à ceux qui sont trop ignorants ou trop peu lahorienx pour soigner leurs ubeilles. En apiculture, comine dans tonte autre occupation, un homıne eloit avant tout connuitre son affuire et ensuite se conformar à la vieille maxime que c'est le tra. vail qui enrichit. En un mot, pour réussir en t.piculture, il faut: "suvoir ce qu'il faut fuire et le fuire à propos." 
"Cunférences de Mr. K. F. Holterıau, de Bruntfori, Ont. devant les "piculteurs de Howick, Dundee, etc."

Ia meilleure ruchu n'est pas un talismun cupuble de rendre boune une contrie peu favoruble $\dot{x}$ ln récolte du miel; de inêue gau'elle ne peut donner ì l'apicultưr une bouno récolte, que la snison soit ou non furomhle; pus plus que telle vuriété de blé ne pent domer nu cultivatumr une récolte énoruse, quel que soit le sol ou la snison.

Elle ne pourin permettre a l'upiculteur use multiplicution rupille de ses ruchées en même temps que ln plus grunde récolte de miel. Ce sernit comne si un éleveur de volnilles s'imaginuit pouvoir fnire proluive à ses poules le plus grand

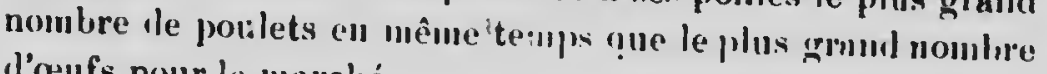
d'aufs pour le marché.

Peinture et revêtement des ruches. - On peint ses ruches de coulewrs clnires et vuriées pour niler les nbeilles à retrouver lenr lounicile. On peut nussi les greiull o h ynches et fuire varier seulement lu coulemr les planclucttes d'entrée. Les toiti recouverts de tôle sout peints en blunc.

L'expérience u enseigné qu'il est très utile de peiuclre aussi l'intérieur lu corps de ruche. Le bois non peint ì l'intérieur absorbe l'humidité produite par les alseilles et ne la rend pas si l'extérieur est peint. Pour l'extérieur, ln bonne ceruse délayée dans de l'huile de lin dégruissée est ln meilleure des peintures. A l'intérieur, l'ocre suftit avec l'huile; quelques apiculteurs emploient un vernis frit de propolis.

Isa peinture est presque une nécessité pour les ruches exposées aux intempéries, ì moins qu'elles ne soient revêtues de puille.

Nous devons conseiller de n'employer comme peinture que des couleurs claires et de les varier. Jes couleurs foncées absorbent trop de chaleur tet peuvent, en été, occasionner des accidents. La variété des couleurs nide les abeilles a 
retrouver lenrs moles; alle est indispensulve si les ruches sont très rilpirosehées. Cependant, on pent, comme nou., l'avous vin, se contenter de peindre de coulenirs diffëentes les planchettes des entrées; cette variété suffit générolentent pon' guider less nheilles.

II est indispensable aussi que chayue ruche porte III numéro peint en gros chiffres: pour qu'on puisse le lire de bi 1 . On le place bien en vue. (fig. 37. .

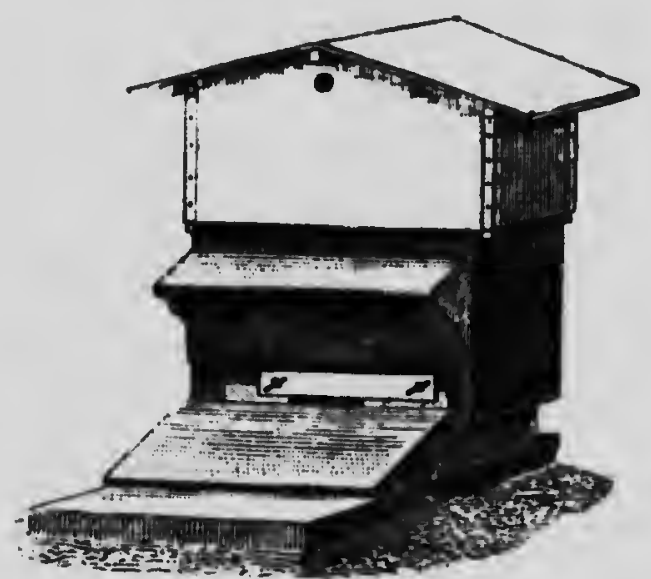

Fig. 37. - Rurhe Dardant, an primempr.

Si les parois : ont frites de plusieurs pièces, l'humilité est snjette a s'introduire dans les joints. Il y a des fabricants qui revètent le corps de ruche de carton peint sur les deux fuces, en recouvrant les angles l'équerres en tôle légère.

Visite et ventilation des ruches.-Pour exmminer un seul cadre, il suffit ciécarter un peu les deux voisins, en haut, ce qui permet de le sortir facilement.

Po.r visiter toute la ruche, on déplace d'un cran la partition et successivement chaque cadre, ce qui évite de revenir en arrière. Si la place manque, on eniève une 


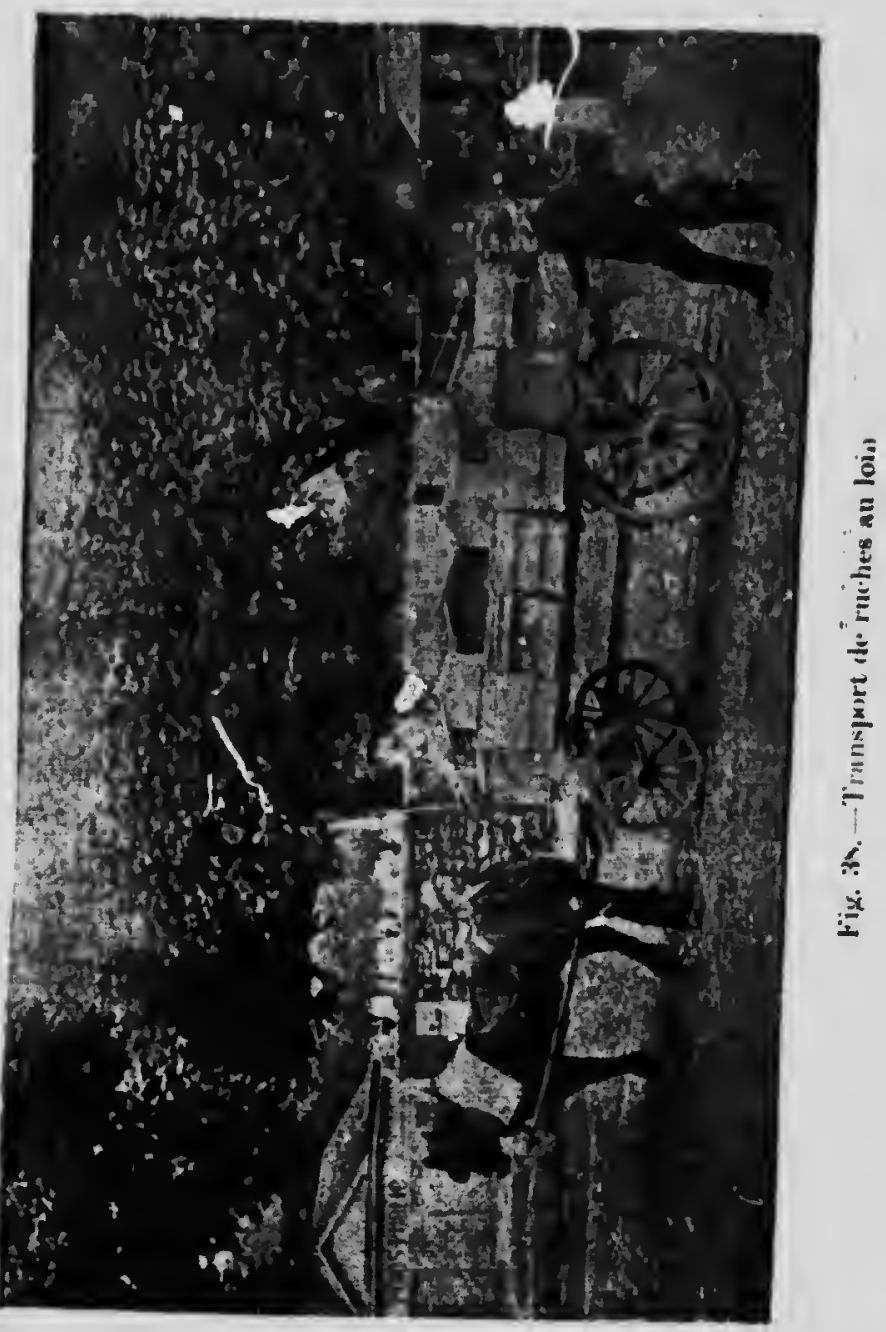




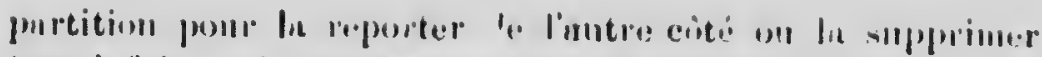

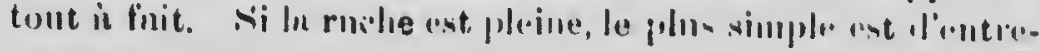

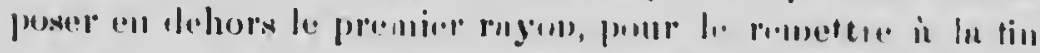

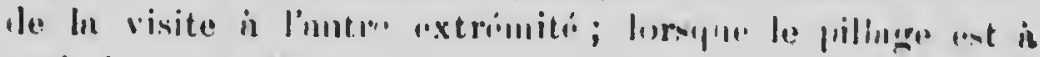
eruin Ire, all enferme ce myon dinus h lonite de trunspurt.

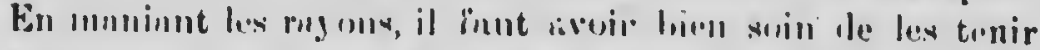

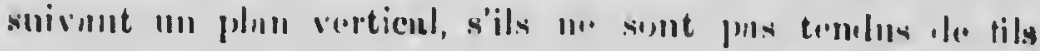

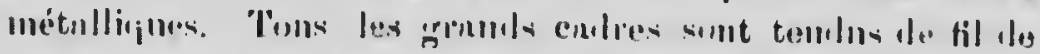

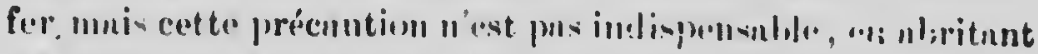

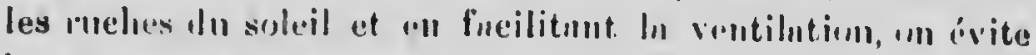
les mpenres des myons.

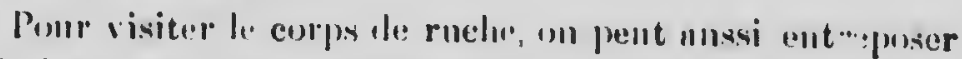

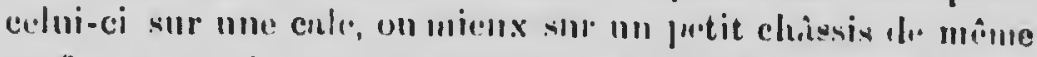

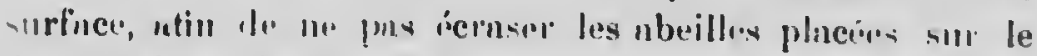

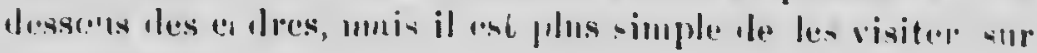
linur pluteaur.

Maulement des Abcilles, précautions à prendro

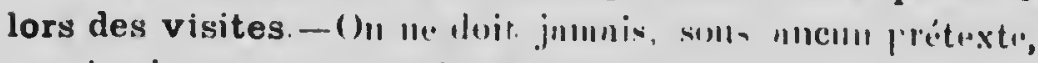

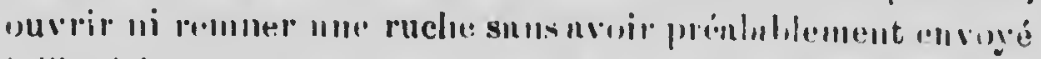

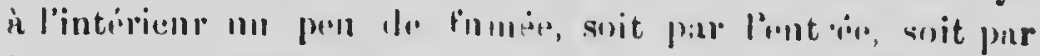

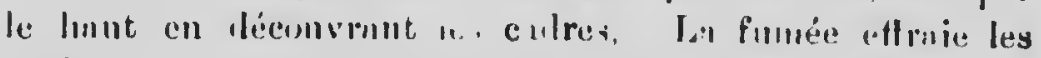

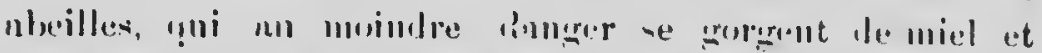
sont en-nite moins dispenser is pirinere : alle ost sans reffet sur les ruclies sums provisioms. Apré, nvidir enfume, on attend une lemi-minute nvant de procédei ì h visite pour laisser nux nleilles fe temp's d'ulisorler eln miel. Si les opérations se prolongent, on envoie de nourenu un pen de fumee pur le linut, ntin le refouler les nlieilles entie lex ringuns.

On les culme nussi en aspergennt les rayons de quelques gonttes d'enı sncré; c'eat nne ressonrce, lorsqu'il n'y n pas le miel lans la ruche.

Si l'on n à chercher ln reine, on enfune par l'entrée et très motérément.

Ayos soin qne tons vos mouvements mintour les ruches 
soieat tommpilles at lents. No iblesse\% ni ne presse\% jamnis tue seule ulwille, seg phintes exciternient lu colère des nutres. Si vous runs póncitre\% bien des conscils yue nous rous dommon - ct si vis les metter scrupuleusement ell pru-

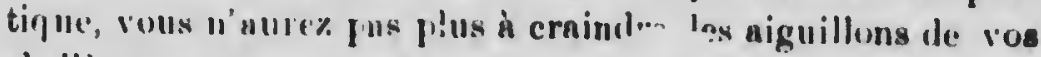
nheilles que volls un craigne\% les cornes de votre vucle do pridilection an les solotis de votre cheval favori.

Lenfuroir anficalu, (tig. 39 ), wst l'urme déf(nsive

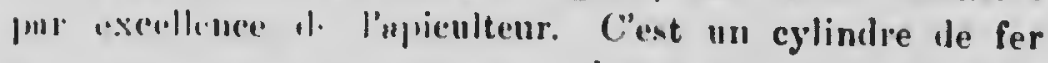

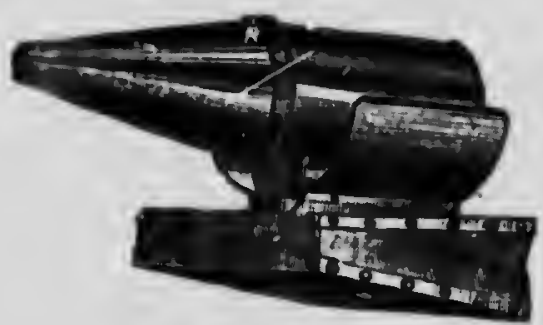

Fig. 3:1. Bofummir. linttu servant de foyer et monté sil' un petit souftlet in ressort. I.e couvercle, de follue ullongee, lonne passnge in ln funéc. L'enfumoir nII repos loit être Juns une position verticale pour rester allumé. On y liptle du bois pourri, le champignon du hêtre, de la tourbe, ou bien des chiftons ou du gros pupier gris grossièrement enıoulé. S'il est bien annlitionne, er sout ces deux lerniers combustibles qui indent le phis lomigtemps suns reehuran.

Précautions contre les piqûres. - Il funt Muili les monvements donx, ne pas fuire de grmuls geston ni pmer nsec In main l'uleille qui numonce de mnuraises intentions: In incilleure défense ent l'immolilité et la fumée.

En cos de pigûre, enleve\% pomptement le dard.

Beuncoup d'upieultenrs opèrent ì visuge déentivert, suf ou moment lu pré!èement lu miel après lia réeulte, inais le conimengant ferm lien de se protéger In face et le cou, ntin de conserver sou snng-froicl. La weilleure protection est un voile fait de tulle noir ì large trous. On en prenl un morceall de 18 a 20 pouces sur une verge $\frac{1}{\text {, d dont on coud }}$ 


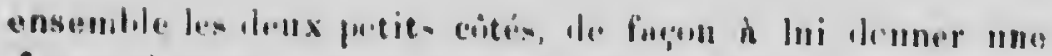

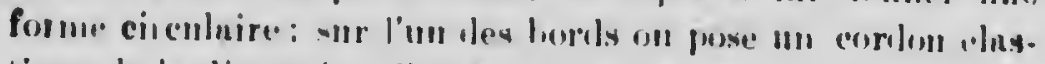
tivue de la diminsions al'un fourl le clinpenus.

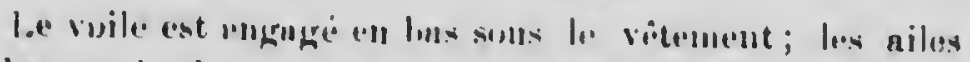

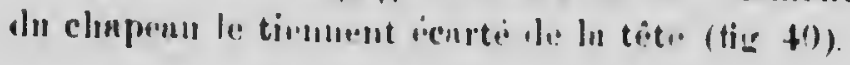

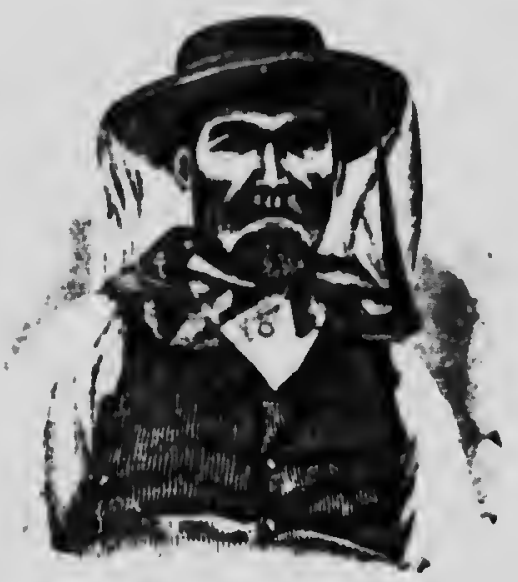

Fio. HII. - Vovil.

(1) jient protingere ses muins m moyen ale gunts

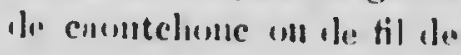
clumere tprusoier, muis cela

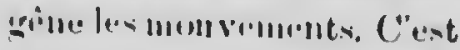
strtomt mas proignets, a

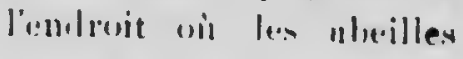
preseres surt lea umins snus mussuniare intention rencontrent le vitement. que

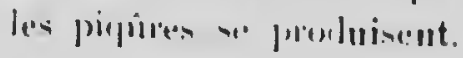

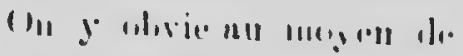

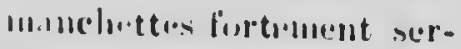

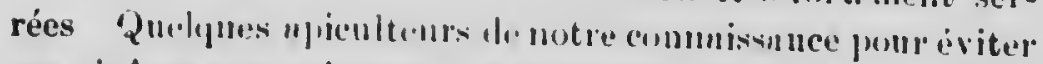

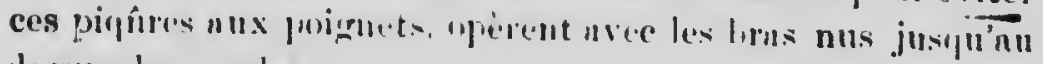
dessus les condes.

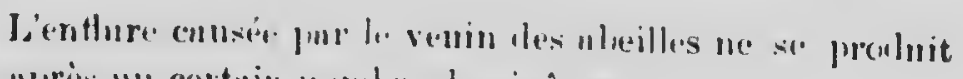
plus "près un cortuin momline le piquines.

Une prenière pingur. en attire l'nutres; l'odeur du venin irrite les nheilles.

Quanl les ubeilles posées sur le sommet des cudres se mettent à fuire le petits saut, avec les niles écartées, c'est le noment de redonbler nvec la fumée pour les refouler entre les rnyous; sans cette précaution, clles devienrlraient inéchantes.

Les abeilles se moutrent agressives lorsque, la ruche étnnt ouverte et lu visite se prolongeant, tles pillardes pro- 
vennut des rucles voisines commencent in s'y introduire; la fumée perl alors sun effet. Dans ce cas, le mienx est de remettre ln fin des opérutions ì un antre moment.

Lorsqu'il y a récolte, c'est le millien du jour qu'on choisit pour fuire les visites, [mec que les vieilles abeilles, qui sont les moins donces, sont dhors; an contrnire, lorsqu'il n'y n pas de miellée, il convient, nfin d'éviter l'inconvénient du pillage, de visiter les mohes ì plafund molile de prefirenee le mutin on le soir an sinon de fuire l'inspection lestement.

La farine employée comme pacificateur. - En sinupondrant les abeilles, on les' r'ml momentanément inoffensives; crtte propriéti de lin farme peut être ntilisée dans certnines upérations (trolles que les rémnions et remplacement: de l.imes) connse nons l'mon: v'u.

Apifuge. - Un Anglnis, II. (Himshaw, n emmposé un

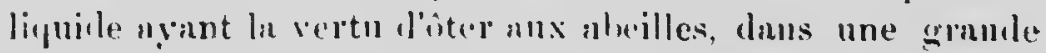
mesmar, la linpmition de pigner. ()n s'en enduit légèrement les mains (il ent volitil) et son onlenr, colle du winterereren,

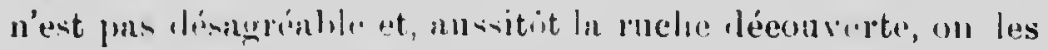
étend un-dlessuls comme proll lit magnetiser. Le's ubeilles en

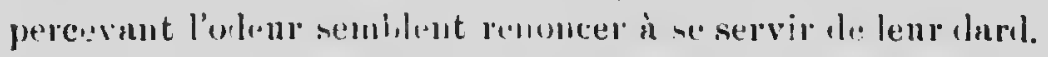
Nous arous fuit using ale cett. emupesition arec succis pour prélever le miel lans des rochées de manrois caractèce; elle n'est phs infuillible, mais soll emploi, acempugne de monvements donx, excrecertainement me netion sédntive sul les

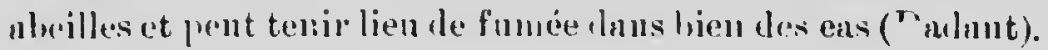

\section{VEXTII,ELSIS.}

Si, par un jour tle grande chalenr, on exumine une colonie populense, on munryuera une quantité d'aleilles sur le plancher nyant lenr tête tournéc du côté de l'entrée, leur abdomen légèrement élevé et leurs niics frisant ales monveinents si rupiles qu'on ne peut prosque pas mienx les roir 
qu'on ue diatingue les rais d'une rone, yuan I elle tomme nvec une gruncle rapilité autour de son axe.

On pourra con-tutar qu'il sort le la rucle un vif courant d'air st si on suspent mi morcenu de ilucet à l'rntrée on remnrquern que pendant qu'un. le ses purties est reponssée, l'antre cost nttirée pur l'entrée. Ponrquoi ces aleilles sontelles nlsworlées par leur vceupation l'éventer. nu point qu'elles ne soccupeut mullement de celles qui ne cessent ilentrer et de surtir et qui sont cepenlant en graul nomlire: Que signitie ce louble connut a'nir? Huher uous a dunne une explication sutisfuisante le ce curieux phénomène. Les abeilles qui font vilorer leurs ailes d'un mourement si rapide

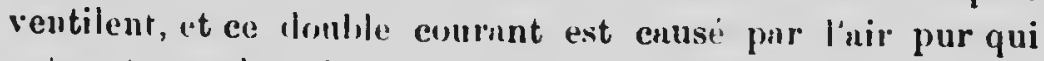
entre et remplace le munvais air qui sort. Par une sélie d'experiences intéressuntes, Huber a reconnn que l'air d'une ruche très peuplée est presque aussi pur que l'uir le l'utmosphère eluvironunte. On comprend que, comme l'entrée d'une ruche est somv zut très petite, son air ne peut être renouvelé que par les moyens artiticiels.

C'est en étuhlissant un doulle cournnt d'air que les abeilles éventent leur habitation í population serrée. Une brule de ventileuses se placent en ledans et en dehors de la ruche avec leurs têtes tournées ln côté de l'entrée, et pur le mouvement rnpide le leurs ailes un rupide conrant d'air est lancé hors de la ruche, tandis qu'un cournnt égal y est attiré. Comme ce service important exige un trarail futignant, les ouvrières lassées sont remplacées par de nouvelles recrues. Si on ponvinit voir l'intérieur le la ruche, on y trouverait des ventilenses répanlues çn et là et engngées ì cet emploi lahorieux. Si on rétrécit l'entrée re la ruchre, leur nombre est augmenté russitît, tant en dedans qu'en lehors et, si elle est fermée entièrement, li ch leur et l'air impur nugmentant, la population entière esmiera de renouveler l'air en battant des ailes et mourra bientôt de suffocation, si on ne lui rend pas la liberté. 
Des expériences fuites nvec soin ont montré que l'air pur est nécessaire non seulement aux alreilles, pour leur respiration, mais nux áuf et aux larves en léveloppement, un tin résenu de vaisseunx nériens enveloppant les oufs et les nymphes étant enfermées par des opercules porcés de petits truus $\dot{x}$ uir.

En liver, si les abeilles sunt placées luns un endroit obscur qui ne soit ni trop chnuld ni trop froil, elles sont presque endornies et n'ont besuin que de peu d'nir; mais, unême lans ces circonstunces, elles ne peurent absolument s'en p ısser et si elles sont réveilléos, soit par un changement atmosphérique, soit parce snom les a tronblées de quelque façon, on peut entendre lans lenr ruche un long hourionnement ; elles ont alors hesoin de presifue antunt dinir qu'en temps chund.

Si on trouble henuconp les abeilles, comme pomr les transporter, par exemple, il est pen prulent, surtont en temps cland, de les enfermer, à moins qu'on ne laisse l'air entrer librement dans lemrs mches : et même, ì moins que cet air ne soit nduis au-lessus comme uu bas de la masse des abeilles, les ventilatenrs sont hientît bouchés par les cadavres et la colonie périt. Les nbeilles quand clles sont renfermées s'échnuffent beaucomp, an point que sourent leurs rayons se fondent; elles léveloppent duns la ruche nne telle chaleur que l'humidité contenue dans les laries qui vienment de périr, lans leur nourriture et dans le miel, s'éva pore. La buée ain-i forınée mouille les abeilles autant que si on les eât trempées lans de l'eau et elles périssent. Si on leur donne le l'air avaut que tontes n'aient été asphyxiées, celles qui restent semblent avoir vieilli, tant leurs corps sont devenus noirs et luisants. Elles ont, en outre, l'abdomen distenlu par le miel dont elies se sont empressées de se gorger anssitôt qu'elles se sont vues enfermées, comme elles le font dès qu'elles se croient en langer, et elles se vident 
comme qunml eiles ont la liarrhée. Quelynes in-tunts suffisent pour proluire l'aussi fatals résultats.

Les débntants en apiculture ne se doutent guère du danger d'afficissement auquel sont exposés les ruyous de miel durant les chaleurs de l'été. Dans l'été de 18ī7, nous avous ru, dit Dudant, des rangs entiers de ruches d'un rucher inportant, qui étaient exposées aux rayons du soleil de nicli, tomber en pâte presque au mêıne moinent, cuusant une perte de plusieurs centaines le francs, par inanque d'une ventilation suffisante, qui nvait forcé les abeilles à se rasseinbler all-devant et sur la ruche. Quand un rayon se ramollit pur lu chaleur au point de tomber, le iniel se répand sur le plateau, sort p.ur l'entrée, engluant les abeilles et em. pêchunt toute ventilation; alors les rayons, qui jusque-là avaient résisté, s’amollissent ì leur tour, écrasant couvain, reine, ouvrières. C'est une entière destruction. Depuis l'aeeilent que nous venons de relater, nous avons pris la précrution. en été, le soulever nos ruehes de l'avant et d'ourrir un esurant l'air eomme nous le inontrons dans les caltes placées sons le platenu de la higare 33.

En Canula, nous ne sımmes gruère exposés à ees chalenrs torrides. Toutefois s'il en snrvenuit, $i$ ! serait anssi siuple de faire de l'ombre à l'aide de brunches auprès des ruehes trop "xpusées nnx nrideurs du soleil. 


\section{CHAPITRE SEPTIEME}

\section{J.A CULF. GAUELEF:}

Linvention et l'intraluction le la cire gaufréc* dans l'emploi des cndres mobiles a fait fuire un granı pas au progrès de l'apiculture. La principale difficulté dans l'comploi des ruches $\dot{t}$ calres mobiles était d'obtenir toujours des rayons droits. A la vérité, le bisenu de la planchette suppó. rieure du cadrc réussissnit très souvent ; cependant quelquefois les abeilles s'écnrtaicnt de ce guide et nttachajent le rayon au cadre suivant, et si on n'y portsit promptement remède les ruyons devenaient aussi difficiles à sortir que ceux d'une ruche à rnyons tixes. Un cadre quelque peu hors dc place étnit pour les ubeillcs un encouragement à attrcher deux cadres enscmble. Les rnyo' is qui étaient bien construits dans les cadres étuient eux-mĉmes parfois ondulenx ou bombés pur places et rendus ain $i$ impossibles r̀ être donnís à l'inutres ruches.

Une antre difficulté dins ln réussite était la prodnction des ruyonsile miles. l'on olitemait des coloniıs qui ont un quart de leurs rayons en cellules de mâles. Duns ces ruches, le nombre de bourlons qui pouvinent être élevés nurait été suffisant pour consommer tout le surplus. Certainement la mobilité des culres permettnit l'enlèvement de ces rayons, mais il était difficile de se procurer des rayons d'ouvrières droits et propres pour les remplacer, cur si on retrunche simplement les rayons de inâles dune ruche, los nbeilles les

\footnotetext{
" Qu'on nomme en anglais comb. fonmlution (fondation de rayon).
} 
remplacent le plus souvent par d'antres dı même geurr. ()n a obvié à cet inconvénicnt par l'emploi de la cire gnnfíre.

De lons rayous l'ourrières lien drojits ct qui ne sont pas trop âgés sunt le meillenr cupital de l'upicultenr.

In consilération de ces points importunts et le ln lépense que fout les alseilles ponr construive lanis rayons $n$ penrlant longtemps nttiré l'attention des apiculteurs allemourls sur In possibilite de fubringer la luse on fondution des inyous.

En 1857, Jem Moluing inventa une presse pour fuire des gaufres cle cire sur leoquelles les ruliments ines cellules étnient imprimes. Ceux-la senlement qui ont ell in surmonter les obstucles de cette industric peurent se fuire une ilée le ln volonté et le ln persévérnnce qui lui furent nécessuires prour réussir. Ln cire gnuf rée finite nlore par lui étruit loin d'éguler ce qui se fuit muintenant; les saillies des collules étuir nt trop rulimentuires, l'impression leur manquait parfois et les nlneilles y construicaient des cellules de mâles an lieu de cellules d'ouvières; mais ce travail imparfait étnit le début d'une indnstrie qui a offert un avantuge immense anx upicnltenrs ('t yni s'est répnnlue partont oì on cultive les nbeilies.

Un antre npiculteur, le ln Suisse, Pierre Jacoh, a numelioré In presse de Mthring, et elr 1865, M. H. Steele, de Jersey-City, importu de sn cire ginfrée nux litats-Unis. Cette cire ganfréc fut essnyée par II. J.I. Hıiburd, qui en fit l'éloge.

Ln première cire granfrée finite en Amérique fut fulriquée en 1875 par m. Allenmml, M. F. Weiss, tiès probablement arec une mnchine importée. M A. I. Ront, auquel on doit la popularisation le cette invention lans le monde entier, tit fubriguer nue lng une une à cylinclres, "n février 1876. par un ouvrier liabile, M. A. Wushbmue. Lepnis lors, il a vendu des centnines de ces unchines. 


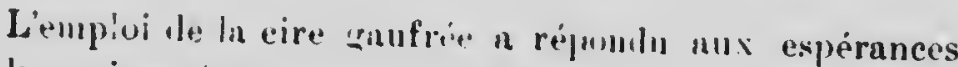
les plus exigenutes.

1" lont lanyon lati sur la cire gnufrée est nussi froit qu'une planche et peut pienlre la pluce l'un autse dans toute ruclue sums nucure peini.

2: Les rnyms hâti sur le ln cire gaufrée sont à cellules d'surrières exclusivement ; ì l'exception cepentant de petites pluces vir In cire gunf rée a ju s'ullongè pur le poils.

3 'Tute In cire produite mor ies niseilles et recueillie par l'apicultenr lans les gruttuges, daus les vieux rayous ou dnus les opercules, est rendue nux nheilles en cire ganfrée, nu lieu l'itre remlue pónr In vinleur de ln cire, qui est plusieurs fosis inférieme un prix çu'elle a conté. Le déboursé pour la cire gunfrée n'est pus très graul, surtont si nous consillérous que ce capitul n'est pus entièr sment perilu, puisque ln rire contemne lnus le royou représente au moins

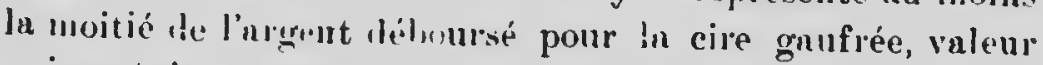
qui pist itre rotondép in cire uprès des nnnées l'usage, suns qure celle-ci suit plus murvaise qu'an début.

in cirregnufrée ì fonl plat a ln réputation dêtre la plus légère at l plus rágulière; son défuut est le fond plat des cellules, qui rend sa fubrication plus facile, muis n'est pas du gont les nheilles, qui unt à refuire sn buse avant de

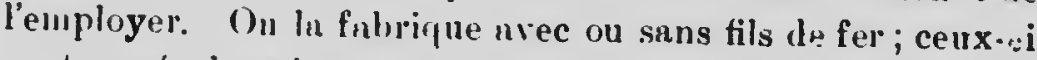
sont noýs duns ln cire et airlent à l'attacher dans les cadres.

On emplosir mux lituts-Unis difiérentes sortes de machines.

In machine Pe liam finit de la cire gaufrée dont la base des cellules a deux ciotés au lifeu de trois. Cette nachine, qui a l'aruntuge d'être très bon marché, est d'une manipulation plus facile que les autres.

Ln presse de Guven fuit de la cire gaufrée par un procéclé semblable à celui des anciennes presses européennes; 
elle a reçu beaucoup de louanges de la part de certains apiculteurs. Comme c'est une inuchine très facile ì inanouvrer, beaucoup de ceux qui n'uvaient pu faire fonctionner les autres, réussirent uvec celle-là. Un autre avantage qu'elle présente, c'est qu'on peut insérer des tils de fer duns la cire gaufrée en l'imprimaut. Mais cette presse a l'inconvénieut de laisser aux feuilles tontes les irrégulurités de la trempe, tandis que duns les machines à cylindres, ces irrégularités dispa raissent dans le laminuge. Pur conséquent la cire gnu f rée pressée n'est jumnis nussi régulière jue celle faite par des roulenux.

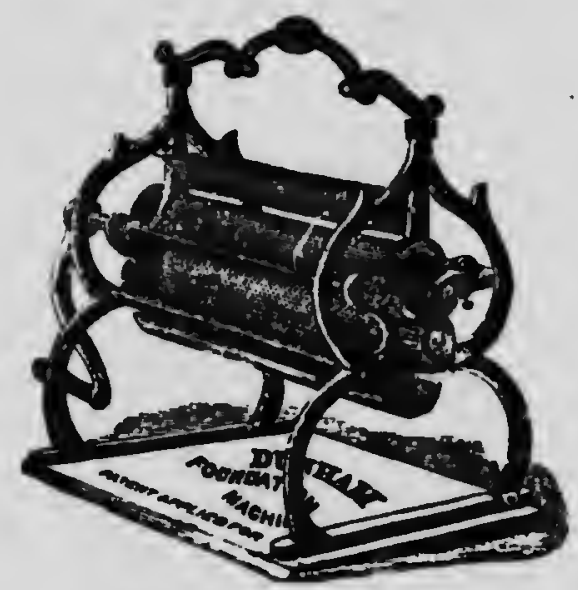

Fìg. f1. Machine Duuham.

Les machines ì cylindres le Root, qu. sont les plus convenubles, ont été nuéliorées de diverses f „ı̧ons : par Olı, du Michigan; par Ilme. Dunh.m, lu Wisconsin (fig. 41) et par J. Vandervort, de la Pensyl ranie (fig. 42).

La cire qu'on emploie pour les feuilles minces de; boîtes de surplus est d'une qunlité de choix. Celle des opercules est la ineilleure pour cet objet. Néanmoins, soit que la cire doive être employée jour les boites de surplus, soit qu'elle doive servir pour la chanbre à couvaiu, il ful la nettoyer parfaitement, en la chanffant lentement dans des vaisseaux 
évusés dont on pent ln retiver fneilennent. Si les corps étrumgers restnient luns ln cire, elle aurait moins de consistunce et s'ullongernit plus niséurut. Lo nettoyage au moyen d'ucirles, tel qu'il est employed par les blunchisseurs de cire ne convient pus, cur les ulveilles out do ln répuguance pour toute odeur désugrénble.

On ne loit fus emploger nutre chose que de la cire pure pour fuire ln cire gunfrée. In parnftine, la cérésine et d'autres unntières ont été issnyéres avec dies résultuts ilésastreux; suns compiter que ces sulist unces se liquéfient a un degré plus lins que celui de lu cire* et tumlient en pâte dans

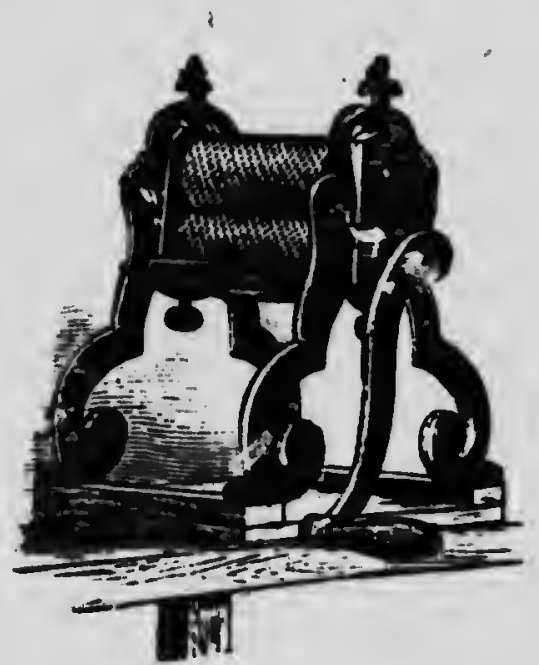

Fig. 42.-Marhine Vandurvort.

la ruche; les nbeilles reconmissent ln trompiric et montrent une préférence biell positive pour la cire purre.

Ln falsification la plus employée pour la cire est l'addition de suif. Heureusement, elle peut être facilement recon. nue par l'aspect terne des pailla. L'odeur de suif est aussi

- Ia paraffine entre en fusion a 1100 Farenheit environ, la cire d'abeilles 1400 environ. 
fincile in reconmuitre, si :m sent les fingunents nusitit quim les $n$ lirisis.s.

Les machines employées pour tuire de la cire gnnul rie mince ne srmt pas les mêmes que celles prour ln eice destinée a la clumbre à consinin. Cette dernièro, si un lu frisuit sur lane machine in impressions légères, sernit trop finils pour supporter le poils des abeilles dans mu ruyon in convinin de grmuleur ordimaire et ne contienlrnit pas $1258 \%$ de circ pour que les . hilles puissent nchever le ruyon; c'est en eftiet un fait ret.arpunble que les ah illes anincissent le myon inufué et qu'elles le font considéru. lement plus profond nu unyen de su cire. Qunnd il a été finit à fond mince et ì côtés cipnis, les nbeilles all allongent plus promptement les ce'lules.

D'un untre coté las royons ponr le miel le snrplus doivent itre nnssi leigers que lit unchine peut les finire, ntin d'éviter cr ' (1n'on nppell. l'arrête intérienre ou mur centrul, qu'on remurpue dnus le myon de miel qui " été construit sur de la eirr gaufrée trop épaisse. Si on nemployé la sorte de fondution convonalile, mn connaissenr te miel en ruyon hésiternit is déclarer qur la hass du rayon est naturelle ou artificielle

Presque toutes les sections de miel en rayon qu'on

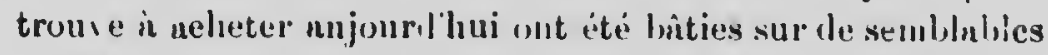
fondations les alume- les plus délicates et les plus dégoûtées n'y font uncmue oljection, et si elles visitent une mmufincture de cire gaufrée lien tenne, elles en tronvernt les fenilles minees npétissuntes. (Dulunt).

On ne peut cependant affirmer que dans la pratipue on snit nussi méticule!nx. L'on est arrivé à faire de la cire gaufréte qui lonne sutisfinction et ponr les calres des linusses et pour ceux in cournin tont en réservint les feuilles minces pour le micl en sections.

Pour préparer les fenilles de cire ine l'on loit passer entre les cylinilies, l'on se sert de planchettes we hois tendre bien uni, smn.; neuils ni défnuts, l'environ 5 lignes 
d'épnisseur. Ces plancliettes sout placées Inus un buin d'enu tiède, puis essliybes avec une épunge et trempées deux ou trois fois dans de la cire fondue maintenue au degré le plus bas unquel clle puisse rester liquide. In pai tie la plus basso de la planchettc est ensuite plongée dinur ie l'eau froide ; Ia planchette est tournée le liaut on has et le côté par lequel on la tennit est plongé h son tour et traité le mêne. C'ès opérntions sonf sufficantes pour de la cire gnufrée unince; on les recummence qunnel on veut nugmenter l'épaisseur. Apress que la planchette $n$ étu plongée dans l'eau pour ref roidir; on In sort, on coupe In cire lle ses trunches nvec un contean +t on ell pèle les deux feuilles. Si on I: employé ln cire trop chnule, les feuilles se fendent Pour obtenir un truvil plus rilpile, il faut avoir, pour les plongeurs, un local priparé exprès et muni d'un pluncher de fer-blunc, ou de zinc, ${ }_{1}$ mar recevoir les gouttes de cire et l'enu.

La figure 43 montre une tnble à lmminer. Ias fenilles de cire, qu'on a dî mettre refroidir pemlant qui iplues jours dans une cove sèche et froiche, sont ramollies duns un linssin d'enu churle urant le les passer entre les cyliniles ; ceux-ci sont rendus lisses et glissnnts nvec le l'umidon on de forte eau de snvon.

Quand on emploic l'ean ile savon, il est très importunt que les feuilles soient tellement pressées entre les cylindres yqu olles en sortent absolument sèches; cette pression fnit ıles feuilles plus régulières. La cire gaufrée, à inesure qu'elle sort. les rouleaux, est empilée, puis on place unu douzaine de fellilles ou invantage sur un bloc le bnis dur; on pose un patron de planche sur cette petite pile et on ln rogne de la limension voulie, au moyen d'un coutenu ì lame mince qu'on a trempée dans de l'eau de savon. Les bords sont ensuite rognés, et les rognures, ainsi que les feuilles non réussies, sont refonllues pour être employées plus tard.

La fabrication de la cire gaufrée, qui au commencement semblait devoir être entreprise par tous les apiculteurs, est 


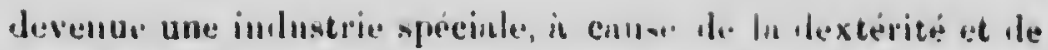

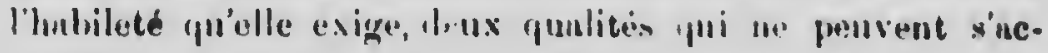

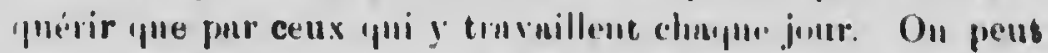

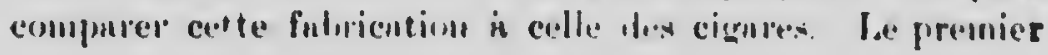

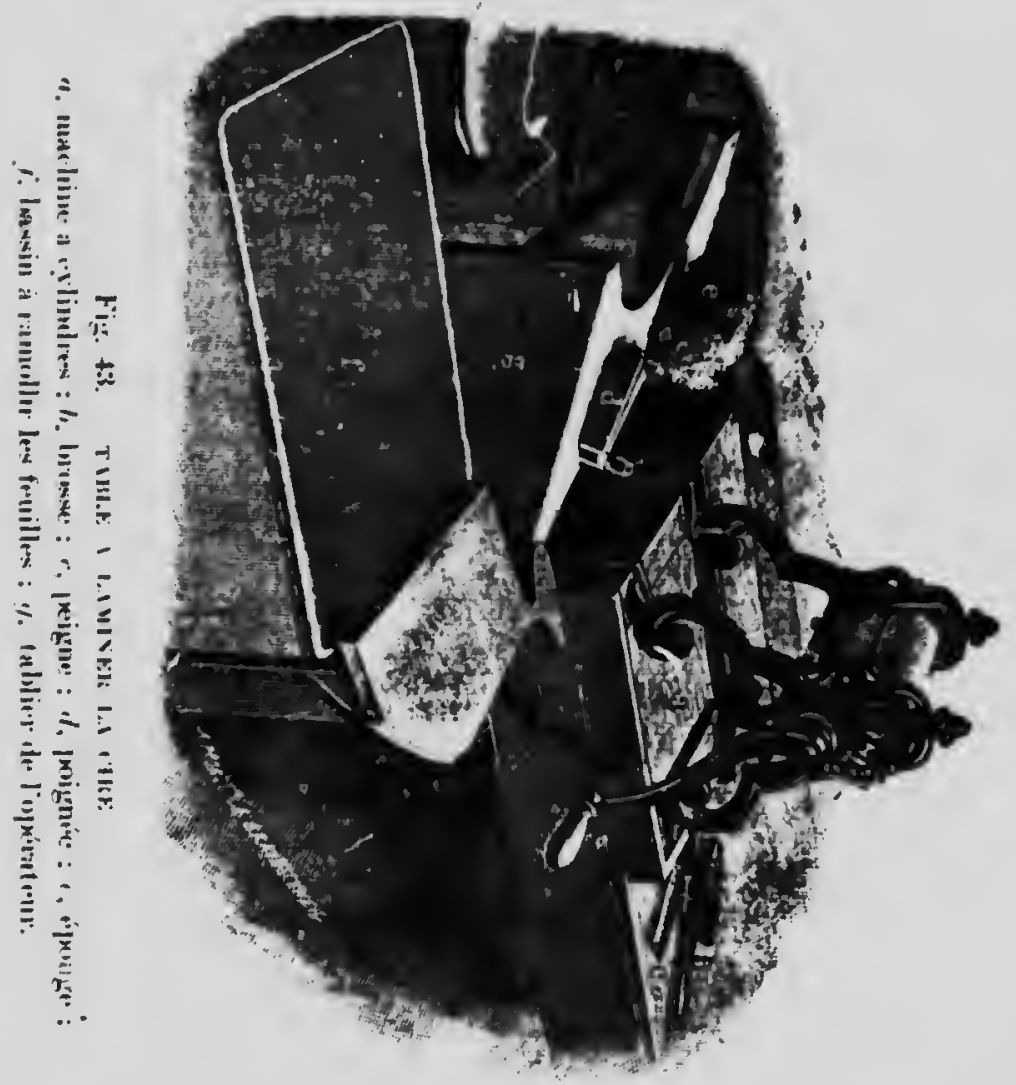

apiculteur venu peut tremper h cire an fonilles, la passer entre les rouleaux, comme tout cultivaleur punt fnire venir du tabac, rouler ses ıeuilles en cigares, mnis il e-t anssi diffcile pour une personne qui n'y est pas hahitué, le fuire une 


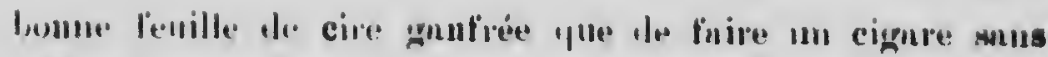
Iéfiut.

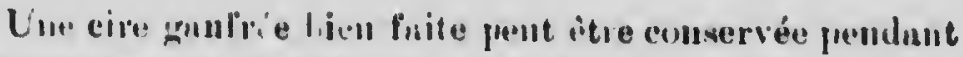

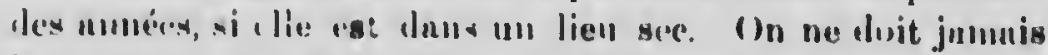

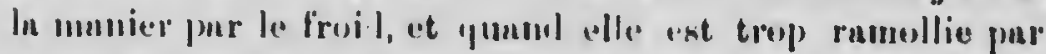

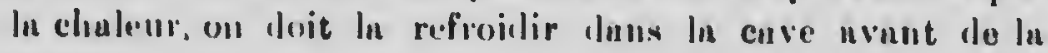
inunier.

Ia cire gaufrée est utfacliée 'nus les sections pur diffé. rents procédes. Daus son petit a, ige sur la conclnite les abeilles, M. Doslittle rlécrit ninsi sa ini' horle :

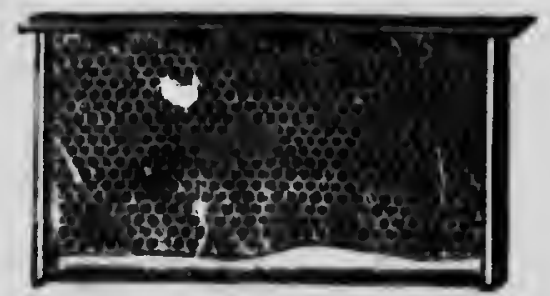

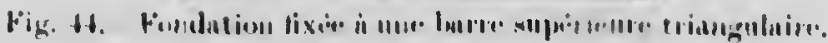

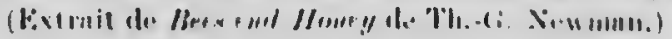

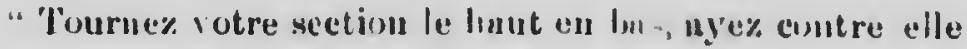
un fer chund et npres nvoir tenn ln eire presque tonchnit le fer retiru vite colui-ci et presse\% ln cire contre le looi-, nuquel elle restern co!lée"

l'our nitucler la foulation is une lanre supérieure triangulnire, "III pent ln presser s'e clingue citi comme dans la

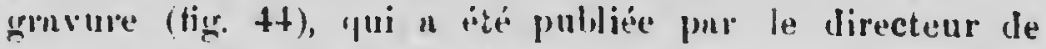
l'Americen Bre Jourmul. Mnis alle est leenucoup plus facile à uttucher qunud le lessous die la planchette est plut. On peut se servir pour celn le ln roulette insentie pur II. Hanlmugh, qui cultive les uleilles uvec suceies duns I'Illinois (tig. 45). Anjonrl hni l'on tait même une rninure lans la partio superieure lu culre pour faciliter l'opératiou.

On soutient en général la cire gunufrée uvec sles fils cle fer, mais ce s'est pus inlispensable. IJe til le fer empluye linl,i- 


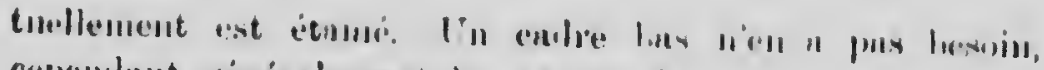

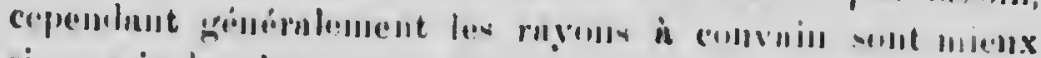

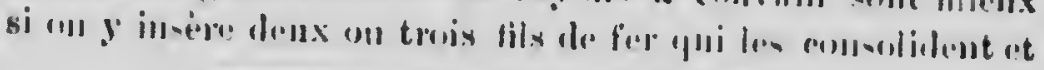

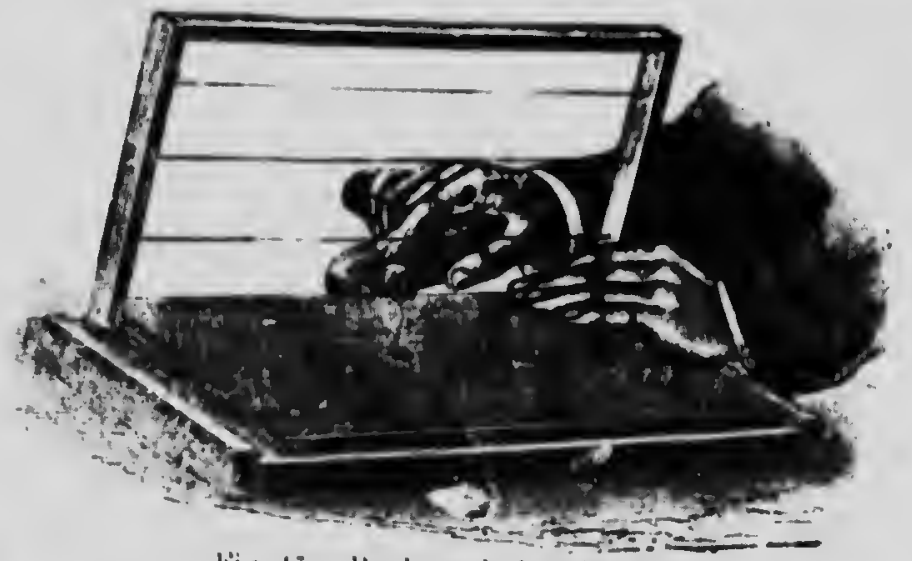

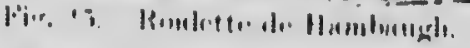

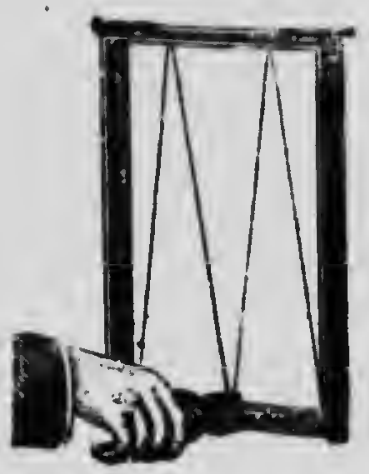

Fig. Hi. - Wunion ile ien-

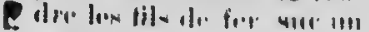
inilin.

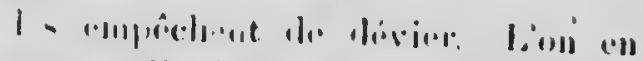

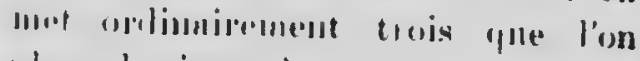

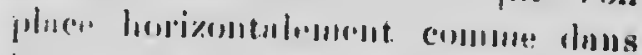

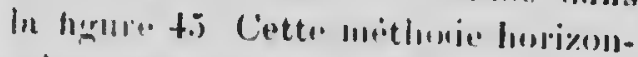

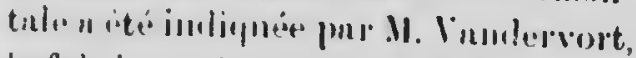

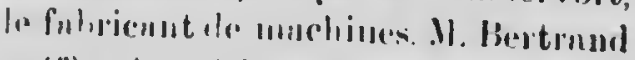

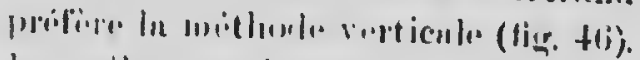

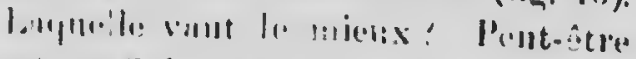

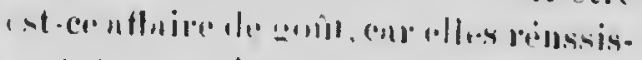

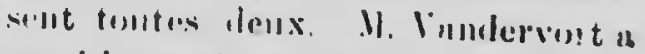

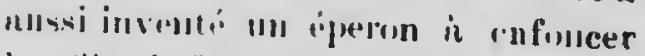
lese tils de fire dnus la cirr : c'ent une runlette de fép-libule inemtie sur

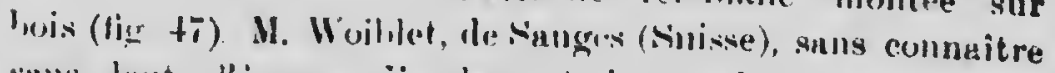
sms ionte l'éperon ianlervort, incentait le sien probu-

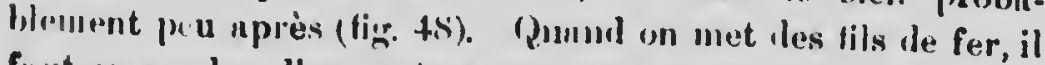
funt se comder d'en mettre ule trap erand nombre comme cer'tains apicultears l'ont fait nu délut.

Connme d'orlinnire, la cire ganfrée ent livrée en bandes il est pent-ritre necessnire de ionner des imdications sur la 


\section{$-11+-$}

manière de la conper en pieces ite in ganderer convenable pour les sections. C'n comteran que'conque sunit, pourvu qu'il soit bien aignisé. I'répare\% un patrun en hois dur de In grandeur exacte que vons désire\%; empile\% ave soin six on huit fenilles; place\% lessins le patron an l'nppuynut = uffi-

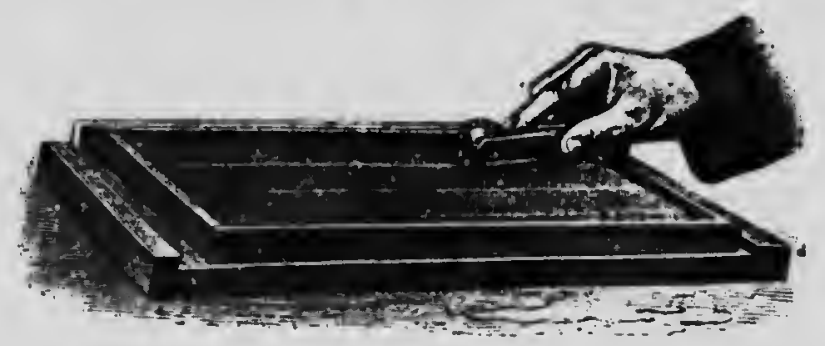

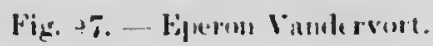

sanment pure le naintenir en pluce: plonger le lout de ia lame dh contenu duns de l'mul dre suson et si la cire est d la tempripatne convinalie, un - - ul comp de contenu tranchera les huit fonilles.

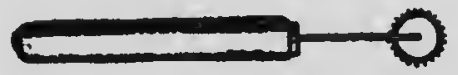

Fig. 4x. - Hikerun Wivill.1.

Y a-t-il un hon on mu maurais sems four suspe: dre lu cire ganfree dan-les conlres: Ou, '1n l'autres termes, deux

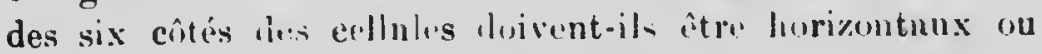
verticnnx : H!nber, ot Clieshire aprés lni, ont appulé l'attention sur ce fait que les aheilles comstruisent tomjomrs leurs collules avr c deux côtés perpendiculaires M. Cheshine expliqu tunt au long l'ajustement et les avantages le ce frit matnol et de son intuence sur la force du rayour. Il résulte le ses expli. cations que lu cire ganfréce, suspendue de cette fuçon. cest-é-lire avec reux"eûtés les cellules verticanx, sura

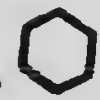
placée nuturellement, tandis que celle qui ser mise ainsi : $\Longrightarrow$ ne sera pas convennblement attuchée. 
Ia plupart des unachines qu'on emploie nujourd'hni font des feuilles qui loisent être placées horizontnientent pour que leur cellnles soient drus la position voulne.

On est agrénblement surpris de voir uvec quelle rapidité un essain comstruit ses rayons quand on lui a fourni de la cire gaufrée. L'enthonsiasme de l'apiculteur sous le rapport de son enploi est ellcore surpassé par celìi des alieilles lorsqu'elles lin trourent duns la ruche oì on les n logées. Cette invention mérite certainement d'être placée an même rang qu: celles des culres moliles et de l'extracteur de micl.

\section{DIAGRAMIE DES CADRES LES PJUS EN USAGE}
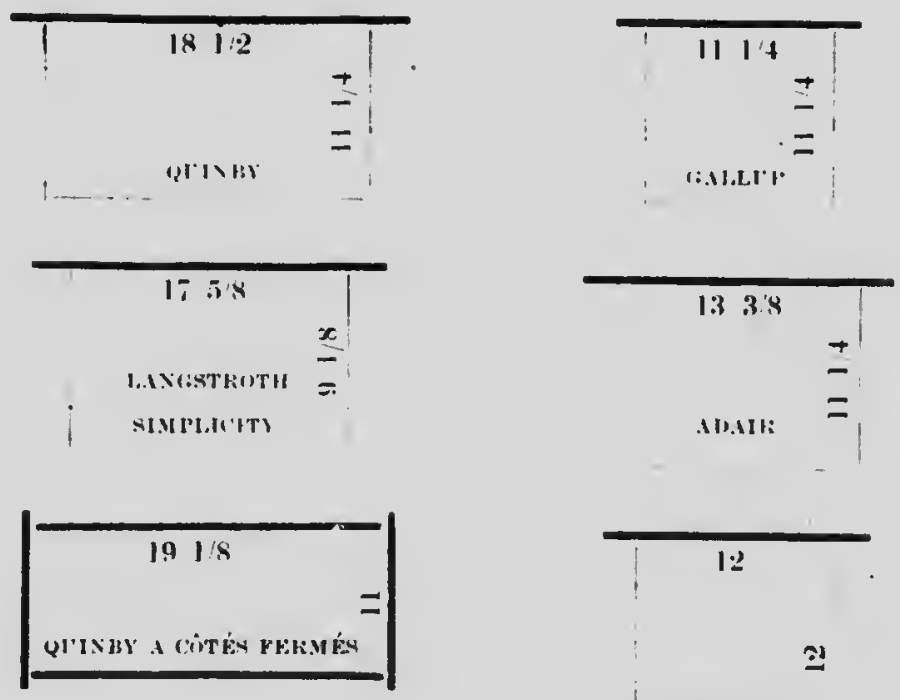

1:2

党

A บE์KITAIN

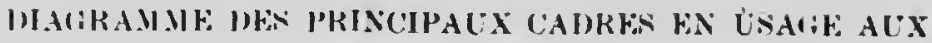
FTATS-UNIS.

Les chiffre:s imliquent en pouces anglais le:s dimensions extéritures. Los eadres suspendus ont des supports ile $3 / 4$ pouce, on leur traverse supirieure ent prolongéte d'autant. 


\section{CHAPITRE HCITIEIE}

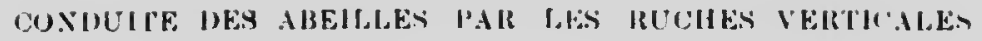

\section{Loes lo answers.}

Observations générales. - 'Tout ce que nous avons dit duns les chipitres précélents et ce que sern dit dams le chapitre snivant: sur l'étublissement du rncher, sur les opérations du printemps, les essuims et la mise en ruche, la visite d'automne s'npplique presque completement in la conduite des rncher repticales. II y a cependant qualynes différences dans la maniope d'opérer.

supposons que l'apiculteur possede nu cert in nombre de ruche's verticales peuplées l'aheilles: suivons ces colonies pendant tonte me sison, dep tis l:a fin de l'hivernugre jusidu'à l'hivernage suirunt, et imlinunns tontes les opérations quni diffèrent de celles décrites ponr les ruches horiznntales.

Fin de l'hivernage et visite du printemps. - En hivernage, les ruches verticales somt exhctement consti.nées cunıme des ruches hori\%ontales à loure culres, cur elles se composent simplement ilu eorjs ale ruche reconvert on nom du chapitenu. (Voir hivernement chapitre onzicme).

A près aroir supprimé les dispositions prises pour l hivernage, on fera la visite de toutes les ruches nu inoment vonlu (tiil avril) et on notera l'état de chnemue d'elles.

Pan chanue ruche, on anra soin de laisaer le convain dans les rayons du milien, on repurtera ì hroite et à gauclie, 
vers les extrémités, les rayons contenant le plus de miel, et te reste de la ruche sem garni par les ruyons construits en cellules l'ouvrières, vides ou contenant pen de miel; par cette disposition, ln mère ne sera pas grênéc lanss su ponte.

En faisant cette visite, on enlevera les rayous de niel aux colonies qui en ont le plus pour les donner à celles qui en ont trop peu.

Préparation des hausses. - On devra préparer d'avance les luasses garnies de culres, qui levront itre mises sur les ruches.

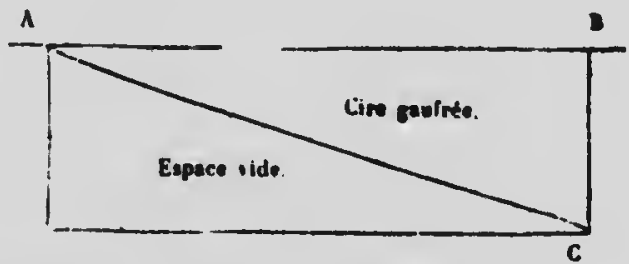

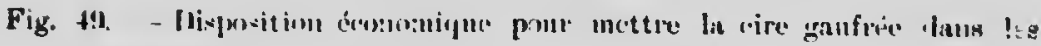
haussers.

Lorsquion a des ruches verticales depuis un certain nombru d'unnées, on aura un nombre suffisant de cadres pour garnir ces hansses.

Si l'on prépare des hansses pour des ruches nouvelles, les calres seront garnis de cire gauf rée, sauf un cadre vers le milieu qui sera entièrement construit, afin l'engager les abeilles ì monter dans les hausses.

Il ne faudrait pas se contenter de nuttre seulement des cadres aınorcés, car les hausses ne levant être placées que peu de jours avant la grande récolte, il faut que les abeille aient suffisamment de lâtisses disponibles pour profiter de tonte la miellée.

Cepenlant, on pent, par écomomie, mais si l'on ne peut faire autrement, employer la disposition suivante qui donne d'assez bons résultats. On inet, dans les carlres des 
hausses, des morcenux de cire gnufrée tniliès en triangle A, B, C, comne l'indique la figure 49. Le trimngle de cire gaufrée est tixée par deux de ses côtés $\mathrm{AB}$ et $\mathrm{BC}$, l. troisième $\mathrm{AC}$ formnnt la dingonale de la section on peut nussi placer l'amorce dans in traverse supérienre dn cailre.

Moment où l'on doit placer la première hausse.Le moment oi l'on doit mettre la première lunsse est nssez.

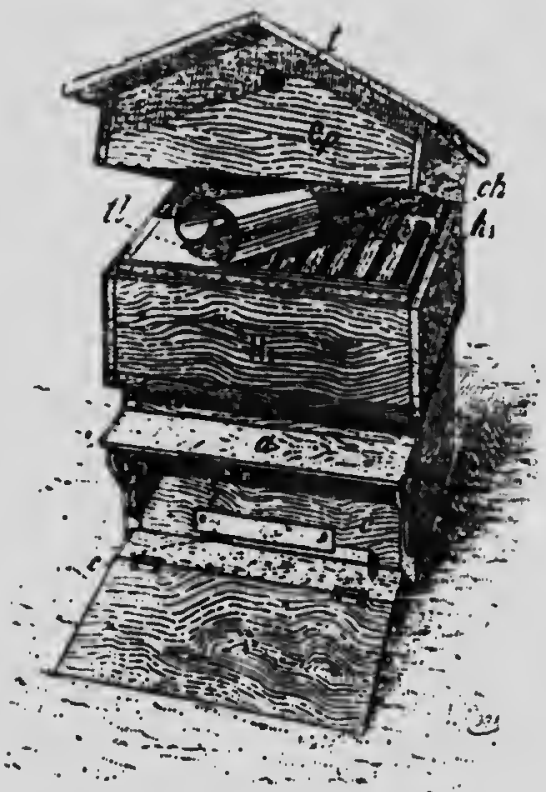

Fig. 50. - Kuche verticale, sme laquelle on a mis une premiere hausse ha; la toile "/ qui etait sur le corps de rurhe peromve la preniere hansse.

difficile à déterminer, et exige de la part de l'apiculteur une connaissance approfondie des ressources millifères de la contrée qu'il habite et de l'époque probable, où, d'après les circonstances atnosplıériques, il peut préroir l'upproche de la grande miellée.

En effet, le meilleur moment pour placer la première huusse est quelques jours cvant la grande niellée.

Muis une autre condition est encore nécessaire pour qu'il 


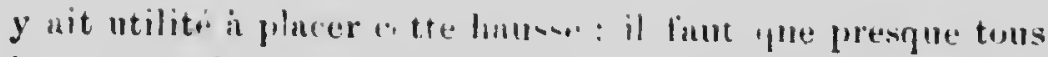

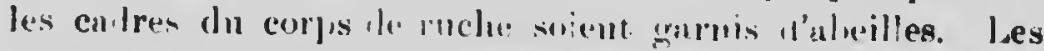
rnches trop finthles seront lone réunies dells it lens, si on veut qu'elles puissent profiter le ln grmmle miellér.

Duns le ens oir l'on ne ferait pas de réunion, il fanlrait attenlre le renforement des colonies faibles pour ponsuir y ajonter la premiere lanusse; mnis on risque alors bien sonvent l'uvoir mi. l... inmsses trop taml, c'est-d-dire uprès In grunde récolte.

Pose de la première hausse.-Pour placer la prenière haussi, on eu!ève le chnpitenn, on soulève la toile cirée par l'un des côtés, et l'on enfume l'une manière générale d mesure yu'on acliève l'o: sver toute la toile.

On :mlwite la haussp gnrmie de calres sur le corps de rucle: on remet In toile sur les culres le la linusse (t $t$, fig. 50), et un replace le clinpiteau $C p$ par-dessus.

Inconvénients d'avoir mis la première hausse trop tôt - Si l'on " placé les huusses trop tît, il pent arriver que par snite d'un refroidissement de tempérnture, comme la capaciti de la rucle a été lorusquenent nngmentée, les abeilles en se reswrunt soient forcées d'almulonner une partie du couvain.

Ce convin, non reconvert d'uleilles, peut périr et amener la maladie de la logne si rebutalle ponr le rucher.

Inconvénients d'avoir mis la première huusse trop tard. - si l'on n mis les hansies trop tard.

$1^{\circ}$ (II n'a pas protité de troute lin miellée.

2" La ruche linyant pas me enpacité sullisante, les abeilles pourront se disposer ì essuimer, et l'on sait quels gont tous lea inconvéniens 'pui résnitent le l'essainage naturel.

$3^{c}$ Il peut se fuire que la ponte le ln mère étant arrêtée par la miel lank la corps de ruche, la mère passe dans In 
bausse pour y eontinuer sn ponte ; dès lors, si on ailu couvain complètement éclos un près l'éclore, il est impossible de récolter utilement les hausses.

Surveillanoe des hausser.-Comme la miellée prit être plus ou moins nbondante et lurer plus ou moins longtemps, les hausse's se remplissent plus ou moins vite suivant les circonstances, il est lonc nécessuire de strveiller les hausses de toutes les ruches, atio de closisir le meillenr moment pour njouter les secomrles hausses

Certuins apicultears facilitent cette visite ì l'airle de hausses vitrées l'un côté, lu vitre étant recouverte par un volet qui peut se tixer par un crochet.

Pose de la seconde hausse. - Si ln miellée est suffisante, les premières hausses se rempliront de miel, mnis il ne faut pas attendre qu'elles soient pleines, car on sait que les abeilles ont toujours besoin d'une assez grande surfuce de bâtisses pour f́nire évaporer l'excès d'eau nvant d'operculer le miel.

Le meillenr woment pour chaque ruche est d'ajoucer In seconle hansse iorsgue la première est aux deux liers pleine. Mnis cette hausse ( $h_{2}$, tig. 51 ), on ne l'ajoute pas par-ilessus la première, on l'intercale entre le corps de ruche et In première linusse, $\dot{x}$ la place qu'cccupnit cette première hunsse. Cette lernière, h1, est mise pur-llessus ln secomle hausse la : et conserve sn toile cirée $l l$ (tig. 51).

Cette maniere d'opérer offre l'arnntage de haiser des rayous vides non loin du corps de ruche ce qui engnge les aheilles ì les remplir plus nctivement.

A vant l'ajouter une seconde hansse, il est prudent ile visiter ia prémière, pour voir si elle ne renferme pas de couvain. Cur, dans ce cas, il fuulrait mettre $\therefore$ seconile hausse par-dessus la première; si on la mettait par-desonus, Ia wite pondrnit duus cette secoule liansse encore plus que 


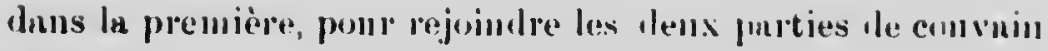
sépurécs.

Nous avous dit phus linut que les meles verticales varient quani mo nombre de lenrs culles. Je plus grand nombre l'npicultenss se servent de mches contenunt ile 7,8 ou 9 cadres, tant dans la chambre de comsinin que dans les hansses. Nons devons cependunt mentionner le cas de M.

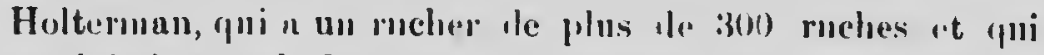
enploie la grunde Langratroth a donze conlese. Ansi somrent. n'n-t-il besoin que al'me hausse par mehe.

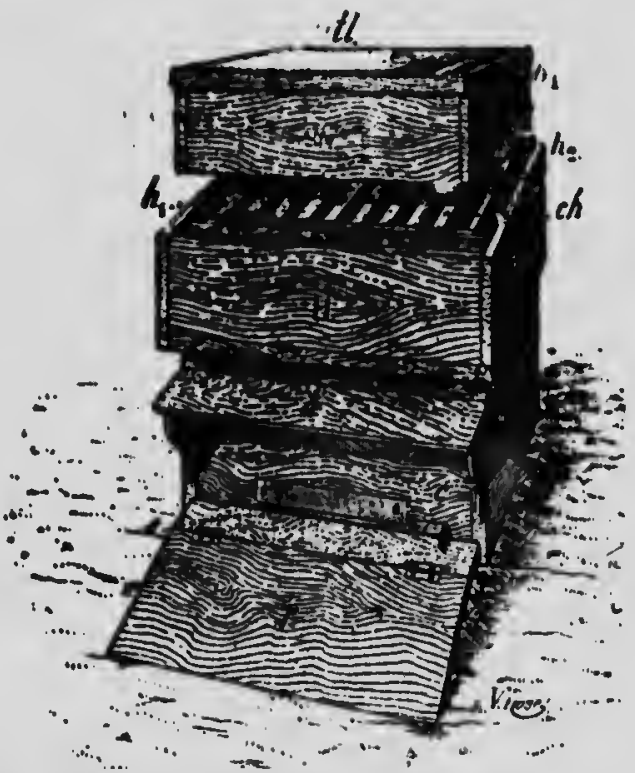

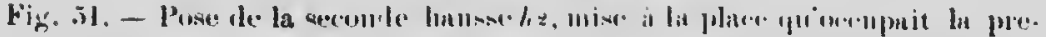

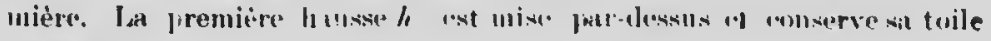
cirie 11.

Pose des hausses pour la miellée d'automne.-1)ans les contrées oì la récolte prificipa!e a lieu í !n tin de la saison, c'ent naturellement à lin tin cle l'été c'est quelques jours avunt ln granile micllée probuble (nue l'un doit placer les hinsses. 
Dans les contrées ai il y 1 ume miellée le printimps et unc miellée linutomne on levrn puser les linases nu prin. temps, los réculter, les re lonner pinl In miellée l'nutonne et les récolter le murenn ; ees nombrens.'s opérntions ont l'avantuge de permettre de miens sépurer les miels des diverses éporgues. Chez nous ln grmule miellie est grénérnlement terminé uा 1.5 munt.

Visite des ruches lorsqu'elles ont des hausses - Sii,

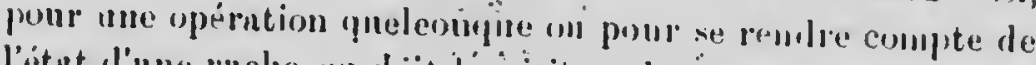
l'étut l'une ruelre, an doit li i isiter nlors ju'elle posedte une ou plusieurs lmusses, on opreve fe lin maniere suivante: on retire les linusses, on les place sur le sol et, avant le visiter le corps de ruche, on enfune trè fortement.

si l'on étuit obligéd de fuire cette opération à une époque vit il "'y ait pus ale miel dans les Heurs, il fundruit se ilétier dil pillage.

Réoolte des hausses. - Il ne fult pas trop se presser de récolter les hausses, car le miel qui reste ussc\% longtemps lans les lıusses est operculé en plus érumlu quuntité, et pur conséquent de meillenre conservition. Pinle récolter nue hausse, on opère de la fay in yui suit :

On enlève le chnpitenu et, nu moyen l'ure nhondnnte fumé ; on force une purtie des abeilles de redesce: dre vers le bus 1: on visite nlors rupilement la linuse ponr roir si elle ne contient pus le comvin, car, lan; ce ens, il faulrait Inisser In linusse sur In ruehe juspu'a l'éclosion complète du couvinin.

S'il n'y " pus de convain, ce qui est le cons le plus ordinaire, on détache lu hnusse par le lons nu moyen d'un ciscan;

(1) Pour astiver la descente des alseilles ver's le corps de rnchie, on pent se servir cie ne qu'on appelle la tolle phéniqu'e. On verse dans un sean d'ean la valeur d'un petịt verre a vin d'acide phéni,gue. On trempe la toile dans cotte solution et, après l'avoir tenilue on la place sull la hansar. L'mleur de l'acite fris tronendre eu partic lea aleilles. 


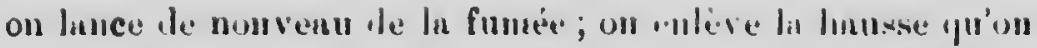
phace provisoirement sur un tahouret; "1n remet ln tuile snr la hausse de dessous oul sur lo corpas de ruehe, et on replace promptencut lo clnpitenn.

On emporte successivement les linusses dins lo laboratuire; on les dispose elineune sur um! cale et on recourre te linut nvec les twiles.

Les nbeilles qui sont restées ibns les hnu-ses, comprensut qu'elles sont isolées le leur ruehe, sortent pen ì peu par le dessons des humsses et s'envolent vers les fenêtres qu'on ouvrita le tempi en temp; pour les laisser rejoindre leurs colonies '

Les myons de miel, retirés less lubusses, sont rusnite passés a l'extracteur.

Puis on remet les cadres vides dans les linusses, et le soir on replace les hansses sur les ruches pour fnire nettoyer ces cadres par les abeilles. Ponr évitor le piliagra on rétrécira les portes $d$ : tontes les rnches peminnt quelques juars.

Visite après la récolte. - Il va saus dire que l'on doit visiter toutes les ruches uprès la récolte, ou exuminera dans cette visite si chaque corpi le ruche contient environ 30 à 32 livres de miel pour la provision thlyiver. (Un rayon de uniel de 144 pouces carrés, pèse généralement un peu plins le 8 livres.)

Avec les ruches verticules, commot ln plus granle purtie dn uniel récolté se trouve luns les hunsses, il peut urriver que le corps de ruclie contienne trop peu de miel.

Il fuudrait donc, à l'automne, ponvoir prendre des rayons de miel duns des hausses, pour les plucer dans le corps de la ruche. Mais cela ne put se faire facilement que si les cadres

(1) On a prétunise! pour la récolte des hansses un appareil spécial appelé chanse-abeilles; nous en parlerons an chapitre ot nous traiterons le la recolto du miel, mais ou up're plus rapilement par in methote que nous venone de ilecrire. 

des liansses sont de la même grouleur que cenx du corpos do
ruche.

Un upiculteur exerci lont les condes bes linusses ne sont pus de même dimension fue ceux du convnin ponrrn souvent éviter le nourrissement l'uutomme pui offre beauconp d'inconvennents, s'il snit enlever les linusses ou moment

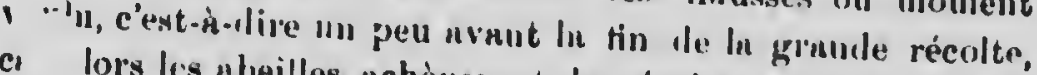
cr lors les abeilles nchèveront In ricolte en trunsportunt lc. . I lirectement duns le corps le ruclio.

Mise en hivernage. - Nons nvons dit qu'en hiver, les ruches verticnles ne Iri, 'nt pns porter de liausses.

On pourrait croire que si ln provision de miel u'est pas suffi. sunte duns le corps de ruche, il suffimit le luissor nne hausso pleine de micl pour assurer cette provisic Mais, si l'hiver a des périorles de froid prolougé pondant lesquelles les ubeilles ne peuvent plus se déplacer, cette hunsse pleine do miel ne servira it rien $n \| x$ abeil!es.

En effet, le groupe des abeilles installé dnns le corps de ruche ne pourra pas, dinns ln snison froide, passer du corps de ruche dans la hunsse à cause de l'intervalle qui sépare inévitallement les ruyons de ces leux purties de l $\Omega$ ruche.

Qunnt in ln mise en hivernnge du corps do rnclie recouvert ou non de son chapitruu, nous en traterons dans lo chapitre onzième.

\section{MIEL EN SECTIONS.}

Sections. - On peut se proposer simplemest le fuiro f our soi quelques sections ntill le consonumer le iniel is tuble sous cette forme élégante; alors un ties grand nombre i'o sections ne sont pas nécessnires.

Si, au contraire, le cultivateur dibeilles veut fuire des sections en grand pour les venilre, et s'il est sûr d'en tronver 


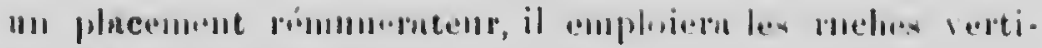

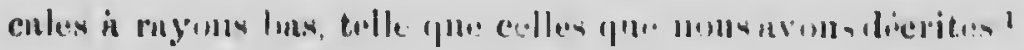

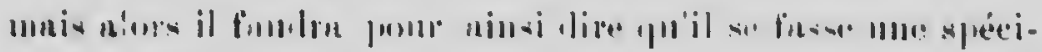

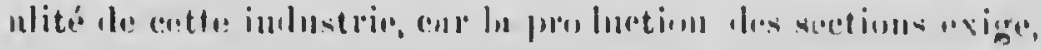

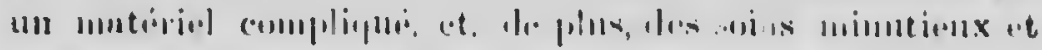

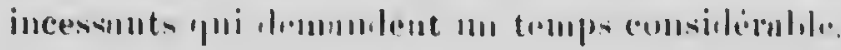

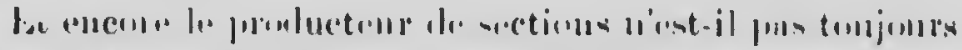

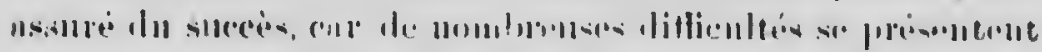

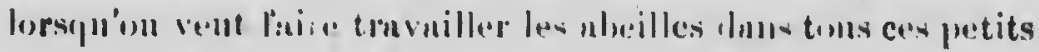

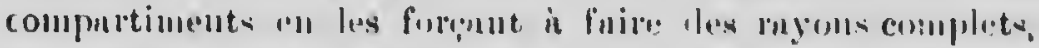

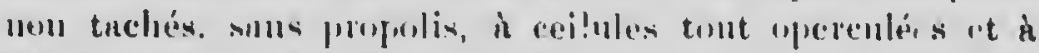

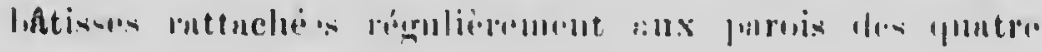
côtés.

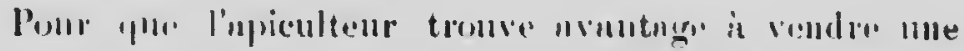

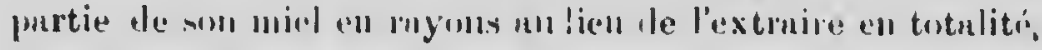

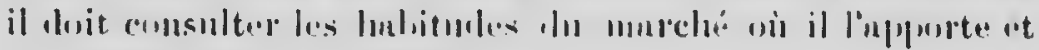

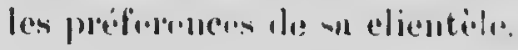

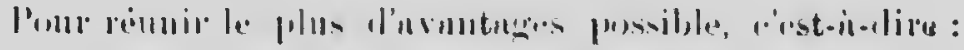

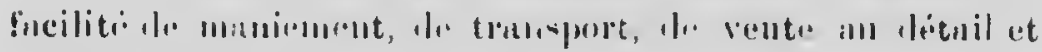

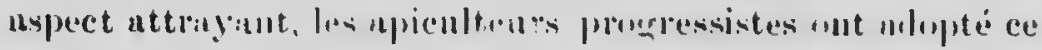

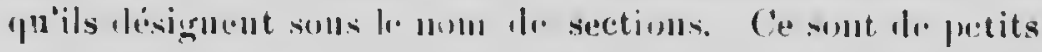

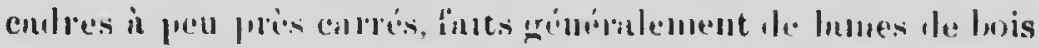

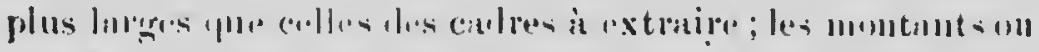

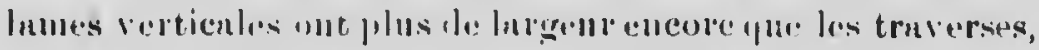

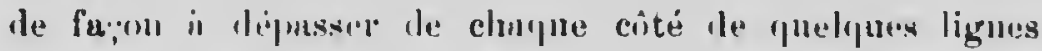

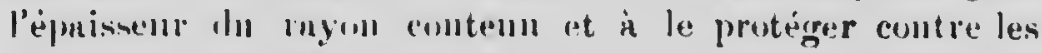
chucs (fig 5:2 et 533). I sex dimensions des sections sont culcules de munière it ce que, pleines, alles se rmprochent le

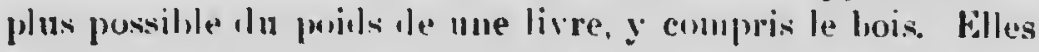

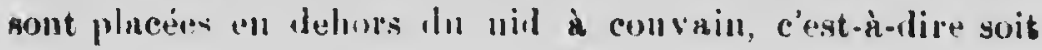

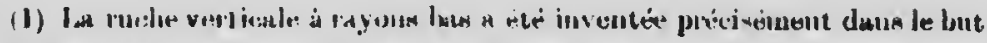

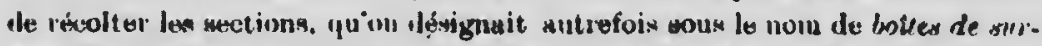

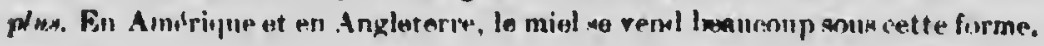




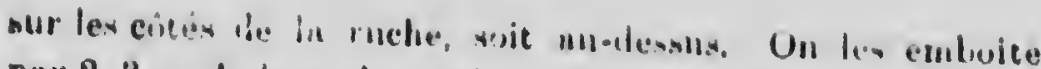

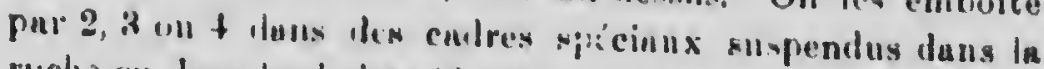

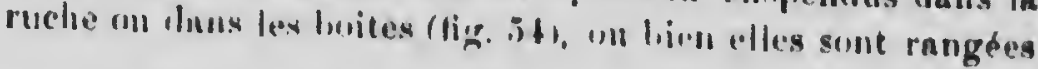

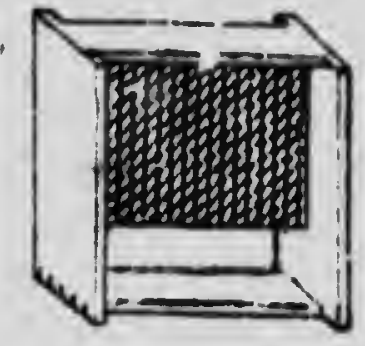

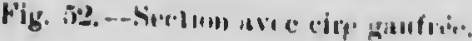

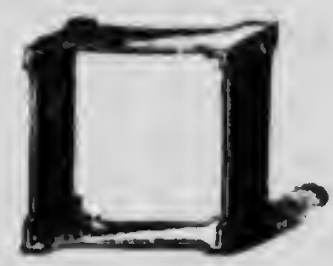

Fig. i3. - Freclion asapmblise.

sur des chisvis à claire-voir ; mnis dun. ce chs elles doivent nécessairenent itro plucées m-rlessus hu nici à couvain (fig. 54 bis, in, .ji)

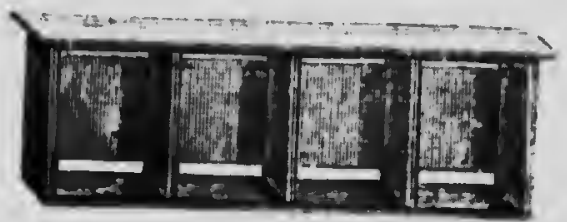

Fige in. - Condre à sectimns

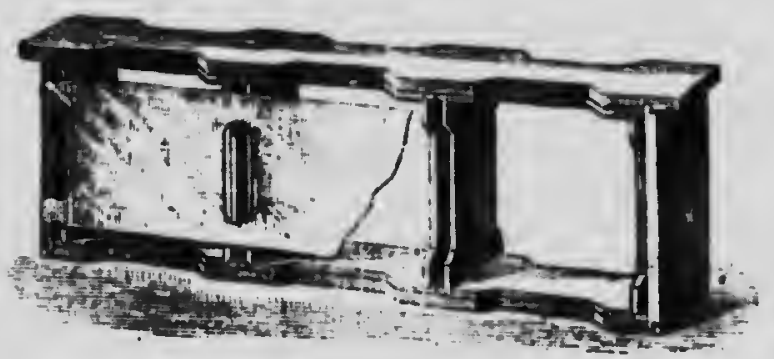

Fig. 54his. - Ciulre pour sections a yutre passages.

Comme nous l'avons déjà dit ces petites sections de rayons doivent être aussi propres et coquettes que possible, 


\section{$-\cdot 1: i-$}

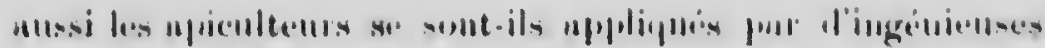

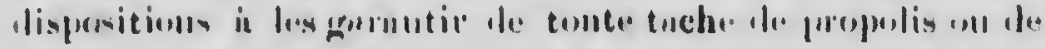

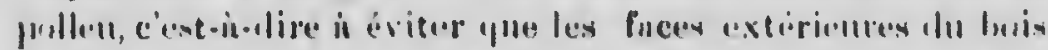

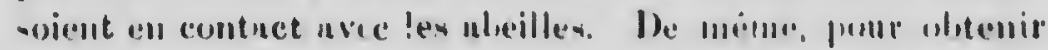

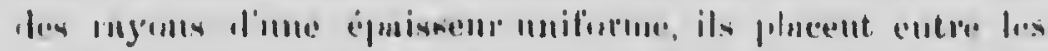

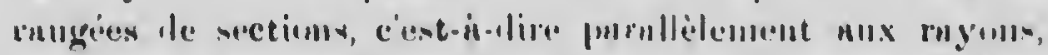

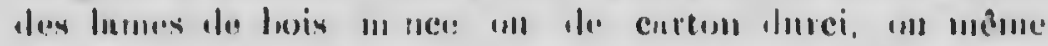

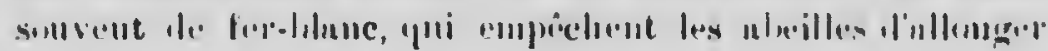

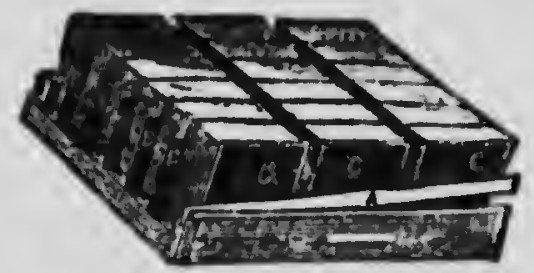

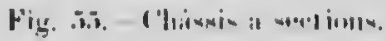

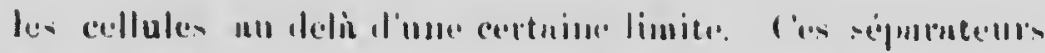

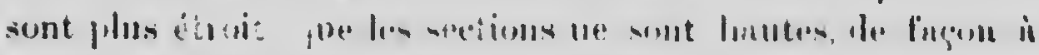

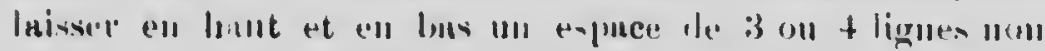
fermé Ils sont clones l'un cirti uns culles contennut les

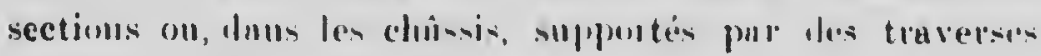
clumeres an find di: cerox-ci.

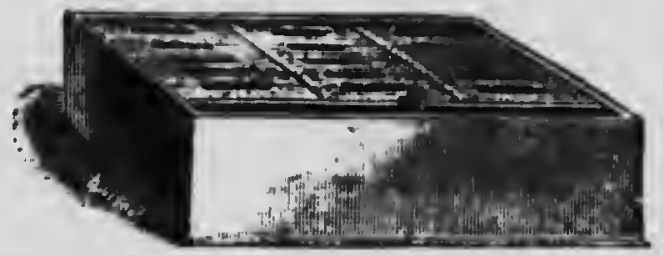

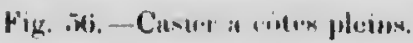

Les sections I s'uchìtent 1101 montées ; l'nsisemlinge se

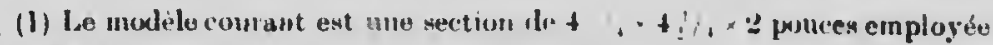

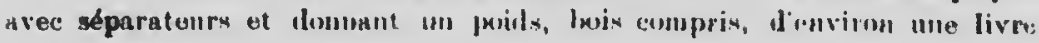
anglaise. 
fait in mortnicas et temons, on hien ln section est finite l'mue senle piece yum |r. " mux pluces ait se tromvent des cumblu-

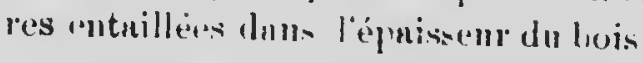

Ccmment on prépare les section: pour les abeilles.

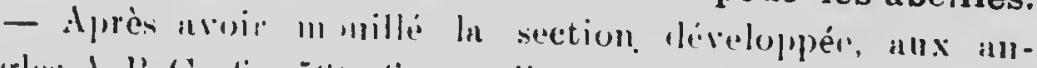

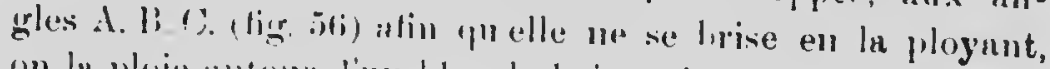

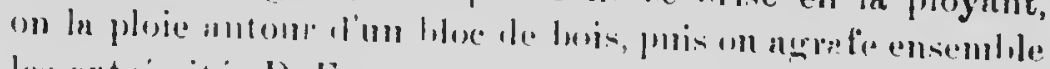
les (extrentites J) F.

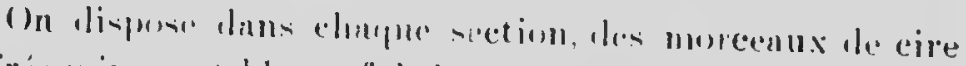

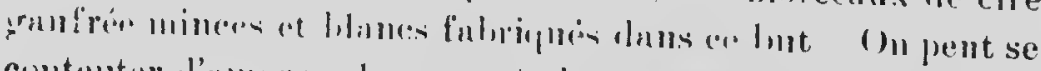

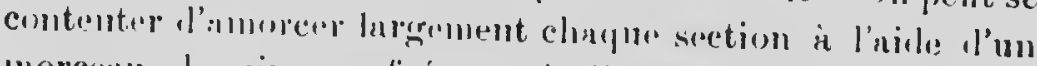
morcesu de cire contire; mais il est préfernble, pour ln

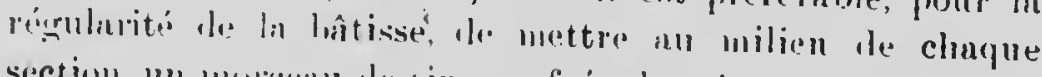

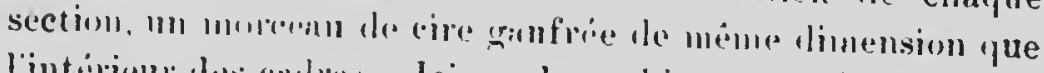

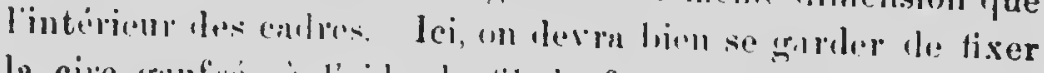

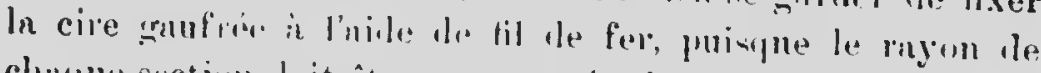

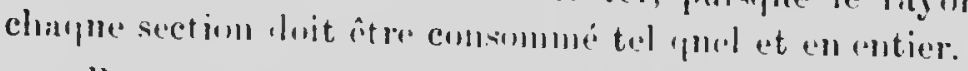

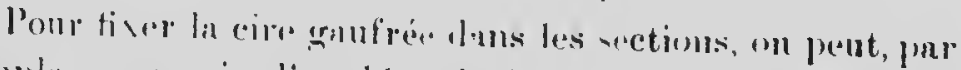

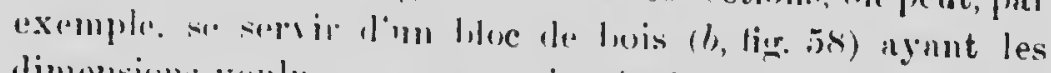
dimmsioms vomlux, mo pen moins andis que la moitié de ln saction; an enculla l. bluc avec la section, on pusa la cire

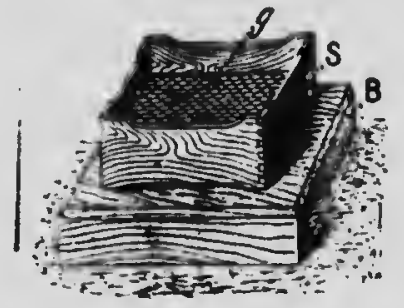

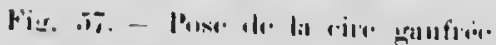

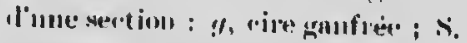

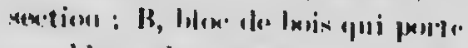
III hlox plus getit ll. tig. ix,

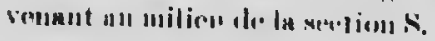

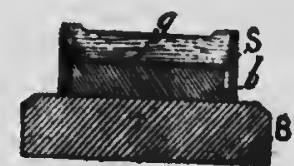

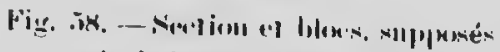

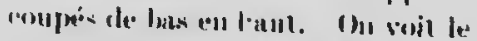

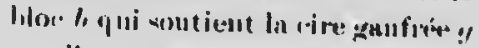

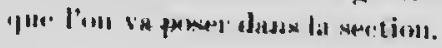

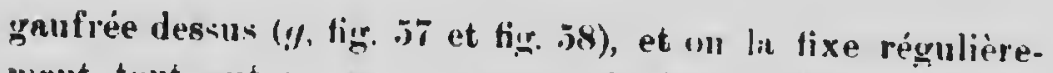
nent tont nutour en $y$ versant de la cirs fondue Pour 
verser cette cire, qu'on a fait fomlde dans more hurette is hainmarie, on incline it la foris la section at lo bloce coll soutelnant

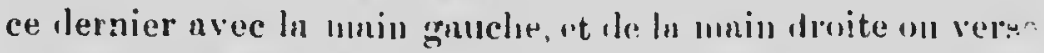

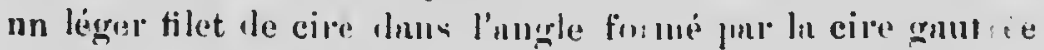

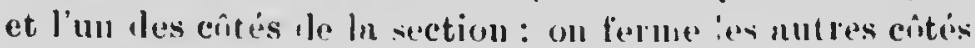

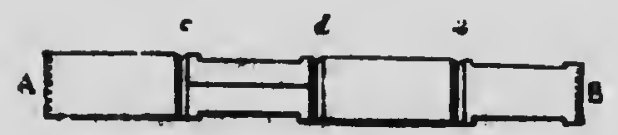

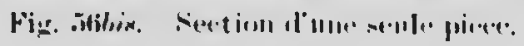

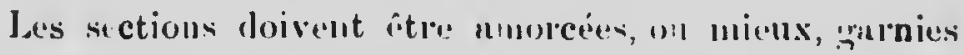
de cire ganfrée tres mince. Arre le petit instrunent Parker (fig. 59), la besogne se fait beaucoup plus promptement. . Cet

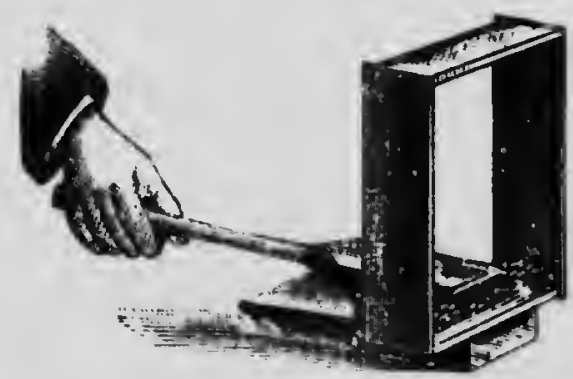

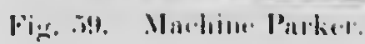

instrument se compose l'un levicr relie in une phanchette qui se visse sur me tahle. Apres noir en luit le levior de miel; on place la section sur la plancliette contre l'arret et dessus on introuluit ln fenille ơнufée jusqu"n ce qu’elle dépasse un peu la moiti: de la largeur le ln section; on releve l'extrémité du levier en serrunt et on plier la fenille à angle droit contre celui-ci, qui est ensuite rotiré. Le loorl de la fenille se trouve pressé contre la rection. Jes dimensions .le l'instrumeut loivent être uluptées à celles le !a section. Les feuilles étant sujettes ì s'nllonger, $\vee u$ leur extrêne uninceur, il est bon de laissel un espnce vidle sur les.côtés et suriont en bis, comme pour les cndres. 
(1) intombit dinillems chupue joms des perfectionne. ments dun la fulvication des sections et la pose des feuilles.

locs rections garnies ninsi de ciro ganfrée, sont placées dams les casiers avec les sépuratenrs, et on les serre les unes contre les antres pour empécher le plus possible la propolisintion sur les borls.

On phec les consicis ainsi gurnis de sections (tig. 50) an-dessus du corps de ruclie, et après avoir recouvert l'ensemble nvec la toila, on reme te clmpitenu. Les casiers a sections luivent être placés comme les huusses, c'est-d̀-dire quclunes jours nvant ln grande miellée.

A pritir le ce moment il faud'n surveiller les cosicrs de la manicre suivante:

l" Coumur les aleilles commencent par remplir les sectims /n milien arant d'avoir terminé celles qui se trourent sul les cotés, il fuulrn retirer celles du mulien dès quidles seront completement aclerves, les remplacer par collis denceités qui : a sont pas achovón, et remplacer ces deribien prar des sections vides:

2" Il rest importint de retirer les sections au moment oi elles viemment l'être terminées, car ill séjour trop prolongi der aludlles sur les sections opercules nurnit l'incon. vénient de les tacher ;

3" Les sections retirée qui auraient par trop le propolis scront grattées avec le plus grand soin, opérntion délicatu qui exige une certaine adresse de main.

Inconvénients à éviter lorsqu'on fait du miel en sections. - Lie promière lifficulté consiste dans ce fuit, que les uleilles sc lícilent parfois difficiloment à monter duns les sections; "m les engngera d̀ monter en remplaçant quelques sections du milicn par des sections préparées, non avec de la cire ganfrée mais avec les rayous tout construits.

On a bien cherché à hâter la montée des ubeilles dans les scctiuns en réluisant le nombre des carires dans le corps de la ruche, par exemple, en ne laissant au milieu que 5 à 
6 cadres qu'on isole du corpis le ruclet, à droite et à gauche, au moyen de planches le partition. Mnis III tel procédé présente deux inconvénients: le pre'nier, c'est que les ubeilles ne conaruisent pas volontiers lans les sections qui sont placées à droite et ì gonuclıe au-dessins less espaces laissés vides dans le curps le la ruche; le second inconvénient, qui est le plus gruve, c'est que, tout en réussissant pour les sections, on court risque, par le trop grand rétrécissement du corps de ruche, de ne plus laisser la plnce suffisante pour le développement du couvain et pour les provisions d'hiver, ce qui peut compromettre, dans l'arenir, l'existence de cette colonie.

Une autre difficulté, cint qune les ruches sur lesyuelles on dispose res sections, mit grénémlement une tendance plus grande à essaimer, ce qui, unus le sivons, offre les plus grmols inconvénients. Il ny a pus de remèle pratique contr" cette difficulté.

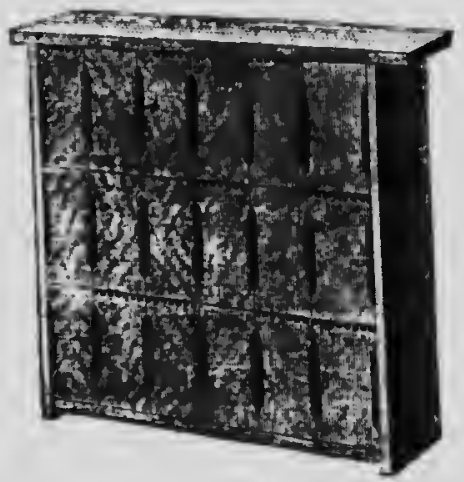

Hig. "

Enfin, si le châssis à suction u'a pas été placé en temps voulu, il peut se fuire que ln mère monte an-dessus du corps de ruches et vieme pondre dums les sections, ce qui les perd counplètement, unis ce cus est très rure.

Miel en seotions avec les ruches horizontales. - On" peut nussi faire des section- nvec les ruches horizontules par l'un des procédés s:rivants: 
10 Ayaut préparé les sections commo il est lit plus haut, on dispose le casicr qui les contient (fir. b0) sous le toit de la ruche horizontale en le posnut sur le dessus des cadres ou se trouve le convniu. On a ell soin de retirer d'avance les lattes qui sont entre les cndres on les planchettes qui les reconvent.

La ruche horizontale que nons avons decrite est combinée de fuçon ì permettre l'intradnction suns le toit, l'un tel casier in sections. Loropln'on met les sections sul les ruches hori\%ontules, il funt avoir soin de les placer sur les ruches les plus fortes.

$2^{\circ}$ On pent anssi disposer les sections verticulement, placécs lans un calre nuśnngé luns ce but (tig. 60): muis ce

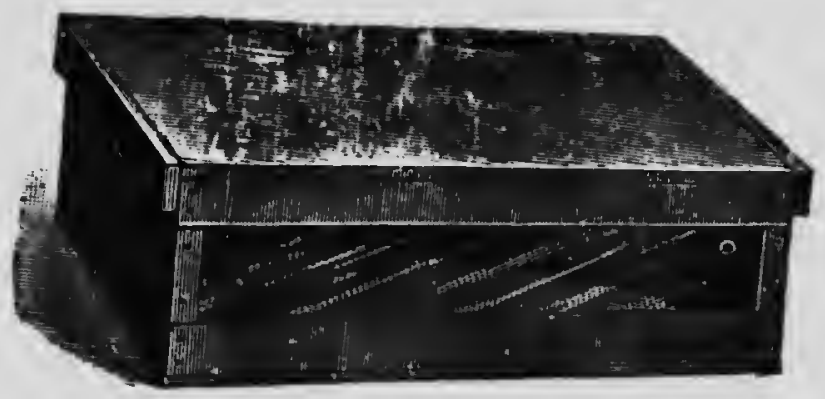

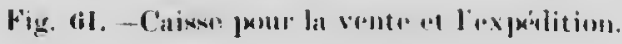

cadre ì sections, plncé ninsi, présente l'inconvénient que les abcilles travillent plus vers le lant. On ie plnce in ln suite du dernier rayon de couvnin

\section{E.PPOITION DES SECTIONS}

I wes rections s'om'mllent at s'expéslient par 3, 1i, 12, etc., Jans de patites oniswes de mesire ex rcte que les fonrnisseurs livrent non asscmblées (figr. 61!. Well des côtés des caisses

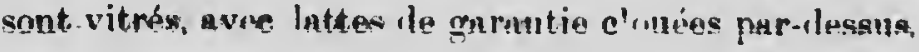




\section{CHAPITRE NEIVIEII:

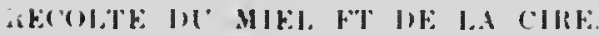

\section{Minl Eetruit}

Pour séparer le mie! de la cire, les apiculteurs de l'mucien régime fondai nt on hounaient les mọnus et les fuisnient égoutter.

Les apicultenrs soignenx séphrnient leurs miels en différentes qualitis. Les ruyous neufs, le couleur claire, dommient nu uniel légrer en couleur et pur ; les myons qui avaient contenu dı comvinu, un miel trouble et de qunlité inférioure.

Ces méthodes puimitives furent ensuite grumetent amóliorées, comme un (in̂tinnis, pur exemple, vù les npiculteurs fuisaient fondre les rayons an soleil ponr séparer le miel de la circe fommlue.

Cess conditions n'ont pus "xisté en Anérique du moins

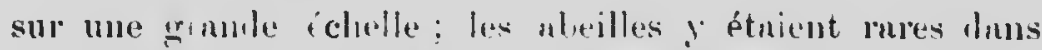
le prineipe. Les colons mérienins avatent trop it faire pour s'secuper d'ubeilles: le petit nomlire de cenx qui en possédaient etonftuient de temps en temps une de leurs colonies et consomminint le miel mil logis; cenx qui en possélaient un plus grand nombre venluient purfois quelques rayons brisés à leurs roisins et que'ques livres de miel coulé au pharmacien, qui étnit peu llifficile, étant liabitué all inie! de Guba épais et trouble. Petit d petit, cependant, les conditions favorables offertes $\dot{x} u \dot{x}$ abeilles par les terrains incultes tirent remplir d'essaims les 
arbres creux les forets vielges et lo chasseur d'ulueilles nAquit. Des milliers d'albres tombèrent sous sa hache pour lui liver le miel qu'ils contemient. Cr.ttc apicultur grossière et l'ocension, si un pent nomuer cela de l'apiculture, produicit compurativement le grumles quantités de miel : mais comme ce miel provennut tonjours de rnyons brisés, étnit mélangé dle pollen, l'uleilles mortes, de larves écrasées et le bois pourri, nu prit l'lubitnde de le faire bouillir pour nmener à sa suf face la cire et les impuretés qu'il contenait et qu'on écumnit. Le résultnt ol,tenn étnit un liqunide mulpropre et tronble, foncé ell conlenr et fort cu savcur. A côté de ce liquide peu uppétissnut, on venduit queligues bemnx rayons de mill, qui créèrent unc préférence pour le miel en rayon.

Cependant, quand ils consiciéruient ce que les rayons cuntaient nux abrilles an miel, en temps et en travail, les apicultenrs les plus arnncés souhniśtient, surtont depuis l'invention du rayon molile, de trouver le moyen de sortir le inicl des ruyons sins les détériorer, utin de pouvoir les rendre nux abeilles, nuxquelles ils serviraient incléfiniment.

En 1s65, le inajor de Hruschka, je Dolo près Venise, inventa le smelutore, ou mélextructeur. Voici comment il fit cette invention; il avait donné h̀ son fils un morcenu de niel en rayou sur une assiette. L'enfunt mit linsiette luns son panier et fit tourner celui-ei autour de lui comme si c'eut été une fromle. Hrushka remarqua que le miel avait été solti ln rayon par le mouvement et en conchut qu'on pourrait vider les rayons par la force centrifuge.

Cette invention fut acclamée par les apiculteurs avancés de tous les pays, coinıne égale ì celle des rayons uobiles et comme le complément de celle-ci; et ellc méritait bien cet honneur.

En présence des faits précédents et après une expérience de trente ans du mélextructeur, dit Dadant, nous engageons fortement les commengants à produire du miel extrait s'ils peuvent en tirer la moitié du prix qu'ils obtiendraient du 
miel "ul rnyon. Nous uvous détuillé les aviutages que les abeilles retirent de cette production, quand on leur forme des rnyous, voyons maintenant ceux qu'en obtient l'npiculteur.

10 Il peut surveiller et signer un plus grand nombre de colonies. Les manipulation d'un rucher conluit pour produire du miel extruit demandent moins de la uoitió du temps requis pour !a production da miel en rayon. Nos plus grands producteurs de mitel en rajon reconuaissent qu'une seule pcisonue ue peut soigner avec succès plus de deux cents colonies quand elles produisent du miel en section, tandis qu'un seul npiculteur peut en soigner plus de cinq cents, placées en différents ruchers, s'il fuit du miel extrait.

Il va sans dire que pendunt les jours où on extrait il fuut se fuire ailler; mais on n'a pas besoin le gens versés dans le métier, qui seraient difficiles à trouver. Le coût de leur travial est remboursé par les opercules, qui rendent plus de cleux livres le cire de toute première qualité pour chaque deux cents livres de iniel extrait. On ne trouve pns une semblalile compensation dans lia prorluction du miel en section.

2" Qumul ou proluit du micl extrait, les myons de surplus sont conservés pour être dommés uux ubeilles lors ile la récolte suivante. Cette mẹtloule enjếche virtuellement l'essuimage maturei te permet in l'apicultenr le gouvermer l'uugmentation du nombre le ses culonies suivint ses lésirs. Un des npiculteurs pui obtiennent le plus ale uniel en rnyon aux Etats-Unis, M. Manun, qui " venlu quin\%e tonnes de miel en section en 1855, nuus a avoue qu'uvere su mithorle de production de miel en section il ant presque inpossible de contrôler l'essaimnge naturel et que le moment vi il aurait trop d'abeilles n'étnit pas éloigné. II posséduit alor's sept cents colonies. (Dudant).

Le cultivateur, ou l'amateur, qui tient seulement quelques colonies afin de proluire du iniel prur su tuble, trouvera 
'u'il est hien préfirnlile re faire lu miel extruit. Ave trois colonies dinbeill's (t) 111 extructeur, Inus un pays ile production ordinaire, on peut compter sur 150 a 200 livres lo miel en moyenne chaque annér.

Pour vhtenir lu plus gmmic quantité possible de miel extruit, lu colomin ne loit jumu is monquer ,le reyons vides.

Aussitôt que les rujouns l'uue des boîtes sont à peu piès aux trois quarts pleins, l'on met une nutre boite sous in pre nière ot quelquefois me troisiène sous cette scconde, snns attendie que le miel soit cncheté; mais l'ou ne sort jamais les boítu's, pour 'xtraire le miel, nvant que la récolte soit terminée, car il ent pu'érable que le miel soit alsolumnat inûr.

Le miel est mûri un évaporé par les courants l'air que les abuilles produisent par leurs battements l'ailes et par la grande chaleur qui rénulte de cet exercice A uesure que le miel est évnporé il liuninue de volume, et tout celui qu'elles npportent jouruellement, qui est plus ou moins aqueux, est placé dıni des cellules en partie remplies de iniel déjà érnporé. C'est pour cette mison que le miel qui n'est pus encore operculé, qunud la récolte rst terminée, est nussi mûr et quelquefois plus uû que celui qui n été operculé durant la récolte. Fu ceffet, yuand le nectur ist abondant les abeilles ferment sonvent les cellules trop tôt et le miel yui y est enferué pent feruenter plus taril et fendre les opercules.

Certnins npieulteus extrajent le miel in mesure qu'il est récolié jur les abcilles et le muirissent uprès, en l'exposunt ì la chulrur dums des vissenux ouverts. Nous ne conseillons pars d'arlopter cutte méthorie et préférons nttendre pour récolter tout le miel de la récolte en unc scule fois. C'est moing dispendieux, cлi nvec le promier systeme un homme pcut soigner cinq ou six ruchers durant le temps de la récolte et extronire ì loisir uprès, en se fuịsunt aider par los preniors ouriters vellus. Le miel devant entrer en concurrence, pour 


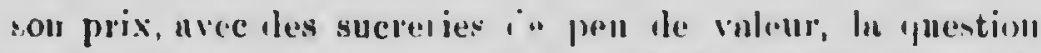

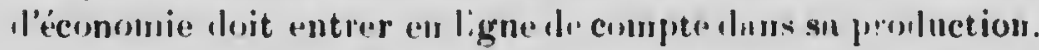

"Celui à qui ln prodnction conteron le plus clrer échonern,

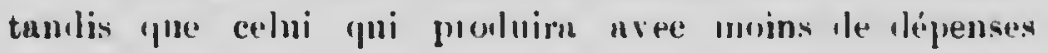
réns:iin"

(.). Hemms.)

Comme certunes colonies we commencent is trasuilet

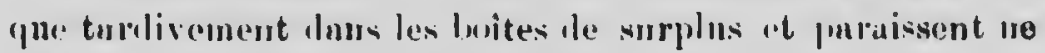
pas pouvoil remplir tomt l'espance qu'our leur a donné, on

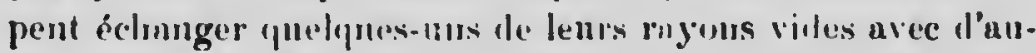

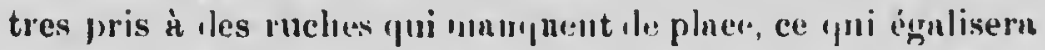

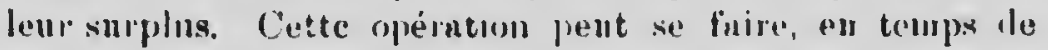
récolte, suns dérnnger les ulwill s pui sont sul ces myous.

Cette égulisntion de monons viles, duns les boitus de surplas des lifferentes colonries, fuite vers la tiu de la récolte, économisera du teups, lors le l'extraction, puce que les boites de surplns serout plus érnlement remplies. En outre, si on donne quelynes loyous de mirl in une colonie qui u'u pus cncore travaillé dans les lwites de suplus, celn l'excite a $y$ monter et stimule sum énergie.

\section{RÉ.COLTE:}

L'extmetion, ponl être fuite rupielement, demunde le travial de quatre personures, troin adultes et ur adulescent.

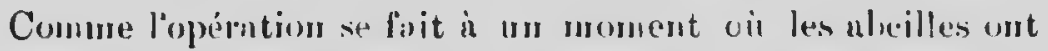
cessé dre récolter, an doit aroir le soin le plus minutienx lo uc pus laisser nne gintte de miel in la poitie des pillardes. Le travail d'onvir les ruclure, de sortir les ryyous, d'en brosser les aheilles loit être fuit tranquil!tuent mus activement. ot soignensement. Les boites où on met les rnyons à transporter doivent être munies d'un convercle, la suche doit être refermée et aroir sun entrée rédnitc unssi promptement que possille. Si on prend ces précautions, le pilluge n'est pas d craindre ; mais s'il commençuit, par quelque uranque de soin on par quelque oubli de l'opérateur, il fund rait urêter 
le trasul jungu'in er quil ait cessé. L'ne eurette d'enu et un eswujemains, plucés à portér, seront trenvés très commodes, surtout si les myons sunt très reniplis, cur l'opérutrur et son aide out furfois leurs iloigts anglués de miel.

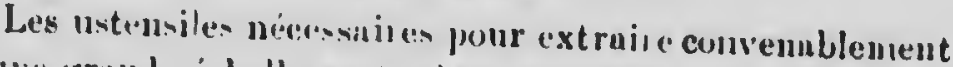
sur wae gromele échelle sont : lans le rucher, un bon enfunoir un ou dellx lulais en hinuclies d'usperges ou en quelque

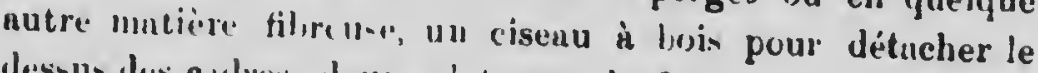
desinus des calles, deux plutenux de f(-1-blane décrits plus bas, une buite is trmaspurter lis rajons et dellx toiles fortes (tuiles i pilleroles) en til un ell r..toln, qui penvent être phemiquée liavance suismut le procelé Rnynor.

C'es toiles ì pillndes, on mienx, contre les pillariles, nomuées ninsi pur le Dr Miller, sunt empleyées à convrir les lioîtes pour ellpécher les pillardes ily pénétrer. ¿́les ont une verge e arrét.

Lopérateur auve me ruche, enlèse in boite le -mrplus,

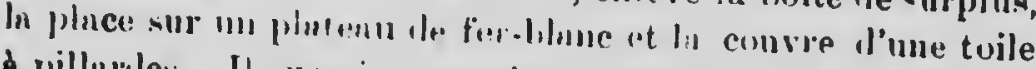

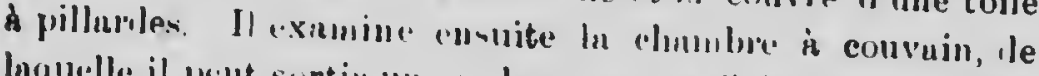

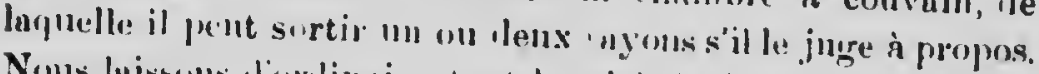

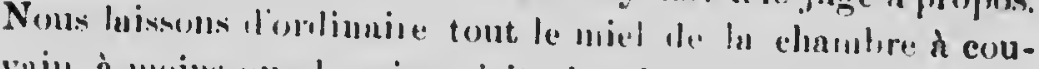

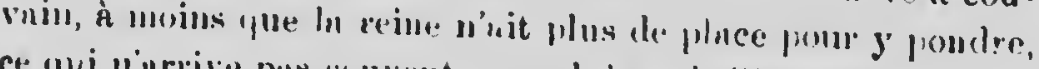
ce qui ninrive pas souvent quunl les nbeilles ont eu ussez de place duni les linusses.

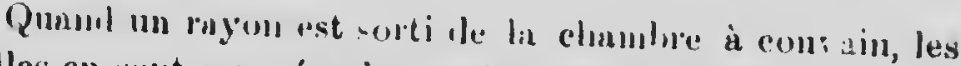
alveilles en sont seconées dromut la ruche ; celles qui restent

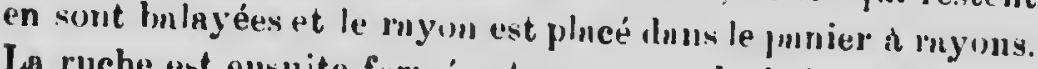
La ruche ent emsuite fermée et une seconde boite de:mplus vide est posét sur un seconil platenu de fer-blnne; pnis les rayous de ln boite pleine sont, l'un npò̀ l'autre, secoués et brossés devant la suche et placés à mesilre dnus la seconle boite. L'aidr, qui est d'ordinaire peu exercé en apiculture, pent être d'un bon secours liuns ces opérntions et si les ubeilles sont muniés dans les règlé, personnes n'ent piqué. Dès que 
les rayons sont $t$, trussrusios, l'nille les prorte luns lu pièce oi) on extruit, tandis fue l'njiculteur prépure une intro colonie, la bolte dont on vient l'enlever lis myons scrunt a transvaser ceux de la huite suivante. Qunnd lu récoltc est abondante on pent se servir d'une lironette pour porter les boltes duns la clinunlore ì miel.

Un américnin, M. Porter, a inventé un petit instrument en fer-lifanc pour finie surtir les ubeilles de leur propre gré des boites de surplne. Un comp d'eril sur ln tig. 62 suffirm

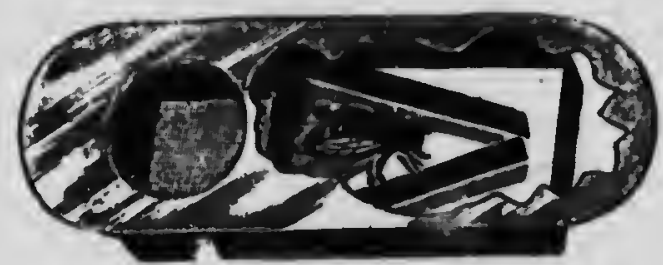

Fig. 62- Chasas-abeillew Porter.

pour expliquer sou fonctionnement. Ce clusst-nheillen est muni de deux ressorts de luito:ı très unince, qui s'écurtent sans ceffort pour lnisser sortir l'aleill 's pui ue ln laisscut pas rentrer. On insère l'instrument ......s un plufontl le 2 ou 3 lignes d'épaisseur, cloué diıns un cudro de la dimeusion exncte de lu buite de surplus II est nicessnire de lnisser au-dessus et nu-dessous du plafond, un expmee oi les nheilles puissent circuler \& l'aire.

Lu boîte de surplus étunt enlevén, on place ce plafond sur la ruche et on remet ln lwite. Quelques nlucilles lécouvrant qu'elles sont enfermées se mettent en quête i'une issue, et l'ayant trouvée appellent les autres par leurs luttements d'uiles.

En pluçnat l'instrument la veille du juur sù l'on doit récolter le miel, ou le matin si on veut récolter le soir, on trouve les loites de st.rplus vides d'ubeilles; a peine en reste-t-il une douzaine duns chaque loûte. láemploi ilı 
chnsae-ubeilles, commo nucun rnyom n'est mis in decunvert et comme on n'a cilteme nheille is seconer on d brosser, evite tont pillage et remil le travil plus ngresuble et plas prompt ; h peine est-il nécessnire l'njunter yue le clinsse-alieilles s'en.

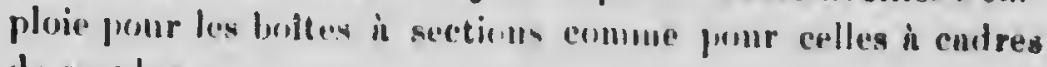
de surplus.

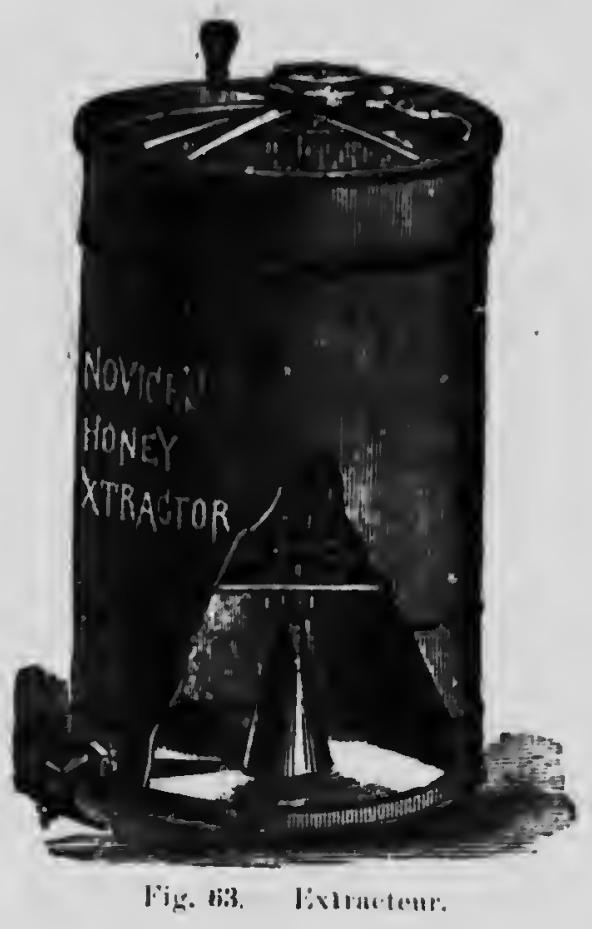

Dans la chambe ì miel un doit avoir un extracteur (fig. 6:3), me cuve a ipercnles, nn entommoir muni l'un crible, un sean de for.hlunc, nn coutenn a désopereuler(tig. 64), un tonmenu et denx platcuux semblables à ceux qu'on emploie nu rnclier. lee pluncher pourru être couvert d'une toile, cirée ou pcirte a l'huile, pour receroir to miel qu'on pourra lasiscet lomber; chaque persuuno devm porter in tablicr le toile peinte orr cirée : et les fenêtres devront etro munies de toile métallique, pour permottre aux abeilles de 


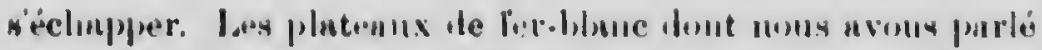

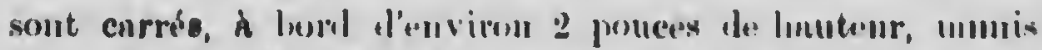

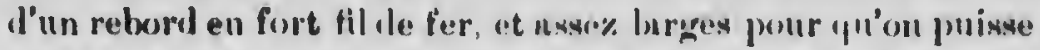
y placer d l'niso uue dles txsites de surplus: ils sont flostinés

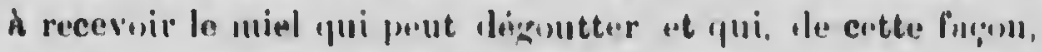
ue salit rien et ninttios pus les pillucies. Ou les munit in clinque extrémité de fortes et larges boucles pmr losifuelles on les transporti.

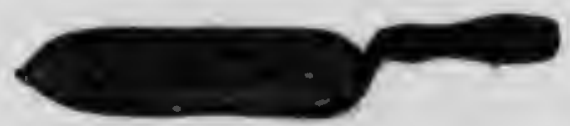

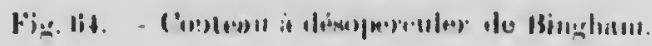

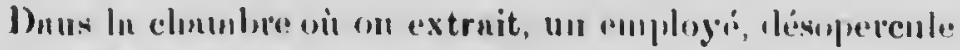
les rigons ì mesure qu'ou les lui apporter. Ponl celn il est

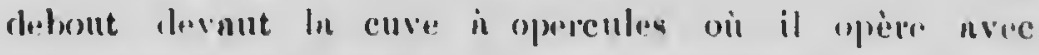

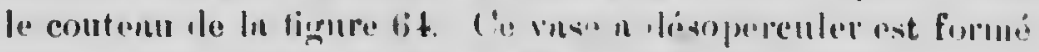

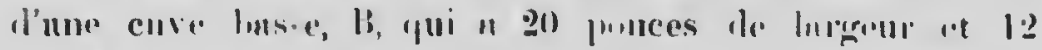
ponces do linuterur, sire

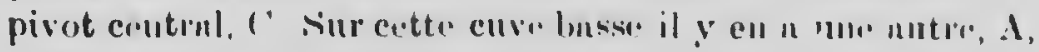
de 20 ponces de luntenr, munie l'un foud an forte toile métullique qui pose sur le pivot ( . La cuve supérienre sert de passoire; nll-dessus al'elle on met un cndre de bois 1), 'pii est entaillé pour qu'il restr à clemeure sur lis lnopls de la cuve. C'est sur ce cnire quion pase les ruyous delwut pour les ilesoperculer: les opercules tombent sur le fond de toilo métallique, d'oì ils s'égonttent cluns ln cuve lu bus.

Les extrnctenrs entièrement finits le métul sont le's seuls empluyés aujourlliui. Ceux ì deoux chulpes sout les plus communx, mais l'on se sert aussi d'extructeurs ì quutre c.udieas.

Quant all coutean in disoperculer, Binglinu (fig. (it), : 1 est réellement co:nuo:le. Ce coutenu vous léharrasse de l'ennui dinvoir les operenles collunt an ruyou dont on vient 
de les rustr, parce qu'il est nuuni l'un bisenu forģnnt le ruseur (employé qui enleve les opercules) à le tenir obliquement, ce qui finit que lis opercules ne penvent pas se recoller an ruyon, à moins qu'on ne le fusse exprès.

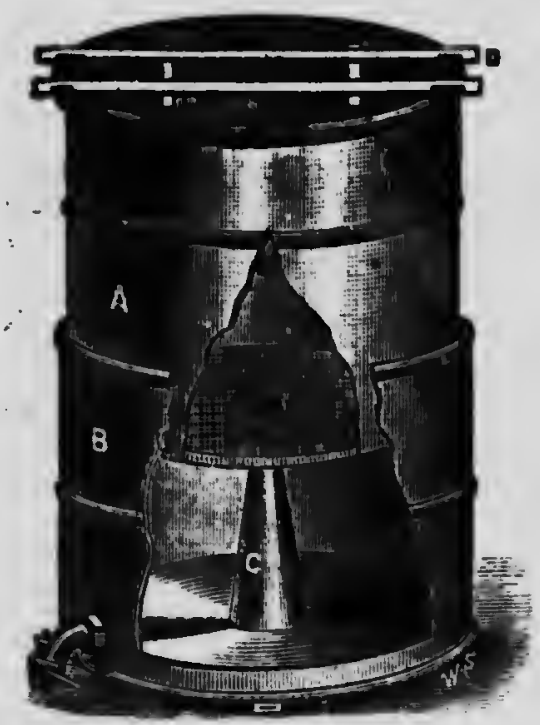

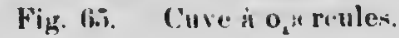

A mesure que les rayon: sont lésoperculés des deux côtés, ils sont placés rluıs l'extructenu', qui peut être tourné par un adolescent. O11 doit veiller à ce que les rayons qui sont vis-d-vis l'un de l'nutre soient, autant que possible, de poids égnux, parce que quund les poids sont inégnux l'extracteur se balance de iroite à goniche, fatigant l'opérateur et la machine.

Une rotation trumpuil/e et régnlière est suffisante pour vider le miel et en temps chand il rast lancé contre les cotés de la muchine, fuisant un bruit semblable \& celui d'une bonne pluie sur un toit de fer-blunc.

Quand les rayons sont vidés d'un côté, on leur fait faire demi-tour, pour les vider de l'autre, M. Stanley, de New- 


\section{$-14.3-$}

York, a inventé un extructeur (tig. (6i)) dans lequel les rayons sont retournés simplenent enl tourıant la manivelle en sens contraire. Des cxtructeurs du mêue genre ont ét6 inventés antérienrenent en Ang eterre par M. Cowan, et des perfectionnements de tonte sorte, lun la même direction ont été étndiés nux Etnt-Unis, snrtont en Culifornic, mais sans qu'ancune maehine, oì lev rayons se retournent sans qu'il soit besoin de les sortir, nit tronvé benucoup de partisans.

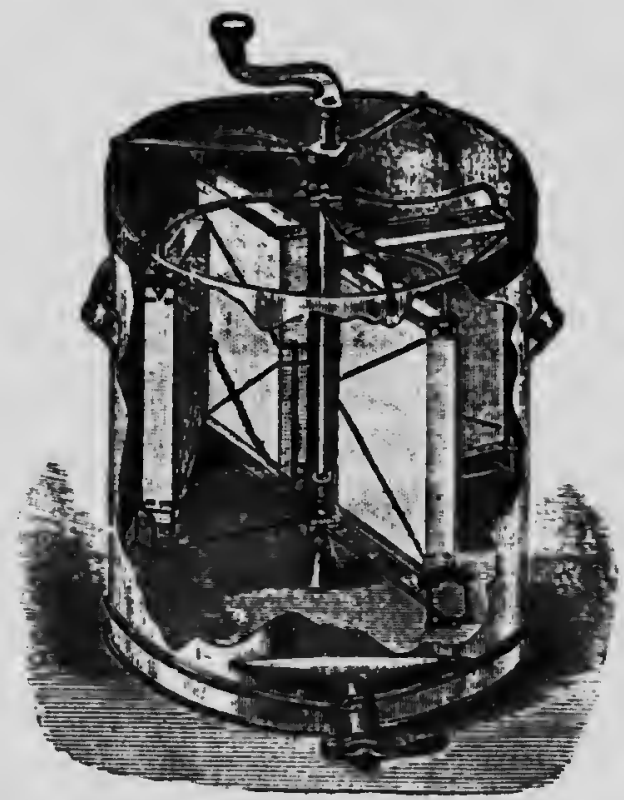

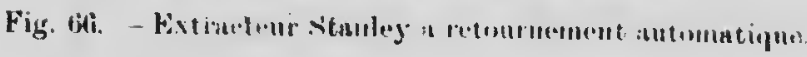

Nous devons conseiller aux commençnts qui extrment pour la premièrs fois de procédor lentement et suigneusement. Un peu de soin nun senlement économise le temp., maig épargne plusienrs livres de mial at renl la elosse plus agréable; car une livre de miel, si elle est repundne, rend tout. gluant et malpropre, tandis an'un trouve rlu plaisir dans une belle récolte et une extraction fate avec propreté; une chambre enduite de miel i.t de's aleeilles en colère dans le 
rucher irritent et l'upiculteur et ses nides, 'pui sont bientrit déguntés de la besogrre. Qunn! tout est bien condnit, le travail est si "gréuble qu'un tronve nisément plus d'onviriers qu'on we peut en occuper.

De turtes les opérntions d'upiculture, cest l'extruction qui demmude le plus de précautions contre le pilinge. Il fuut éviter soigneusement l'exposer des l'ayous ou dı miel; non seulenent les pillardes ennuient l'upicuiteur, mnis elles excitent les ubeilles, qui se fâchent et piquent.

'Toutes les hoites, dans lesprnelles on a replncé les rayons vidés, sont empilées sur'la toile ciréc ou sur des p'ntenux de fer-blanc los rnyons ne doivent jamais être rendus nux abeilles avant le soir, quand le soleil est conché, pour prévenir toute excitation lans le rucher. Eır une lemi-lieure, tous les ouvriers y ailant, les hoites sont remises sur les ruches, mêm. qunnul on aurnit extruit plus de deux mille lives ell un seul jour. Naturellement, si on s'est scrvi de plafonils à chnse-nbeillec, il fnut les enlever avant de replncer les boîtes.

Il y a des saisons oì me petite continuation de récolte permet de rendre les rayons aux abeilles ì mesure qu'ils sont ridés. Leur retour, dans ces circonstances, ne cuuse pas d'excitution et se fuit plus nisément.

Les abeilles ire mettent jas plus de deux on trois jours après l'extruction, pour nettoyer les rayons et les réparer. Mais, pour iviter les ravages des teignes, nous les laissons sur les ruches perdant tout l'été ; les nbeilles en prennent soin Pour l'hiver, nous empilons les boîtes fermées avec soin duus des chmubres saus fell, oir le froid de nos hivers détruit les axufs de teigne qui pourraient s'y trouver.

Dans les loculitéx on il y a deux rècoltes de miel distinctes, chaque réculte doit êtı recueillic stiparément. 
Il est importmut, quund on offre dn wiel in l'épicier ou au consommateur, de le présenter suns une forme nttrayunte. Le miel en rayou, en sections du poids l'une livre seulement, est celui qui se vend le mienx, étunt un urticle de fantnisie. Muis une boite de miel extrait ne pesant qu'une livre est trop petite. Nous levons, pour encourager la consommation, dépenser le moins possible pour le vinse daus lequel nous livrons le micol; or une boits: de fer-lilane ne contenınt qu'une lise nuguente le prix nu point que cette petite quantité est ici de moins ell moius deman lée.

I.es hoites qui cuntent le moins cluer pour loger le miel

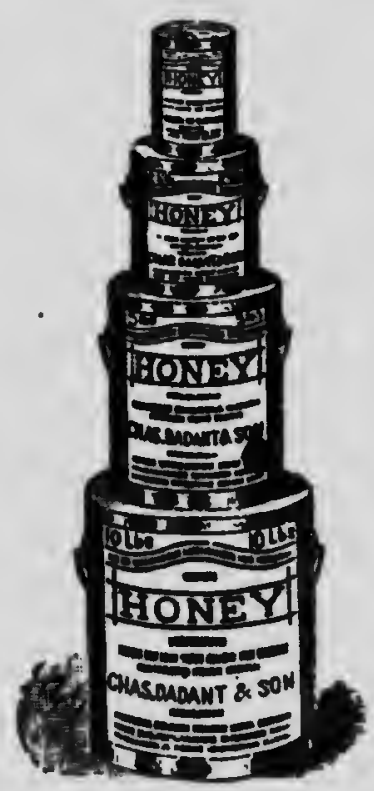

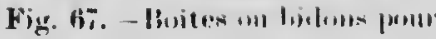
micl extrait. extrnit qu'on clétnille en petite qunntité sont celles de fer-blanc. Les granileurs que nous préférons. contiennent deux livres et demie, ciuq livres et dix livres (fig. (i7). Celles de deux livres et demic sont les plus cournutes.

On loge le miel en futnilles nu moment de ln récolte, nu lieu de le mettre inméclintoment dans les brites. Car l'on ue sait pas d'arnuce dnus quelles proportions se vendrn chaque giandeur et aussi muce que le miel ainsi logi occupe trop le place, n'est pas nussi aisément tannsportuble d'un endroit a l'nutre, et entin purce qu'нyнnt |purfois à gulder du niel d'une lannée d'abondanes jusqu'à l'unnée suirante, quii peut être paurre, le miel se conserve mieux dans de grosses futnilles. Si vous devez garler du miel penilant deux aus; les boîtes peuvent se rouiller en dehors 
et en dedmns et devenir invendnbles. ('omme on peut en juger, ces oljections sont très importante-, surtout pour le grund prorluctenr qui ॥ des dizaines de mille liv à soigner. Qunnt un petit prorlnctenr, il peut mettre de suite son miel en petits colis tout prèts pour le détail.

Pour urêter les fuiter qui peuvent quelquefois se produire dans les boites de fer-blane, qui sont livrées a bas prix par les ferbluntiers et qui laissent passer le miel clans des joints à truvers lesquels l'enu ne coulerait pas, l'on frotte simplement de cire mêlée de snif l'endroit qui coule. L'on empêclie aussi le miel liqnicle de déborder, quand on l'enroye en été, en trempant avont de la remplir, le hord de la loíte dans de la cire mêléc de suif. Le bord extérieur est ainsi garni d'un petit cercle de ce mé!nuge qui rendl ln join. ture fermée hermétiquement.

I1. Singwart, l'Altelorf, Suisse, offie, dans la Revue intermationale d'Apicultur, des boites en fer-blunc dont les convereles. feumunt par pressio semblent présenter une gan antie clininteménbilité suffisante.

On vend nussi benucoup de miel dans des vases de verre, mais le miel grunulé n'y $n$ pas une belle apparunce et en outre ils coûtent plus que le fer-blanc. Cenx qui emploicnt ces vases de verre fundent le miel nvant de les remplir.

Toutes les fois qu'on sencl alu miel, il doit porter le nom et l'adresse du prolncteur ; c'est un moyen de se faire connaître et une grarnutie de sa qualité.

Fabrication de la cire. - Lorsquon vent extraire la cire le vieux rayous ou celle qui provient de la désoperculation des rayons, snns se servir d'un outillnge compliqué et coûteux, on emploiera le procédé suivant :

A la partie inférieure d'un chaudron, on fait adapter un robinet. Le chaudron doit être placé sur un trépied suttisamment haut pour qu'un arrosoir soit facilement placé sous le robinet (fig. 68). 
La ehrudière, remplie anx deux tiers d'ean, est ensuite placée sur le feu ; lorsịue l'eau bunt, on y ajoutc les rayons, puis, à l'nide d'uu lâton, on brasse le tout jusqu'ă ce que la cire soit entièrement fomlus !

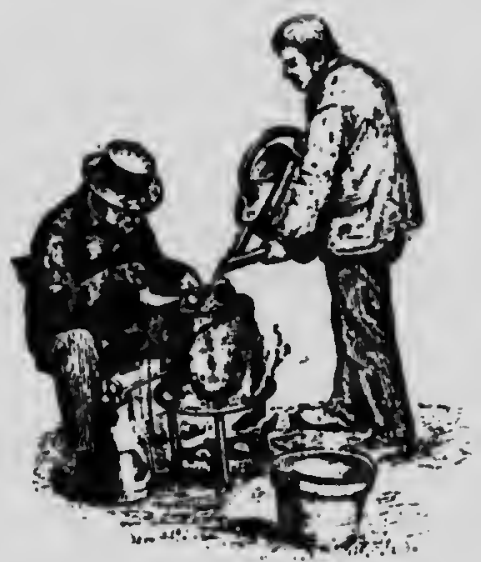

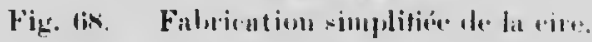

Lorsque la cire est fomlue, on sontire par le robinet l'onu bouillante lans l'arrosuir. A l'nirle d'une pussoire de cuisine on puisc dans la clianlière une certuine quantité de marc mélangé de cire et l'enı, et tanılis que d'une muin on soutient cette passoire au-lessus le la clundière, de l'autre on y verse tonte l'eau buillunte contenue lans l'arrosoir ; cette eau entruîne ln cire avec elle et il ne reste duns la passoire que du marc que l'on jette. On recommence cette opération jusqu'r̀ ce que tont le marc dlu chanulron soit épnisé.

Lorsquion a fini, on retire la chaudière du feu et on l'entonre de paille ou de foin en ln courrant aussi de couvertures afin que le refroidissement se fnisant très lentement, la cire s'épure.

1 -On doit avoir suin de ne pars mettre trop de rayons at la fois, et do diminuer le feu lor'sque tout ext ell ébullition, de crainteque la cire en fusion ne délvorde de la chaurière, car ellc est inflammable. On disposera le feu de façon à ce qu'il ne chauffe qup le dessous de la chaudière. 

Europe.

C'est le procédé que l'on emploie le plus souvent en

Cérificateur solaire. - Un nutrc pre sédé pour fondro la cire corsiste lans l'emploi du cérificuteur solıive appelé aussi purificateur.

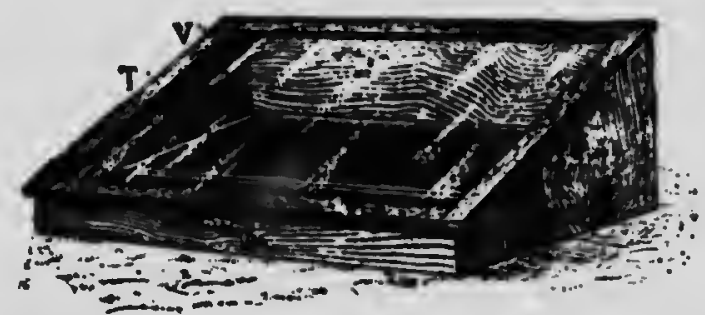

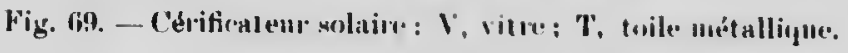

Ce procédé est le meilleur pour ln cire provenant les rayons désoperculés, mais les très viellx rayous fondent difficilenent luns cet nppureil.

Le cérificutenr se compuse d'unc sorte de funpitre vitré (V, fig. (i9) contennnt une toile métullique placée an-dessus d'un récipient en fer-blunc. La cire est posée sur ln toile métullique ('T, tig. 69), ct l'appareil étunt bien exposé au soleil, la cire foml et passe an travers de la toile pour tomber dans le récipient

Fabrication de la cire en grand. - La faliricntion de la cire en grrunl constitne un nrt spécinl qui uc suurait être lécrit duns cet onsruge. Cette fuluricution exige nu mntériel qui coûte fol t cher: presse ì extraire ln circ, chnuelière, épurateur, moules, etc.; de plus, les npèrations nécessuires pour obtenir de la cire partaitement épurée sunt asse\% compliqnées et demantent un loug upprentissuge.

S'il se trouve par hasnol que l'apiculteur possèle une grande quantité de cire, il sem plus simple qu'il In vencle un cirier que de la fo:ılre lui-même. 


\title{
('HALITRE IIXIFiSL:
}

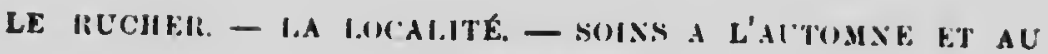 \\ IRINTEMIS.
}

Qui peut "woir des abrills's

Toute personne, nimnnt les abeilles, peut réussir en apicultu:e. Les dames elles-mêmes, moyennant un peu d'rido a certuines époylues, peuvent gouverner un rucher important. Nous pourrions citer les nons de plusieurs dumes des Etats-Unis qui réalisent de beunx protits avec leurs abeilles.

Presque toutes les locnlités peıvent, en unnée moyenne, donner un surplus de récolte. Eit M. Holterman, le Brantford nous disnit, nprès avoir parcouru une partie de la Province de Quél,ec, qu'il était certrin que tonte personne, voulunt soigner intelligrmment des abeilles, pourrait obtenir de benux risultats.

Cependant il est indispensuble que ceux qui désirent faire de l'apiculture sur une grande échelle connaissent les ressources mellifères de ln contrée; car tandis que les abeilles placées dans une localité ramasseront, de vastes provisions, d'autres, $\grave{k}$ quelquies milles de là, ne trowveront pas asseź pour donner grand protit à leur propriétaire.

"Lorsque Huber hal,itait Cour, et plıs tard Vevey, ses abeilles souffraient tellement de la rareté des fleurs qu'il était obligé de les nourrir pour les conserver, tandis que des ruches à trois inilles de là récoltaient alondamment. "

Ceux qui désireront faire de l'apiculture une affaire só- 
rieuse verront le sujet de ln locnlité traité plus loin nu chapitre de la flore mellifère et le nombre de ruches que peut comporter un teriitoire.

Les lébutunts ell upiculture trouveront très-rurement qu'ils ont gagné d̀ commencer sur une large échelle. En employant la ruche d cadres, i's pomront nugmenter rapidement le nombre de leurs colonies, s'ils recounaisseut non seulement qu'on pent gagner le l'argent uvec les nbeilles, muis qu'ils possè lent les cnpucités requuises pour réussir. 1 u, tandis que de gr ınds protits penvent être rénlisés par les apiculteurs soigneux et expérinnentés, ceux qui na possèrlent pas ces qualités sont à peu prèi certains de voir leur mise de fonds produire pen ou rien. De tontes les industries le la campagne, c'est l'upiculthre pui demnule le plus le suvoir faire, nussi pent-on l'appeler me ntfinire de létnils, innis en revanche, elle pent donner le plus de protits nets.

\section{SITE IIU IRLCHFK}

'Toutes les fors ylion établit $n$ rucher, il finut aroir grand soin de protéger les ruches conitre les grands vents. Les abeilles ne doivent pus être plueées clans des lienx ou elles pourrilient être inquiétées par des passants ou des animaux, ni près des encluits on des clevenux paissent ou s'arrêtent. Si on lris.e les ruches essnimer naturellement, il est désirable qu'elles soient en vue de ln urison ou au moins asse\% près pour que le hruic de l'essaimage puisse être entendu. Il est lion qu'il y uit près de là quelques arbres pour que l'essuin n'aille pas trop loin Leur entrée doit être tournée dn côté llu sul, lu surl-e-t, ı) du sud-ouest.

La place occupée par le rucher peut êtıe garnie de gizon, qu'on faucheru souvent pour que les ubeilles ne soient pas gênées dans leur vol. Du sable, du gravier, des cendres de houille, etc, répandus au-ılevant des ruches, valent encore mienx. On place trop souvent les abeilles dans des endroits où elles peuvent périr; soit en tombnit duns la poussière, 
soit en se heurtant contre les herbes d'vin les crnpunis et low arnignées les guettent.

Chnque ruclic uloit atre placée sur un platenu sépuré et entièrement in lépcurlunt des autres, et laisser nutunt 'pu. possible, entre elle et ses voisines, un espace suffisunt your que l'npiculteur puisse fuire le tour de chncnne d'elles. Les rungées de nos ruches, dit Dulunt, nont \& trois verges l'une de l'nutr environ et les ruches dans le rang sont environ deux verges l'une de l'autre, de centre centre. Chaque colonic se trouve ainsi complètement isoláe, de sorte que nous pouvons l'ouvrir sans avoir craindre d'être piqués pur les abeilles de ses voisines. Cet espace empêche aussi Jes abeilles ile se tromper ie ruche.

Ces listances ne sont pas absolument inrlispensubles, m ii; nous ne saurions trop conseiller d'espucer les ruches, même s'il étuit nécessuire de diminuer la place occupée par le potiger, car c'est là qu'on les place généralenent qunnd on ne fuit pas l'exploitution des nbeilles sur une grande éclrelle.

\section{RUCHERS COUVERTS}

Lees rucliers couverts, en générul, protègent peu les ulwilles contre les extrêmes de chnul et de froid, tundis qu'ils angmentent les risques le pertes le reines et de jeunes ubeilles. Los plus fuibles colonies sont toujours celles qui en perilent le plus, cnr leurs jeunes onvrières, en revenant le leurs premières sorties, sont nttirées par le bruit et le inouvement qui se font nutour les ruchées fortes qui les nvoisinent et confiment le proverbe 'que l'ean vn tumiours à ln rivière en se joignnnt elles ınêmes nux plus fortes ruchées.

Iesrucliers couverts sont commms, surtuut en Allemngne et en Itılie. Leur principale qunlité est de protéger les ruches contre les voleurs qu ınd on peut les fermer ì clef. Maisces constructions, sirtout quand elles ont plusieurs étagen, conviennent pej pour les ruches à plafonl mobile. Les ruchers 


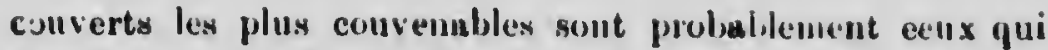
sont de simples abris, tonrués an sud et ouvers durant les grandes chaleurs de l'été et les jonrnees clınules de l'hiver. Les constructions à plusieurs étnges, dnns lesiquelles les ruclus souvrent dans tuntes les directions, n'ont menne valcur; leur seule qualité étant'd'être un ornement con̂teux.

Pour In fucilite des unanipulutions, les rucles placéex a l'air libre sont les mieux situéres. Eil éts, rien ne curvient au-si bien aux atieilles que l'ombrnge des arbres, s'il n'est pas trop épais, et si les branclies ne sunt pus asse\% lunsses pounr gêner les abeilles daus leur vol.

Quand les ruches ne pellvent êtré éloignées l'une de l'uutre faute d'espace, il est endispensuble de les peindre de cunleurs différentes. La planchette qui sert ì rétrécir les entrées pent a ln rigueur suffire, si elle est d'une coulear différente d'une ruche à l'autre.

John Mills, dans un live publiée is Londres :- $18(i 6$, donue les avis suivants: " N'uubliez pas de peinlre les entrécs. de vos rilches de différentes couleurs telles que rouge, blenc, bleu, jnune, etc., en forme de croissunt ou ell enrré, utin que les ubeilles puissent mieux reconmuitre leur logis".

\section{ACHAT E'T TRANSIOHI'P IES ABEILLES}

Le commenynut tronvera eertninement qu'il sera plus fucile, pour peupler son rucher, l'ncheter des essuims de l'nnıée, évitant ainsi, jusqu'à ce qu'il y soit mieux préparé, les perplexitér qui accompagnent souvent l'essaimage soit naturel, soit artitieiel. Qunnl on nelıète les essnims, $i ;$. ut qu'ils suient grus et précoces, sinon on doit se préparer à iles pertes. Si on acliète de vieilles colonies, il faut les ehoisir bien peup'ées et bien portantes. Si on les trunsporte au printemps, après que la saison du travail à commeneé, it vaut mieux quielles vienuent l'un rucher él ignéd'aı ınoins trois milles. 
Si, lorsqu'om vouldrn enporter les ruches, les nbeilles no sont pas tontes rentrées, on lancera un pen ile fumce par l'entrée pour que celles qui sont $\lambda$ l'intírieur so gorgent de miel et n'essnyout plus l'aller nux climmp. On répetem l'oprirntion de temps en temps et en une demi-heure presifue tonies les nheillos soront revenues si quelpues-unes s'étaient aroupies h l'entrie, In fumpe les murnit.. lientst chnswées end dedums de la ruche.

In meillenre snison pour acheter des colonies est te printemps. Il fnut choisir un jour fruis pour le trmusport. parcerplelles sont mons disposées is sortir.

On pent aujourd'hui ncheter de hounes colonies il'Italiennes ell rnches in endres pour un prix inisommulde.

Si les moyens de l'uchetenr sout limités, s'il regurde d la lepense, ou s'il veut employer un nutre mo lèle le ruche qño celui dans lequel sent les nleiller, il pourrn acheter tes abeilles communes lans des ruches in rnyons tixes, mai: il fern lien de les transvaser au plus tôt et de les italimiser. Ces manipnln" "ons domeront nu novice ln pratipue qui lui

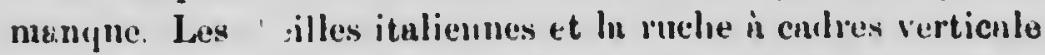
sont selon $" x$, nn nombre des meilleurs gnges de succès.

On ne unit ucheter nucune colonie à moins pu'elle nit du consinin is tout legré de lével ppement pour prouver quieile n in: leime en boume sinté.

\section{AIAMESTATION WR.S ABEIII,KS}

Il y a peu lle soins en mpiculture qui soient nussi nécessaires que l'nlimentution des abeilles qunnd elles en on besuin; cependant rien n’est plus négligé.

L'apiculteur, un printemps, ne doit pas plus négliger de nour:ir ses nécessiteuses que d'approvisionner sn table. Le retom de In chaleur du printemps stimulant la ponte, les abeilles dépensent beancoup dr nourriture et in grund nonıbre 


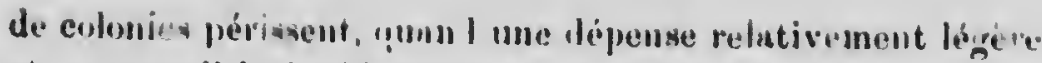
et un truvail inxignitinut lov an rnient sanvées

11 eat lon de remnrquer ici jue ce n'est pas tan! l.

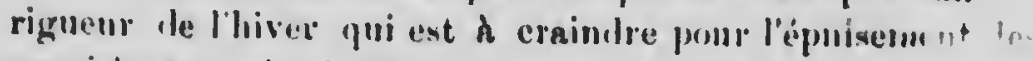

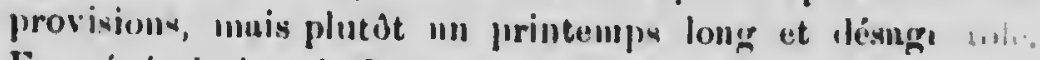
En générul duus la Province de Québece, la trunsition a "tre I hiver et l'été est ns.sez, courte,plus courte que sons less clim. to plus temperéeses la vegétation se léveloppe plus rapicles..entr

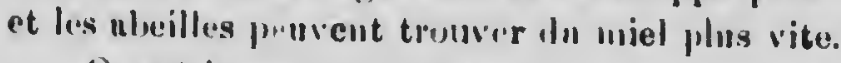

Qunnt ì ceux qui refusent de los nourrir upres une w 1 .

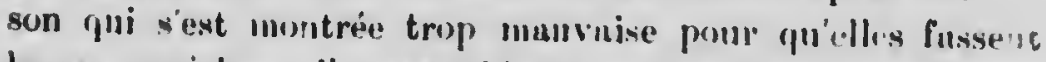
leur's provisions, ils ressemblent anx marchumls qui bot's rnient lenrs vuissennx un retour a'un voyngr infructurix.

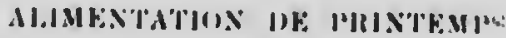

Dès que les utueilles commencen: à surtir an printemps, il est hon de les nourrir quelque peu, cill une légère uddition à leur ordinuire excite la production du conving. (n deva cependant prendre les plus gran les prienutions pour ue pas canser le pillnge et pour celu, on fera lien de ue donner In nourriture que le soir. Anssitôt qu'on verru les uheilles rapporier un peu de miel, il fnudru cessor, car si nne culonic est trop monrric, les nbeilles, en remnlissnnt de miel les ruyons \& ouvrières, gênent la ponte; de sorle qu'il unnit mienx valu jeter le miel que de le leur distribuer. Cet excès de nourriture tonnée anx alieilles ressemble par ses lésultats aux influences nuisibles sous lesquelles sont élevés un trop grund nombre d'enfunts riches; habitués quils sout $h$ se rassasier ou plutôt à se gorger de nourriture, trop sollvent la richesse n'est pour enx qu'un déwainantage grave qui les conlluit de chute en chute et avant l'n̂ge \& une mort certaine (Dadant;.

Les colonies qui ont des provirions suffisantes penveut etre excitées à élever du convaín en enlevant on grattant 
simplemeut les operenles qui conv rent lo uniel de lems rayms. Ce uniel mis ninsi h léconvert, engugenut los onsrières $n$ nourrir la reine plus nl,',ndnmment, nuguente sn puntr.

Les abeilles penvent nvoir lesoin l'être murries meine qunnd les chumps sont couverts le Heurs nimut liancerture de ln récolte principale, si le temps est lifinimalile is la proluction du nectur. Le convenin "pui éclot clinqun jumr en

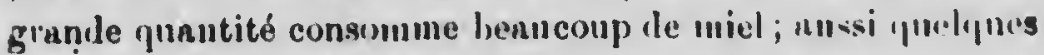
jours de disette, uêne ì la veille llune cueillette ulnmilunte, purvent mettre en danger In vie des colomien.

Le mcillenr moyen do nourrir les colonies manjunnt de provisions all début lu printemps est de lenr domner les rnyọns de miel, si un n pris la précaution ilent réserver yuelques-uns en untomne daus ce but. Si on ne pent empluyer ce moyen, on peut mettre ln nourriture, iniel ou sirop, lans les cellules l'un ruyou vide qnion plucern a la purtén ln groupe que forment le's ubeilles.

1)u miel co partie granulé pent être lonné en petite qunntité à ln fois, en le versunt sur les rayons, mu-desisns du groupe que forment les alecilles. Plusieurs d'entre elles seront inonlées et si dles étnient loin lo lenr lubitntion ellas présenternient 1 II triste spectucle; mais che\% elles, elles se lèchent l'une l'uutre avec untunt de pluisir qu'un enfunt. lèche ses loigtu enduits de contitures.

si une colonic posede trop peu d'abeilles, il seria lon de lui en njouter avant le la nourril. Ce ronforcement de fribles colonies avant le nourrissement demande plus dre soin et de jugenent que quelyue untre opérntion l'npiculture rfle que ce soit. Cenx yui emploient ln ruclie à culdes inubiles ont rarement besoin de recunrir à ce moyon, qui ne pent réussir que quan! tontes les circonstunces concunrent à nuc production rnpide le consain. 
NOURHISSEMENT U'AUTOMNE:

A l'éperpe oì ln récolte du miel cesse, toutes les colonies doivent être populeuses et, en snisons friounbles, leurs pruvisions dloivent être suffisantes pour qu'après une répartition égale clnque colonie se tronve munie l'nsse\% de nourriture. Si quelques ruchées en unt trop, l'nutres trop pen, rien do plus facile que de les égaliser nu inoyen des rnyous mobiles.

Dès que la production du miel cesse, í l'urrivée des temps froids, toute l'alimentution doit s'effectuer promptement. Si on la lifférnit, les ubeilles n'ayant plus asse\% de temps pour operculer leur miel, il' se chargernit d'hmmidité, pourrait aigrir et leur domner ln liarrhée.

Divers nourrisseurs - On fubrique et on vand des nour rissenrs de toute uspèce. Dans notre opinion, un

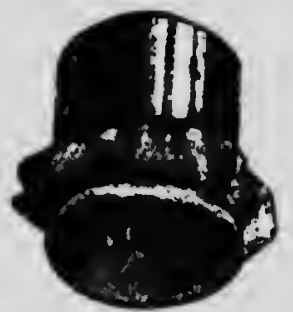

rig. 70.

Bichu|-|lentrisent: lon hourrissenr est un simple bidon de fer-blanc, counme celui dans lequel on conserve des fruits (fig. 70 ) Ce lidon tient ¿ peu près une pinte. Quand il est rem. pli de miel ou de sirop, on lie sur son ouverture un linge d'une texture convenable et on le remverse sur un plat. Le liquide s'en échuppern quelque peu d'aborl, mais In pression atmosphérique l'arrêtera bientît; on le portem renversé près de ln ruclue pour le placer l'ouverture en liss, snns le plat unturellement, sur les carles, au-dessisus du groupe des abeilles, ln toile étant sunlevée à cet effict et le chapiteau convrant le tout. Les abeilles peuvent ainsi unettre cette provision en magasin sans s'exposer au froid et l placent dans un endroit convenuble pour s'en servir plus taril.

Voici la description d'un appareil ussez siuple, appelé nourvissnur, disposé pour les ruches à cadres. 
La figure 71 représente ce nourrisseur. C'e-t une buite en fer-blanc où on introduit le sirop par l'ourerture ", qu'on ferine par un louchon $b$. Cette boite se place renversée sur les cadres. Dans cette position, le nourrisseur présente alux abeilles une surfuce $p$ percécs de truus, asse\% petits pour que le sirop ne s'écoule pus, et à truvers lesquels les alieilles viendront puiser le licjuide sucré.

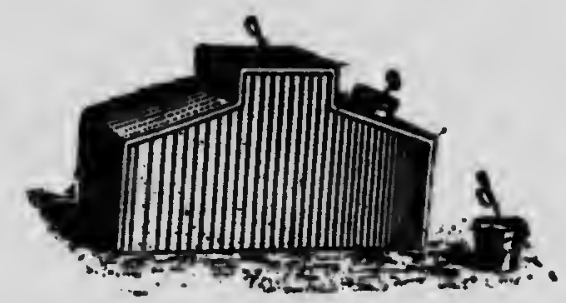

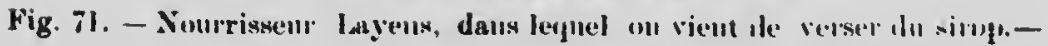

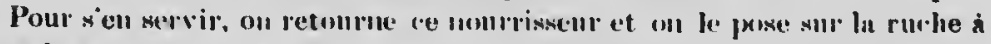
eadres : 0 , oritice par. lequel on t verse le sirop et quion Inonche avec le

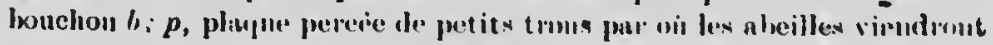
prendie le simple.

Il est préférable de faire l'opération vers le suir ; le lendemain, le nourrisseur sera ville

Il existe bien d'antres sy-tèmıs de nourrisseurs.

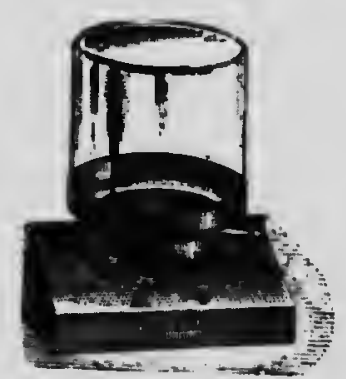

Fig. 7. - Nourrisum Ragnor sim. plifie. - Wu tourn unt le nourriksen. nur lu:-onême, on pent graduet la quantité de sirup dimunie.

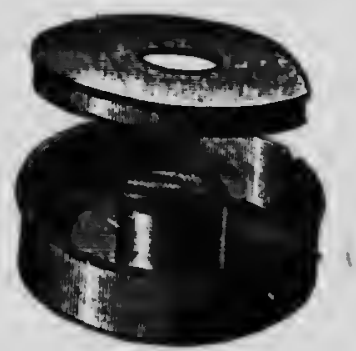

Fig. 73. Nonrrisseur aluglain. en for-ubluwe, à rylimle intérieure. 
Le plus simple de ces appareils est le nourrisseur a bouteille renversée. On place dans une ruclie une bouteille remplie de sirop et on renverse cette bonteille pleine dans une petite auge en fer-blunc; on incline plus ou moins la bouteille, de fuçon que le sirop ne se renouvelle dans l'auge qu'n mesure que les abeilles le con:omment.

On peut encore citer les nourrisseurs anglais tel que celui que représente la tigr. 73, le nomrisseur Raynor, qui perinet de mesurer ln quantité de sirop donnée (fig. 72).

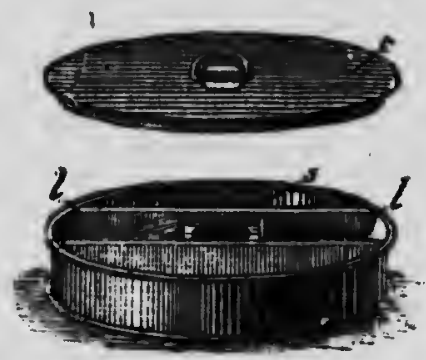

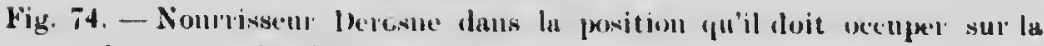
rucle : s, une des denx parties latérales on l'on verse le sirop; $l$, l', lanes sur leqquelles les abeilles viement se umurir; c, convercle.

Il y a encore l'excellent nourrisseur de Miller. Il peut contenir 25 livres de nourriture et sert surtout pour les colonies qui n'ont pas de provisions pour l'hiver, ce qui est très rare.

Il est basé sur ce principe qu'un liquide versé dans deux vases communiquant, prend dans les deux le niêne niveau. Le liquide passe sous une partition et les abeilles, pour $y$ avoir accès montent par le passage pour atteindre le sominet sous le couvercle. 'Le grand nvantage di se nourrisseur est de permettre de passer directement de la chambre d couvain, dans le réservoir.

Comme ce mole de nóurrissement force a décourrir le dessus des rayons, il est bon de donner le sirop avant qu'il soit refroidi. Il échaufte ainsi l'intérieur du chapiten'1, tt 
les ubeilles en mouvenent entretiennent cette chuleur et vident plus vite les bulons.

Joe nourrissements d'automne cloit être fnit aussi promptement que possible. Si on le tranuit ell lonirueur, les abeilles excitées consommeraient duvantage en élevint lu couvain.

Les nourrisseurs de bois présentent l'avantage qu'un "'a pus lesoin de les sortir pour les remplir, mais ils ne peurent pas être mis nussi prèz du groupe des nbeilles. Il est inutile de roppeler que le nourrissement doit être fait le soir pour prévenir le pillige, surtont qunnd on emploie du miel.

II. Scholt\%, de Silésie, recommande la prépnration suivunte :

"Prenez une chopine le miel et 4 livres de suere blanc écrasé bien tin; clunuffe\% le miel, sans y njonter d'enu, et mêle\%-y le sucre en truvillant bien ln masse pour In remlre homogène. Couper-ln en tranches minces ou divise\%-In en moreenux, que rous entourere\% l'un linge in tissn pen serré nvunt de les mettre entre les myons on sur les condres. Ja plasticité de cette pâte permet ì l'npiculteur de la donner de la façon qu'il préfère. Les abeilles ont uruins de ditticulté à employer cette corte le nourriture que le liquirle et il y a moins de perte."

Cette préparation a été employée depuis quelques années avec succès aux Etuts-Unis, comme nourriture pour enroyer des reines par la poste et pur le chemin te fer, sous le nom de "cundi de Good". Je sirop épnis et le candi sunt sans contredit les meilleurs succédnnés du miel pour nourrir les abeilles qunnd elles doivent être continées pen. 4. lunt longtemps et n'ont pus de miel.

Une expérience de $M$. de Lnyens $\Omega$ montré que les abeilles peurent employer de l'eau pour dissoudre le sucre. Le même écriłuin raconte comment un apiculteur français, M. Beuzelin, nourrit ses nueilles en hiver. 
"Il scie un pain le sucre en trancirs et place ces tianches nu-rlessus des cnlres sons une toile. Un nutre upiculteur m'u lit, il y a quelques nunées, noir sanvé des colonies en ruches de puille en y suspendant simplement, au moyen de tils de fer, des morcenux de sncre pesant plusienrs livres."

Ces méthorles, qui rénssisstut dans des climats inodérés et humile: comme celui de France, ne convientrnient pas dans le nord les Etuts-Unis, encore moins an Cnumla.

Comme règle grenérale, il ne fnut uvoir reconrs in l'untre nourriture que le miel, q qu'ell ens d'absolne nécessité. Dès lors, il funt lone toujours garder quelques myons pour fuire fuce unx besoins impreivns.

Linpiculteur prodent doit considérer le nourrissement des abeilles, ì l'exception du pel ju'on domne an printemps pour stimuler ln ponte, comme un mul auguel il fuet arnir recourx seulenent quend on se peut l'éviter. (Dadant). 


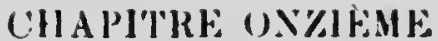

\section{IIVERNEMENT}

Las ubeilles peurment supporter I liver solls tuus less climats, à ln condition 'plue l'été $y$ lurern ns-(\% longtemps pour leur permette do rnumssel des provisions suffisuntes pour l'hiver.

Anssitat que le tempis froil nrive, les nheilles sins-

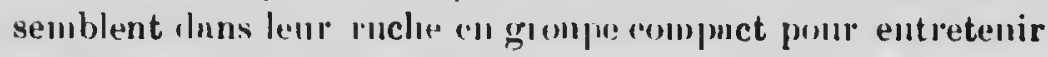
leur chuleur. Elles ne se rémissent pas sur les myons remplis le mirl, mnis sur les cellules villes nu-dessous du

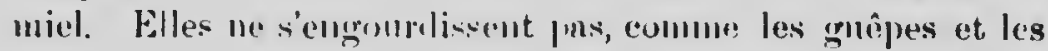

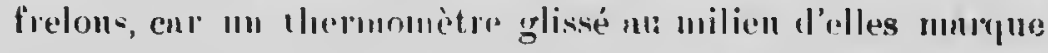

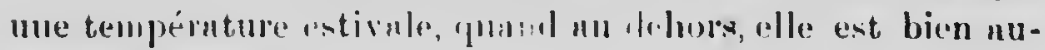
dessons de gerr.

Les ubeilles, en gronpe, sont imblinuées enume les tuiles l'un toit, clacunc "yant la tête sons l'nblomen cle celle qui est immérlintennent nu-rlessú, et ninsi de snite, jusqu’à celles qui peuvent utteinle le micl ; crllesci en passent ì celles qui sont sous elles, qui, il leur tour, en doment allx saivintes

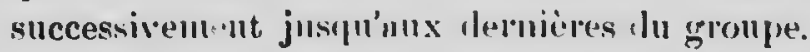

Qunnd le froild devient rxcensif, elles s'agitent par des vibrations continuelles, pour alevelopper une plus grande quantite le chuleur par cet "xucice * et in mesur. que celles

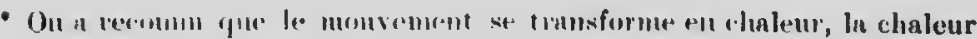

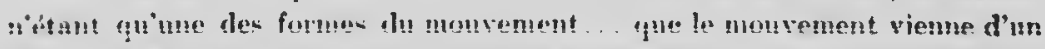

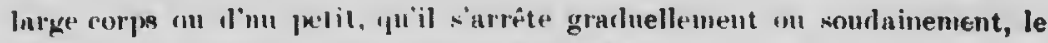
résullat ext le mima, il ast tmosformé en chaleur.

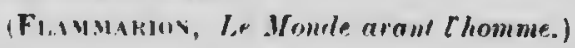


qui øecuprent l'extérieur du groupe se refroidissent, d'autres les remplncent. En ontre, le battement d'ailes envoie, du sommet lu groupe nu bas de la ruche, de l'nir qui réchauffe les uleilles placées a lu partie inférieure; ct celles-ci, si elles ne sont pas trop engourdies, protitent d'un jour moins froid pour griuper uu-lessus de la masso et prendre du miel a leur tour.

Qunnd le temps est très froid, on peut souvent entendre leur bourlonnement de l'extérieur de la ruche; et si, qunnd elles sont grompées, on frappe sur leur ruche, elles répondent par un murnure qui dnre plus ou moins longtemps at dont le son est plus ou moins intense, suivnnt la force de la colonie.

Comme tout exercice musculaire a besoin de nourriture pour compen-cre lu dépense du système, plus les abeilles seront trunģuilles, moins elles mangeront. Il est conséquen:nent ties impontunt de les gnrantir nutant que jossible en hiver cutre une grmule élévation on un grand alaissement de tempernture, qui, tums deux, les excitemient à beaucoup l'activité.

Qunnd toute la nourriture qui est à leur portée est consommée, elles périssent si lu température est trop froicle puır leur permettre de transporter leur groupe entre d'autres rajons contennut du miel. Il en résnlte que lorsqu'on met les abeilless en hivernage, si les ruyons du centre de la ruche ne sont pas bien garnis de miel, on doit les échnnger contre d'autres plus remplis; utin que, quand le froid force les abeilles ì quitter les myons extérieurs, elles puissent se russembler sur ceux qui sont le mieux approvisionnés. C"est surtout duns les régions froiles, où les abcilles récoltent peu de miel d'nutomne, que ces précautions sont nécessnires.

II l'est guère possible de prévoir combien une colonie dépensera de miol pour passer l'hiver en bol état. Celn dépendra beaucoup du système d'hivermage, car on peut li laisser en plein air, ou la placer dans un local spécial où les 
abeilles n'éprouvent pas d'excitations soudaines ni de changements atmosphériques sérieıx. Célu dépendra lieaucoup anssi de la longueur des hivers, qui varient grandement sous les differentes latitndes, et de la précocité du printenıs. Dans le Canada, les abeilles souvent ne récoltent rien pendant plus de six mois: tundis que dans les Etats les plus au sud elles sont rnrement snns récolte pendant six semaines. Dans tous les Etats-Unis du centre, si on les liverne en plein air, chnque ruclıe doit avoir all moins 20 livres de miel; dans notre pays 30 à 35 lives ; mais plutût 35 livres que :32.

Juns les ruclies मे myons moliles, une simple inspection suffit pour se rendre compte des provisions. Le poirls des ruches n'est pas toujours un criterium infuillible, car les vieux rayons sont ilus lourds que ce.ux qui ont été bâtis depuis pen et sont sourent remplis de pollen.

Les apiculteurs expérimentés recommaissent ordinaire. ment d'un coup d'inil le poils des provisions. On doit se rappelir qua les alieilles ont besoin de nourriture, non seulement pour pusser l'hiver, mais aussi pour se nourrir et nourrir leur couiain pen lant les jours froids du printemps, avant que les fleurs donment du nectm: Les ubeilles ne gaspillent pas leurs provisions, et les colonies dont les magnsins sont bien fournis seront d'ordinaire les plus fortes et les mitux préparées pour la récolte suivante.

Les conmençants enthousinstes sont portés à abuser de l'extracteur, laissant parfois trop peu de miel pour l'hiver duns la chambre à couvain. Si les abeilles ne sont pas trop encombrées de mial, nous conseillerons de lrisser aux fortes colonies toute la provision que leur clanmbre à couvain contient.

Il est pronvé qu'il y " du bénéfice à laisser pour l'hiver. aux fortes colonies, une grande quantité de miel, afin qu'elles ne limitent pres leur ponte du printemps. 
la qualité de la nourriture est mi factenr importunt pour le succès de l'hivernnge. Une lungue série de jours froids force les ubeilles i munger alon lammuent, remplissant leurs intestins de untières fécnles lont elles ne peuvent se débarrasser, cur les abeilles ne se vi lent jumıis dans la ruclıe, à moins qu'elles me soient continés trup longtemps uu trop dérungées.

Une nourriture mnlsuine lurant une réclusion prolongée, leur doune la diarliée tît uu tard, non seulement quand on les hiverne en plein air, unis en crive on lor'sgu'un lex envoie ì de longues listunces.

Des expériences numbrenses ont promvé quinne nunrriture sucréte, ties pure, sera celle qui produira te moins d'excréments duns leurs intestins. II 'וl lésulte qu'us miel aqueux, insuffisamment évaporé on nighr, lle mîne que tout

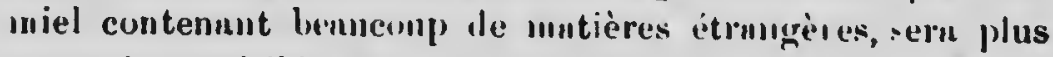
on moins misible aux ubrilles enfermées. I de miel foncé, comme celui de la lingère, contenant me grmule qunutité de

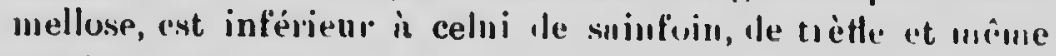
nu sirep de sllere.

\section{R'HIVERSEMEN' E.X II.F.IS Alle}

L'hivemenent en plein nir n'est pas recummunduble sous notre elimat oi des froils riguoneux et de gran tes varintions de tempinature se font sentic pendunt llices: II ne fnut jumnis perdre dle rue yhe pour que les aleilles hivernent bien la tempérnture duit rester mssi cunstunte que possible duns les 40". Or, in moins le premble do gandes précuntions, il est impossil,le l'ol,tenir ce résultut en plein nir.

Cependint comme certnins npicultemrs ont fait l'essni de ce systène soit parce qu'ils n'arnient pus de coves, soit purce qu'ils roulaient se livrer in des expériences, mons allous exposer ici ce que nous royons de mienx in fuire, tout en 
répétant qu'il ue funt avoir recour.s à l'hivernng: en plein air qu'en cas d'impos-ihilité de faire autreınent.

Qunn I les ruches n'out an'une épnisseur de plunches tunt nutour, on doit avoir ginud noin de les nbriter des vents pénétrants, qui épuisernient ln chuleur vitule de leurs halitants; cur, semhlahles uux lumuins, s'ils sout à l'abri du rent, supporteut une busse tempérnture leenncoup uieux qu'un conrant l'air moins froid.

Dnus quelpues pays de l'ouest des Etnts. Unis, oì le vent du norl fuit lenticonl souftirir les nbeilles, on protège les ruches eu hiver avec iles buites de prille pour les défendres contre le froil on l'humirlite. Les feuilles seches et tontes espèces d'herbes sèches conviennent hien nussi. In neige elle. même peut servir, pourvin que les dégels et gelées successifs n'enpêclent pus la ventilation. On ioit, dès la prolubilité de l'arrivée d'un jour clımul, dégnrnir l'entrée le ln neige qui l'obstrine.

Pour mieux protéger les ubeilles hivernées en pleiu nir contre les influences de la tempémture, on a inventé des ruclies à parois iluules, entre lesqualles on place dez matières mauvnises conductrices de la clmleur. Eilles sunt généı lement des mo lifi:ations de la ruche Langatroth is doulbles parois.

Après noir essayé co système on n reconnu que ces ruchées trop lourcles sont difficiles \&̀ manicr, surtout si elles contienucnt dix on onze cadres Quinigy, ou lenr équivalent en calres Isngstroth. Pour obtenir les avantages des ruches do doulic: arois sans leurs inconvénients, en employant les ruch à simple paroi, des apiculteurs ont imaginé de se servir le boites, dont on recourre les colonies durant l'hiver ct qu'on enlève au printemps. On remplit l'intervalle entre la ruche et la boite de: matières peu serrées. feuilles d'artires sèches, ce qui procure ln ineilleure protection (fig. 75). On remarquera que ces boîtes sont fuciles d d6cloner et à empilcr sous quelque nbri quand on ne s'en sert 
pas. Elles out un inconvénient cepermlant, c'est qu'cn ne peut guère les empêclier l'être un refuge pour les souria, les insectes, etc.

Si les colonies nont popul'uxes "l bien "pprovisionnécs si elles ont cles mutières alsorbrinles an-dessus de leurs rayons, un passage facile de rayon ì rayon, du miel mur. un ubri contre les vents fovils, elles réunissent loutes les conditions nécessuires pour pusser l'kiver en plein air.

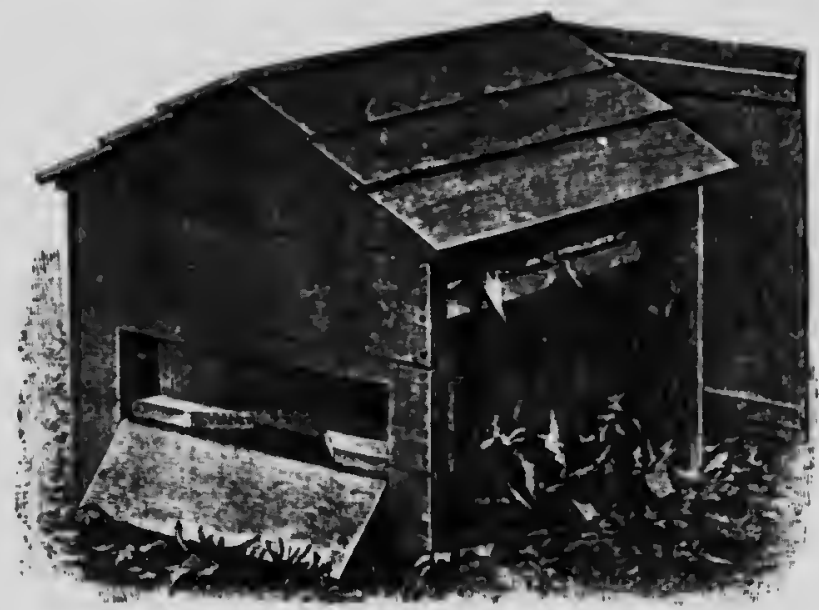

Fig. is.

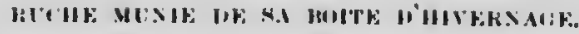

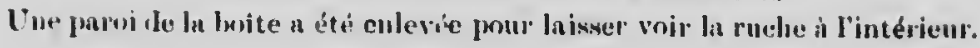

Au lieu le mettre uno fouble boite sur chnque ruche, on a nussi pratiqué le système de réunir les ruches 4 pır 4 en les pluçant dos à loz, denx regariant l'est et les deux antres l'ouest. Par ce procédé l'on diminne considèrublement le nombre des double boîtes.

I) fuut assurer une bonne ventilation et pour atteindre ce but, l'on place le patites pièces le bois de 3 pouces a l'avant et de 33 pouces à l'arrière entre chaque ruche et son plateau. 
Tout en protégeant l'entrée contre la neige et ln pluie par InI petit pont en bois, il fant nvoir soill d'ussurer lo passage de l'eun, de intine qu'en haut, il est bon ile soulever a liaile ilun patit morcenu le bois une corno du paillasson qui recouvre la ruche.

L'on a nussi employé pour empêcher les souris de pénétrer dans l'intérieur le la ruche ou de la double boite, de petits tils du fer piqués de distance en distance devant les prises d'air et $n$ : Inissunt entre eux que juste assez d'espace pur parnettie aux ulyilles de pisser.

Encure une fuis pour la province de Québec, l'hivernago en pleir air qui a pu réussir à quaclques pasticuliers ne nous parait nullement recommunilable.

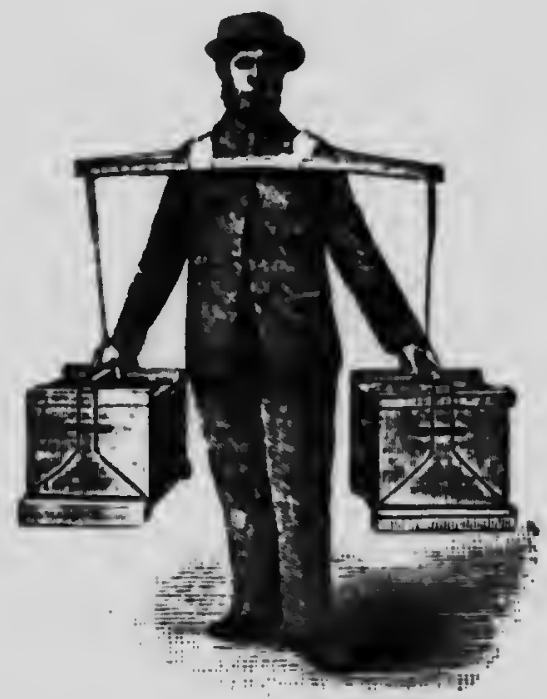

Fiz. ils.-.Transpert des muhes

HIVERAME E.S CAVE

C'est assurément le plus propice pour nos climats rigoureux, car dans une cave bien è̀clie, ll est toujours plus facile de muintenir une tempéruture constunte, qui assure 
comme nous l'avous dejin dit, plus de repos nux abeilles et partunt une moins grnncle consommation des provisions (fig. 7tb.)

On doit mettre les abeilles en core tunt de suite après qu'elles ont ju se vider, nprès une belle jonrmée, an moment oì le temps froill commence, c'est-h-dire vers ie 15 novemlire. Certains apiculteurs ue gardent en hivernement dans In cave que le corps de In ruche, Inissant dans me nutre piece le platenu et le clıpitenu, après mvoir pris la précnution d'inscrire en dedans le muméro de la ruche ", pour pouvcir la remettre ì ln mône plice nu printemps. Disutres, au contraire, hivernent tont le corps de ruclie. I.es denx sys. tènes donnent satisfuctions qunu I les príenutions de venti. laion sont bien prises.

On a da prépurer duns la cave soit des mndriers, soit des boites pour poser les ruches, quion empile les unes sur les untres en les séparant pur les cales. Ces suppoits doivent étre l'untnut plus élevés que le sol de ln cavc est exposé a l'hunidité. On peut lnisser les platcanx uux ruches du bия, mais il fuut les ćlever sur des cales pour qu'elles aient plus d'uération. Os: doit lonuer nussi de l'uir par le dessus, pour que l'humidite diveloppée pur les abeilles s'échuppe ni sément. Si la cave est hmmille on aura quelques ruyo:ss moisis, si elle eat sèche ils resteront pu excellent étnt.

On doit profiter, ponr les mettre en cave, l'une matinée assez fraiche pour que les ubeilles ne sortent pas, les porter et les poser smus secousses et tenir, penclant l'opération, la cave mussi obscure que possible, ne se servant d'un peu de lumière que juste le templs de plucer chnque ruche.

si l'on rentre les ruches avec leurs couvereles et leur plateau, il fint enlever ln toile cilée et soulcier in ruche au-dlesxus du convercle de 1 pouce en avant et $1 \frac{1}{3}$

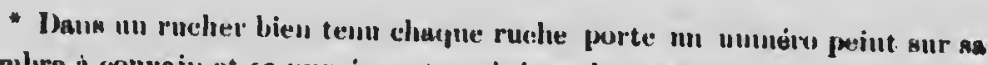

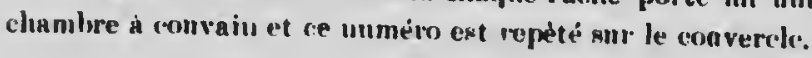


ell arrieste pour donner plus le fiecilitis pour la ventilution et une jógère inclinuisul ell Hvant.

Si l'ou eulieve te courescle, il fint le rempluever pinr un

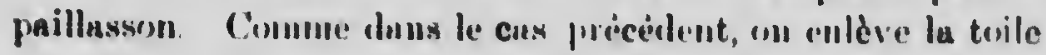
cirée.

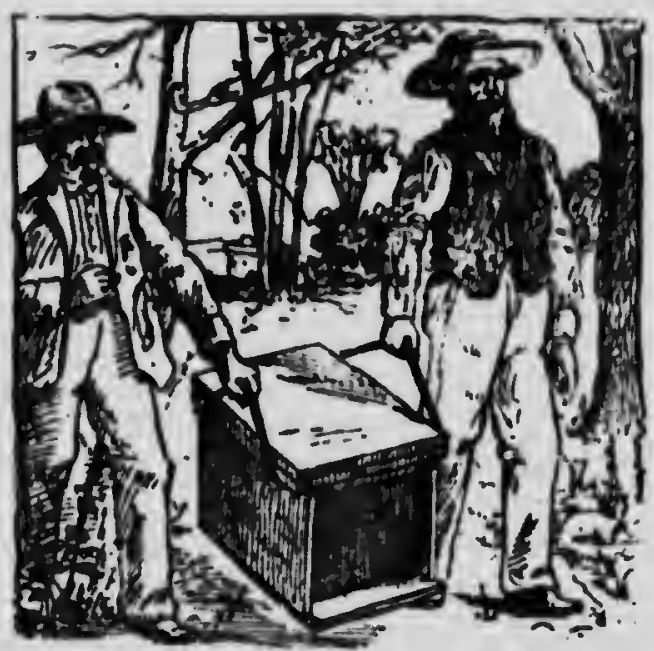

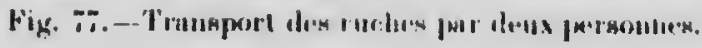

Certuins npicultenrs qui laissent l. platenu soulèvent non senlement la rucle commur dit ei lessus, muis sonlevent aussi un peu le plutenu pour permettre a l'uir de circuler plus lihrentent.

If fint avoir soin de ranger duns votre enve ros ruches duns un oritre déteminé pour pouvirir, en les sortant au printemps, les remettıe à la place qu'elles sccuprient l'année précéllente.

Si vons avez séparé, pour l'hivernement les platenux de la ruche, il funt leur fuire ì chncun quelque signe particulier qui vous peru!ettru de les réunir en temps voulu. Ce sont de petits détuils. muis qui ont leur inıportunce, car en les négligeant, vous rous exposez ì ce que vos colonies se mélangent uu détriment de quelques unex ussurément (fig.77.) 
Quand elles sont tontes placées, il faut les laisser duns une obicurité complète et au llegré cle tempérnture qui assurera le mieux leur tranquilité. Liexperience nous a uppris qu'une températurerle to $0^{\circ}$ Fahr. est celle qui convient le mieux. Qunnl le thermomètre est à ce legré de l'écholle on se clouternit à peine qu'il y a des abeilles dans la enve; si la chuleur haisse ou augmente, le bourdonnement commence. Un themomètre est indispensuble quand on hiverne les alueilles en cave; c'est une alépense iusignifimte et qui permet l'agir à conp sûr.

Comme las nhei!les hivernées en plein nir commencent à volep punul ln chalen a ntteint 5$)^{\circ}$ et sont en plein wol a $5 t^{\prime \prime}$ 'm $5 f^{\circ}$, on pent s'imnginer comlien el'es sont ngities quan, ln tempémtare de la enve s'élève in $\left(i^{\prime}\right)$ : Elles attendent imprtiemment l'mure in jonr ipni leni permettra de voler, et comme le temps pusse sasis que l'obscurité disparnisse elles sont inguiètes et se futign'nut.

Lin untre, la chalemr les axcite in élever ln couvain, et

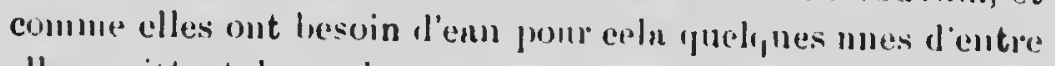
elles inictent la ruch: ponr en charelier et sont porlues. Cer pertes se prorlnisent tonjums quelone pen channe hives. On pent refroilir In eave es ventilant fortentent par nne jomróes séche et froide.

L'apicultenr doit prendre anssi ses précuntions contre le froill mais qunud les colonies sont nombrenses la chuleur qu'elles développent snftit d'ordinnire pour écionutfé suffisaumment ln cave, mề" dans les jonrs les plus froids. Pour permettre à l'air f. .l l'entrer suns luniè re, vici ce que dit Dalant. "Nons a vous imaginé l'arrunger nos volets clung ce but (rig. TS). Quand la fenêlee intérieure, 'ini est snspen. due par le haut, est soulevée, nous mettons ì sa place nu chîssis. Le volet extérienr, qui est tonjours aluissé, n une onverture munie l'une planchette glissant dans une coulisse, qui permet de donner le l'uir san lumière, grâce ì sá disposition extérieure. Nons nons aussi pris ln précaution 
de garnir la fenêtre de vitres doubles: nous la fermon? durant les jours les plus froids et et nous ouvrons la coulisse, surtout la nuit, quand nos abeilles se plaignent de in chaleur une petite quantité d'uir pur venant de l'extériunr étant toujours nécessaire." On peut remplacer ces" portes par des ouvertures de 6 pouces dans les murs, une ou deux suivant les dimensicns de la cave, mais il faut pouvoir les refermer a rolonts.

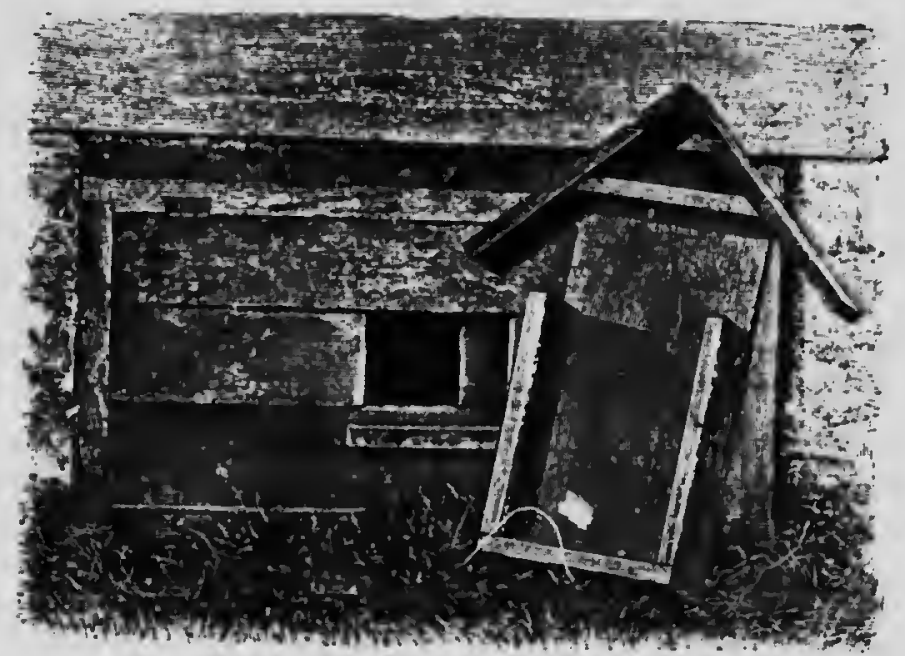

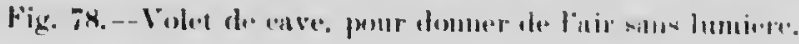

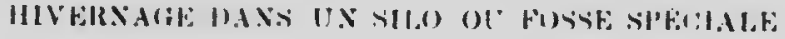

Il est possible que le cuttivatenr qui s'uccupe l'mpiculture u'ait pas à sn disposition une houne cave ou s'il en a une qu'il ha réserve pour ses légumes. Dans ce cns, s'il ne veut pas hiverner ses abelles comme il nété dit nI chapitre: hiveruage ell plein nir, nons allons indiquer un système qui pour être moin's nvantagenx que l'hivernement en cave réussit cependunt. C'est une uxcurntion en plein chnmps dont les murs sont doulles laissunt entre eux, un espace de 4 pouces Le plancher qui est in un pied du sol que l'on n 
eu soin de fonler et aplanir et au besoin de recouvir do cendres on de sciure de bois est égnlement louble mais pleilr.

Les ruchess sout placées ì un pied llu unur sur une double rangie de toiles étendues sur le plancher. Les cunvercles de bois sont enlevés et remplacés par des coussins

Il funt cluvisir un terrain très sec car si l'humidité pénétrait dans la fosse, elle peut fuire courir les plus grands dangers non seulement nux rayous muis aux abeilles. II sernit donc bon soit de construire des murs en brique sur les parois de la fosse et le cimenter. Quant a la dimension de In fosse, elle loit répon/re nux besoins de l'npiculteur. Voici une expérience qui a été fuite et peut nous tixer quand aux résultats obtenus. L' fosse n été creusée sur le versunt d'une colline dont le terrain étnit sec. Flle avait 3 pieds de profondeur, 3 pieds de larg ur et 10 pieds de longucur. Ises ruches ont été placées sur une ligne, laissant aux leux extrémités une place pour installer des ventilateurs. Elles ont été déposées sur deux malriers de cèdre qui courent la forse dans toute si longueur.

Les ventiluteurs, tuyaux en plancliettis de 3 ì 4 pouces, étaient au nombre de quatre. Trois d'entre eux descenduient jusqu'u niven des madriers, le quatrième au contruire ne descenduit pas plus bas pue le sommet des ra. che's. Ces quatre ventilateurs s'élevaient au dessus du nivenu du sol de 3 pieds.

Les convercles des ruches ont été remplacés par des paillassons et les ruches elles-mêues séparées de leur platenu par des inorcenux ile bois de deux pouces carrés pour assurer une meilleure ventilation.

La fusse étnit recouverte de unudriers de cèdre placés duns la longueur inuis de nanière a former vonte. Onavait 
reconvert le tout l'une forte comclie de paille sur larguelle un picd de terre étnit étenclu.

Il est hon answi le se méneger nu centre des ruches un tuynn par loguel on puisse descemlie min thermomitre pour s'assurer, le ln tempérnture an moin: me fris chapue semmine.

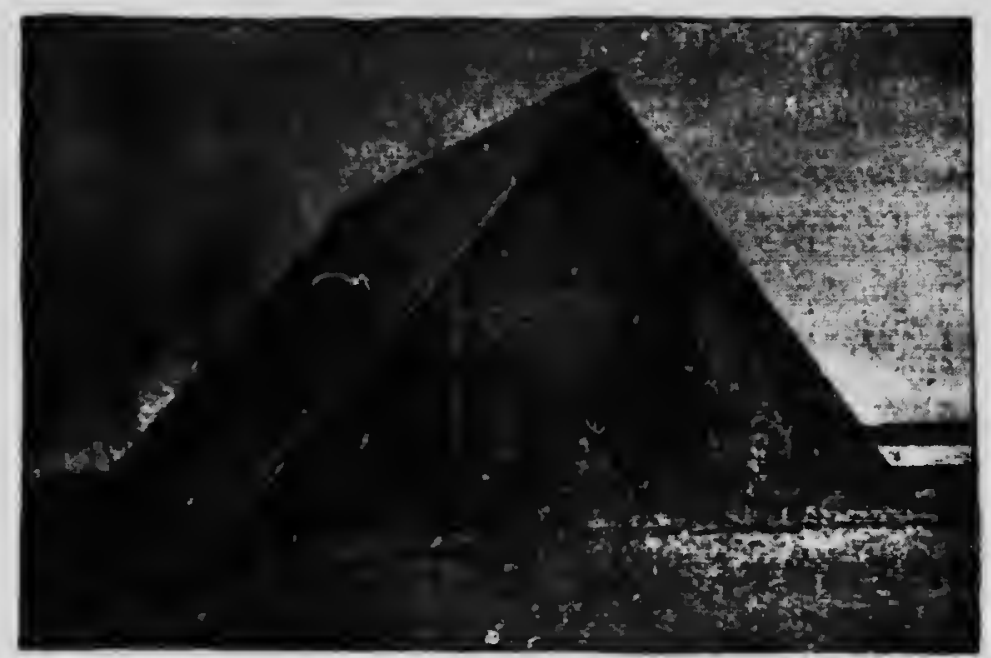

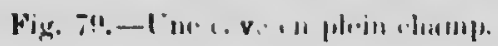

Les colonies peuvent nimsi pusser l'hiver alors mine que la tempérnture lescent is $: 36^{\circ}, 37^{\circ}$, mais encore une fo,is, il

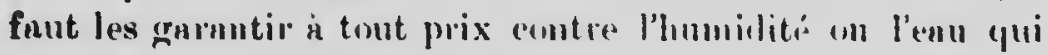
pourrait pénitrer dom la fosse.

Dans som " l, c, sur la culture des al, illr-e, li. li. Rowt décrit en ces termes nue cave all pluin champ, ntilisice ave succès par .II. Binghluill.

Jes gravores (tigr. is-s0) montrent les detnil- de la corso

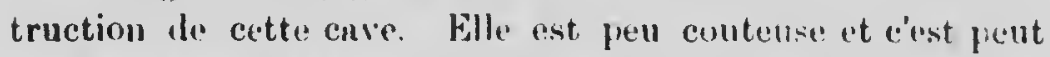
être le meilleur systime lhivernement is l'estín ieur. I. foul le cette cuve a 12 preds de charque crité (an peut e'n fuire de 
toutes dimensions) et les murs qui ont 6 pieds montent en s'érasant_un peu pour !'onner uu sc mu:et 16 picds de côté Les inadriers sur lesquels repose !n partie supérieure ou cabane que nous voyons tigure 79, ont 2 pouces d'épaisseur 12 pouces de lnigeur et 16 pieds de 'ongueur, de sorte qu'ils entourent la cave danx tointe son étendue. Le toît, (cabane) est construit en planches d'un pouce $\frac{1}{2}$ et vient aboutir dans des petites rigoles en deliors les solives, ces rigoles rvant à l'écoulementides eanx de plnie.

Ie plancher au-rlessus de la cave, est construit avec das plunches de 2 ponces d'épaisseur.

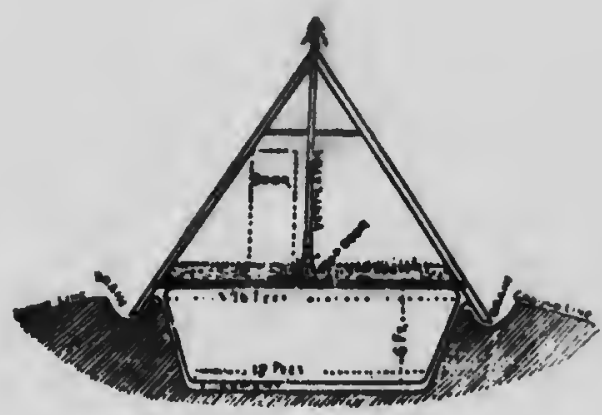

Fig. 811.-- Reetion dle la rave.

On descenrl lans la cave par une trappe faite de bois de la mêne épaisseur que le plancher et un escalier qui va jusqu'en bas. Les nurs et le planchers du fond sont à doubles parois en brigus uu en planche espucées de trois pouces et l'on répand sur le plancher du fond une forte couche de sciure de bois qui la garantit contre l'bumillité.

M. Bingham déclare que cette cave lui a coûté $\$ 55.00$ environ et qu'il $n$ pu hiverner 125 colonies : quand il n'avait que 100 colonies, il ne perduit que 2 livres d'abeilles par nois, ce qui est un succès extraorlinaire.

Il n'avait qu'un ventilateur pénétrant, dans lo plancher 
supérieur de la cave et surtant par le toít de la cabane. Ce ventilateur a 10 pouces de diamètre. Comme on le remaryuera le système Binghan offie cet avantage que lit chumbre d'hivernement est tout entière au-dessons du nivenu du sol ct parconséquent inoins sujette aux variations de température. Dans cette cave, d'uprès M. Binghum, la tempéruture n'u pas varié de plus de 4 legrés. Elle n'est jamnis descendue un lessons de $40^{\circ}$.

Le système de M. Binghum n'ent antre en rénité que celui dont nous arons parlé plus haut; weecette différence toutcfois qu'il possède une chambre an dessus de ln cave au lieu d'un simple toit reconvert le terre La calve Binghrm!n est nussi plus facile d'accè. 


\title{
CHAPITRE DUUZIEME
}

\author{
MALAIIES ET ENYKMIS IOS AHEILLES
}

Maladie de la loque ou pourriture du couvain.La loyur est ln muladie la plus termble qui ${ }_{1}$ isse sévir dans un rucher. On a vu, en Allemugne et en ....gleterre, par exemple, des rucleers entiers, avec de fortes colonies létruits en peu de temps par ce Hénu reduutable.

La loque est une uffection due à un le ces organismes microscopiques qui sont comms imnintenant sous le nom de microhes, et qui léterminent che\% l'homme et les mumanx In plupart iles muladies qui sont contngieuses

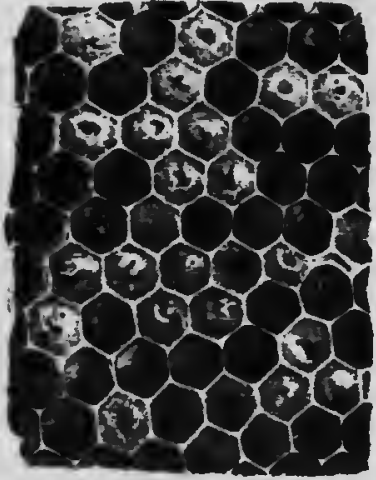

Examiuons un l:y yon de couraiu, et regurdons nate: a:n le comrnin operculé. Si rous voyons des opercules nettement léprinés on percés d'un petit trou, ou même léchirés comme un tambour crevé (tig. 81), retirons nlors la larve de l'uste des cellules à l'nide d'une tête l'epingrle; si nous trouvons que cette larve est trunsformée ell une

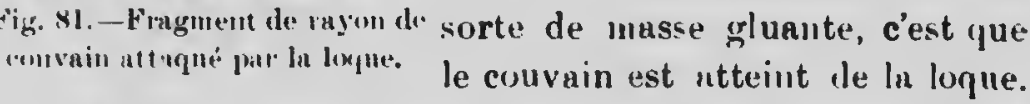

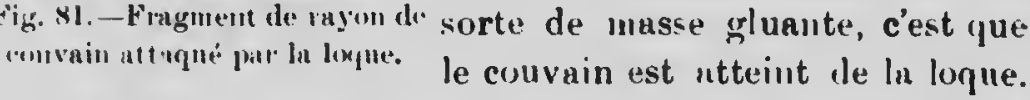
Mais si, è même temps, les larves plus jeunes et qui ne sont pas encore operculées, sont blancher et nncrées comme daus le couvaiu normal, nous pourions ell conclure que la loque n'est qu'à sn première périonle En etfet, lis malaclie. 
au débnt, attaque générnlement de préféıence less larıes qui commencent a s'enfermer iluns leur cellule.

La loque s'eat déechoppée depuis lomglnmps duns la colonie. - Dans ce cas, on peut as-e\% souvent recounultre, Inêne extérieurement, par le travail menti les alueilles at par l'o lenr de pourriture qui s'exhule de l'entrie de lu ruche que la colonie est lorjuense.

En examinnnt un ruyou de curruin, un vit que non seulenent le currain opereule, mais mêne lrs jeunes lnrves sont attaquées par la malaclie; ces deruieres deviennent aussi jaunutres, en s'allongenut dans P'ulvéole, "II lieu I'y prenlre ln forme recourhée habituelle.

si alors, par snite de l'nftinilissement de la colonice, la ruche loq'leuse vient î être pillée, las abeilles pillariles peuvent propiger ln loque daus tout le rucher.

Conditions hygiéniques pour éviter la loque. - Il n'est pas facile de savoir comment ln leque pent at tnquer une colonie. 'loutefois, comme il est prouvé que certuines circonstances fuvorisent lintruluction de cette muladie, il faut éviter autant que possible que cess circonstances se produisent lans les ruclies.

10 Il ne faut pas que le couvain reste lécouvert.

20 Il fuut prendre en tont temus ín plus graudes précantions contre le pillnge.

3. Il fant fomlde les morcens eontenant du couvnin, lors i'un transvasement ou le ln suppression d'une ruche.

Guérison de la loque - La guérison de la loque n'est pas toujums fincile, et lorapue la maladie est arrivée h la périorle la plus grave, le phins simple et le plus prolent serait de supprimer In colonie.

O॥ $n$ inlipué un très grand nounbre de remèles contre la loque. M. Hilbert a été un des premiers d̀ conseiller l'eonploi les antisepitipues, en particulier de l'acicle sulycilique.

$\mathrm{Mi}$, In visite du printemps, on s'aperyoit qu'une colonio 
préselute des signes ds loque à su première rériode, on en fait passer toutes les uleilles duan une nouvelle ruche garnie de myens de cire ginf rée ou de rayons amorcés on a pour ninsi lire raunené ninsi la colonie d l'était d'essaim; cetto opérution doit' être faite ellviron troıs scninınes avant la gruncle rècolte.

1)e plus on aura préraré à l'avarce la soiution suivante.

Duns uneflinte d'cau chaude' on fuit fondre 2 livres de sucre, on $y$ ajoute dix grinımes d'une solution a 12 pour cent d'ncirle sulicylique dans l'alconl. (On trouve l'acide salicrliane dane les pliarniacies): Tous les deux on trois jours, on donnerk a ia colonie une cliopine de ce sirop, et cela pendant trois on quatre semanes.

Si c'est à'la visite d'nutomne que l'on s'apercoit qu'une ruche commence a devenir loqueuse, on $y$ mettra simplement de In niplitaline, et on attendra le printemps suivant pour la ranetuer d l'état d'essain et rour In traiter par l'acide salieylique.

Désinfcotion de la ruche loqueuse. - Il est très importnut Je lisinfecter au plus vite une ruche loqucuse qu'on vient de sil rimer en ramenant la colonie a l'etat d'essaim.

On passe à l'extracteur les rayons qui contiennent da miel. Les luâtizses seront fondues, les cadres seront plongés dans l'enu lou illante ou passés dans une dissolution contenant 10 pour cent d'ucide sulfurique.

Dysenterie. - Cette malarlie se deciare ordinairement penilant l'hivel ot quelinefois in l'Hutomne; c'est une sorte d'indigestion qui se révèle par l'nccumulation des excréments dnns la ruche; celle-ci exhnle ulors nne odeur fétide.

La dysenteric est due surtout ì un hivernage trop prolongé dans un air hunide, insuffisamment renouvelé. Le rellouvellement de l'air dnus l'hivernage, que nous arons recommandé, est donc encore très utilc dे ce point de rue.

Cette malarlie peut venir aussi de ce que l'on a donné ax abeilles une nourriture trop aqueuse; elle peut encore 
provenir de ce qu'elles n'ont pas cu le temps à In tin de la saison d'évaporer l'excès d'eau contenu dans le nectar récoltó tardivement oll dans le sirop qu'on leur a donué. On voik donc que le nourrissement d'inutomne est encore à redouter, pour cette cause.

Si toutefois on observait une colonie utteinte le cetto muladie, on ehangerait son pluteau et on réinstalleruit la ruche sur des cules.

D'ailleurs, la dysenterie n'est pas, en général, une mala. die grave et souvent on la voit disparaitre d'elle-nême au printemps.

Galléries ou Fausses-teignes - Les pipillons, eonnus sous le nom de Gialléries ou Funsers-leignex. sont les seuls inseetes ennemis des abeilles qui puissent eunser des rainges vraiment dangereux. Il $y$ en a deux espèes prineipales; la plus granile est la plos répmidue lons le Noril. et la plus petite se trouve surlont dans les eontrées mérilionales.

Un reuf le fonsse-teigne peut étre pondu sur les tleurs, et les abeilies le rappurtunt, soit avec le pollen, unit avee le nectar, l'introduisent elles-mênes dans la eolonir. Le papillon de la fuusse-teigne peut aussi s'introduire direete. ment laus la ruche th $y$ pondre. L'euf éelot, produit une larve ou elıenille qui a seize petites pattés très courtes. Ces chenilles sont très vives, se tordent comme le petits vers et pénètrent dans la eire clont elles font leur prineipale nourriture. Elle ne se nourrissent pas de miel, muis les bâtisses peuvent être séricusenont nttuquées, et ln mì̀re gênée dans $3 a$ ponte.

An bout d'un eertain temps, les ehenilles se transfor. nent en chrysulides entourées de cocons blanes ut réunies en groupes à côté les unes des autres.

Il sort de ces eoeons des inseetes développés; ce sont de papıllons gri-atres, lont la forme est différente suivant que e'est l'une ou l'autre expèce de Gullérie.

Les abeilles luttent contro les fuusses-toignes. - Si 
une culunie est forte et bien orgunisere, elle ne craint pas les fuusses-teignes. C'est qu'eu effet les abeilles s'nccupent perpétuellement de détruire les larves de Culléries ; ellea percent des trons dans les rnjous envuhis puur en faire sortir les cluenilles qu'elles tuent et qu'elles rejettent au dehors, comme on peut l'observer souvent sur le devnut de la ruche.

Si une colonie finilule ent fortement nttniquée par la fausse-teigue, on ne lui Inisse que les ruyous qui contienunt du couvin, et on lui ujonte des myons passés nu sonf re

Quant uux ruclıes orplıelines, qui tinirnient presque toujours pur être nttnquies, on les supprine comme nous l'avons dit.

Les Guepes, les firelons, les libellules do grande tnille et l'Hyménoptère uppelé l'lilunthre "piorore, sunt des insectes qui cherclient d s'empurer les alveillew. On les voit souvent voler un milieu des butinenses qui sont en grand nombre sur les plantes mellifieres four thclier d'en snisir une.

Les Fourmis sont plus gênantes que nuisibles; celles des petites espèces s'instullınt quelıuefois sous le couvercle de la ruche, surtout à cause de la chaleur qui provient du groupe des abeilles.

A ruchniıles. - Les arnignées attrnpent les ubeiles dans leurs toiles, et sont surtont nuisibles dans les rucliers couverts qui ne seraient pas suffisımmment soignés.

Reptiles, oiseccux, mremmiferere. - Les Lézurds, les C'rapauds et un certuin nombre d'O ivern $x$ inecetivores léts uisent un plus ou moins grund nombre d'aleilles.

Les Mulots, les Sourix et les Blaireuux sont des ennemis plus dangerenx ; les deux premiers sont très communs partout et s'introduisent dans les ruclies, nime lorsqu'ils sont très petits, pour $y$ dévorer tont ce qui s'y trouve et sollvent pour y faire leurs nids. 


\section{CHAPITRE TREI\%IEME}

Examen des ressources mellifères de la contrée. En générul, qunnl on désire se liver à la culture des abeilles on n'est pus mattre le chuisir la ritgion oi l'on veut établir ses ruches, cnr on se trouve fixi prar ses uecupations dans un pays déterminí.

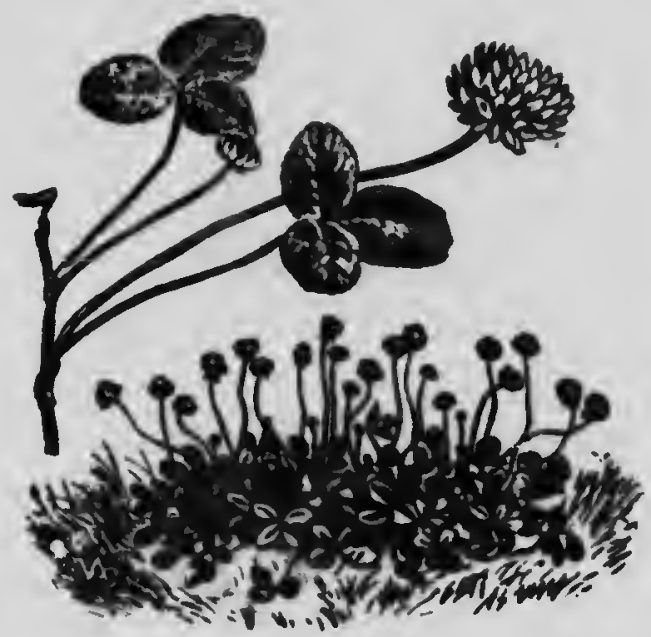

Fig. 8:. - Trètle blanc.

On doit donc se renilre compte avec le plus grand soin des ressources mellifères le ln contrée avant d'y établir un rucher.

Si, cumme ceia arrive le plus souvent, l'empiacement des colonies se trouve indiqué par les dispositions de la pro- 


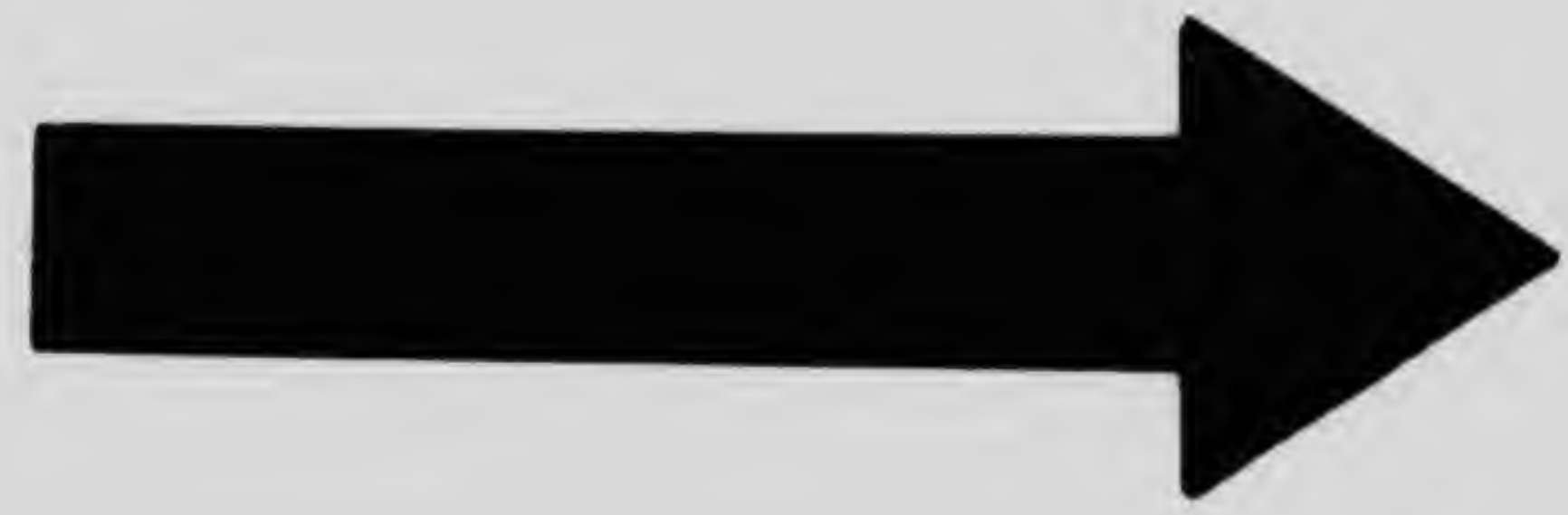




\section{MICROCOPY RESOLUTION TEST CHART}

(ANSI and ISO TEST CHART No. 2)
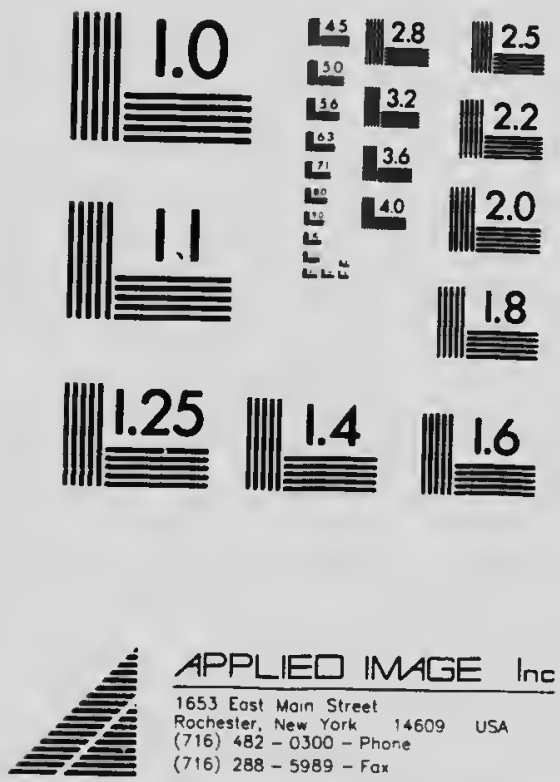
priété, c'est autour de cet endruit qu'il faut examiner la végétation naturelle ou les cultures, en temant compte du climat et de la nuture du terrain.

Comme les abeilles ne s'éloignent quère utilement au delà de deux ou trois milles de lenr ruche, étudions dans ce myon les ressources qu'elle's auront à leur disposition.

Voici quelques indications prutiques, essentielles, sur ce point capitul.

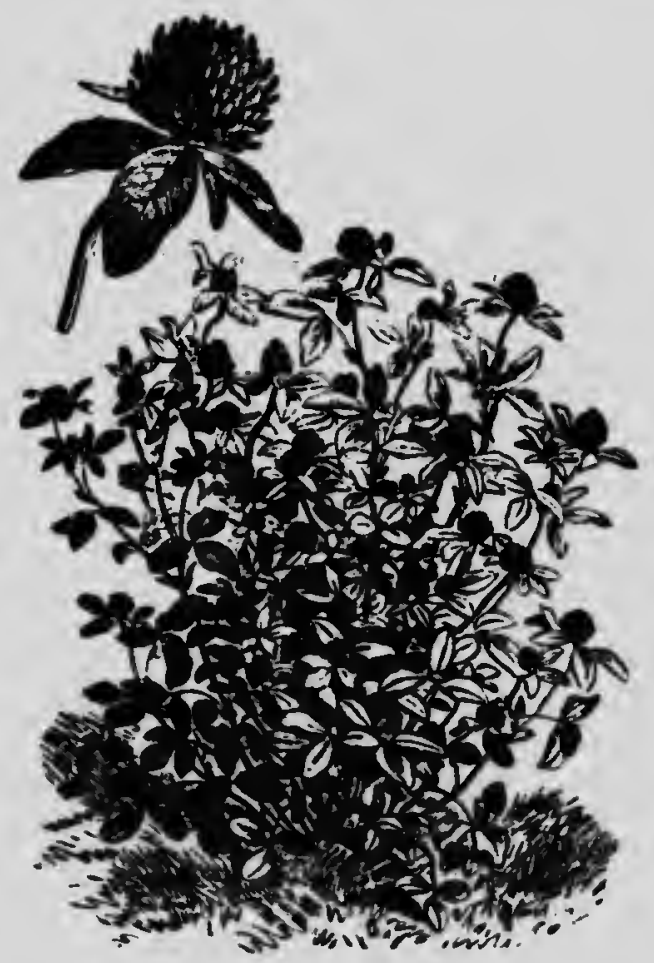

Fig. 83. - Trïtle des prés.

Plantes mellifères de la végétation naturelle.-Sail n'y a autour de l'endroit où doivent être placées les ruches que les plantes de la végétation naturelle, ces plantes seront, dans la plupart des cas, celles des prairies, des bois ou des 
landes. Si les ruches se trouvent plaeées luns une contrée où domiant les prairies naturelles que l'on doit faucher et non dunner à pat 'arer aux bsstiaux, on peut dire: que l'endroit est assez favorable a l'apiculture, surtout si_nnos remarquona dhis ces pratries des plates telles jque le "Trèfle blanc, la Sauge des prés, la Jucée, le Mélilot, le Serpolet, ou la plupart des plantes des prairies, si l'on est duns les montag:ıes.

Lorsque ces pluntes sont très abonduntes, les prairies que l'on donne ì pâturur nux bestıaux peuvent même offrir une res:ourees importun ${ }^{2}$.

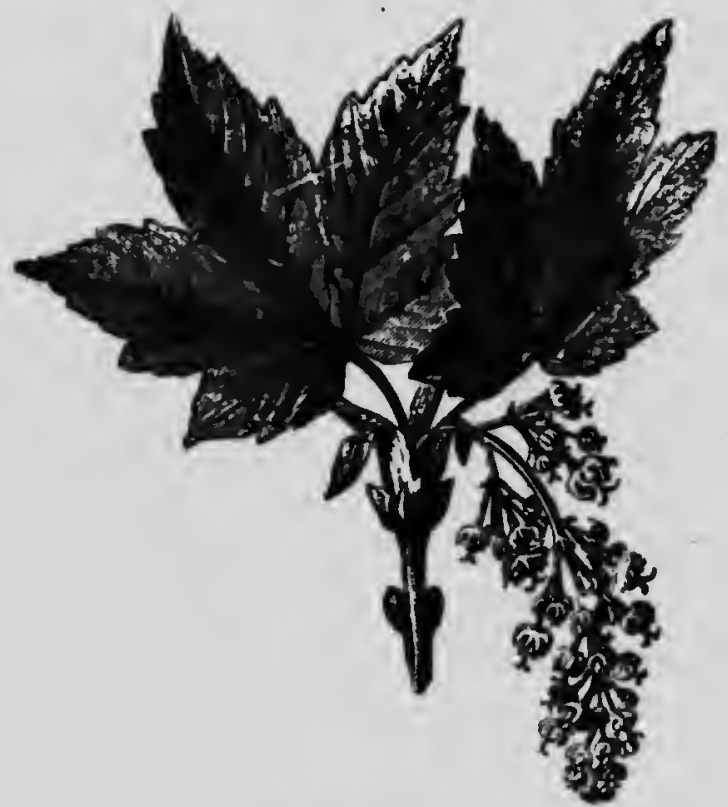

Fig. St. - Hiralse.

Les bois ont l'aruntuge le ronner penclant toute la saison une récolte qui permet presque toujours uux abeilles de faire leurs provisions d'hiver; mais cette récolte est souvent peu abondante, et le miel est le médiocre qualité. Cependant, si nous voyons benucoup de lruyères dans les bois ceux-ci peuvent présenter à l'nutomne une ressoutce importante. Toutefois, il funt rumarquer que ie miel de bruyère 
a le double inconvenient l'être de qualité inférieure, et d'avoir une consistance trup épaisse pour punvoir étre retiré par l'extructelur.

Dans ces régrions, il est lon le fuire In récolte du miel avant la floraison de la hruyère et de laisser le miel de bruyère comme provision l'hiver. Nous n'uroms que peu de bruyères au Cunada.

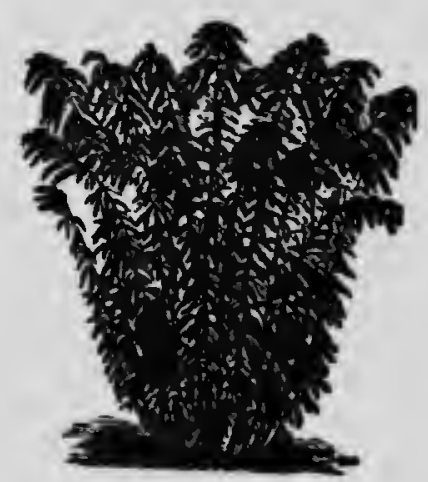

Fig. 8.5. - Verge d'or.

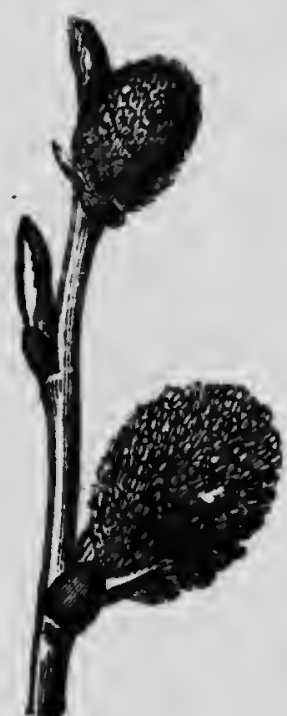

Fig. Nit. - Saule.

Les principales resseurces des bois sont les plantes des lisières et des clairières. Centaurér, Pulmc: aire, Verge d'Or, Germandróp. ete.! 't cortains aubres ou arbustes printanniers tels que les Sunles, lis Merisiers, les Pruniers sauvages, les Erables les Chênes les Acrcias et surtout le 'lilleul.

Les enliroits incultes, les champs en friche, les talus de chemin de fer sont souvent envuhis par une végétation qui renferme des plantes mellifèrcs telles que la Vipérine, 
l'herbe st-Jenn, les Chnrilons, les limnires, la Menthe, etc. 1

Plantes mellifères des champs et des prairies artificielles. - Si les ruches sont un milieu des cultures, il test très importunt l'exnuiner quelle est la nature des plantes cultivées.

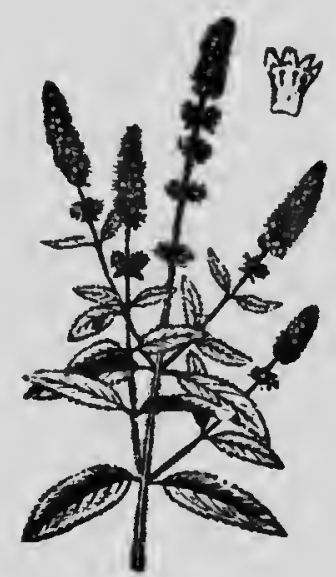

Fig. 87.-. Mcuthe.

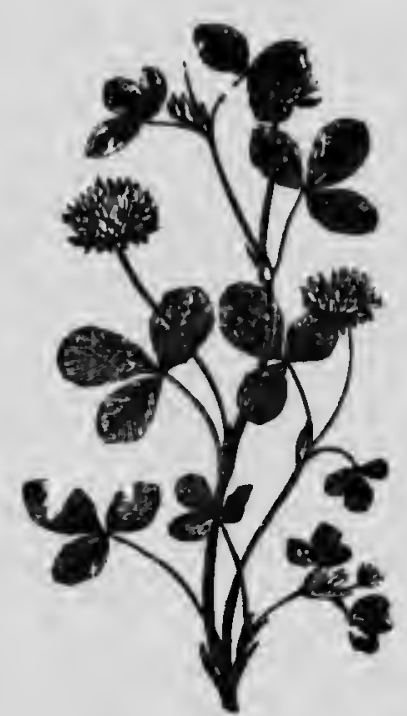

Fig. st. Tretie Alsike.

Ln cuiture la meilleure pour les abeilles t st sans contredit celle du Sai que nous ne cultirons mulhenreusement pas dans la Frovince de Québec On pent dire que si le rucher est entouré de champs le Sninfoin, on aura presque toujcurs une récolte de miel, wêne dans les années médiocres.

1--Si l'on veut se rendre compte en détail de la regertation mollifére do la coutré, on pent se servir d'un des onvrages appelés Flores pur trouver lo nom lles plantes. Daus la Vonuelle Flore de MM. (i. Ihommier et ile Jayeng

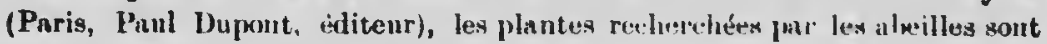
inliquées par un signe sperral. 
Les autres plantes fourrugèes à noter sont principalc. ment le 'Trètle llane quil, clıce nous est considéré comure le productenr dn meillanr uniel, le Trètt. incurnat, le Trètle hylricle, la llinette.

II fint eiter ensuite less champs de Colza ou même de Choux yni peuvent lonner une recolte importante. Le Siarmsin fournit à l'nutome un nectar abondunt arec lequel les abeil' es font un miel de mélivere yualité, mais recherché des putti-siers.
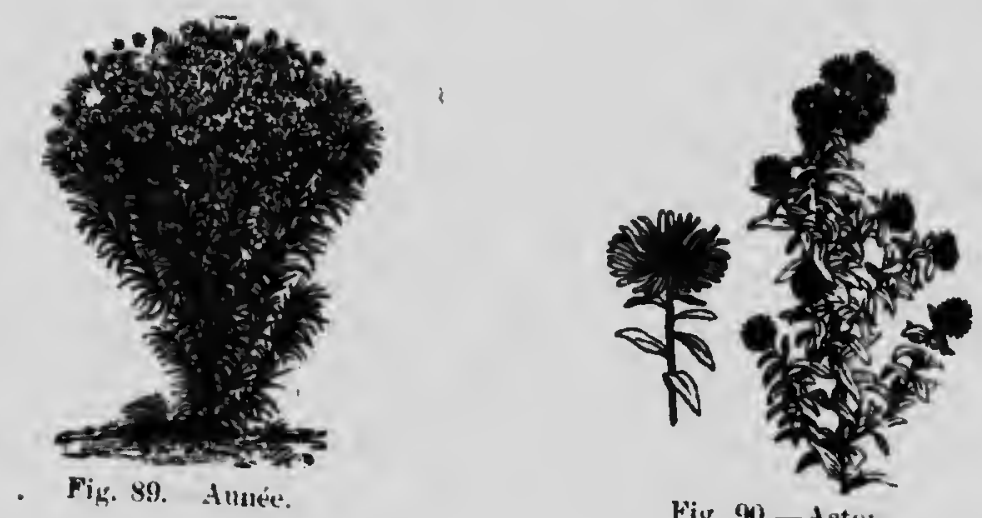

Fig. 90.-Aster.

Les chitmps de Blé, de Seigle, d'Orge, d'A roine, de Mnïs, de B-tterne, de Lin, ne pré-entent pour ainsi dire aucune ressource pour l'apiculture, si ce n'ent parfois lorsque ces champs um ct:ltivés contiennent certaines mauraises herbes mellifères qu'on peut trouver aussi sur le brord des cremins (Bluet, Pissculit, V'esce sauvage, Pied d'ulouette, etc).

Il ne faut pas oublier de signaler encore parmi les plantes cultivées pouvant produire du miel, les. Haricuts, les Puis, les Fives, les Oignons en fleurs.

Après ln moisson, les chanıps sont parf is envahis par duutres nnnvaizes herbes qui peuvent aussi rendre service aux alueilles : Epiaires, $G$ ıléopxis, Renonées, etc. 
Nous ne parlerons pas des plantes cultivées dans les jardins, car malgré leurs brillantes couleurs, elles sont, en général, peu ou pas visitées par les ubeilles. On peut copendant citer quelques pluntes de jarlin qui sunt mellifores : les Myosotis, les Corbeille-d'argent (Arubette des Alpes), les Aster, les Phucélia, etc.

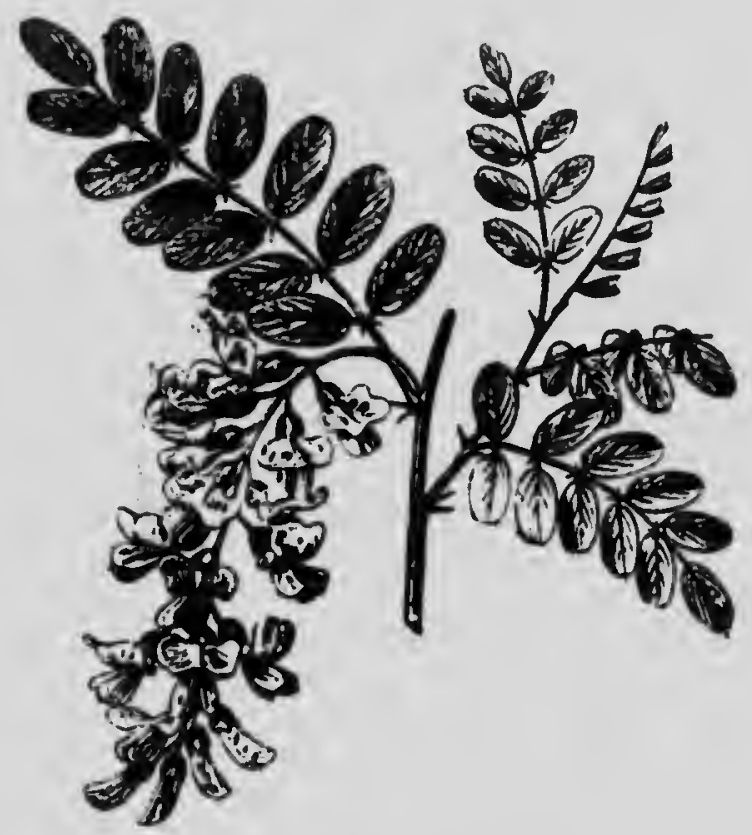

Fig. 91.--Aeravia (Heurs blanches).

Arbies mellifères. - Les arbres qui sont plantés sur le bord des rontes, daris les bois, lans les haies, ou même cultivés dans les chumps, dans les pares, peuvent parfois donner une récolte très importante. Au printemps, il faut citer surtout les Suules, les Erubles, los Cerisiers et les Pruniers. Quant aux Pommiers et aux Poiriers ils sont rarement mellifères.

Plus tard, ce sont les Fubiniers on Fuux-Acacias et res Tilleurs; le miel de ces arbres est souvent al,ondant. 



\title{
CHAPITRE QUATURZIE.IE
}

\author{
USATES IU MIET. - CONCIUSION
}

Les trarlitions de l'nutiquité ln plus reculée montrent que le miel a toujours été consiléré comme étant une nour. riture ngréable et suine. Pendnut les siècles l'houme n'a pas connu l'nutre snce.

Anjourd'hui que lu sève de la cnıne à sucre et de la betteruve, convertie en sucre, ist devenue une nécessité pour chaque fumille, vojons quelle plice le miel loit prendre duns notre léginc, non seulement comme nssuisornement semblable un sucre, mnis comme nourritule; boisson et médicument.

Le miel comme nourriture "st tıès lion pour la santé. On a remarqué que ceux qui mangent du miel a leurse pas trou vent à cette $h$ ıbitude swnté et longue vie.

"C"est une nourritnre offerte à l'houmc jar lu noture, toute préparée, extraite goutte à goutte de myrindes de flcurs, par un inoyen plus lélicat que celui qu'emploierait un luhoritoire humain."

(T.-di. Nrwmas, Money ax Food and Medirime.)

Comme nsisuisonnement le miel pent être employé de différeutcs fuçons: ians les honbons, il peut remplncer la glucose malsnine du commerce. Ises confiseurs quil'mploient voient leur clientèie augınenter.

Il remplace avuntageusement le beure sur la table surtuut pour les enfants. 
Le pain l'épice se vend en Fınnce, aux foires, en im. menscs quantiteq. Il peut être conservé inlléfiniment et bien des fenmes de cultivateurs en lunt les provisions pour plusieurs 1..uis.

\section{MIEL COMME MFIDICAMENT}

Le traitement de la chlorose par Ir -..iel est populuire dans le Danemark et le Hanovre. Les jeunes îlles paler des villes sout envoyées dans In caunpagne por:r prendre de l'exercice et manger lu miel. Les bo- ${ }^{-8}$ rénultats de ce traitenuent ont suggéré à I tehusar. la tlzéoric que c'ent l'insuff. snnce du sucre hépathique qui cause la chlorose, théorie qui expliquernit l'effet curntif au miel. (Jacoud duns la Revrie Internationile).

('n emploie une pate faite de miel et de farine pour enduire les furoncles, les meurtirisures, etc., après leur avoir fuit prendre un bain tiède d'enu salée de trois heures de durée. Cette pâte empreche le contuct do l'uir eb aide ainsi la nuérison.

Des boissons sucrées de mel guérissent le mal de gorge, In toux et elles peuvent nriêter le développement de la diphtérie. Un verre de vin oll de cidre, ou un bon grog, fortement sucrés de miel, ont été recomm andés par M. Hamet dans l'A piculteur, comme excellents pour arrêter la toux.

Les enfants d la mamelle qui souffrent de la constipation peuvent être fucilement guéris el leur nouant dans un petit carré de linge un peu de miel brosé avec du pain, qu'on leur donne \& sucer.

L'usage de miel aux repas a guéri les plus nonuvais cas d'hémorrhoïdes.

Suivant M. Woiblet, on peut guérir les verrues en se lavant les mains avee de l'eau miellée. Ayant entendu parler de ce moyen, il mit un emplatre de miel sur la main 


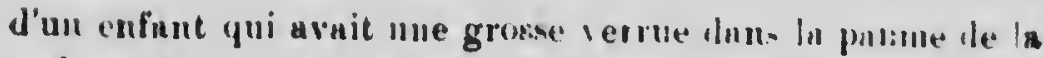

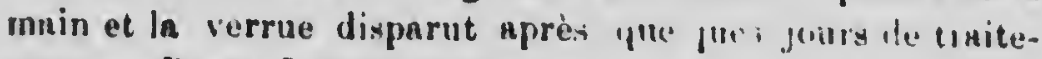
ment. (krene Inlermalimule.)

Nons nurions pu nous étemire flus longrement sur l'emploi du miel, mais mons mons fusé yuil sthait de

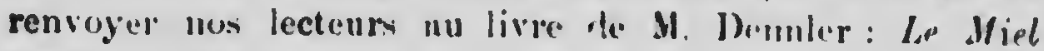

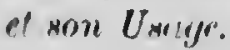

MIEI, EN HOISSON

Le uélunge d'ean et de miel, nprès formentution, a ro ciu le nom d'hydronerl. Cette liqueur étnit crmmue dess les temns les plus recules. Dnns les mys du nud, elle remplingnit le vin uvant l'invention de la bière. L'hydromel a cuses létrac. teurs et ses pmitisans. S. Benmuier écrionit, ell is $6:$ : "Il énivre plus faeilement que le vin; muis cette bois-on est stomacule et cordiale, en ce qu'elle est propre ì runimel les

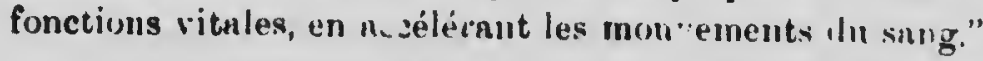

Les rocettes pour raire l'hyllomel, ninsi que les monntes nt on le paifume, varient nutıut que celles ilu pmin d'épice. Les uns le fout bonillir, les antres le font ì froil.

Voici la méthoile préconisée par II. de Iaryens.

Utili.sation des eaux de lar"ge dans la fabrication de l'hydrcmel - Après ln récolte, on jette dans un bnquet les opercules chargés le iniel qui cut été précédemment égouttés sur des tarnis; on y noule le l'crur, puis on les mélange hien uvec l'enu ufin d'en séparer tont l. miel. A mesure que in cire des opercules m vite al la wifnce, on en fait avec les mnins des boules qui seront ntilisées plus tard pour la fabrication dic la sire.

On joint ensuite ì ces taux, déjà chargees de miel, celles provenant du lavage de tous les instruments qui ont servi pendant l'extraction.

On doit utiliser les eaux de invage a la fabrication de thydromel le plus tôt possible, car si la température est 


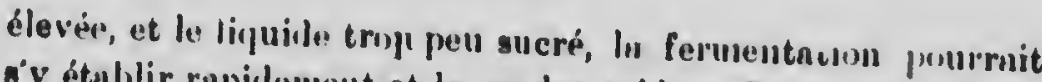
n'y étublir rapillement et le renil re ncide. Daus le cas sic on nurait ntteulu trop longtemps, ces eunx us servirout plus à faire l'iygliomel, car ce deruier pourruit tomoner un viunigue après In fermponfution.

Jorsquinn pissède ninsi des enux mielléen, onl y fait Hotter le glucomètre. Supposous pur exemple que l'iustru. inent maryne 5"; on levern nlars fnire disoomdre dans le liquicle unt suffismute qunntité de miol pour y uncuer le glucometre in murquer 17". Oll verse ce linguile dinus un tonnen., que l'on He remplit pus tout is finit in chuse de lu fernentation qui se produit; il fnut y njouter un Imélnuge de iniel et d'ean contenun la mêne proportion de uniel.

Pur une simple règle de trois, on trouve fueilement la quantité le miel et d'enu ajonter aux eaux miellées déja versées dlesis lo tunucnu et qui ne le rempliswent pas.

Si un n, pur "xemple, un tounenu de 100 pintes dans

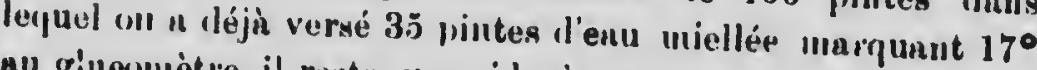
uu gineomètre, il reste 111 vide ì remplir de 64 pintes car on laisse toujours, comme on l'o vu, une pinte de vide environ.

Il est fucile de culculer la quautité de iniel et d'san d ajouter pour remplir le tonneau. fundru :

Si duus 101 pintes d'ean, il y 1 3is piutes de miel, il an

$$
10 i+64=2 \cdot \text { pintes, } f 0 .
$$

Il faut lonc fuire un unélnuge le $(54-22,40=41,60$ pintes d'en et rle 22, 40 pintes de miel que l'on devra ujouter nu tonneul.

Temps nécessaire a la fermentation. - In formen. tution est plus ou un rius rapide suivant que la tempéruture est plus onl moins ólevée. FII été on nul printeın|s, on loit placer las tounenux lehors, nu soleil; en hiver, alans me 
cave, un cellier, une cuisino, etc.; mnis jamais lons mus pidco oì il y $n$ du vinuigre.

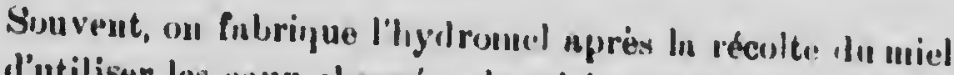
atin d'utiliser les euux chargíes de miel provennnt" Invage des operéules; inus ce cus, In frrmentution n lien lontencut

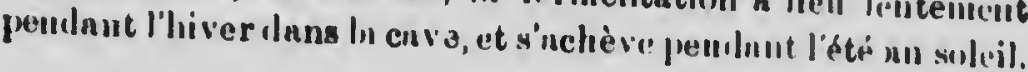

Par suite de In fermentation, le f: ide haisse 11 pren

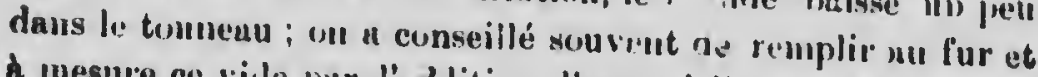
h mesure ce vill pur l'uddition d'enn miollev; celn n'n sucun
avanthge.

Lorsyuion lientend plus pétiller le lipuide, on renpplit

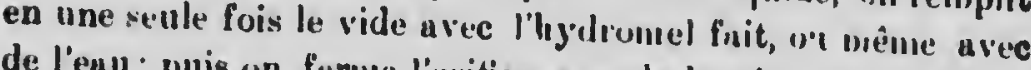
de l'enu; puis on ferme l'uritice avec !a bonde. On I'n plus

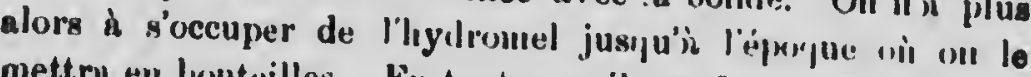
mettru en bonteilles. Sil tont chs, il ne funt jus le trins. vaser d'un tomıenu dous un hutre.

En général, quand ln fermentution est teroninée, c'est-u-

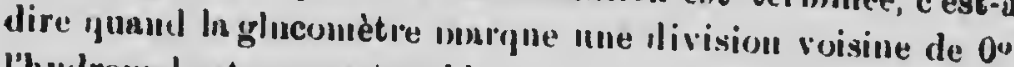
l'hydromel est encore tromble.

Entre l'époxplue ni l'hÿlromel a terminé sa fermentation et celle wit il rlevient cluir, il peut s'écouler mn temps trds long, six ıvis on mêıe plus l'un an; cela lepend de la nature des miels et l'nutres cuuses encore qu'il n'est pas actuellement possible de déterminer.

Le mieux ext d'uttenlıe qu'il s'écluircisst de lui-mêno, cur il n'ucquiert de qualité qu'en vieillissant. En général, l'hydromel s'éclnircit plus vite en hiver qu'en été.

Collage de l'bydromel et mise en bouteilles. - Dee tonneaux et de lour entretien. - On vient de voir que, lorsque la fermentation est terıninée, le meilleur est d'atten. dre que lt trouble disparaisse de lui-ınême. 
I'outefois, si duns certuines circonstunces, ce trouble semblait persister indéfiniment, on pourrait tent $r$ de huter In claritication par le collnge.

On colle l'hydromel avec des blanc d'uenf co:mue le vin. Apres le collage, on devra attendre, avant la mi te ell bou. teilles, que le liquide soit purfuitement clair.

Dans le cus où l'hydromel resterait troub'e rpiès le collage, c'est en général simplenient parce qu'on a co'lé trop tot; si colr se proiluit, on r'nurn qu'd recomın uncer plus tard.

La mise en bouteilled se frit comne pour le vin ; mnis comme l'ty 'romel travaille souvent encore, il sem priment de laisser les houteilles debout pendant un certnin temps Si l'hylromel est encors légèrement sucré, on pourra fuire de l'hylromel mousseux; on se servirn de bo'iteilles de chumpagne, les seules qui résistent à une forte pression, et après avoir consolidé les bouchons à l'aide de fil te fer. on pourra coucher ces bouteilles.

Vinaigre de miel. - On pent fuire très ficilenient avec d" miel et de l'eun un excollent vimigre dont l'origine est certaine, tt qu'on derrn employer de préférence an liquide furfoi- freluté on dungereux que l'on vend dins le commerce sous le nom de vinnigre.

Vivici le pricédé :

On remplit anx trois y ${ }^{\cdots}$ - ts un tonneau avec un mélange d'eau et de iniel contenant 10 p. 100 de miel. On ferme la bonile pur une tuile ou une pierre qui permet le passage de l'air, et on place le tout lans un endroit chaud et au soleil. Buit ou dix mois après, le vinaigre est boll dì consommer.

Il ne fuut jamais mettre ce tonneau dans une cave ou se trouve des torneaü de vin ou d'hydromel, car le ferınent 
ac étique pourrait s'y communiquer et les fuire touruer. II ne faudra jumais non plus se servir de tonneau ayant contenu le vinaigre pour $y$ mettro de l'hychonel ou du vin.

On pourrait abréger la fubricution de ce vinaigre en semust duns le tonneau, après la grande fermentation, ce qu'on appelle vuiguirement " une mère du vinaigre ".

A mesure qu'on soutire le vimigre du tonneau, on le remplace par de l'hydromel plus ou moins étendu d'eau ou de l'eau miellée dans la proportion de 10 pour 100 de miel. 


\section{CONILUSION}

Les instructions que nons avons données, pour la conduite des ruches ì culres moliles, s'udressant uux comniençants surtout, nous n'avons pas inentionné toutes les opérutions pratiquées par l''s apiculteurs expérimentés en vue de hâter le développement des colonies; nous arons au contraire cherché à mettre le débutant en garde contre les dangers que certnines d'entre elles présentent, lorsqu'elles sont tentées par lles mains novices.

On a pu voir yue nous exigeons, pour la culture les abeilles, une certuine dose de soin, de vigilance et l'observation. Nous ne nous soucions pus le faire de unuvaises recrues et ne sommes point fâché de contıibuer punr notre part à déraciner cette opinion trop généralement répanlue que les abeilles ne demandent pas de surveillance et qu'nvec elles, on peut récolter sans nvoir semé. Un rucher, à moins qu'il ne prenne l'importance qu'on donne à une spéciulité, ne demande certes pas beaucoup de temps, mais il lui faut quelques soins indispensubles, donnés à pripos par quelqu'un qui trouvé du plaisir a la chose.

A nıesure que le débntant acquerra de l'expérience, il trouvera de lui-uême les simplifications dont penvent être susceptibles certaines opérations, de même qu'il a/prendra petit à petit à apprécier l'un conp d'oil les conditions d'une ruchée et à se renılre compte pronipteinont le la cnuse les désordres ‘ui pellvent s'y produire. Devenu upicnlteur, il se convaincra que la coniluite de quelques ruches est ì ln portée même les personnes qui ont pêl le loisirs; qu’h̀ 
l'exception de la première visite lu printemps, du prélèvenent du miel et de la mise en hivernage, qui représentent ensemble quelyues heures cle iravinil, le reste des vpérations et les petites tommées de surveillance peurent se fuire en peu de minutes lans les moments perdus. 'Toutefois s'il accepte notre traite comme grnicle, qu'il veule bien, tmit qu'il sera dons sa période d'apprentinsage, ne pas éprigner la surveillance et suivre fidèlencent toutes les instructions et recommanelations, que nous arons, nutant que possible, accompagnétes de développements les explipunnt et les justiHant.

Le succès en npiculture dépend du développennent que les ruchées ont atteint uu moment vi la miellée se présente. Puur ubtenir un développement complet et opportun, il funt: de bonmes reines, de jeunes abeilles i l'uutumne, uu bon hivernnge qui prépare de bommes nourrices pour le printemps, d'abonduntes provisions au moment de l'élevagre du couvain et entin des ruches chaudes. silscejtibles d'être graduelleınent et consilérublement agranlies. Un rucher ne peut être en plein rapport que lorsque sun propriétuire possede une umple provision de rayons, et pour hâter l'arrivée de ce mument, il doit faire usage de feuilles gnufiées et lu mello-extracteur.

Le débutant fern bien de ne conmencer quinec peu de colonies, deux un trois au plus, et de ne pas se lécuurager si, dans. les pı enièı es aunées, ses housses de ruches n'arrivent pas à êtru intièıement remplies par les abeilles at le niel. Souvent les reines provenant de petites ruclıes vulguires ne cont jos si fécondes que celles qui seront élevées par sélection torsque les colonies ont pu se développer nurmulennent.

Nous désirons aussi mettre le lecteur en garde contre les dires de certains auteurs aflectant de professer qu'on peut faire de bonne apiculture arte n'inpuste quel outillage. C'est une bien facheuse notion à inculquer ì un délutant et 
le devoir de ceux qui veulent propnger la culture des abeilles est, au contraire, de mettre entre les mairs des novices les modèles les plus conformes unx principes généralement admis et les plus propres à leur épargner les fuusses manneuvres pt les insuccès.

Pour notre usage, nous préférons les ruches verticales, à pluncher et à plafond mobiles. Nous ne saurions recommander les ruches horiznntales qui ont bien leurs a vantnges, mais qui sont d'un mn niement.trop difficile pour l'hivernement.

En résumé, nos inéthodes et l'outillage dont nous con: seillons l'emploi ne nous sont point propres. Après avoir étudié consciencieusement, nous osons le dire, les procédés de culture des différentes cuntrées et recueilli les résultats de l'expérience, nous offrons simplement le fruit de nos études en recommandant ce qui nons paraît devoir le mieux réussir.

\section{E R R A T A}

Page 26. - Jisex. Figure 10. - Ovairen.

Page 128. - be ligne, Linez: Figure 56hi., page 129. 


\section{OUVRAGES A GONSULTER}

L'abeille et la Ruche.-DAHANT.

Conduite du rucher.-BERTRAND,

A. B. C.-d'A piculture, par E. Rocr', Mctina, Ohio.

Canudian Bee Journul.

Americun Bee Journul.

Cours complet d'apiculture.- LE LAYESS. 


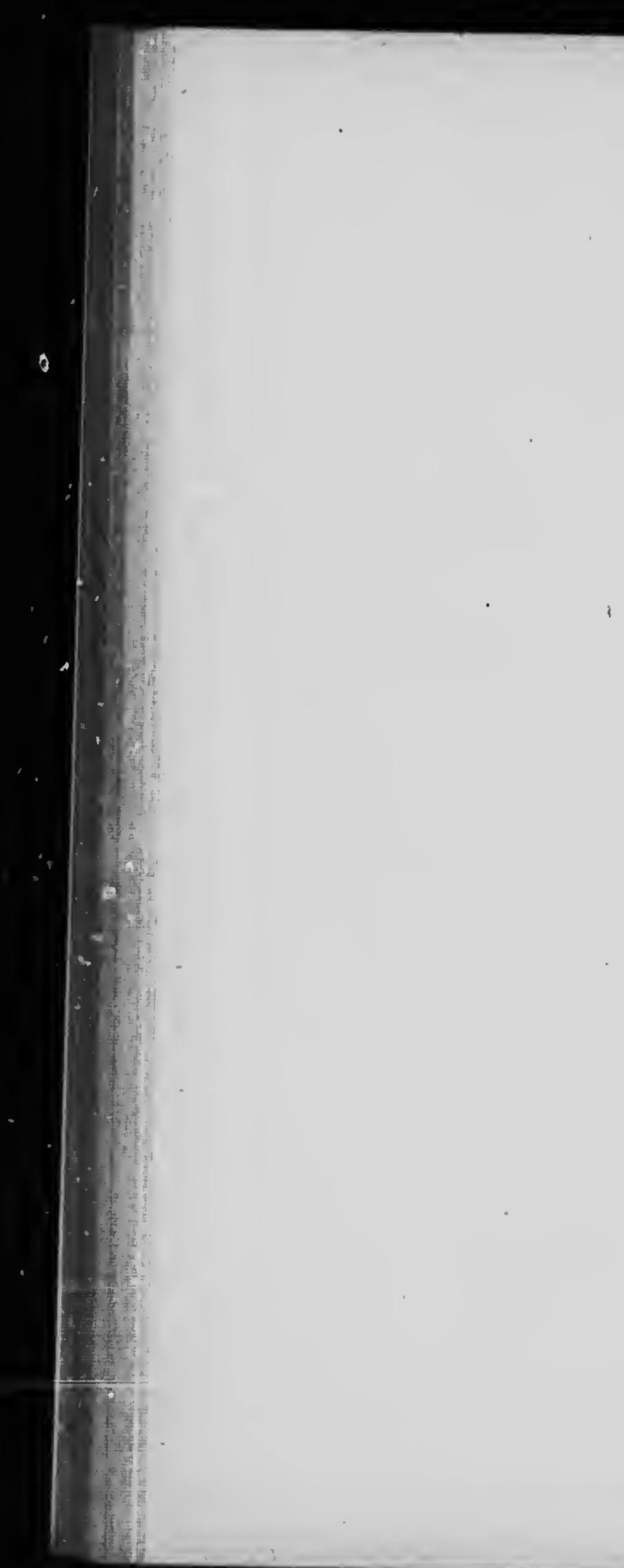




\section{TABLE DES MATIKRES}

Abellle. - In Page

"

o

Caraclères généranx............................. 10

" Durée de la vie............................... 16

Alimentution. - A ntomne............................ 166

Printempn ........................... 154

de reines...................................... 33

Aplon

Aplomb des rucher.................... . . .............. 62

Bingham.............................................. 141

Cudres.-Diversité ........................................ 115

Caisse d'expédition................................................ 132

Cave.-Hivernenrent................................ 167

Cellules. -Reine................................. 28

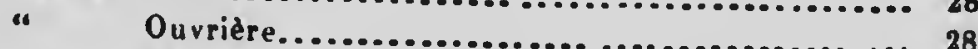

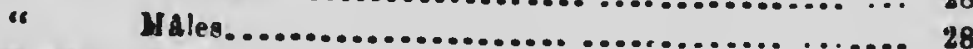

Chaleur (trop de)...... ........................... 103

" Hivernement............................... 170

Chapitean............. .......................... 88

Chasreabeiller.......................................... 130

Clre. Extraction.................................... 146

"r Ganffrée........... .............................. 147

« Cylindre pour la................................... 104

"6 Root............................................. 107

" Fils de routiell ..................................... 113

"s Iniluatrie........................................ 148

" . Machinea pour la.................................... 106

"r Couper la............................................ 114

a E-pèces...................................... T10 


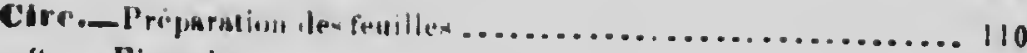

“ Fixer la................................... 112

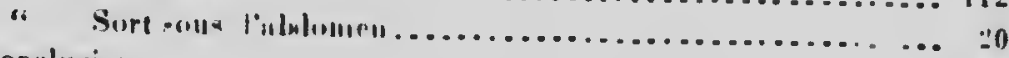

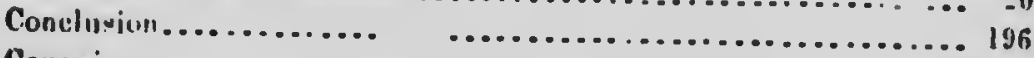

Courain..........................................10-14

Cuve n opelculan..................................... 142

Dentier les ruclses................................... $8^{\text {* }}$

Deplacemeni de ruchre. ...................................

Diarrhée....................................... 178

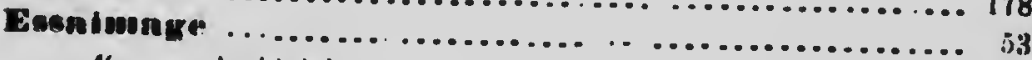

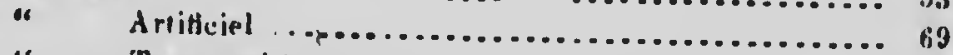

" Trnn-posilion................................ 70

" Coionien priférée........................... 70

، Wivi.ion, ............ 70

"، Vuturel, can

" Natirel, call<en....................... 54

" Prévention............................ $68^{\circ}$

"، Srinaire, Secunilaire ........................66-57

" Misi en place dem.... .................... 66

" Recolte................................ 59

Extracteur......................................... 110

Extrnetlon. - Opération............................. 137

« Untensilen.............................. 14

Farilie........................................... 100

Fecondation de In Reine................................... 28

Flore mellifere....................................... $\mid{ }_{1 \times 1}$

Gants v'apiculteur....................................... 9

Fambangh, ronlelie .............................. 113

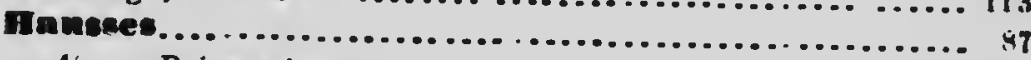

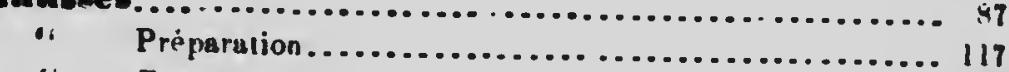

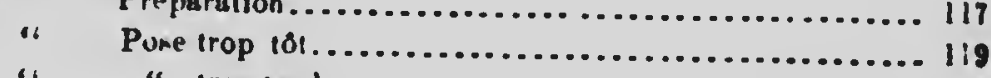

، "

, Secolide...................................... 120

. Miel d'automne................................. 121

" Virite el récolte.................................. 121

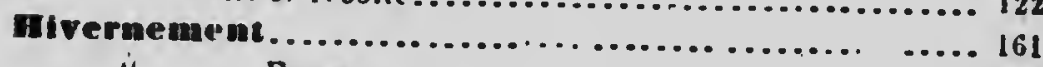

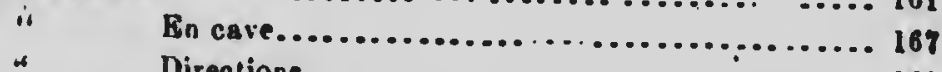

. Directions..................................161

" Temperalure.......................... 170

" En pheis air $\ldots \ldots \ldots \ldots \ldots \ldots \ldots \ldots \ldots \ldots \ldots \ldots \ldots \ldots . . \ldots 165$

" En ailos $\ldots \ldots \ldots \ldots \ldots \ldots \ldots \ldots \ldots \ldots \ldots \ldots \ldots \ldots \ldots$ it 
Mive rapaneut. - Syatdme hingham .................. 173

Hydromel.............................................. 191

In aecten eunemin den nbeilles.............................. 180

Larves l'abeil'ep.......................................... 10 14

Loque, Maladie .. ...................................... 176

Maladies des abeilles..................................... 176

Males ............................................ 10

- Fonction .......................................... 18

4 Mag-ncre................................. 18

Maniement les abeilles.................................... 97

Miel. - Bien prénenté . . .............................. 13.3

" Bulies..................................... 145

"1 En Loismons...................................... 191

" Mèdicament.................................... 190

" Vollrriture ...................................... 189

" En sections..................................... !24

" Extraction................................. 137

48 Extrail ..................................... 133

" U.t.nailes.......................................... 140

Nettornge ties ruches................................... b:

Nourriasement d'automne............................... 156

" le priutemp .............................. 154

Nourrisqeurs............................... 15ti, 167.158

Nonrriture................................. .... 185.159

Ouvières, fonctions................................... is

" pondenaeq.................... ............... 49

"6 Vieillesse................................... 18

Oraireg le la Reine....................................... 26

Paillagron............................................ 88

Parker............................................... 129

Peinture des ruches....................................... 9t

Planche le purtition.................................. $\times$ \%

Pillage........................................... 99-100

Piqures.............. ............................... 98

Plantes mellifèren..................................... 182

Platean............................................... 80

Ponte, Canses de In..................................... 14-154

Fropulin............................................. 20

Protiaionf........................................... 41

Rayone.............................................. 19 


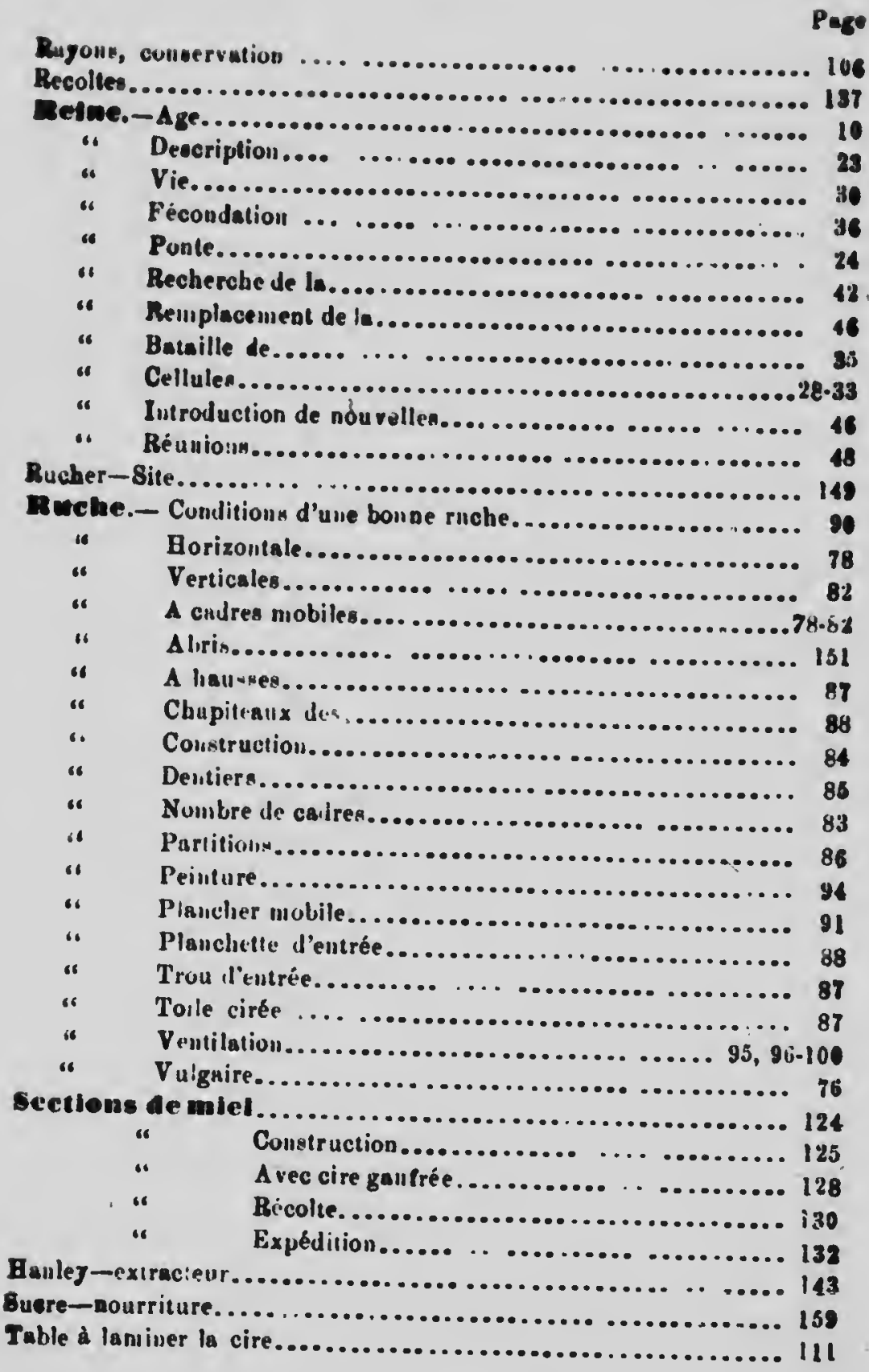


Teigne - ravager den............................. . 178

Toile curée........................................... 88

Tranapori des ubeillew............................. $46,167.169$

Unagen dil milel........................................ Isp

Utilite den uheilles pour l'agriculure...........................

Ventilenren ................................................. 100

Ventillntion ............................................. 9596

Vinaigre de rirel.......................................... 194

Vinlier...................................................95-123

Voilem itapiculteur........................................ 99

Vol unptial de la reille..................................... 37

Woiblet ........................................... t8 


\section{Ptablissement d’Apicultupe}

RUCHER MILLAFLORA

CHRRLESBOURG. - QUEBEC.

N VENTE A OET ETABLIS8EMENT

Abeilles,

Oire pure d'abeilles,

Miel en rayon,

suches a cadres mobiles,

Bestions a mie', Supports de sections,
Reine d'abeilles,

Miel de tride blanc,

Cire gauffréte.
Magasin a miel,

Cadres a rayon, Separateurs.
Enfumoirs,

Metal perforé.

Couteaux a désoperculer.
Nourrisseurs, Extracteurs,

Eperon Woiblet.
Oages a siveoles.

Bourdonnieres, Chasse abeilles,

Cages a reines,

Arrôte-pillage,

Cisailles.

Gants on ca utchoux,

Gants on tinsus croutchoute.

Voiles on tulle.

Verres a miel.

Bocaux a miel. Bcite a miel a section.

Seringues, Pompes a arrosage,

Thermomitres, Etc., Etc., Etc.

\section{UACQUES VERRET}

MARL HANID D'ARTICLES DAPICULTUIK:

PROPRIETAIRE DU

MUQAGUN DI GRAINME DE VERRET

OFARIUSBOURG, - QUEBEO. 


\section{R. F. HOISI'ERMAN APIOULFzon}

\section{HIRAN'TF(OKI), ON'IAIRIO. undili, llunar}

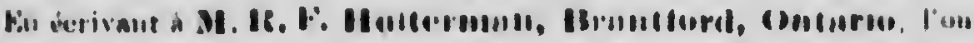

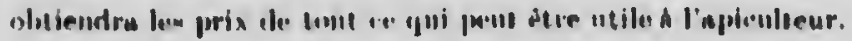

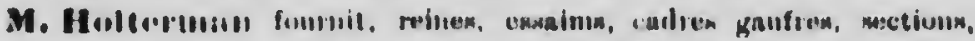

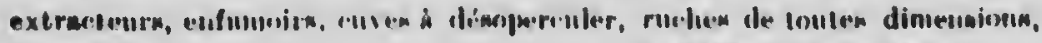
hulloece etr:., ele.

A DEs PRIX MODHRth.

SPECIALITE DE REIWES ET D'ESSAIMJ

6e.

ar lectout de prembire qualles to

\section{A. I. ROOT Co. \\ MEIINA, - O IIIO \\ wanklering.}

Ruches.

Heuseos.

Oedres.

Eectonn.

Oire sautroe.

Boltes d'expedition.
Extractours.

Relner-1tallennes.

Denetnus,

Boltes et veseu pour le milel. Dec., Erec

ECRIRE POUR CATALOQUE.

GOOLD, SHAPLEY \& MUIR CO. BRANTFORI, CANIIA.

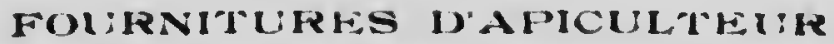

Ruches complètes de toutes dimensions. Cire gautréo pour calres et sections. - Extrncteurs. - Cuves d désoperculer. - Verres et boites ponr le micl.-Contiun à désopurculer. Reines. - Essuims. - Nonriswenrs, etc etc. 


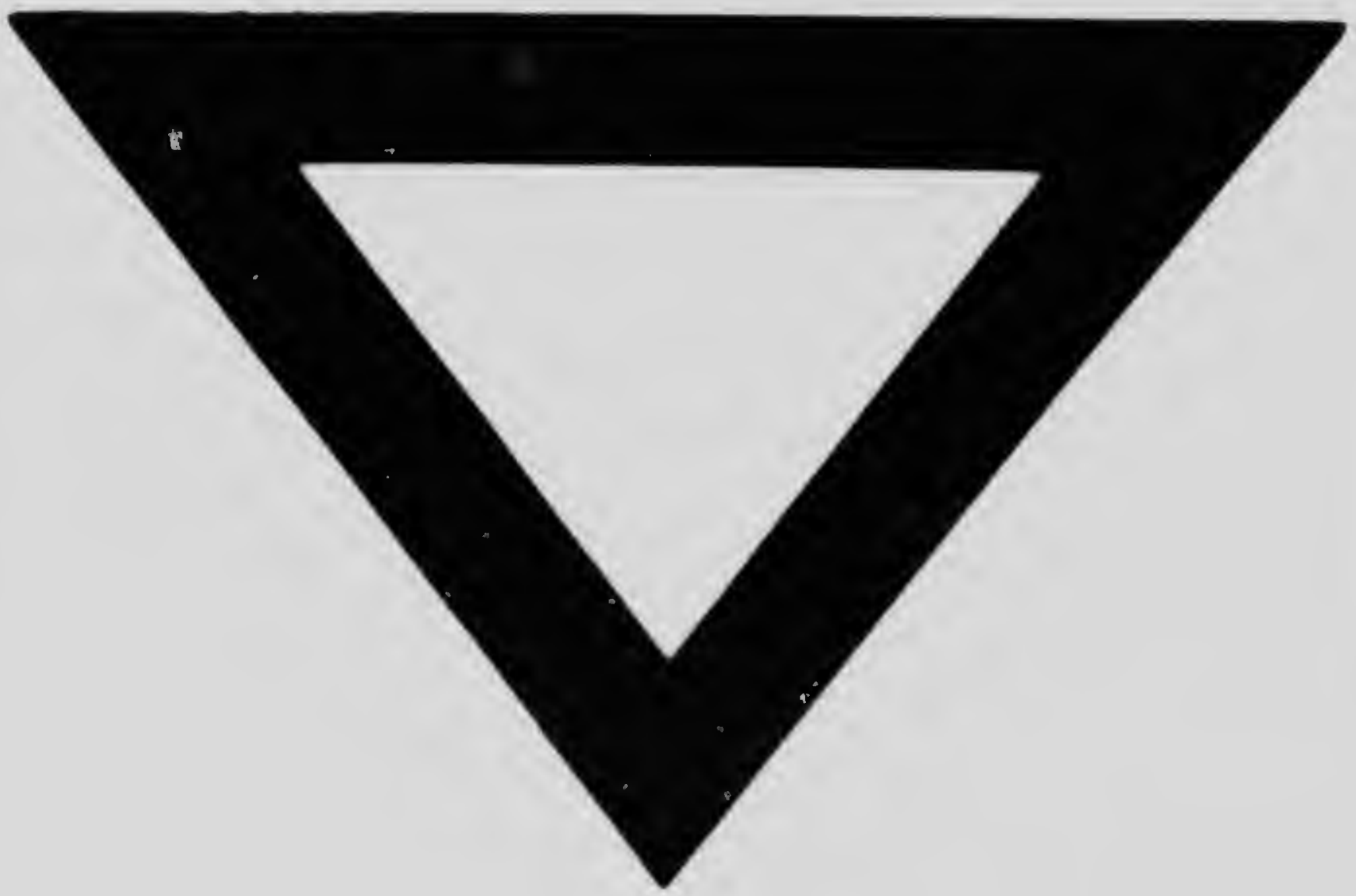

